\title{
Untersuchung der Stromverdrängung im Ständer hoch ausgenutzter elektrischer Maschinen
}

\author{
Von der Fakultät für Elektrotechnik und Informatik \\ der Gottfried Wilhelm Leibniz Universität Hannover \\ zur Erlangung des akademischen Grades \\ Doktor-Ingenieur \\ (abgekürzt: Dr.-Ing.) \\ genehmigte Dissertation
}

von

Dipl.-Wirtsch.-Ing., M.Sc.

Christoph Junginger

geboren am 27.08.1986

in Cham 
1. Referent Prof. Dr.-Ing. Bernd Ponick

2. Referent Prof. Dr.-Ing. Andreas Möckel

3. Referent Prof. Dr.-Ing. Axel Mertens

Tag der Promotion 11.10.2016*)

*) Datum der mündlichen Doktorprüfung 


\section{Kurzfassung}

Die vorliegende Arbeit zeigt, dass das etablierte, über hundert Jahre alte, analytische Berechnungsverfahren zur Bestimmung der Stromverdrängung mit seiner Annahme eines reinen Nutquerfeldes für hoch ausgenutzte elektrische Maschinen mit flachen Massivleitern nicht ausreicht.

Die Arbeit legt dar, dass es durch zusätzliche Effekte zu einer signifikanten Beeinflussung der resultierenden Stromverdrängung kommen kann. Mit der magnetischen Sättigung, der nebeneinanderliegenden Anordnung von zwei zu verschiedenen Strängen gehörenden Spulenseiten in einer Nut und der Wechselwirkung mit dem Luftspaltfeld werden drei wesentliche Ursachen für zusätzliche Stromverdrängungseffekte identifiziert und behandelt, welche mit den im Stand der Technik beschriebenen Methoden keine ausreichende Berücksichtigung finden. Für die magnetische Sättigung und die nebeneinanderliegende Anordnung von zwei zu verschiedenen Strängen gehörenden Spulenseiten in der Nut werden die Zusammenhänge in analytischer Form dargestellt.

In der Arbeit werden in weiten Teilen Untersuchungen mittels Finite-ElementeRechnungen durchgeführt, um zusätzliche Stromverdrängungsursachen zu bestimmen. Dazu wird zunächst eine geeignete Form für die Auswertung der FiniteElemente-Simulationen entwickelt, da es bisher noch keine etablierte Methode für diese Anwendung gibt. Aus den Betrachtungen werden nach Möglichkeit analytische Ansätze zur Bestimmung der Stromverdrängung abgeleitet. Die analytische Berechnung ermöglicht es, bereits im frühen Auslegungsprozess die tatsächlich auftretenden Wechselstromverluste zu bestimmen. Die vorgestellten Methoden ergänzen das gängige analytische Verfahren. Die entwickelten Methoden werden mit Hilfe von numerischen Vergleichsrechnungen und Messungen am Prüfstand auf Plausibilität geprüft.

\section{Schlüsselwörter}

Stromverdrängung, Wirbelstromeffekt, Widerstandserhöhung 


\section{Abstract}

The present thesis shows that the established analytical calculation method for the determination of the current displacement is not adequate for highly utilized electrical machines with flat solid conductors. It is more than one hundred years old and assumes a pure cross magnetic field in the groove.

The thesis states that additional effects can cause a significant influence on the resulting current displacement. Three essential causes for additional current displacement effects are identified: the magnetic saturation, the side-by-side arrangement of two coil sides in the groove, which belong to different phases and the interaction with the magnetic field of the air gap. Those causes are taken into consideration in the presented calculations in contrast to the state of the art techniques. The correlations are presented in an analytical way for the magnetic saturation and the side-by-side arrangement of two coil sides in the groove, which belong to different phases.

In major sections of this thesis, interpretations of finite element analysis are conducted to determine additional causes for current displacement. Therefore, an appropriate way for an evaluation of the finite element analysis is developed. There has been no technique for this application so far. Analytical approaches for the determination of the current displacement are derived as far as possible from the observations. The analytical calculation enables the determination of the AC losses that are actual appearing at an early stage of the design process. The introduced methods complement the common analytical procedure. The developed methods are checked for plausibility by numerical comparative calculations and test stand measurements.

\section{Keywords}

Current displacement, Eddy effect, AC resistance 


\section{Vorwort}

Die Arbeit in der Entwicklung E-Antriebe in Kassel hat mir viel Freude bereitet. Ich konnte dort in einer spannenden Phase viel von meinen aufgeschlossenen und freundlichen Kollegen lernen.

Besonderen Dank schulde ich Prof. Dr. Bernd Ponick, der sich bereit erklärt hat, mein Promotionsvorhaben zu betreuen. Er trug durch zahlreiche fachliche Diskussionen zum Gelingen dieser Arbeit bei, gab mir wichtige Hinweise zum wissenschaftlichen Arbeiten und ermöglichte mir diese für mich wichtige Erfahrung.

Dr. Gerd Stöhr hat mich während meines Promotionsvorhabens hervorragend betreut und mir den notwendigen Freiraum für eine erfolgreiche Umsetzung eingeräumt. Für die Lösung auftretender Probleme hat er schnell und unkompliziert gesorgt. Darüber hinaus hatte ich durch ihn die Gelegenheit, Teile der in dieser Arbeit vorgestellten Ergebnisse auf internationalen Konferenzen vorzustellen und zu diskutieren. Daran durfte ich wachsen.

Herrn Prof. Dr.-Ing. Andreas Möckel danke ich für das der Arbeit entgegengebrachte Interesse und die Übernahme des Koreferats. Des Weiteren danke ich Prof. Dr.-Ing. Axel Mertens für die Übernahme des Vorsitzes der Prüfungskommission.

Zuletzt bedanke ich mich noch bei meiner gesamten Familie, die mich immer unterstützt. Auch bei meinem Promotionsvorhaben stand sie mir mit Rat und Tat zur Seite und hat mir den Rücken frei gehalten.

Allen Genannten und auch allen anderen Kollegen, mit denen ich während meines Promotionsvorhabens zu tun hatte, danke ich ganz herzlich für die vielen wertvollen Begegnungen und Einblicke sowie für die Unterstützung, die sie mir gewährt haben. 



\section{Inhaltsverzeichnis}

Formelzeichenverzeichnis ......................................................................... IX

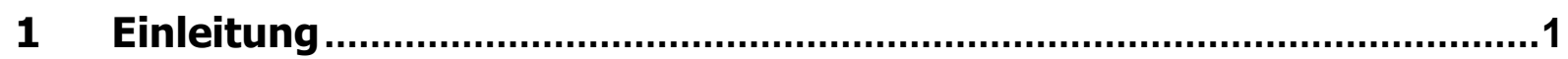

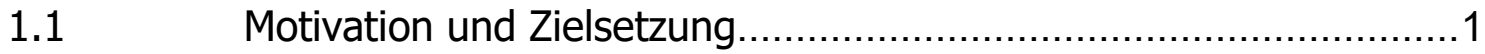

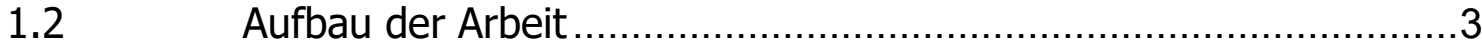

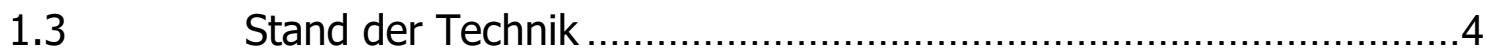

1.3.1 Chronologische Darstellung ....................................................

1.3.2 Sachbezogene Darstellung ………………............................11

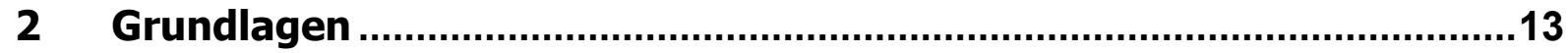

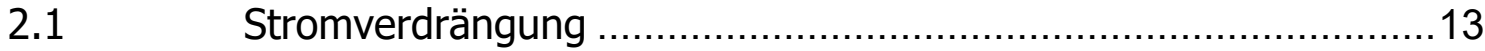

2.1.1 Verhältnisse ohne Stromverdrängung ...................................13

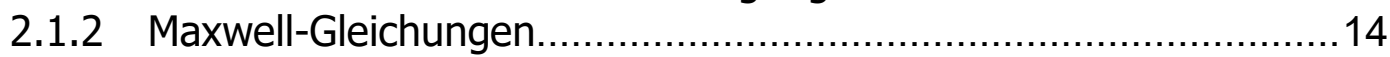

2.1.3 Eigenschaften des magnetischen Kreises ..................................16

2.1.4 Analytische Berechnung .......................................................18

2.1.5 Begriffserklärungen und -abgrenzungen zur Stromverdrängung...20

$2.2 \quad$ Permanenterregte Synchronmaschinen.....................................21

2.2.1 Einsatz als Traktionsantrieb in automobilen Hybridantrieben ........21

2.2.2 Verlustbetrachtung .............................................................23

2.2.3 Einflüsse auf den Polradwinkel .............................................23

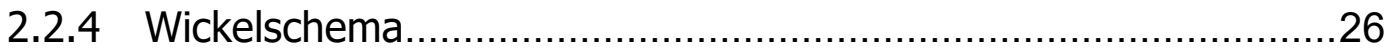

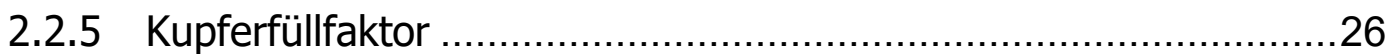

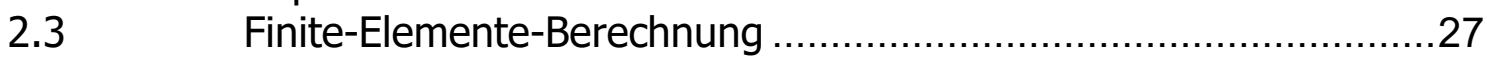

3 Vergleich des Widerstandserhöhungsfaktors.......................................29

3.1 Für den Vergleich verwendete E-Maschine.................................29

3.2 Analytische Bestimmung ......................................................

3.3 Bestimmung mittels Finite-Elemente-Methode .............................33

3.4 Messungen auf dem Prüfstand ...................................................35

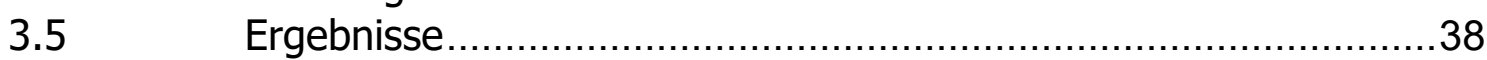

3.6 Einfluss des Permanentmagnetfelds ..................................... 41

4 Voruntersuchung zur Abweichung des analytischen Verfahrens .........43

$4.1 \quad$ Vorüberlegungen ............................................................... 43

4.2 Untersuchungsmethode und Finite-Elemente-Modell ..................45

$4.3 \quad$ Durchgeführte Voruntersuchungen .......................................47

4.3.1 Betrachtung des Nutstreufelds ...........................................48

4.3.2 Abgleich des Widerstandserhöhungsfaktors ...............................49

4.3.3 Einfluss der Nuttiefe auf das Nutstreufeld..................................51

4.3.4 Einfluss der Nuttiefe auf den Widerstanderhöhungsfaktor ............53

4.3.5 Einfluss der Nuthöhe und -breite auf das Nutfeld .......................54

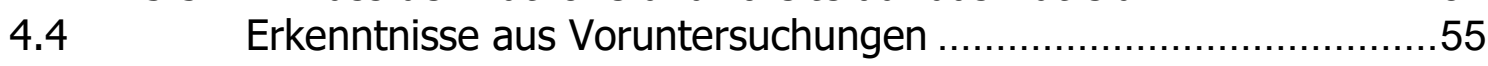

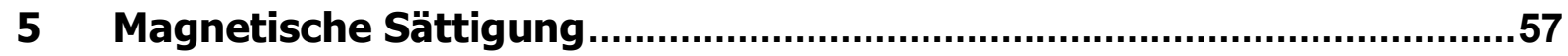

$5.1 \quad$ Grundlegende Zusammenhänge..............................................57

5.1.1 Verminderung der Querfeldkomponente ..................................58 
5.1.2 Zusätzliche Längsfeldkomponente durch Entlastung ....................58

5.2 Untersuchung anhand von Finite-Elemente-Rechnungen..............61

5.2.1 Modifikation des Grundmodells ..............................................61

5.2.2 Mit Finite-Elemente-Rechnungen durchgeführte Untersuchungen 62

5.2.3 E-Maschine mit gegossenen Spulen ......................................72

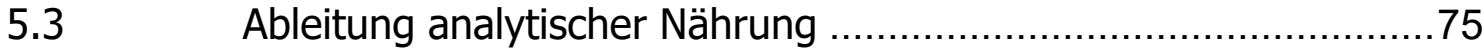

5.3.1 Verminderung der Querfeldkomponente ................................75

5.3.2 Zahnentlastungsfeld - Entstehen einer Längsfeldkomponente .....78

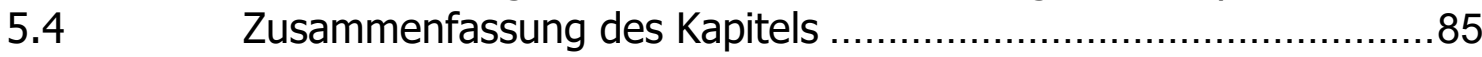

6 Phasenverschobene Spulenströme in einer Nut ...................................87

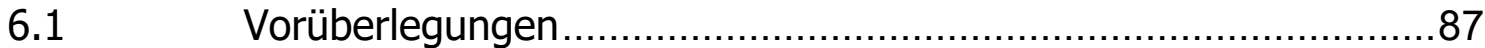

6.2 Betrachtung mittels Finite-Elemente-Rechnungen .....................90

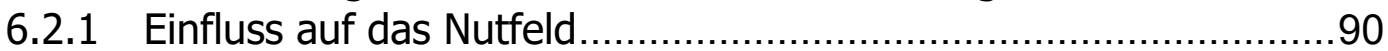

6.2.2 Einfluss auf die Stromverdrängung .......................................... 91

6.2.3 E-Maschine mit gegossenen Spulen ......................................95

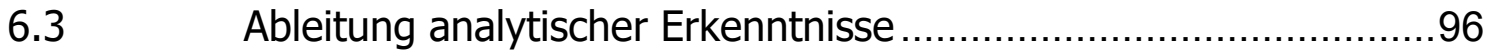

7 Wechselwirkung mit dem Luftspaltfeld.............................................101

7.1 Einfluss des Feldschwächbereichs und der Schrägung ................101

7.2 Einfluss des Konstantflussbereichs und der Schrägung ..............106

7.3 Drehmomentbildung und Zusammenfassung ...........................112

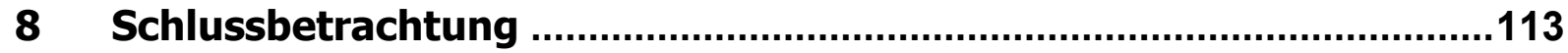

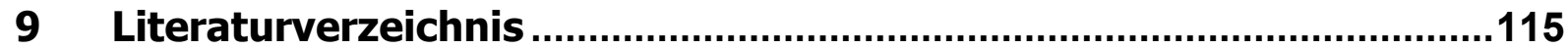

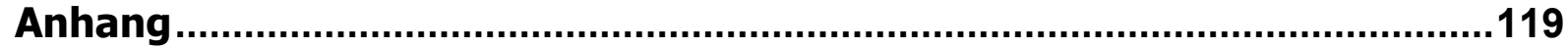

Anhang A: Widerstandsbestimmung gegossener Spulen.............................119

Anhang B: Messunsicherheit der $k_{r}$ Faktor-Bestimmung .............................120

Anhang C: Bestimmung der Magnetverluste mittels 3D-FEM .......................125

Anhang D: Ermittlung der Reib- und Eisenverluste ...................................127

Anhang E: Einfluss des Nuthöhe-zu-Nutbreite-Verhältnisses auf das Nutfeld..128 


\section{Formelzeichenverzeichnis}

\section{Lateinische Buchstaben}

$A \quad$ Fläche, allgemein

$A_{\text {/ }} \quad$ Querschnittsfläche der Leiter

$A_{n} \quad$ Querschnittsfläche der Nut

$a_{\overline{A B} q f}$ Verminderungsfaktor für die Stromverdrängung durch das Querfeld bei zwei nebeneinanderliegenden Spulen in einer Nut

$a_{\triangle A B l f}$ Verminderungsfaktor für die Stromverdrängung durch das Längsfeld bei zwei nebeneinanderliegenden Spulen in einer Nut

$a_{q f} \quad$ Verminderungsfaktor für die Stromverdrängung durch das Querfeld bei magnetischer Sättigung des Eisenkreises

alf Verminderungsfaktor für die Stromverdrängung durch das Längsfeld bei magnetischer Sättigung des Eisenkreises

$B$ magnetische Flussdichte

$B_{r} \quad$ Remanenzflussdichte

$b_{\text {I }} \quad$ Breite der Leiter

$b_{n} \quad$ Breite der Nut

$E$ elektrische Feldstärke

$f \quad$ Frequenz

$H$ magnetische Feldstärke

h, Höhe der Leiter

$h_{n} \quad$ Höhe der Nut

I,i elektrischer Strom

$\hat{\imath}_{\overline{A B}} \quad$ Amplitude der Spulenseitenstromsumme bei zwei zu verschiedenen Strängen gehörenden Spulenseiten in einer Nut

$\hat{\imath}_{\triangle A B} \quad$ Amplitude der Spulenseitenstromdifferenz bei zwei zu verschiedenen Strängen gehörenden Spulenseiten in einer Nut

$J$ elektrische Stromdichte

$J_{\text {mag }}$ magnetische Polarisation

$k_{\text {geom }}$ Verhältnis der Nuthöhe zur Nutbreite

$k_{r} \quad$ Widerstandsverhältnis zur Berücksichtigung der Stromverdrängung

$k_{\text {rslf }} \quad$ Widerstandsverhältnis zur Berücksichtigung der Stromverdrängung durch das Längsfeld

$k_{\text {rsqf }} \quad$ Widerstandsverhältnis zur Berücksichtigung der Stromverdrängung durch das Querfeld

$L \quad$ Induktivität

// Leiterlänge

$M \quad$ Magnetisierung

$N \quad$ Anzahl der Nuten

$n \quad$ Drehzahl

$P \quad$ Leistung

$P_{c u} \quad$ Verlustleistung in elektrischen Leitern

$P_{e l} \quad$ elektrische Leistung 
$P_{F e} \quad$ Verlustleistung im Blechpaket

$P_{\text {mag }}$ Verlustleistung in Permanentmagneten

$P_{\text {mech }}$ mechanische Leistung

$P_{\text {reib }} \quad$ Verlustleistung durch Reibung

$P_{V} \quad$ Verlustleistung

$p \quad$ Polpaarzahl

$R \quad$ elektrischer Widerstand

$R_{m} \quad$ magnetischer Widerstand

$S \quad$ Poynting-Vektor

$T$ Drehmoment

$t_{T} \quad$ Zeitintervall

$U$ elektrische Spannung

$U_{p} \quad$ Polradspannung

$U_{S} \quad$ elektrische Statorspannung

$V_{0} \quad$ magnetische Umlaufspannung

$v \quad$ Anzahl betroffener Nuten

$X \quad$ Reaktanz

$X_{d} \quad$ resultierende Reaktanz der d-Achse

$X_{q} \quad$ resultierende Reaktanz der q-Achse

$z_{n} \quad$ Anzahl der elektrischen Leiter je Nut

\section{Griechische Buchstaben}

$a \quad$ Reduktionsfaktor zur Ermittlung der reduzierten Leiterhöhe massiver Leiter

$a_{\vartheta 0} \quad$ linearer Temperaturkoeffizient

$\beta \quad$ reduzierte Leiterhöhe massiver Leiter

$V \quad$ Winkel zwischen der Stator- und der Polradspannung

$\zeta \quad$ Winkel zwischen zwei Wirbelströmungen

$\Theta \quad$ Durchflutung

$\theta \quad$ Phasenverschiebung der Spulenseitenströme

$\vartheta \quad$ Temperatur

vo Ausgangstemperatur

$\kappa \quad$ elektrische Leitfähigkeit

$\mu \quad$ resultierende Permeabilität

$\mu_{0} \quad$ Vakuumpermeabilität

$\mu_{r} \quad$ relative Permeabilität

$\rho \quad$ spezifischer elektrischer Widerstand

$\phi \quad$ magnetischer Fluss

$\varphi(\beta)$ Hilfsfunktion zur Berechnung des Widerstandserhöhungsfaktors

$\varphi_{c u} \quad$ Kupferfüllfaktor

$\psi(\beta)$ Hilfsfunktion zur Berechnung des Widerstandserhöhungsfaktors

$\psi_{P M} \quad$ resultierender Verkettungsfluss des Permanentmagnetfelds

$\omega \quad$ elektrische Kreisfrequenz 


\section{Indizes}

2 Zwei zu verschiedenen Strängen gehörende Spulenseiten in der Nut betreffend $\mathrm{cu}$ Kupfer

d d-Achse

Fe Eisen

g Gleichstrom

L längs der Nut

If das Längsfeld betreffend

n Nut

$\mathrm{N} \quad$ allgemeine Bezifferung

$P \quad$ das Hauptfeld betreffend

$\mathrm{p} \quad$ allgemeine Bezifferung

pp Phasenpaarung

Q quer der Nut

q q-Achse

qf das Querfeld betreffend

s die Sättigung des magnetischen Kreises betreffend

$\mathrm{u}$ unten liegend

$v \quad$ Verlust

w Wechselstrom

z Zahn

$0 \quad$ Basis 


\section{Formelzeichenverzeichnis}




\section{Einleitung}

Die Einleitung gliedert sich in drei Abschnitte. Im ersten werden die Beweggründe für die Beschäftigung mit dem Thema dargelegt. Der zweite Abschnitt gibt einen Überblick zu Vorgehensweise und Aufbau dieser Arbeit. Der dritte Abschnitt referiert erst chronologisch und dann sachbezogen den gegenwärtigen Forschungsstand.

\subsection{Motivation und Zielsetzung}

Die ersten Erkenntnisse zur Abschätzung der durch Stromverdrängung in den Spulen des Stators zusätzlich zu den Kupferverlusten bei homogen verteiltem Strom entstehenden Verluste liegen über einhundert Jahre zurück. Im Jahr 1905 hat Field [11] erstmals die Stromverdrängung in einem Leiter beschrieben, der an drei Seiten von ferromagnetischem Material umgeben ist. Weiterführende theoretische Betrachtungen wurden in den folgenden Jahren von Emde [8] [9] und weiteren Autoren publiziert. Frühe Ansätze zur experimentellen Überprüfung erarbeitete Richter [45]. Mit Hilfe dieser Erkenntnisse wurde es möglich, die durch Stromverdrängung zusätzlich entstehenden Verluste im Auslegungsprozess einer elektrischen Maschine ausreichend genau vorauszuberechnen und gegebenenfalls durch Anpassung der Leitergeometrie zu verringern. Allerdings beruht diese Bestimmung auf Annahmen, die zu einer Zeit getroffen wurden, als die Anforderungen an elektrische Maschinen sich teilweise deutlich von den heutigen unterschieden.

Seit den Anfängen der Stromverdrängungsbetrachtung haben sich die Ansprüche an elektrische Maschinen in verschiedene Richtungen weiter entwickelt. Nicht zuletzt die Verwendung als Traktionsantrieb in automobilen Hybridfahrzeugen erhöht bestehende und stellt zum Teil neue Anforderungen. Die Kernanforderungen an Traktionsantriebe in Kraftfahrzeugen sind ein großes Drehmoment und ein hoher Wirkungsgrad. Diese sollen in einem kleinen Bauraum und gegebenenfalls bei nicht frei wählbarer Drehzahl realisiert werden. Dafür kommen häufig hochpolige, umrichterbetriebene Synchronmaschinen mit eingebetteten Permanentmagneten zur Nutzung des Reluktanzmoments (IPSMs) zum Einsatz, die sowohl im Konstantflussals auch im Feldschwächbereich betrieben werden [25] [57] [53].

In realen Anordnungen bildet sich in den Nuten ein kompliziertes magnetisches Wirbelfeld, dessen Richtung, Stärke und zeitlicher Verlauf von einer Vielzahl von Parametern abhängt. Dieses lässt sich, aufgrund der komplexen Geometrie, analytisch nicht allgemeingültig berechnen. Eine analytische Berechnung setzt immer ein vereinfachtes Modell voraus [34] [50]. Bei der von Field vorgenommenen analytischen Betrachtung wurde die Annahme eines reinen Nutquerfelds getroffen, die für damalige Maschinendesigns hinreichend war und auch heute noch für zahlreiche elektrische Maschinen ausreichend genaue Vorhersagen ermöglicht. Die vorliegende Arbeit zeigt auf, dass die ausschließliche Betrachtung eines reinen Nutquerfelds für die im Absatz zuvor beschriebenen besonders drehmomentdichten Maschinen nicht mehr ausreichend ist - insbesondere bei breiten, flachen Leiterquerschnitten. Ziel dieser Arbeit ist es, den bisher gängigen analytischen Ansatz zur Bestimmung der Stromverdrängung durch eine zusätzliche Berücksichtigung der sogenannten Längsfeldkomponente zu ergänzen. Neben dem schon bekannten, in die Nut eindringenden Luftspaltfeld 
[50] [15] werden dazu weitere Faktoren untersucht, durch die die Längsfeldkomponente einen signifikanten Einfluss auf die Stromverdrängung bekommt. Besonderes Augenmerk wird hierbei auf drei Aspekte gelegt:

1. Magnetische Sättigung des Eisenkreises

Bei hohen Drehmomentanforderungen und begrenztem Bauraum sind Maschinen oftmals in ihrem Magnetkreis sehr viel höher gesättigt, als dies früher der Fall war. In der Literatur [6] [27] [15] [38] finden sich bereits Beiträge zur Wirkung der magnetischen Sättigung auf die Stromverdrängung. Eine tiefergehende Klärung der Zusammenhänge ist jedoch wünschenswert.

2. Nebeneinanderliegende Spulenseiten in einer Nut

Anordnungen dieser Art gibt es vor allem bei Zahnspulenwicklungen. Zahnspulen sind für Fahrzeuganwendungen aufgrund der kleinen Wickelköpfe eine interessante Option, um eine hohe Drehmomentanforderung aus kleinem Bauraum zu erfüllen. Auch zum Einfluss von zwei nebeneinanderliegenden Spulenseiten in einer Nut auf die Stromverdrängung existieren bereits grundlegende Betrachtungen [32] [10] [16], die im Rahmen der vorliegenden Arbeit vertieft werden.

3. Wechselwirkung mit dem Luftspaltfeld

Diese hat besonders durch den Umrichterbetrieb ebenfalls nicht vernachlässigbare Auswirkungen auf die Stromverdrängung. Sie wird vom Konstantfluss- und Feldschwächbereich, der Nutzung des Reluktanzmoments und der Staffelung des Rotors oder Stators beeinflusst. Auch zu diesem Themenkomplex finden sich in der Literatur Erkenntnisse [6] [11] [15] [36] [38] [46] [50]. Diese sollen hier um weitere Aspekte ergänzt werden.

Die genannten Gesichtspunkte werden mittels Finite-Elemente-Rechnungen untersucht. Das dabei gewählte Vorgehen ist in dieser Form neu und kann auch zur Untersuchung weiterer Aspekte der Stromverdrängung genutzt werden. Die Vorgehensweise macht den Zusammenhang zwischen magnetischem Feldverlauf in der Nut und resultierender Stromverdrängung sichtbar. Mit Hilfe der aus den Finite-ElementeBerechnungen gewonnenen Erkenntnisse werden Ansätze abgeleitet, wie die Stromverdrängung durch die Längsfeldkomponente auch auf analytischem Wege berücksichtigt werden kann. Dadurch wird eine ausreichend genaue Verlustberechnung der auftretenden Stromverdrängung im frühen Auslegungsprozess möglich. Außerdem führt das Vorgehen zu einem besseren Verständnis des Zusammenspiels von Nutquer- und Nutlängsfeld. Da Faktoren, die das eine schwächen, oftmals eine Stärkung des anderen zur Folge haben, ist ein tieferes Verständnis dieser Zusammenhänge für eine optimale Wicklungsauslegung hilfreich. 


\subsection{Aufbau der Arbeit}

Die Arbeit ist gemäß Abbildung 1-1 aufgebaut. In Abschnitt 1.3 wird ein Überblick über den bisherigen Stand des Wissens zur Stromverdrängung in elektrischen Maschinen gegeben.

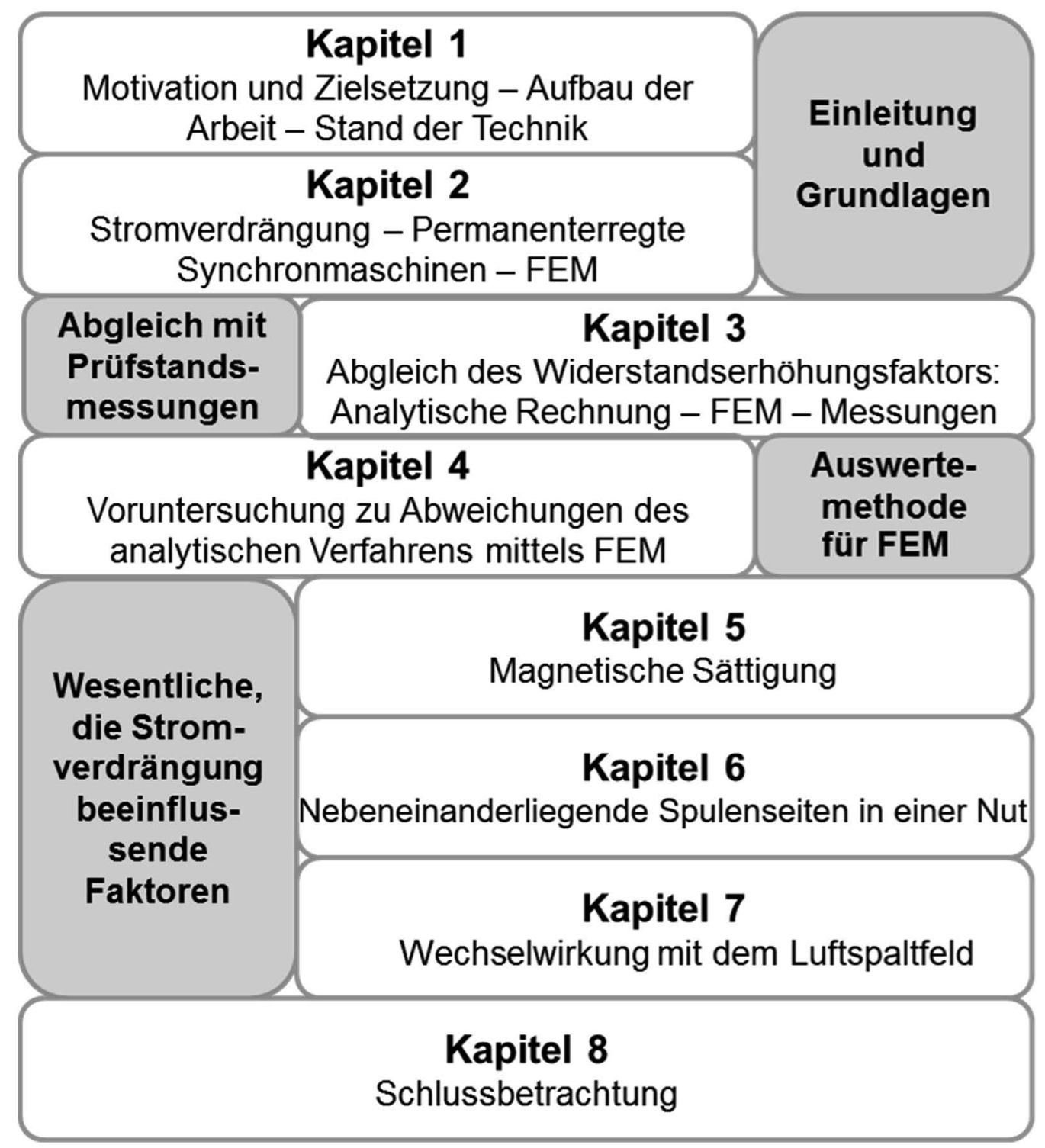

Abbildung 1-1: Aufbau der Arbeit

In Kapitel 2 werden die notwendigen Grundlagen zur Behandlung des Themas vorgestellt. Hierbei wird in kurzer Form in die Themenfelder Stromverdrängung, permanenterregte Synchronmaschinen und Finite-Elemente-Rechnung eingeführt.

Anschließend findet in Kapitel 3 ein Abgleich von verschiedenen Ermittlungswegen zur Bestimmung der Stromverdrängung statt. Dazu wird zunächst die für diesen Zweck verwendete elektrische Maschine vorgestellt, dann wird auf die einzelnen Ermittlungswege eingegangen. Am Ende des Kapitels wird überprüft, ob das Permanentmagnetfeld die Hauptursache für die abweichenden Ergebnisse des analytischen Ermittlungswegs ist. 
In Kapitel 4 wird eine Auswertemethode für Finite-Elemente-Rechnungen entwickelt, um Einflüsse auf die Stromverdrängung prüfen und bewerten zu können. Des Weiteren werden erste Beeinflussungen der Stromverdrängung untersucht.

Kapitel 5 widmet sich der magnetischen Sättigung des Eisenkreises. In einem ersten Schritt wird der Einfluss der magnetischen Sättigung auf das Nutfeld und die Stromverdrängung ermittelt. Im nächsten Schritt wird die magnetische Sättigung der Zähne tiefergehend behandelt. Darüber hinaus wird der Sättigungseffekt an der in Kapitel 3 beschriebenen elektrischen Maschine betrachtet, und in einem letzten Schritt werden die gewonnen Erkenntnisse analytisch formuliert.

Kapitel 6 stellt den Effekt von nebeneinander in der Nut liegenden Spulenseiten auf die Stromverdrängung dar. Dabei wird nach Vorüberlegungen zu den Zusammenhängen eine Untersuchung der Auswirkungen auf das Nutfeld und die Stromverdrängung mittels Finite-Elemente-Berechnungen durchgeführt. Im Anschluss erfolgt eine Betrachtung anhand der elektrischen Maschine aus Kapitel 3 und die Ableitung von analytischen Erkenntnissen.

In Kapitel 7 wird die Wechselwirkung mit dem Luftspaltfeld untersucht. Hierbei wird überprüft, wie sich Feldschwäch- und Konstantflussbereich sowie die Staffelung und die Nutzung des Reluktanzmoments auf die Stromverdrängung auswirken. Die Analysen in diesem Kapitel erfolgen ausschließlich anhand der elektrischen Maschine aus Kapitel 3. Die Zusammenhänge zur Wechselwirkung mit dem Luftspaltfeld werden nicht analytisch formuliert. Stattdessen wird erläutert, wodurch eine solche analytische Darstellung erschwert wird.

Abschließend werden die Ergebnisse der Arbeit in Kapitel 8 zusammengefasst. Zusätzlich werden weiterführende Untersuchungen aufgezeigt, um die Datenbasis der diskutierten analytischen Zusammenhänge zu verbessern und an die gewonnenen Erkenntnisse anzuknüpfen.

\subsection{Stand der Technik}

Der Stand der Technik wird in zwei Teilen aufbereitet. Zunächst wird ein chronologischer Überblick über die Abfolge des Wissenszuwachses zum Thema Stromverdrängung in den Wicklungen elektrischer Maschinen gegeben. Dabei werden wesentliche Schlüsselerkenntnisse berücksichtigt. Im Anschluss wird eine sachbezogene Zusammenfassung der zentralen Erkenntnisse gegeben.

\subsubsection{Chronologische Darstellung}

In diesem Abschnitt werden die relevanten Veröffentlichungen und bisherigen Erkenntnisse zum Thema Stromverdrängung zusammengetragen. Dabei werden die Begriffe Stromverdrängung, Wirbelstrom, Widerstandsverhältnis, Widerstandserhöhungsfaktor und Wechselstromwiderstand verwendet. Eine Differenzierung der Begriffe sowie eine Darstellung der Zusammenhänge folgt in Abschnitt 2.1.5.

Wie bereits in Abschnitt 1.1 kurz erwähnt, reichen die Betrachtungen zur Stromverdrängung in elektrischen Leitern bis Anfang des 20. Jahrhunderts zurück. Erste Ansätze zur Bestimmung des Wechselstromwiderstands, der die Auswirkungen der Stromverdrängung berücksichtigt, stammen aus dem physikalischen Umfeld. Dort gelang es Sommerfeld [55] 1904, die Wechselstromverteilung in einer Spule für alle Frequenzen des Stroms analytisch zu berechnen. 
Ein halbes Jahr später veröffentlichte Field [11] 1905 seine für die Verhältnisse in elektrischen Maschinen wichtige Betrachtung eines Leiters, der an drei Seiten von ferromagnetischem Material umgeben ist - also in einer ferromagnetischen Nut liegt. Field teilt zu Beginn seiner Arbeit die Ursachen für Stromverdrängung in zwei Kategorien auf: Stromverdrängung, die durch das magnetische Feld des betrachteten Leiters oder eines anderen Leiters in der betrachteten Nut bewirkt wird, und Stromverdrängung, die durch äußere magnetische Felder verursacht wird. Im Verlauf seiner Arbeit erwähnt er den in die Nut eindringenden Luftspaltfluss als Ursache zusätzlicher Stromverdrängung. Er nennt aber keine Maßnahmen, um diesen zusätzlichen Verlusten entgegenzuwirken. Field vertritt in seiner Arbeit des Weiteren den Standpunkt, dass die magnetische Sättigung des Eisens keinen signifikanten Einfluss auf die Stromverdrängung haben wird. Dies wird später widerlegt werden. Den Kern seiner Arbeit bildet die analytische Berechnung des Wechselstromwiderstands. Zur Darstellung wählt er bereits das Verhältnis zwischen Wechselstromwiderstand und Gleichstromwidersand - den sogenannten Widerstandserhöhungsfaktor. Seine Berechnung bezieht sich auf einen einzelnen rechteckigen Leiter in einer offenen rechteckigen Nut.

1908 greift Emde [8] das Thema auf und publiziert eine ausführlichere Herleitung der Zusammenhänge. Er verwendet eine etwas andere Schreibweise, die laut Rogowski [51] besser auf die praktische Anwendung zugeschnitten ist. Rogowski fasst in seinem Beitrag die Erkenntnisse Sommerfelds, Fields und Emdes zusammen und zeigt, dass ihre Erkenntnisse sich zum gleichen Formelwerk führen lassen. Darüber hinaus knüpft Rogowski an die Ergebnisse an und betrachtet geschlossene Nuten sowie mehrere Leiter übereinander in der Nut und beschreibt die kritische Kupferhöhe. Die geschlossenen Nuten betrachtet er im Hinblick auf Maschinen mit Zahnköpfen. Er kommt zu dem Ergebnis, dass sich die Betrachtungen für offene Nuten bereits bei kleinen Nutschlitzen auch für Maschinen mit Zahnköpfen anwenden lassen.

Bei der Betrachtung mehrerer Leiter übereinander in der Nut erwähnt Rogowski die Erkenntnis, die bereits in den fieldschen Gleichungen enthalten, aber aufgrund ihrer Auflösung nicht sofort ersichtlich war: Die näher an der Nutöffnung, also weiter oben in der Nut liegenden Leiter sind nicht nur dem eigenen magnetischen Feld ausgesetzt, sondern zusätzlich dem magnetischen Feld der darunter liegenden Leiter. Es lässt sich also beschreiben, dass der von außen angelegte, gleichverteilte Strom in einem weiter oben in der Nut liegenden Leiter von zwei Wirbelströmen überlagert wird: zum einen von einem Wirbelstrom, der durch das magnetische Feld des betrachtenden Leiters selbst verursacht wird, und zum anderen von einem Wirbelstrom, der durch das magnetische Feld der unter dem betrachteten Leiter liegenden Leiter bewirkt wird.

Die letzte Ergänzung Rogowskis in seinem Beitrag beschäftigt sich mit der kritischen Kupferhöhe, die später auch als kritische Leiterhöhe bezeichnet wird beispielsweise bei Müller u.a. [34]. Die kritische Kupferhöhe geht auf das gegensätzliche Verhalten des Gleichstromwiderstands und des Widerstandserhöhungsfaktors zurück. Bei einem Leiter in einer ferromagnetischen Nut nimmt der Gleichstromwiderstand mit größerer Leiterhöhe, also größerem Leiterquerschnitt, ab, während der Widerstandserhöhungsfaktor mit größerer Leiterhöhe zunimmt. Der Wechselstromwiderstand ist das Produkt des Gleichstromwiderstands und des Widerstandserhöhungsfaktors. Rogowski zeigt erstmals auf, das das gegensätzliche 
Verhalten zu einem Minimum des Wechselstromwiderstands bei einer bestimmten Leiterhöhe führt. Diese Höhe hängt von der Frequenz des Stroms und der Anzahl der übereinanderliegenden Leiter $a b$. Wird die kritische Leiterhöhe überschritten, steigen die im Kupfer auftretenden Verluste trotz des höheren Materialeinsatzes an. Aus der kritischen Kupferhöhe leitet Rogowski Erkenntnisse über das Widerstandsverhältnis ab. Er kommt zu dem Ergebnis, dass bei Einhaltung der kritischen Leiterhöhe die Wechselstromverluste einer Spule die Kupferverluste bei homogen verteiltem Strom nie um mehr als 33\% überschreiten. Am Ende seines Beitrags regt Rogowski die Überprüfung der von Sommerfeld, Field und Emde aufgestellten und von ihm ergänzten Ansätze in Versuchen an.

Der Aufforderung Rogowskis folgt Richter [45], indem er 1914 Messungen veröffentlicht, die die Berechnungen grundsätzlich bestätigen. Diese Publikation kommentiert wiederum Rogowski [52]. In seiner Anmerkung betont er, dass es sich bei dem analytischen Ansatz um ein idealisiertes Problem handelt. Die Anwendbarkeit für die Praxis könne nur durch vergleichende Messreihen, wie die von Richter, überprüft werden.

Tiefergehende Betrachtungen zu den Grenzen, ab denen sich die vereinfachende Annahme eines reinen Nutquerfelds nicht mehr ausreichend mit der Wirklichkeit deckt, wurden bisher nicht publiziert.

Von Bedeutung ist auch Rogowskis Kommentar [52] zur kritischen Kupferhöhe. Rogowski sagt, dass eine geringfügige Überschreitung der kritischen Kupferhöhe legitim sein könne. Diese Erkenntnis ergibt sich aus Richters Messungen. Wenn die Dauerleistung der Maschine eine hohe Bedeutung hat und daher das thermische Verhalten mit in den Fokus kommt, wird die Bedeutung der kritischen Kupferhöhe eingeschränkt. Denn dann muss berücksichtigt werden, dass durch die größere Leiterhöhe auch die Leiteroberfläche wächst. Mit zunehmender Leiterfläche verringert sich der thermische Widerstand, da die wärmeabführende Fläche zunimmt. Rogowski vertritt den Standpunkt, dass der geringere thermische Widerstand leicht höhere Kupferverluste überkompensiere und eine geringfügige Überschreitung der kritischen Kupferhöhe damit sinnvoll seien könne.

Richter vertieft im Anschluss an seine erste Veröffentlichung über die Stromverdrängung seine Studien und publiziert von 1915 bis 1917 eine zusammenhängende vierteilige Reihe von Aufsätzen zu dem Thema. In dieser geht er ausführlich auf Möglichkeiten zur Unterdrückung der Stromverdrängung ein und betrachtet neben den schon bekannten Rechteckleitern weitere Leitergeometrien. Die Betrachtungen basieren auf der idealisierenden Annahme eines reinen Nutquerfelds. Richter erwähnt in seiner Arbeit kurz, dass darüber hinaus auch das in die Nuten eindringende Hauptfeld zusätzliche Stromverdrängung verursachen könne und dass breitere Leiter dafür anfälliger seien. Dies betreffe aber in der Regel nur die oberste Leiterschicht in der Nut, und bei Zahnköpfen seien die Auswirkungen vernachlässigbar. An diesen Aspekt wird die vorliegende Arbeit im weiteren Verlauf anknüpfen.

Im ersten Teil seiner Reihe [47] betrachtet Richter den Einfluss der Leitfähigkeit auf den Stromverdrängungseffekt. Dabei untersucht er die Beeinflussung durch das Material des Leiters und dessen Temperatur. Er kommt beispielsweise zu dem Ergebnis, dass sich bei Verwendung von Aluminiumleitern trotz des schlechteren Leitwerts die resultierenden Verluste in den oberen Leiterlagen einer Wicklung gegenüber einer vergleichbaren Kupferwicklung reduzieren ließen. Die Ursache liegt in der geringeren 
Ausbildung der Stromverdrängung und dem damit sinkenden Widerstandserhöhungsfaktor.

Der zweite Teil [46] der Richter-Serie widmet sich der Betrachtung unterschiedlicher Leitergeometrien und der Anordnung der Leiter für eine optimale Wärmeabfuhr. Neben dem bekannten rechteckigen Querschnitt berechnet Richter in dieser Arbeit die Stromverdrängung in Leitern mit quadratischem und kreisförmigem Querschnitt. Er zeigt auf, dass das Temperaturverhalten von Aluminiumspulen mit kreisförmigem oder quadratischem Querschnitt näher an das von Kupferspulen herankommt, als dies bei rechteckigem Querschnitt der Fall ist. Außerdem behandelt er eine Leiteranordnung für eine optimale Wärmeabfuhr. Dafür wird die Nut im oberen mittleren Bereich nicht mit der Wicklung gefüllt. Stattdessen wird dort ein Füllstück eingesetzt, um das die Wicklung herumgewickelt wird. Dadurch wird eine große Oberfläche für die Wärmeabfuhr erreicht.

Richters dritter Beitrag [48] zur Stromverdrängung befasst sich mit unterteilten Leitern und insbesondere deren Verschränkung zur Unterdrückung der durch das Nutquerfeld hervorgerufenen Stromverdrängung. Die verschränkten Ankerstäbe werden später auch als Kunststäbe oder, nach inrem Erfinder, als Roebelstäbe bezeichnet. Richter zeigt, dass sich Stromverdrängung durch eine geeignete Verschränkung der Leiter sehr wirksam verringern lässt. Die Maßnahmen hält er insbesondere im Zusammenhang mit großen Maschinen für sinnvoll. In der Veröffentlichung unterscheidet er auch erstmals Stromwärme ersten Grades und zweiten Grades. Dabei meint Stromwärme ersten Grades Wirbelströme, die sich über mehrere Leiter erstrecken, während Stromwärme zweiten Grades Wirbelströme beschreibt, die sich innerhalb eines Leiters schließen. Die Unterscheidung ist auch heute noch mit den Begriffen Stromverdrängung erster und zweiter Ordnung gebräuchlich [34].

Im vierten und letzten Teil seiner Serie [49] behandelt Richter magnetische Hilfskreise zur Unterdrückung von zusätzlichen Verlusten durch Wirbelströme. Dabei stellt er drei Arten von magnetischen Hilfskreisen vor: U-förmige Eisensegmente, die auf den Wickelkopf aufgeschoben werden, Blechstreifen, die zwischen den Leiterlagen in die Nut gelegt werden, und Drosselspulen, die in den wicklungsbildenden Leiter integriert werden. Durch diese Hilfskreise ist ebenfalls eine Verringerung des Wechselstromwiderstands möglich. Die Reduktion des Drehmoments durch diese Maßnahmen wird nicht thematisiert. Beispielsweise verursachen die in die Nut eingelegten Blechstreifen je nach Dicke einen magnetischen Kurzschluss der Zähne.

Als Gesamtfazit seiner vierteiligen Betrachtung formuliert Richter, dass die Stromverdrängung durch geeignete Verschränkung der Leiter innerhalb der Nut und durch passende magnetische Hilfskreise vollkommen unterdrückt werden könne. Für eine mit den genannten Maßnahmen sinnvoll entworfene Wicklung gebe es keine kritische Leiterhöhe mehr.

1920 greift Gilman [15] die fieldsche Einteilung der magnetischen Felder, die Stromverdrängung verursachen, wieder auf. So lasse sich kategorisieren, dass Stromverdrängung zum einen durch das Nutquerfeld und zum anderen durch das in die Nuten eindringende Hauptfeld verursacht werde. Gilman zeigt zwar keinen analytischen Ansatz zur Berechnung der durch das Hauptfeld hervorgerufenen Stromverdrängung auf, er gibt aber Hinweise, wie sich Stromverdrängung durch das Hauptfeld vermeiden lasse. Dies sei mit Hilfe von tief in den Nuten sitzenden Spulen und insbesondere mit Zahnköpfen im Statorblechschnitt möglich. Des Weiteren er- 
wähnt er die magnetische Flussdichte in den Zähnen. Diese solle gering gehalten werden, um ein Eindringen des Hauptfelds in die Nuten zu vermeiden.

1922 zeigt Emde [9] noch einmal die Herleitung der beiden zur Bestimmung des Widerstandserhöhungsfaktors notwendigen Hilfsfunktionen $\varphi$ und $\psi$ auf. Dabei legt er das Hauptaugenmerk darauf, dies in möglichst knapper Form zu erreichen.

1933 erweitert Laible [29] den Wissenstand um die analytische Berechnung der Stromverdrängung in trapezförmigen und dreieckigen Leitern, die in trapezförmige Nuten eingebettet sind. Er zeigt, dass Leiter mit zunehmender Dreiecksform der Nut stärker von Stromverdrängung betroffen sind als Leiter gleicher Höhe in rechteckigen Nuten. Er stellt den Verlauf des Widerstandserhöhungsfaktors in einem Diagramm für verschiedene Geometrien gegenüber.

Dwight [7] veröffentlicht 1945 Untersuchungen zur Stromverdrängung. Dabei betrachtet er den Widerstandserhöhungsfaktor über die Frequenz für unterschiedliche Geometrien und Verhältnisse. Er geht auch auf die bereits bei Richter behandelte Verschränkung ein sowie auf unterschiedliche Leiteranordnungen, wie beispielsweise unterschiedliche Stränge in einer Nut. Des Weiteren befasst er sich auch mit der Abführung der im Leiter entstehenden Wärme.

1963 entwickelt Oberretl [35] einen alternativen Weg zur Bestimmung der Stromverdrängung. Er schlägt die Berechnung mit Hilfe von Gittermodellen vor. Dabei handelt es sich um Schaltungen aus Kondensatoren und Widerständen, die passend zum jeweiligen Problem ausgewählt und geschaltet werden sollen. Anhand von Messungen an diesem Versuchsaufbau soll auf die realen elektromagnetischen Verhältnisse zurückgeschlossen werden. Der Ansatz bleibt exotisch, da sich bald durch die voranschreitende Computertechnik neue Möglichkeiten zur Bestimmung der Stromverdrängung mittels numerischer Methoden ergeben.

Ein weiterer Sonderfall der Stromverdrängung wird 1966 von Reichert [43] betrachtet. Er berechnet den Wechselstromwiderstand für Hohlleiter, wie sie beispielsweise als wassergekühlte Leiter in Turbogeneratoren zum Einsatz kommen.

Richter [50] fasst in seinem umfassenden Werk über elektrische Maschinen seine Erkenntnisse zur Stromverdrängung zusammen. Er gibt analytische Ansätze für verschiedene einfache Modelle an, beispielsweise für das in die Nuten eindringende Luftspaltfeld.

Im Jahr 1969 publiziert Oberretl zwei weitere Arbeiten mit Bezug zur Stromverdrängung. Die erste Arbeit tangiert das Thema nur. Es handelt sich um eine Anleitung mit 13 Regeln, wie sich zusätzliche Verluste in Induktionsmaschinen reduzieren lassen [36]. Regel 7 betrifft dabei auch die Leitergeometrie im Stator: Der Abstand des obersten Leiters zum Luftspalt soll seiner Ansicht nach ein Drittel der Nutbreite betragen. Für den Rotor empfiehlt er sogar ein Verhältnis von 1 zu 1. Hiervon weicht er beim Stator mit Rücksicht auf den Kupferfüllfaktor ab. Die Abstandsregel ist nach Oberretls Ansicht nur bei offenen Nuten mit einem Rechteckleiter pro Ebene notwendig. Bei zwei nebeneinanderliegenden Leitern pro Ebene oder einem Stator mit Zahnköpfen sei kein Abstand notwendig. In seiner zweiten Veröffentlichung aus diesem Jahr stellt er eine Anpassung seines Gittermodells vor [37]. Mit Hilfe von ausgewählten Silizium-Zener-Dioden soll das Verhalten von sättigendem Eisen nachgebildet und in der Auswertung berücksichtigt werden. In einem Diagramm stellt er den Anstieg des Widerstandserhöhungsfaktors über die magnetische Flussdichte im Luftspalt dar. Er bezeichnet den starken Anstieg ab einer Flussdichte 
von 1,9 T als bemerkenswert. Zu möglichen Ursachen dieses Anstiegs äußert sich Oberretl hier noch nicht.

Eine Schaltung zur erregungsunabhängigen Betrachtung von Vorgängen im Leiter liefert 1973 Mocanu [31]. Mit Hilfe seiner Kettenleiter-Ersatzschaltung können Übergangszustände in der elektrischen Maschine betrachtet werden.

Stoll [56] liefert 1974 eine Übersicht analytischer Ansätze zur Stromverdrängungsbestimmung für unterschiedliche ein- und zweidimensionale Grundprobleme. Er betont, dass mathematische Modelle schnell zu kompliziert werden, um sie analytisch zu handhaben. In diesen Fällen werde eine numerische Berechnung notwendig, die jedoch die zugrundeliegenden Zusammenhänge verschweige

Im gleichen Jahr beschäftigt sich Schunk [54] mit der grundsätzlichen Erscheinung der Stromverdrängung. In einem kleinen Kapitel behandelt er Wechselstrommaschinen. Darin leitet er in übersichtlicher Form die ein- und zweidimensionale Stromverdrängung sowie die dafür notwendigen Funktionen $\varphi(\beta)$ und $\psi(\beta)$ her.

Eine erste Veröffentlichung über Finite-Elemente-Berechnungen zur Bestimmung der Stromverdrängung stammt von Chari u. a. [5] aus dem Jahr 1977. Darin wird die analytische Berechnung der Stromverdrängung mit einer zweidimensionalen FiniteElemente-Rechnung abgeglichen. Es werden vergleichbare Ergebnisse erzielt. Bei flachen Leitern ergeben sich jedoch zunehmende Abweichungen. Die Annahme eines reinen Nutquerfelds wird in der Arbeit nicht als mögliche Ursache dieser Abweichungen betrachtet.

Oberretl [38] ergänzt den Wissensstand 1978 durch eine Gleichung. Diese dient der Bestimmung der zusätzlichen Stromverdrängungsverluste durch das in die Nuten eindringende Hauptfeld. Für die Lösung nutzt er das von ihm entwickelte Gitteranalogie-Verfahren [35] [37]. Die Gleichung liefert die zusätzlichen Verluste für die gesamte Nut und nicht für die einzelnen Leiterlagen. Aufgrund der vorherrschenden Verhältnisse könne man die Verluste algebraisch zu den Verlusten durch das Nutquerfeld hinzuaddieren. Oberretl berücksichtigt bei seinem Ansatz, dass die Eisensättigung in den Zahnkanten nicht vernachlässigt werden kann. Zum Ende seines Beitrags widmet er einen kurzen Abschnitt der magnetischen Sättigung der Zähne. Er erwähnt darin, dass durch eine hohe mittlere Zahninduktion ein Nutlängsfeld in der Nut entsteht. Dieses ruft ebenfalls Wirbelströme hervor, die ab einer Zahninduktion von 1,8 T von Bedeutung seien. Ein Einfluss der Leiterbreite auf die Anfälligkeit des Leiters für Längsfelder wird nicht erwähnt.

Einen weiteren Ansatz zur Berechnung der zusätzlichen Verluste durch das in die Nuten eindringende Hauptfeld liefert 1988 Dabrowski u. a. [6]. Seine Formel basiert auf Erkenntnissen aus Finite-Elemente-Berechnungen. Die aufgestellte Gleichung soll im Vergleich zu anderen Gleichungen akkurate Ergebnisse liefern, wobei explizit Oberretls Arbeit [38] aus dem Jahre 1978 genannt wird.

2005 beschäftigt sich Mosebach [32] mit der Auswirkung von Zahnspulen auf die Stromverdrängung. Es handelt sich um eine den ursprünglichen Ansatz von Sommerfeld, Field und Emde ergänzende Betrachtung. Dabei wird der Fall betrachtet, dass zwei Spulenseiten nebeneinander in einer Nut liegen. Die Bestimmung des Einflusses erfolgt in weiten Teilen analytisch. Lediglich die Integration der Verluste erfolgt auf numerischem Weg. Das Resultat ist ein Vorfaktor für den Widerstandserhöhungsfaktor. Dabei zeigt sich, dass eine Abhängigkeit dieses Faktors von der Phasendifferenz der Ströme in den beiden Spulenseiten besteht. Mit zunehmender Phasendifferenz der Ströme in den benachbarten Spulenseiten reduziert sich der Faktor. Die Höhe der 
Abnahme ist wiederum vom ursprünglichen Widerstandserhöhungsfaktor abhängig. Es wird ersichtlich, dass die Stromverdrängung durch das Nutquerfeld mit zunehmender Phasendifferenz reduziert wird. Dazu passende Ergebnisse finden sich auf Basis von Finite-Elemente-Rechnungen auch bei Gröninger u. a. [16]. Auf Unterschiede in den Ergebnissen wird im weiteren Verlauf der vorliegenden Arbeit eingegangen.

Eine ausführliche Betrachtung der räumlichen Diskretisierung als Berechnungsweg der Stromverdrängung findet sich 2009 bei Köhring [27]. Kern seiner Arbeit ist die Betrachtung der Stromverdrängung in manuell geträufelt ausgeführten Drehstromankerwicklungen. Dabei werden die Auswirkungen unterschiedlicher Leiterverteilungen in der Nut untersucht. In einem Kapitel werden auch die Folgen eines gesättigten Eisenkreises auf die nutquerfeldbedingte Stromverdrängung behandelt. Köhring berücksichtigt diese analytisch mit Hilfe eines veränderlichen Permeabilitätswerts des Eisens. Der Widerstandserhöhungsfaktor nimmt bei seiner Finite-Elemente-Rechnung mit zunehmender Eisensättigung ab, was im Widerspruch zu Oberretls Beobachtungen von 1978 steht. Dieser Aspekt wird in der vorliegenden Arbeit aufgegriffen.

2013 befasst sich Endert u. a. [10] mit Stromverdrängung im Zusammenhang mit Spulen mit besonders hohem Kupferfüllfaktor. Dabei werden unterschiedliche Geometrien und Leiteranordnungen mittels Finite-Elemente-Rechnungen untersucht. Ein interessanter Aspekt ist die Betrachtung von zwei Spulenseiten in einer Nut, wobei die Anordnung als Zahnspulen, also mit in der Nut nebeneinanderliegenden Spulenseiten, höhere zusätzliche Verluste zur Folge hat als die Anordnung der beiden Spulenseiten übereinander in der Nut. Diese Erkenntnis widerspricht scheinbar den Ergebnissen Mosebachs [32] und Gröningers u.a. [16], worauf im Verlauf der vorliegenden Arbeit noch eingegangen wird.

Gröninger u.a. [16] beschäftigt sich 2014 ebenfalls mit Stromverdrängung im Zusammenhang mit Spulen von großem Leiterquerschnitt und hohem Kupferfüllfaktor. Die beschriebenen gegossenen Spulen zeichnen sich durch flache Leiter aus. Dies beugt der Stromverdrängung durch das Nutquerfeld vor. Der mittels Finite-ElementeRechnungen bestimmte Widerstandserhöhungsfaktor liegt jedoch signifikant oberhalb des analytisch berechneten. Hier wird seitens Gröningers eine mögliche Beeinflussung durch rotierende Permanentmagnete, magnetische Sättigung des Statoreisens und zwei Spulenseiten in einer Nut vermutet. Der Einfluss der Phasenverschiebung auf die Stromverdrängung wird in zu Mosebach [32] vergleichbarer Form dargestellt, wobei die Ergebnisse bei Gröninger aus Finite-Elemente-Rechnungen stammen. In weiten Teilen deckt sich deren Aussage mit Mosebach: Die Stromverdrängung nimmt mit zunehmender Phasenverschiebung ab. Lediglich bei dem betrachteten flachen, breiten Leiter nimmt die Stromverdrängung mit zunehmender Phasenverschiebung zu. Die Ursache dieser Abweichung wird im späteren Verlauf der hier vorliegenden Arbeit noch behandelt. Des Weiteren wird von Gröninger dargestellt, dass die Verluste der linken und der rechten Spulenhälfte in der Nut in Abhängigkeit vom Betriebspunkt unterschiedlich hoch sind. Darüber hinaus wird die Abhängigkeit der Stromverdrängung von der Temperatur des Leiters dargestellt, wie sie auch bei Richter [47] behandelt wird. Als Maßnahmen zur Reduktion der Stromverdrängung werden das Vergraben der Magnete im Rotor sowie die Verwendung von Zahnköpfen im Statorblechschnitt vorgeschlagen. Beides beugt der Stromverdrängung vor, die durch das Eindringen des Hauptfelds in die Nut bewirkt wird. 


\subsubsection{Sachbezogene Darstellung}

Die Essenz aus den bisherigen Veröffentlichungen kann folgendermaßen zusammengefasst werden:

Mit dem von Sommerfeld [55], Field [11] und Emde [8] entwickelten Formelwerk zur Berechnung des Widerstandserhöhungsfaktors gibt es eine seit über einhundert Jahren bewährte Grundlage zur Bestimmung der Stromverdrängung. Diese beruht auf der Annahme eines reinen Nutquerfelds und weiteren Randbedingungen wie unendlich tiefen Nuten. Die ursprüngliche Betrachtung eines rechteckigen Leiters in einer offenen, rechteckigen Nut wurde um weitere analytische Berechnungen ergänzt. So wurden runde [46] und quadratische [46] Leiterquerschnitte betrachtet, sowie Hohlleiter [43], Trapeznuten [29] und geschlossene Nuten [51] und des Weiteren zwei nebeneinander in einer Nut liegende Spulenseiten [32] und Ansätze zur Berücksichtigung der Eisensättigung [6] [27]. Neben diesen analytischen Betrachtungen, die stets von einem reinen Querfeld in der Nut ausgehen, gab es von Anfang an die Ansicht, dass es weitere Ursachen für Stromverdrängung gibt.

Immer wieder taucht in der Literatur das als Längsfeldkomponente in die Nuten eindringende Hauptfeld auf [6] [11] [15] [36] [38] [46] [50], das zusätzliche Stromverdrängungsverluste verursache. Davon seien aber nur die oberen Leiterlagen in der Nut betroffen [15] [36] [38] [46]. Es wird auch erwähnt, dass breite Leiter anfälliger für das eindringende Hauptfeld seien [38] [46]. Zur Vermeidung zusätzlicher Verluste durch das Längsfeld finden sich häufig Handlungsempfehlungen. Eine Möglichkeit, den Folgen durch die Längsfeldkomponente vorzubeugen, sei ausreichend Abstand der Spulen zur Nutöffnung [15] [36] [38] [50]. Des Weiteren sei eine wirksame Unterdrückung der zusätzlichen Verluste auch mit Hilfe von Zahnköpfen im Statorblechschnitt möglich. Diese sollen das Hauptfeld am Eindringen in die Nut hindern. Zu diesem Ansatz besteht ein breiter Konsens [15] [16] [36] [46] [50]. Vereinzelt wird auch die magnetische Sättigung des Stators als Einfluss ausgemacht, die es zu vermeiden gilt [15] [38].

Es finden sich einige wenige analytische Ansätze zur Bestimmung der zusätzlich entstehenden Verluste durch das in die Nuten eindringende Hauptfeld [6] [38] [50]. Diese Ansätze sind jedoch teilweise widersprüchlich und konnten sich in der Praxis nicht durchsetzen. In der Regel wird daher auf die im Abschnitt zuvor genannten Handlungsempfehlungen verwiesen.

Der Stand der Literatur liest sich, als ob sich die Auswirkungen der Stromverdrängung durch flache Leiter und Zahnköpfe im Statorblechschnitt wirksam unterdrücken ließen. Jedoch gibt es Fälle, die von dieser Regel abzuweichen scheinen [10] [16] [37]. Diesen soll im Rahmen der vorliegenden Arbeit auf den Grund gegangen werden.

Neben den genannten Maßnahmen erforscht insbesondere Richter noch eine Vielzahl an Möglichkeiten zur Verringerung und Vermeidung von Stromverdrängung. Hierbei seien insbesondere die Verschränkung und die magnetischen Hilfskreise genannt. Die Anwendbarkeit dieser Lösungen sieht er jedoch eher bei großen Maschinen als gegeben an [48] [49]. 
1 Einleitung 


\section{Grundlagen}

In den Grundlagen wird zunächst das notwendige Fundament zur Behandlung der Stromverdrängung dargestellt (Abschnitt 2.1). Des Weiteren gibt der Abschnitt „Permanenterregte Synchronmaschinen" das grundlegende Wissen zu elektrischen Maschinen wieder, welches zum Verständnis der weiteren Ausführungen notwendig ist (Abschnitt 2.2). Abschließend werden die Grundlagen der Finite-ElementeBerechnung kurz vorgestellt (Abschnitt 2.3).

\subsection{Stromverdrängung}

In diesem Abschnitt wird, ausgehend von den Verhältnissen ohne Stromverdrängung (Abschnitt 2.1.1), die Ursache der Stromverdrängung erläutert. Diese ergibt sich aus den Maxwell-Gleichungen (Abschnitt 2.1.2). In Abschnitt 2.1.3 wird dargestellt, wie Materialien, insbesondere ferromagnetische Materialien, die Stromverdrängung beeinflussen. Der Abschnitt 2.1.4 widmet sich der analytischen Berechnung der Stromverdrängung in elektrischen Maschinen. Abschließend werden Begriffe zur Stromverdrängung erläutert und voneinander abgegrenzt (Abschnitt 2.1.5).

\subsubsection{Verhältnisse ohne Stromverdrängung}

Als Ausgangspunkt werden die Verlustverhältnisse in elektrischen Spulen für den Fall betrachtet, dass keine Stromverdrängung vorliegt. Dies gilt bei der Verwendung von Gleichstrom. In diesem Fall lassen sich die Verluste in der Spule mit dem ohmschen Gesetz und der elektrischen Leistung nach

$$
P_{v}=I_{g}^{2} R
$$

bestimmen. Die Gleichstromverluste $P_{v}$ sind proportional zum elektrischen Widerstand $R$ und zum Quadrat des elektrischen Gleichstroms $I_{g}$. Der elektrische Widerstand hängt wiederum von Eigenschaften des für die Spule verwendeten elektrischen Leiters ab gemäß

$$
R=\rho \frac{l_{l}}{A_{l}} .
$$

Die widerstandsbestimmenden Eigenschaften des Leiters sind der spezifische elektrische Widerstand $\rho$ des verwendeten Materials, die Länge des Leiters // und die Querschnittsfläche des Leiters $A$.

Unter Verwendung der Stromdichte Jlässt sich der Strom ausdrücken durch

$$
I=A_{l} J
$$


Gleichung (2-3) gilt, sofern die Querschnittsfläche senkrecht vom Strom durchflossen wird und die Stromdichte im gesamten Querschnitt konstant ist. Mit ihr lässt sich aus den Gleichungen (2-1) und (2-2) der Zusammenhang

$$
P_{v}=\rho A_{l} J^{2} l_{l}
$$

darstellen. In Gleichung (2-4) ist die Stromdichte $J$ die einzige Größe, die quadratisch in die elektrische Verlustleistung eingeht. Verteilt sich der Strom aus irgendeinem Grund ungleichmäßig über die Querschnittsfläche, ist die Berechnung der Verlustleistung nach Gleichung (2-1) nicht zulässig. Dann müsste die elektrische Verlustleistung für jeden Teilbereich mit homogener Stromdichte nach Gleichung (2-4) separat berechnet werden. Die resultierende Verlustleistung ergibt sich dann aus der Summe der Teilverlustleistungen. Bei gegebenem Leiter und Strom nimmt die resultierende Verlustleistung mit zunehmender Inhomogenität der Stromdichte zu. Stromverdrängung verursacht eine solche inhomogene Stromdichteverteilung. Die Zusammenhänge werden in Abschnitt 2.1.2 erläutert.

\subsubsection{Maxwell-Gleichungen}

Der gängige Erklärungsansatz für das Phänomen der Stromverdrängung, wie er sich beispielsweise bei Müller u.a. [34] und Küpfmüller [28] findet, lautet wie folgt: Wenn sich ein elektrisch leitender Stoff in einem magnetischen Wechselfeld befindet, entstehen nach dem Induktionsgesetz

$$
\operatorname{rot} \vec{E}=-\frac{\partial}{\partial t} \vec{B}
$$

elektrische Feldstärkewirbel. Nach dem ohmschen Gesetz sind die elektrische Feldstärke $E$ und die elektrische Stromdichte $J$ gemäß

$$
J=\kappa E
$$

verknüpft. Dadurch führt der elektrische Feldstärkewirbel zu einem Wirbelstrom im elektrisch leitenden Stoff. In elektrischen Maschinen wird das auf die Leiter einwirkende magnetische Feld nach dem Durchflutungsgesetz unter anderem durch den im Leiter fließenden Strom gemäß

$$
\operatorname{rot} \vec{H}=\vec{J}
$$

selbst aufgebaut. In Gleichung (2-7) ist der Verschiebungsstrom aufgrund der relativ langsamen Vorgänge bereits vernachlässigt worden.

Die durch das magnetische Wechselfeld bewirkten Wirbelströme überlagern sich dem Leiterstrom. Die Überlagerung führt zu einer ungleichmäßig über den Leiterquerschnitt verteilten Stromdichte, die Stromverdrängung genannt wird. Wie bereits in Abschnitt 2.1.1 angedeutet wurde, führt dies zu höheren Verlusten. Diese zusätzli- 


\subsection{Stromverdrängung}

chen Verluste werden Wirbelstromverluste genannt. Die Erhöhung der Wicklungsverluste kann auch als eine scheinbare Vergrößerung des Wicklungswiderstands aufgefasst werden. Stromverdrängung ist in der Regel unerwünscht. Teilweise wird sie in der Auslegung aber auch gezielt genutzt - beispielsweise zur Vergrößerung des Anzugsmoments bei Induktionsmaschinen mit Käfigläufer.

Der eben beschriebene Erklärungsansatz mit Hilfe von Wirbelströmen hat nach Föppl [13] den Charme, dass für das Verständnis keine gekoppelte Vorstellung von Magnetismus und Elektrizität erforderlich ist. Das magnetische Feld kann als Einfluss außerhalb des Systems verstanden werden, der die Stromverteilung im Leiter beeinflusst. Wie bereits Rogowski [51] anmerkt, liegt die Schwäche dieser Modellvorstellung in der fortgesetzten Korrektur. Als Ausgangspunkt wird der Gleichstromfall betrachtet, der für die vorliegenden Verhältnisse nur näherungsweise zutrifft. Zur Korrektur ist die Berücksichtigung der Wirbelströme notwendig. Jedoch hat der Wirbelstrom wiederum eine Rückwirkung auf das magnetische Feld, wodurch sich ein weiterer Wirbelstrom 2. Ordnung der Stromverteilung überlagert. Dieser Vorgang setzt sich endlos fort. In dieser endlosen Überlagerung liegt die Schwäche der künstlichen Zerlegung des Wechselstroms in homogen verteilten Strom und Wirbelstrom. So hält es auch Sommerfeld [55], der sich auf die maxwellsche Sichtweise beruft, nach der es ein einheitliches Stromfeld und ein durch Beziehungen mit ihm verknüpftes einheitliches Magnetfeld gibt. Eine ungleichmäßige Stromdichteverteilung ergibt sich daraus von selbst durch gewisse Randbedingungen.

Für die maxwellsche Sichtweise ist aber eine andere Modellvorstellung erforderlich, wie sie beispielsweise bei Föppl [13] und Lehner [30] beschrieben wird. Diese Modellvorstellung bezieht das magnetische Feld in das betrachtete System mit ein und hat als Grundlage den Poynting-Vektor

$$
\vec{S}=\vec{E} \times \vec{H}
$$

Der Poynting-Vektor ist das Kreuzprodukt von elektrischer und magnetischer Feldstärke. Er gibt die Dichte und die Richtung der durch das elektromagnetische Feld transportierten Energie an. Bezogen auf einen elektrischen Leiter geschieht dabei der Energietransport nicht im Leiter, sondern im den Leiter umgebenden Dielektrikum. Im Leiter entsteht joulsche Wärme. Die dafür erforderliche Energie wird dem elektromagnetischen Feld entzogen. Dies macht sich als Dämpfung und geringfügige Verzögerung des elektromagnetischen Felds bemerkbar. Wie tief der Leistungsdichtefluss in den Leiter eindringen kann, ist dabei von der Frequenz der Vorgänge, der Beschaffenheit des elektrischen Leiters und den magnetischen Eigenschaften des ihn umgebenden Materials abhängig, wie Lehner [30] auf den Seiten 384ff darstellt. Man spricht in diesem Zusammenhang von Eindringtiefe und Felddiffusion. Je geringer die Eindringtiefe wird oder je weniger tief das Feld in den Leiter hinein diffundieren kann, desto ungleichmäßiger wird die Stromverteilung im Leiter. Die Eindringtiefe bestimmt sich gemäß Binder [3] nach

$$
d_{E}=\sqrt{\frac{b_{n}}{\pi f \mu_{0} \kappa b_{l}}} .
$$


Auch die auf Basis des Poynting-Vektors beschriebenen Zusammenhänge sind letztlich nur eine Modellvorstellung, wie Backhaus [1] verdeutlicht. Aus dieser Modellvorstellung wird jedoch ersichtlich, dass auch die Beschaffenheit des magnetischen Kreises die Stromverdrängung wesentlich beeinflusst. Im folgenden Abschnitt werden die sich daraus ergebenen, für diese Arbeit relevanten Grundlagen dargestellt.

\subsubsection{Eigenschaften des magnetischen Kreises}

Wie im vorhergehenden Abschnitt angedeutet wird, haben die magnetischen Eigenschaften der den elektrischen Leiter umgebenden Materialien Einfluss auf die Stromverdrängung im Leiter.

Der Zusammenhang von magnetischer Feldstärke und magnetischer Flussdichte im Vakuum ist

$$
\vec{B}=\mu_{0} \vec{H}
$$

Liegt kein Vakuum vor, muss der Einfluss der Materialien auf das Verhältnis berücksichtigt werden. Hier weisen ferromagnetische Materialien eine Besonderheit auf: Sie beeinflussen das Verhältnis zwischen magnetischem Feld und magnetischer Flussdichte besonders stark, während beispielsweise paramagnetische Stoffe wie Luft kaum einen Einfluss darauf haben

Der Materialeinfluss auf die magnetische Flussdichte wird in physikalischen Zusammenhängen durch die Ergänzung der Magnetisierung $M$ berücksichtigt gemäß

$$
\vec{B}=\mu_{0}(\vec{H}+\vec{M})=\mu \vec{H}
$$

Die magnetische Polarisation $J_{\text {mag }}=\mu_{0} M$ beschreibt die inneren Magneteigenschaften eines Werkstoffs ohne den magnetischen Flussanteil der Luft. In technischen Anwendungen ist die Verwendung der relativen Permeabilität $\mu_{r}$ üblich, mit der sich formulieren lässt

$$
\mu=\mu_{r} \mu_{0} .
$$

Ferromagnetische Stoffe weisen einen geringen magnetischen Widerstand auf. Eben diese Eigenschaft macht sie interessant für elektrotechnische Anwendungen, beispielsweise in elektrischen Maschinen. Hier liegt aber auch der Einfluss auf die Stromverdrängung. Denn wie aus Gleichung (2-7) ersichtlich ist, geht der elektrische Strom mit einem magnetischen Wirbelfeld einher.

Durch ferromagnetisches Material in der Umgebung eines stromführenden Leiters wird eine höhere magnetische Flussdichte erreicht. Aus Gleichung (2-5) geht hervor, dass die Ursache von Stromverdrängung die Änderung der magnetischen Flussdichte ist. Eine höhere Amplitude der magnetischen Flussdichte führt bei konstantem Stromfluss tendenziell zu höherer Stromverdrängung. Dadurch sind Leiter in elektrischen Maschinen tendenziell stärker von Stromverdrängung betroffen als Leiter, die nur von Luft umgeben sind. 


\subsection{Stromverdrängung}

Die Wirkung ferromagnetischer Materialien auf die magnetische Flussdichte ist begrenzt. Ab einer bestimmten Feldstärke sind alle Elementarmagnete in einem Material ausgerichtet, und die Magnetisierung liefert keinen zusätzlichen Beitrag mehr zur magnetischen Flussdichte. Ab diesem Punkt entsprechen die Verhältnisse wieder der Gleichung (2-10). Dieser Zustand wird magnetische Sättigung genannt. Auch vor Eintritt der Sättigung ist der Zusammenhang zwischen Magnetisierung und magnetischer Flussdichte nur näherungsweise linear. Daher ist auch $\mu_{r}$ keine konstante Größe. Weiterführende Informationen dazu finden sich bei Küpfmüller [28] und Paul [40].

Über eine Analogie zum elektrischen Stromkreis besteht eine weitere Möglichkeit, den Einfluss ferromagnetischer Werkstoffe auf die Stromverdrängung zu verstehen. Die magnetische Umlaufspannung $V_{o}$ entspricht der Durchflutung $\Theta$ und damit der nach Rechtsschraubenzuordnung vorzeichenbehafteten Summe der vom Integrationsweg des Umlaufintegrals eingeschlossenen Ströme (vergleiche Müller u.a. [27]) entsprechend

$$
\Theta=V_{0}=\oint \vec{H} \cdot d \vec{s}=\int \vec{J} \cdot d \vec{A}=\sum_{v z b} i
$$

Der magnetische Widerstand bestimmt sich zu

$$
R_{m}=\frac{l}{\mu_{0} \mu_{r} A}
$$

Ferromagnetische Materialien weisen aufgrund der hohen relativen Permeabilität $\mu_{r}$ einen tendenziell geringen magnetischen Widerstand auf. Im Verhältnis zu vergleichbaren Luftstrecken ist der magnetische Widerstand ferromagnetischer Materialien im Normalfall vernachlässigbar.

In Analogie zum elektrischen Stromkreis stellt der magnetische Fluss

$$
\phi=\frac{\Theta}{R_{m}}
$$

den magnetischen Strom dar. In einem magnetischen Kreis mit ferromagnetischen Abschnitten kommt es zu relativ hohen magnetischen Flüssen. Die Ursache ist der geringe magnetische Widerstand der ferromagnetischen Abschnitte. Ein hoher magnetischer Fluss kann wiederum stärkere Wirbelströme verursachen.

Der Zusammenhang zwischen magnetischer Flussdichte und magnetischem Fluss ist

$$
\phi=\int_{A} \vec{B} d \vec{A}
$$




\subsubsection{Analytische Berechnung}

Wie in Abschnitt 2.1.1 dargestellt, ist Gleichung (2-1) bei Verwendung von Wechselstrom nicht ohne Weiteres zulässig zur Bestimmung der Verlustleistung. Will man die Beziehung weiterhin verwenden, muss der Widerstand $R$ so definiert werden, dass die Verlustvergrößerung enthalten ist. Dies geschieht mit Hilfe des sogenannten Widerstandsverhältnisses

$$
k_{r}=\frac{P_{w}}{P_{g}}=\frac{R_{w}}{R_{g}},
$$

das auch als Widerstandserhöhungsfaktor bezeichnet wird. Aus dem Widerstandsverhältnis ist ersichtlich, dass die Verlustleistung bei Wechselstrom $P_{w}$ der Verlustleistung bei Gleichstrom $P_{g}$ multipliziert mit dem Widerstandserhöhungsfaktor $k_{r}$ entspricht. Die Wechselstromverluste lassen sich also über $P_{g}$ und $k_{r}$ beeinflussen. Nun ergeben sich zwei unterschiedliche Wege, um die Größen der Gleichung (2-17) zu bestimmen.

Zum einen gibt es das sogenannte Teilleiterverfahren, das elektrische Leiter fiktiv in mehrere Teilleiter zerlegt. Anschließend werden für jeden Teilleiter einzeln die Ströme und Spannungen ermittelt. Bei diesem Verfahren wird auch von räumlicher Diskretisierung der Leiter gesprochen. Auf diesem Weg wird die Verlustleistung bei Wechselstrom $P_{w}$ direkt bestimmt. Im Prinzip wird bei diesem Berechnungsverfahren vorgegangen, wie es am Ende des Abschnitt 2.1.1 angedeutet wird. Ausgehend vom Teilleiterverfahren kann eine numerische Berechnung entwickelt werden, welche die Bestimmung der Stromverdrängung für beliebige Leiter- und Nutgeometrien ermöglicht. Dieser Berechnungsweg wird beispielsweise bei Müller u.a. [34] und Köhring [27] tiefergehend beleuchtet.

Neben dem Teilleiterverfahren existiert noch die analytisch geschlossene Berechnung der Stromverdrängung. Diese geht im Wesentlichen auf Sommerfeld [55], Field [11] und Emde [8] zurück. Der Berechnungsansatz basiert auf der maxwellschen und poyntingschen Sichtweise. Er kommt auf Gleichungen, mit denen auf Basis geometrischer und materialspezifischer Informationen direkt der Widerstandserhöhungsfaktor ermittelt werden kann. Dieser Ansatz wird beispielsweise bei Richter [50] und Müller u.a. [34] vorgestellt. Eine kompakte Herleitung der Zusammenhänge findet sich bei Emde [9]. Die analytisch geschlossene Berechnung der Stromverdrängung wird im Rahmen der vorliegenden Arbeit weiterführend betrachtetet.

Dem analytischen Berechnungsweg liegt ein recht rudimentäres Modell einer Nutteilung einer elektrischen Maschine zugrunde, wie es in Abbildung 2-1 gezeigt wird. 


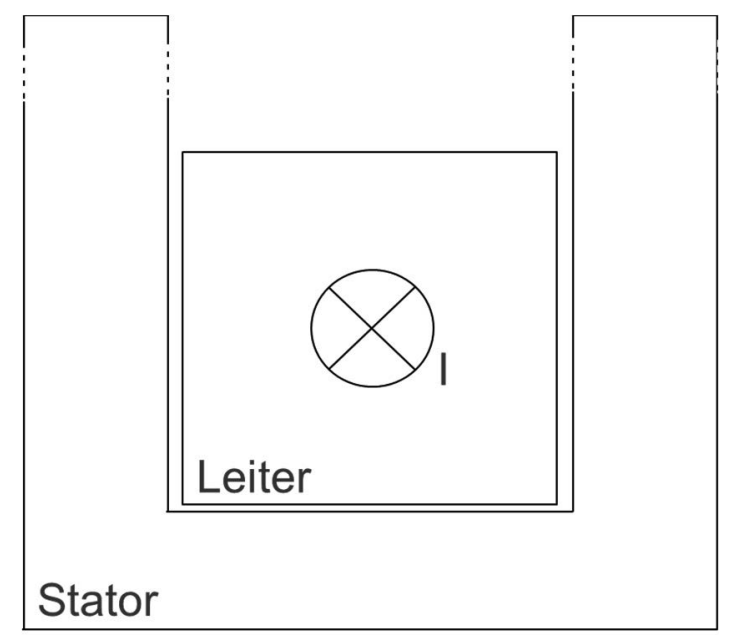

Abbildung 2-1: Der analytisch geschlossenenen Berechnung der Stromverdrängung zugrunde liegendes Modell einer Nutteilung

Es basiert auf einem rechteckigen Leiter, der an drei Seiten von ferromagnetischem Material umgeben ist. Bei Weiterentwicklungen wird von mehreren rechteckigen Leitern ausgegangen, die übereinander in der ferromagnetischen Nut liegen. Leiter und ferromagnetischer Teil werden unendlich lang angenommen. Die ferromagnetische Nut wird zusätzlich als unendlich tief angenommen, was durch die Strichelung in der Darstellung angedeutet wird. Innerhalb des ferromagnetischen Materials gibt es keine magnetischen Spannungsabfälle, da der magnetische Widerstand mit 0 angenommen wird. Bei mehreren Leitern in der Nut wird phasengleicher Strom in den Leitern angenommen. Strom fließt nur in die Bildebene und aus ihr heraus. Währenddessen bildet sich magnetischer Fluss außerhalb des ferromagnetischen Materials ausschließlich in horizontaler Richtung aus. Die Annahme beschreibt das sogenannte Nutquerfeld. In Kapitel 4 wird dargestellt, warum diese Annahme zunächst plausibel ist.

Auf Basis dieser Randbedingungen ergibt sich aus den Gleichungen (2-18), (2-19), (2-20), (2-21) und (2-22) für die Stromverdrängung

$$
k_{N p}=\varphi(\beta)+\frac{I_{u}\left(I_{u}+I_{p}\right)}{I_{p}^{2}} \psi(\beta) .
$$

Mit Gleichung (2-18) wird der Widerstandserhöhungsfaktor für die einzelnen, übereinanderliegenden Leiterlagen separat bestimmt. Die Gleichung gilt für das bereits erweiterte Ausgangsmodell, bei dem mehrere Leiter übereinander in der Nut liegen. Bei gleichen Strömen in den Leitern ergibt sich aus den in die Gleichung eingesetzten Strömen ein stromunabhängiger Faktor, der mit der Leiterlage variiert. $I_{u}$ meint den Strom der unterhalb des betrachteten Leiters in der Nut fließt. $I_{p}$ meint den Strom, der im betrachteten Leiter fließt. Bei

$$
\varphi(\beta)=\beta \frac{\sinh 2 \beta+\sin 2 \beta}{\cosh 2 \beta-\cos 2 \beta}
$$

und 


$$
\psi(\beta)=2 \beta \frac{\sinh \beta-\sin \beta}{\cosh \beta+\cos \beta}
$$

handelt es sich um Hilfsfunktionen. Ihr Verlauf ist in Abbildung 3-4 zu sehen.

$$
\beta=\alpha h_{l}
$$

beschreibt die sogenannte reduzierte Leiterhöhe massiver Leiter und bestimmt sich aus dem Reduktionsfaktor $a$, für den wiederum

$$
\alpha=\frac{1}{d_{e}}=\sqrt{\pi f \mu_{0} \kappa \frac{b_{l}}{b_{n}}}
$$

gilt. Legt man den vorgestellten Gleichungsapparat zugrunde, dann hängt der Wider-

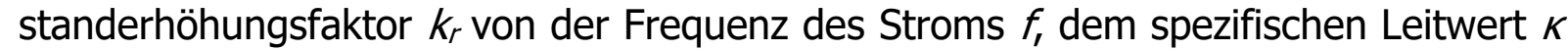

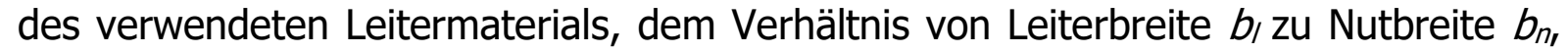
der Leiterhöhe $h$, und der Anzahl der übereinanderliegenden Leiter beziehungsweise Teilleiter ab.

Die Gleichungen haben sich in der Vergangenheit für den Auslegungsprozess elektrischer Maschinen bewährt. Dabei deutete bereits Field [11] eine wichtige Einschränkung an: Für die Gültigkeit der Gleichungen sind Zahnköpfe im Statorblechschnitt wichtig oder zumindest ein ausreichender Abstand der oben in der Nut liegenden Leiterlage zur Nutöffnung. Denn andernfalls streut das Luftspaltfeld so stark in die Nut ein, dass die Annahme eines reinen Nutquerfelds nicht mehr ausreichend genau ist. Dadurch verändern sich die Stromverdrängungsverhältnisse in den oberen Leitern. Im Abschnitt 1.3 wird auf weiterführende Ergänzungen in der Literatur zu den hier vorgestellten Grundgleichungen verwiesen. So wurden beispielsweise weitere Leiter- und Nutgeometrien untersucht.

\subsubsection{Begriffserklärungen und -abgrenzungen zur Stromverdrängung}

An dieser Stelle sollen Begriffe zur Stromverdrängung, die sich in der Literatur finden und auf unterschiedliche Modellvorstellungen zurückgehen, voneinander abgegrenzt werden. Im Schrifttum finden sich viele verschiedene Begrifflichkeiten, die aus Sicht des Autors der Abgrenzung und zumindest einer kurzen gedanklichen Einordnung bedürfen.

Der Begriff des Wirbelstroms und die maxwellsche Sichtweise wurden bereits in Abschnitt 2.1.2 erläutert. Ein weiterer Ausdruck, der sich in der Literatur findet, lautet Wechselstromwiderstand. Dieser geht auf die maxwellsche Sichtweise zurück. Danach hat ein Objekt, beispielsweise ein Leiter, einen Widerstand für Gleichstrom und einen anderen Widerstand für Wechselstrom. Der Gleichstromwiderstand hängt von den in Gleichung (2-2) gegebenen Größen ab. Der Wechselstromwiderstand hängt wiederum von weiteren geometrischen Beziehungen, dem umgebenden Material und der Frequenz des Wechselstroms ab. Die beiden Widerstände lassen sich ins Verhältnis setzen. Dieses Verhältnis wird 
Widerstandserhöhungsfaktor genannt und wird in Abschnitt 2.1.4 behandelt. Das Verhältnis gibt Auskunft über die Anfälligkeit eines Objekts für Stromverdrängung.

Aus dem Vergleich von Wechselstromwiderstand und Gleichstromwiderstand stammt auch der Begriff zusätzliche Kupferverluste, welche die Differenz zwischen der Leistung bei Wechselstrom und Gleichstrom meinen. Dahinter steckt der gedankliche Behelf, dass es die bekannten Gleichstromverluste nach dem ohmschen Gesetz gibt und dass durch den Einsatz von Wechselstrom nun noch zusätzliche Verluste entstehen.

Der Begriff Stromverdrängung passt nicht in die maxwellsche Sichtweise. Er geht auf die Modellvorstellung zurück, dass das magnetische Feld den ursprünglich gleichmäßig über den Leiterquerschnitt verteilten Strom verdrängt.

In der vorliegenden Arbeit wird zwischen den Begriffen gewechselt, da es aus Sicht des Autors vom jeweiligen Problem abhängt, welche Modellvorstellung gerade gut geeignet ist, um das Thema einfach und damit auch leicht verständlich zu formulieren. Der Leser sollte sich dabei immer der Zusammenhänge der jeweiligen Modellsichten bewusst sein.

\subsection{Permanenterregte Synchronmaschinen}

Inhalt dieses Abschnitts ist keine umfangreiche Beschreibung elektrischer Maschinen. Stattdessen werden für diese Arbeit relevante Eigenschaften elektrischer Maschinen kurz dargestellt. Dabei handelt es sich um Eigenschaften, die sich auf die Stromverdrängung auswirken oder mit ihr im Zusammenhang stehen.

Grundlegende und weiterführende Informationen zu elektrischen Maschinen finden sich beispielsweise bei Fischer [12] und Müller u.a. [33] [34].

Zunächst wird auf den Einsatz von elektrischen Traktionsantrieben in automobilen Hybridantrieben eingegangen (Abschnitt 2.2.1). Die Anwendung erfordert die Berücksichtigung des gesamten Kennfelds. Anschließend widmet sich Abschnitt 2.2.2 den verschiedenen Verlustarten. In Abschnitt 2.2.3 wird der Polradwinkel sowie sein Einfluss auf die Stromverdrängung geschildert. Dabei werden verschiedene Besonderheiten von Synchronmaschinen behandelt, die den Polradwinkel beeinflussen. Abschnitt 2.2.4 wendet sich unterschiedlichen Wickelschemata zu und zeigt, wie sich diese auf das magnetische Feld in der Nut auswirken können. Den Abschluss bildet Abschnitt 2.2.5 mit einer kurzen Ausführung zum Kupferfüllfaktor.

\subsubsection{Einsatz als Traktionsantrieb in automobilen Hybridantrieben}

Die in der vorliegenden Arbeit durchgeführten Untersuchungen wurden anhand einer elektrischen Maschine umgesetzt, die als Traktionsmotor in einem automobilen Hybridantrieb eingesetzt wird. Bei dieser Anwendung werden elektrische Maschinen im gesamten zur Verfügung stehenden Drehmoment-Drehzahl-Kennfeldbereich betrieben. In Abbildung 2-2 ist exemplarisch der motorische Kennfeldbereich gezeigt. 


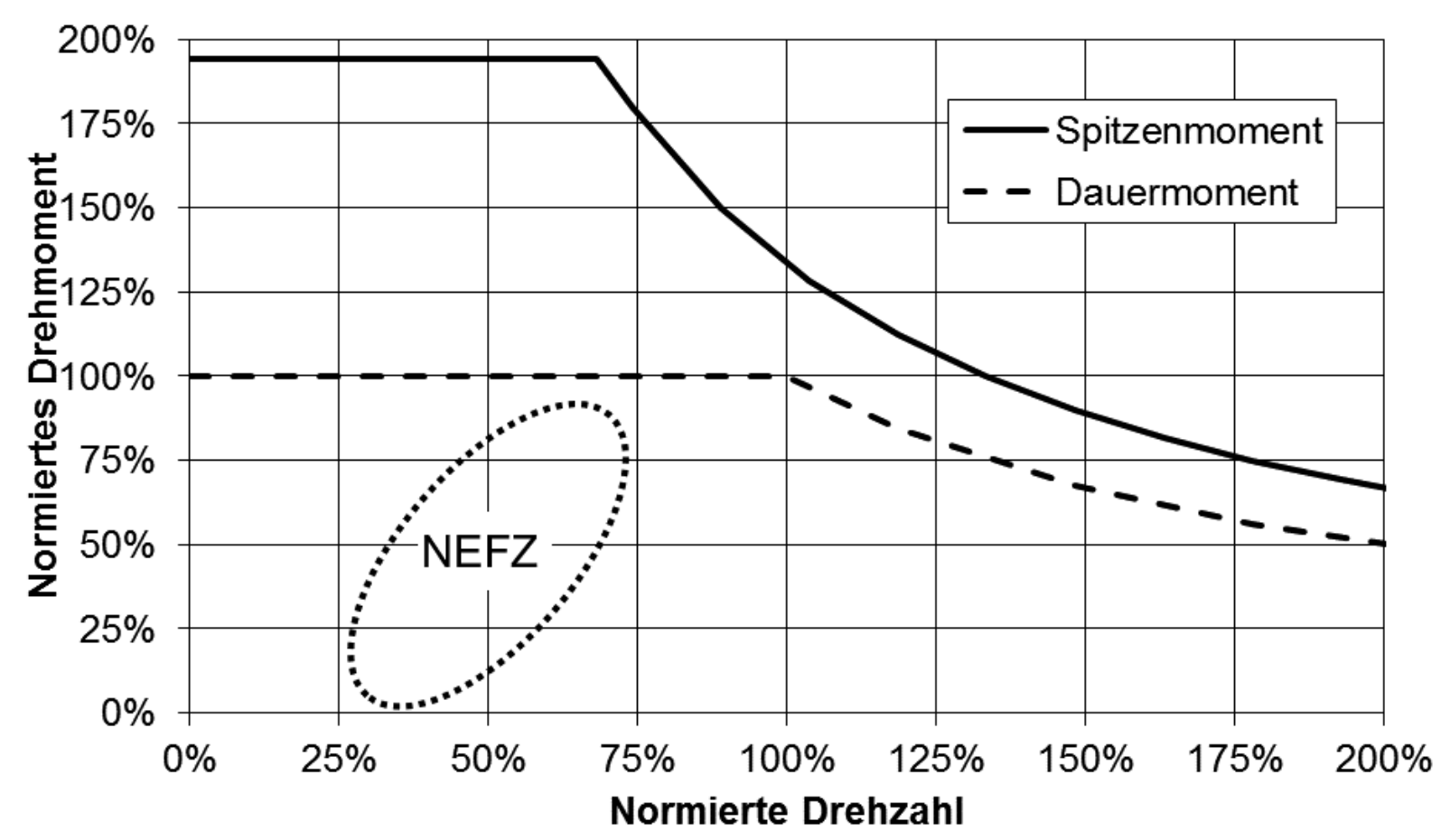

Abbildung 2-2: Beispiel eines Drehzahl-Drehmomenten-Kennfelds im motorischen Bereich mit Spitzenmoment- und Dauermoment-Kennlinie sowie dem NEFZ-relevanten Bereich

Die Spitzenmoment-Kennlinie zeigt das maximale Drehmoment, das die Maschine zur Verfügung stellen kann. Es kann durch unterschiedliche Parameter begrenzt sein, beispielsweise durch den maximalen Strom und die maximale Spannung, die durch die Batterie und die Leistungselektronik bereitgestellt werden können. Bis zur Spitzenmoment-Kennlinie kann die Maschine in jeder Kombination aus Drehmoment und Drehzahl betrieben werden. Diese Kombinationen werden als Betriebspunkte bezeichnet.

Zusätzlich ist in der Darstellung die Dauermoment-Kennlinie angegeben. Diese zeigt das Drehmoment, das die Maschine dauerhaft abgeben kann. Alle Betriebspunkte, die zwischen Spitzenmoment- und Dauermoment-Kennlinie liegen, können nur für eine begrenzte Zeit angefahren werden, da sie thermisch limitiert sind. In diesen Betriebspunkten entsteht aufgrund der Maschinenverluste und gegebenenfalls durch weitere äußere Einflüsse so viel Wärme, dass sie nicht in ausreichendem Maße abgeführt werden kann. Dadurch gelangt die Maschine nach einer bestimmten Zeit an ihre Temperaturgrenze.

Die gestrichelte Ellipse in Abbildung 2-2 markiert den Bereich, der für den neuen europäischen Fahrzyklus (NEFZ) besonders relevant ist. Dieser Zyklus stellte zum Zeitpunkt der Untersuchungen das Bewertungskriterium für den Wirkungsgrad der Maschine dar.

Jeder Betriebspunkt im Drehmoment-Drehzahl-Kennfeld weist seine eigenen Verluste auf. Diese beeinflussen zum einen den Wirkungsgrad der Maschine. Darüber hinaus beeinflussen die Verluste über das thermische Verhalten auch das Dauermoment, das die E-Maschine in der Lage ist bereitzustellen. Das in Abbildung 2-2 gezeigte Kennfeld stellt den motorischen Betrieb dar. Oftmals werden die gleichen elektrischen Maschinen in Hybridfahrzeugen auch generatorisch genutzt, um im Ver- 
zögerungsfall Energie zu rekuperieren. Weitere Informationen zum Einsatz elektrischer Maschinen in automobilen Hybridantrieben finden sich beispielsweise bei Hofmann [19].

\subsubsection{Verlustbetrachtung}

Wie im vorangegangenen Abschnitt erläutert wurde, weisen unterschiedliche Betriebspunkte verschiedene Wirkungsgrade und Verluste auf. Die auftretenden Verlustarten werden in diesem Abschnitt aufgegliedert.

In der vorliegenden Arbeit wird zwischen vier auftretenden Verlustgruppen unterschieden: Eisen-, Kupfer-, Magnet- und Reibungsverluste. Die Eisenverluste umfassen im Blechpaket auftretende Wirbelstrom- und Hystereseverluste. Die Kupferverluste beinhalten in den Spulen auftretende Gleichstrom- und Stromverdrängungsverluste. Die Magnetverluste meinen die Verluste in den Permanentmagneten, die durch Wirbelströme entstehen. Diese spielen in absoluten Werten oft nur eine untergeordnete Rolle. Da sie jedoch im Rotor auftreten, ist die durch sie entstehende Wärme schwerer abzuführen. Zu guter Letzt gibt es die Verlustkategorie Reibung, unter die beispielsweise Verluste durch die Lagerung des Rotors sowie Lüfterverluste fallen.

Bei der untersuchten elektrischen Maschine wird die Verlustenergie über die AuBenseite des Stators in einen Kühlmantel geführt. Dadurch kann die durch Eisenverluste im Statorblechpaket entstehende Wärme gut abgeführt werden. Schwieriger verhält es sich mit den Kupferverlusten. Bei diesen muss die Wärme erst von den Spulen aufs Blechpaket übertragen werden, um von dort in den Kühlmantel zu gelangen. Der Pfad von der Spule ins Blechpaket muss aus elektrotechnischer Sicht gut isoliert sein, was sich in den Regel negativ auf den thermischen Übergangswiderstand auswirkt. Dadurch lassen sich die Kupferverluste schlechter abführen als die Eisenverluste

\subsubsection{Einflüsse auf den Polradwinkel}

Die im Stator der Synchronmaschine liegende Drehstromwicklung erzeugt im Luftspalt der E-Maschine ein Drehfeld. Im stationären Betrieb werden die Pole des Rotors synchron vom umlaufenden Statordrehfeld mitgeschleppt. Dabei folgt der Rotor dem Statorfeld bei Belastung versetzt um den Polradwinkel $y=\angle\left(U_{S}, U_{P}\right)$. Wie aus Abschnitt 2.2.3.1 ersichtlich wird, ist ein definierter Polradwinkel notwendig, um das von der Maschine erzeugte Drehmoment zu maximieren. Der Winkel definiert auch, wie Stator- und Rotorfeld aufeinandertreffen. Das so entstehende resultierende Luftspaltfeld kann wiederum Einfluss auf die Stromverdrängung haben. Dieser Abschnitt gibt daher einen Einblick in die Eigenschaften elektrischer Maschinen, die den Polradwinkel beeinflussen.

\subsubsection{Reluktanz}

Das Drehmoment einer elektrischen Maschine ist nach Gerling [14] gegeben durch:

$$
\mathrm{T}=\frac{3 p}{\omega}\left(\frac{U_{S} U_{P}}{X_{d}} \sin (\gamma)+\frac{U_{S}^{2}}{2}\left(\frac{1}{X_{q}}-\frac{1}{X_{d}}\right) \sin (2 \gamma)\right)
$$


Es setzt sich aus zwei Anteilen zusammen: Der erste Teil beschreibt das synchrone Moment. Es wächst mit dem im Stator eingeprägten Strom und hängt vom Sinus des Polradwinkels ab. Der zweite Summand der Gleichung (2-23) stellt das Reluktanzmoment dar, das auf Basis der maxwellschen Kraft entsteht. Es hängt vom Sinus des doppelten Polradwinkels ab. Das Reluktanzmoment wächst mit dem Unterschied der Reaktanzen in d- und in q-Richtung. Die d-q-Achsenbetrachtung basiert auf rotorfesten Koordinaten, wie sie sich beispielsweise durch die Transformation nach Park [39] aus statorfesten Koordinaten ergeben.

Abbildung 2-3 zeigt exemplarisch die Überlagerung des synchronen- und des $\mathrm{Re}-$ luktanzmoments.

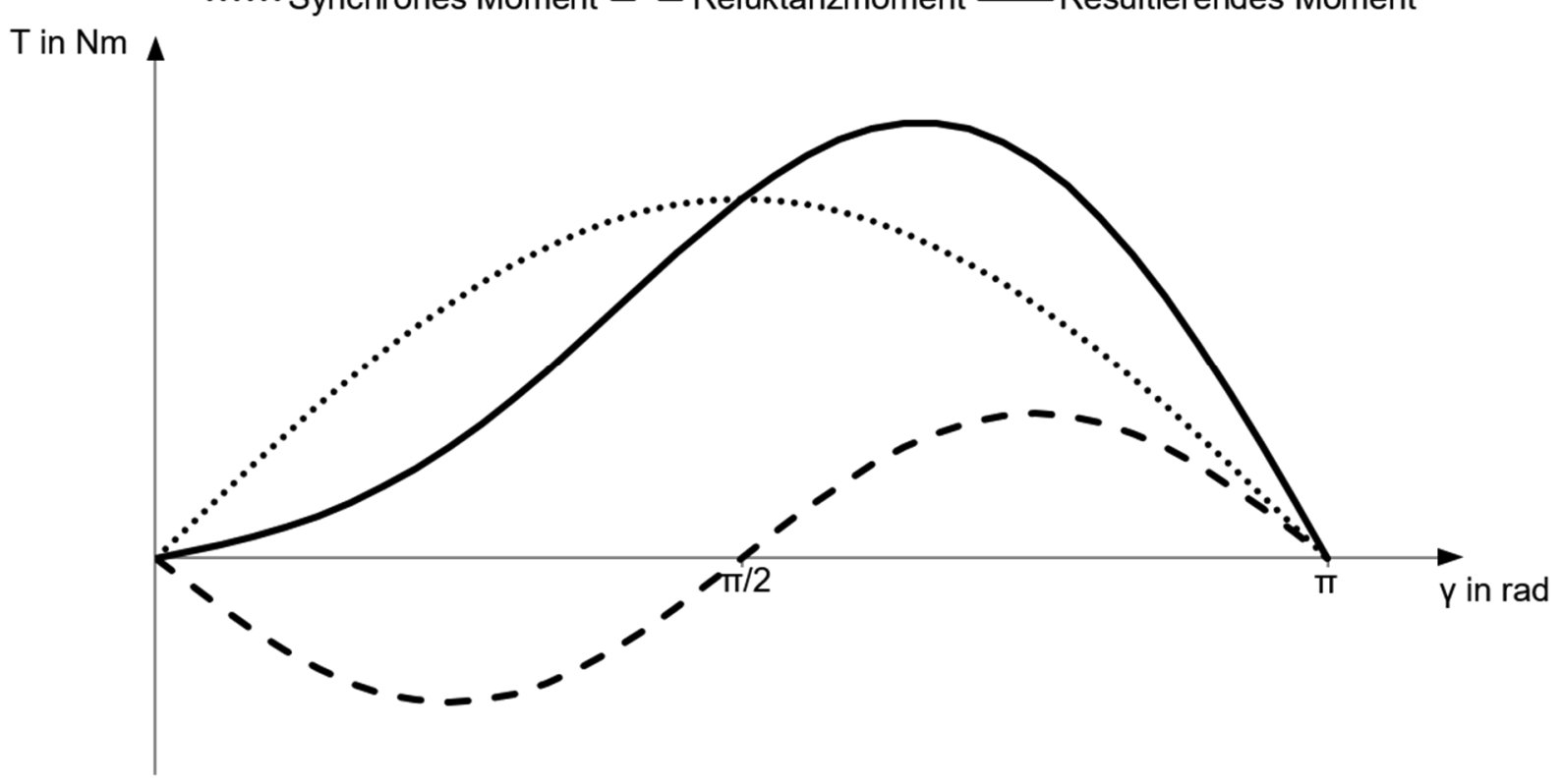

Abbildung 2-3: Entstehung des resultierenden Moments in Abhängikeit vom Polradwinkel bei Existenz eines Reluktanzmomentanteils

Die Größen sind dabei über den Polradwinkel aufgetragen. Der Reluktanzanteil wird oft gezielt genutzt. Dies ist durch den Betrieb am Umrichter möglich. Durch inn lässt sich der Stator abhängig von der Lage des Rotors so bestromen, dass das Statorfeld eine feste Relativlage zum Rotorfeld hat. Das ermöglicht eine Nutzung des Reluktanzmoments, mit der sich die Leistungsdichte der elektrischen Maschine steigern lässt [25]. Dabei muss auf ein sinnvolles Verhältnis der Reaktanzen in $d-$ und q-Richtung geachtet werden.

\subsubsection{Konstantfluss- und Feldschwächbereich}

Elektrische Maschinen sind in ihrer Performance durch den maximalen Leiterstrom

$$
\sqrt{I_{d}^{2}+I_{q}^{2}} \leq I_{\max }
$$

und die maximale Ausgangsspannung 


$$
\sqrt{U_{d}^{2}+U_{q}^{2}}=\omega \sqrt{\left(L_{d} I_{d}+\Psi_{P M}\right)^{2}+\left(L_{q} I_{q}\right)^{2}} \leq U_{\max }
$$

begrenzt. Darüber hinaus ist die Temperatur ein limitierender Faktor, der an dieser Stelle aber nicht betrachtet werden soll.

Die Spannungsgrenze spielt bei kleinen Frequenzen beziehungsweise Drehzahlen in der Regel keine Rolle. Aus Gleichung (2-25) ist ersichtlich, dass die Spannung mit der Kreisfrequenz $\omega$ ansteigt.

Im unteren Drehzahlbereich ist das maximale Drehmoment der Maschine durch den Strom begrenzt. Dieser Bereich wird als Grundstellbereich bezeichnet. Ab einer bestimmten Drehzahl wird die Spannungsgrenze erreicht. Dieser Punkt wird Eckpunkt genannt. Ab hier kann die Maschine ihr maximales Drehmoment nicht länger zur Verfügung stellen, um weiterhin Gleichung (2-25) zu genügen. Um dennoch höhere Drehzahlen realisieren zu können, muss das Permanentmagnetfeld des Rotors gezielt geschwächt werden. Dies geschieht, indem das Statorfeld dem Rotorfeld teilweise entgegengerichtet wird. Mit zunehmender Drehzahl muss das Rotorfeld stärker herabgesetzt werden. Dadurch nimmt das Drehmoment proportional mit der Kreisfrequenz ab, wie es in Abbildung 2-2 zu sehen ist. Zugleich bleibt die abgegebene Leistung der Maschine ab diesem Punkt konstant. Dieser Bereich wird Feldschwächbereich genannt. Um das Rotorfeld zu schwächen, ist eine Änderung des Polradwinkels erforderlich. Durch das Verfahren steigen aufgrund des zusätzlich notwendigen Feldschwächstroms auch die Kupferverluste.

\subsubsection{Schrägung}

Mit Schrägung ist gemeint, dass die Rotorpole und die Statornuten schräg zueinander verlaufen. Bei permanenterregten E-Maschinen wird dies oftmals erreicht, indem die Permanentmagnete im Rotor nicht in einer Linie hintereinander liegen. Stattdessen werden sie gestaffelt, das heißt in axialer Richtung stufig zueinander versetzt angeordnet. Dieser Fall ist exemplarisch in Abbildung 2-4 gezeigt.

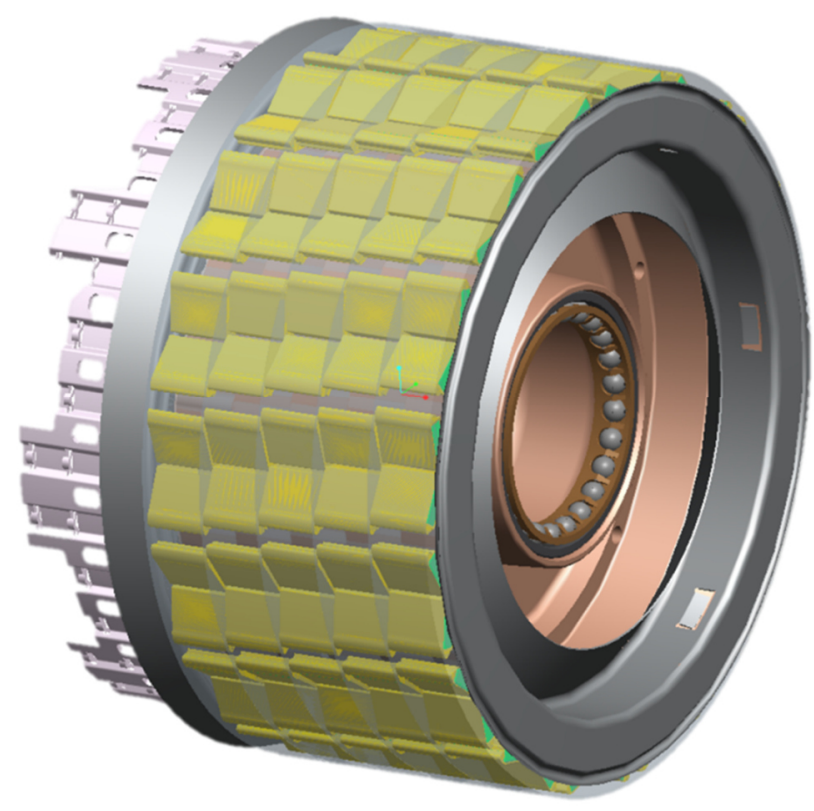

Abbildung 2-4: Gestaffelter Rotor mit transparentem Blechpaket 
Dabei ist das Rotorblechpaket transparent dargestellt, um die im Blechpaket eingebetteten Magnete sichtbar zu machen. Dadurch ist zu erkennen, dass die Magnete in jedem Segment leicht zueinander versetzt angelegt sind. Die Maßnahme ermöglicht es, magnetisch erzeugte Geräusche, starke Blechpaketschwingungen, Drehmomentschwankungen und das Rastmoment zu vermindern. Diese parasitären Effekte werden durch Oberwellen des Luftspaltfelds angeregt. Auf der anderen Seite verringert die Staffelung die Drehmomentbildung der Maschine, da nicht alle Segmente mit optimalem Polradwinkel betrieben werden können [34] [3] [14].

Bei der Staffelung handelt es sich um eine indirekte Beeinflussung des Polradwinkels. Für das mittlere Segment bleibt der Polradwinkel unverändert, für die gestaffelten Segmente verändert er sich jedoch.

\subsubsection{Wickelschema}

Auch das in elektrischen Maschinen angewendete Wickelschema hat Einfluss auf die Stromverdrängung in den Leitern. Um den Zusammenhang zu klären, muss die Spulenanordnung betrachtet werden: Eine Spule besteht aus zwei Spulenseiten. Dabei befindet sich jede Spulenseite in einer anderen Nut der elektrischen Maschine. Der Abstand in Nuten zwischen den beiden zusammengehörigen Spulenseiten wird Spulenweite oder Wickelschritt genannt. In elektrischen Maschinen werden oftmals Zweischichtwicklungen ausgeführt. Das bedeutet, dass zwei Spulenseiten von zwei unterschiedlichen Spulen in einer Nut liegen. Dadurch wird ein verkürzter Wickelschritt, die sogenannte Sehnung möglich. Durch Sehnung lassen sich parasitäre Oberwellen gezielt unterdrücken. Einen Sonderfall stellt im Zusammenhang mit der Zweischichtwicklung die Zahnspulenwicklung dar. Bei ihr befinden sich die Spulenseiten einer Spule in benachbarten Nuten. Die Spulen werden dabei üblicherweise um einen Zahn gewickelt. Dadurch liegen zwei Spulenseiten nebeneinander in einer Nut, während die Spulenseiten bei gewöhnlichen Zweischichtwicklungen übereinander angeordnet sind.

Gehören die beiden Spulenseiten einer Nut zu verschiedenen Strängen, so beeinflusst dies das magnetische Feld in der betrachteten Nut und dadurch die Stromverdrängung in den Leitern. Das in Abschnitt 2.1.4 verwendete Modell zur Berechnung der Stromverdrängung sieht nur phasengleichen Strom in den Leitern einer Nut vor. Eine ergänzende Betrachtung zu nebeneinander in der Nut angeordneten Spulenseiten mit unterschiedlicher Stromphase hat Mosebach [32] verfasst. $\mathrm{Er}$ kommt zu dem Ergebnis, dass das Nutquerfeld durch die Phasendifferenz der Ströme geschwächt wird. Die Schwächung sei umso höher, je größer die Phasendifferenz der Ströme in den beiden Spulenseiten ist. Die Folge sei eine verringerte Stromverdrängung. Im weiteren Verlauf der Arbeit werden die Erkenntnisse Mosebachs tiefergehend betrachtet. Weiterführende Informationen zu Wickelschemata finden sich beispielsweise bei Müller u.a. [33] und Gerling [14].

\subsubsection{Kupferfüllfaktor}

Nach Gleichung (2-1) und (2-2) lassen sich die Kupferverluste einer elektrischen Maschine reduzieren, indem der Leiterquerschnitt der Spulen erhöht wird. In manchen E-Maschinen besteht jedoch ein Bauraumproblem, da der Nutraum, in den die Spulen eingelegt werden, begrenzt ist. Dadurch kann der Leiterquerschnitt nicht beliebig erhöht werden. Hier gibt es allerdings Optimierungsreserven, denn das Potenzial bei 
der Nutzung des Nutraums wird bisher noch nicht ausgeschöpft. Der Kupferfüllfaktor $\varphi_{c u}$ ist nach Bickel u.a. [2] definiert als:

$$
\varphi_{c u}=\frac{z_{n} A_{l}}{A_{n}}
$$

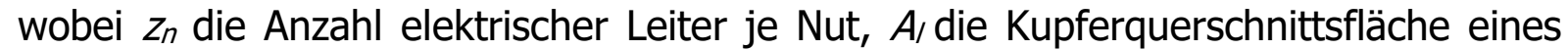
Leiters und $A_{n}$ die Querschnittfläche der Nut meint. Üblicherweise werden in Großserienprozessen nach Gleichung (2-26) Kupferfüllfaktoren von höchstens 55\% erreicht, bezogen auf die gesamte Nutfläche. Die restlichen $45 \%$ werden unter anderem für die Leiterisolation und die Nutwandisolation benötigt. Ein nicht unbedeutender Anteil ist aber auch auf Hohlräume zurückzuführen, die keinen Nutzen erfüllen. Durch eine Abstimmung von Nut- und Leiterquerschnittsgeometrie gibt es hier signifikantes Verbesserungspotential. Prototypisch konnte dies bereits mit Hilfe gegossener Spulen, wie sie in Abbildung 3-1 und Abbildung 3-3 zu sehen sind, genutzt werden. Mit diesen konnte ein Kupferfüllfaktor von rund 75\% erreicht werden [24] [23] [16] [17]. Eine Steigerung des Kupferfüllfaktors ist auch für eine Großserie denkbar. Dies ist nach den Gleichungen (2-1), (2-2) und (2-17) gleichbedeutend mit einer Reduzierung der Kupferverluste, sofern der Widerstandserhöhungsfaktor durch den Anstieg des Kupferfüllfaktors nicht zu stark beeinflusst wird.

\subsection{Finite-Elemente-Berechnung}

Bei der Finite-Elemente-Methode (FEM) handelt es sich um ein numerisches Verfahren. Mit ihm lassen sich die maxwellschen Gleichungen für komplexe Maschinengeometrien lösen. Dabei wird eine erstellte Maschinengeometrie mit einem Netz, dem sogenannten Mesh, überzogen. Dies ist exemplarisch in Abbildung 2-5 dargestellt. In jedem Netzelement wird das Vorliegen eines homogenen Magnetfelds unterstellt. 


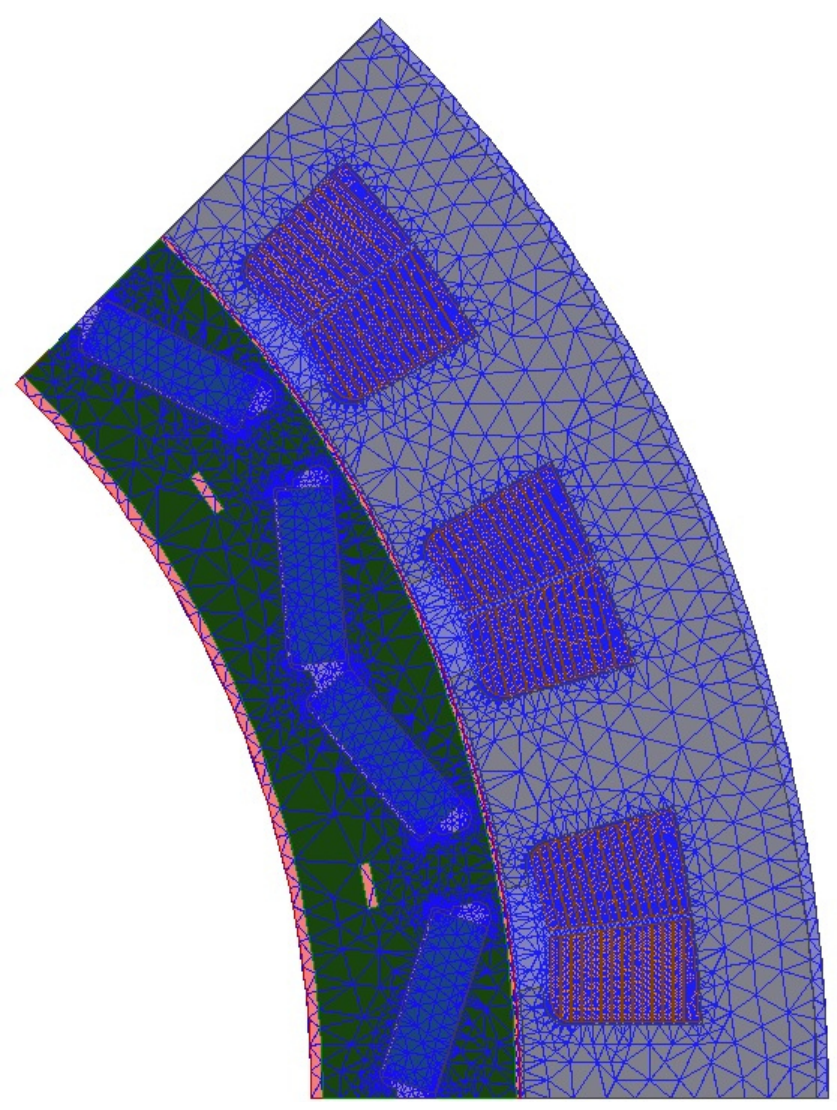

Abbildung 2-5: Mesh des 2D-Finite-Elemente-Modells der E-Maschine mit gegossenen Spulen

Üblicherweise besteht das Mesh aus dreieckigen, rechteckigen oder trapezförmigen Elementen. Durch die Zerlegung in vereinfachte Elemente können aufwendige Geometrien und Feldprobleme behandelt werden. Es lassen sich sowohl Lösungen für 2D- als auch für 3D-Geometrien bestimmen. Die Genauigkeit des Verfahrens hängt auch von der Größe und der Form der Netzelemente ab. Für die Untersuchung von Stromverdrängungseffekten in den Leitern einer elektrischen Maschine ist es erforderlich, jeden Leiter einzeln zu modellieren. Dies ist für den regulären Auslegungsprozess zu aufwendig. In der vorliegenden Arbeit wurde Maxwell 15.0 von der Firma Ansys für die Finite-Elemente-Berechnung verwendet. Dabei handelt es sich um ein kommerzielles Programm für elektromagnetische Berechnungen. Es bietet einen Modeller zur Erstellung von elektrischen Maschinenmodellen, ist aber auch in der Lage, CAD-Daten zu verarbeiten. Weiterführende Informationen zur Verwendung des Verfahrens der Finiten-Elemente in Bezug auf elektrische Maschinen finden sich beispielsweise bei Müller u.a. [34].

Im Rahmen der Arbeit werden zwei unterschiedliche Finite-Elemente-Modelle für Untersuchungen verwendet: zum einen das Modell einer elektrischen Maschine mit gegossenen Spulen, das in Abschnitt 3.3 gezeigt wird, und zum anderen ein Modell, das sich an der vereinfachten Geometrie für das in Abschnitt 2.1.4 beschriebene analytische Verfahren orientiert. Dieses Modell wird im Folgenden Grundmodell genannt und wird in Abschnitt 4.2 vorgestellt. Das Grundmodell gibt es in zwei Ausführungen des magnetischen Kreises: mit und ohne magnetischen Rückschluss. Des Weiteren werden beim Grundmodell die Leiteranordnungen und -eigenschaften sowie die Blecheigenschaften für verschiedene Versuche variiert. 


\section{Vergleich des Widerstandserhöhungsfaktors}

In diesem Kapitel wird ein Vergleich von auf verschiedene Weise ermittelten Werten des Widerstandserhöhungsfaktors vorgenommen, um zu überprüfen, ob die Ermittlungsverfahren für die untersuchte Maschine zuverlässig sind. Dazu wird der Widerstandserhöhungsfaktor auf drei Arten bestimmt: mittels analytischer Berechnung, durch die Finite-Elemente-Methode sowie durch Messung am Prüfstand.

In Abschnitt 3.1 wird die für den Vergleich verwendete Maschine erläutert. Anschließend wird auf die analytische Bestimmung (Abschnitt 3.2), die Ermittlung mittels Finite-Elemente-Methode (Abschnitt 3.3) und den Prüfstandsbestimmungsweg (Abschnitt 3.4) eingegangen. Abschnitt 3.5 stellt die Ergebnisse des Vergleichs vor. Abschließend wird in Abschnitt 3.6 eine erste Einordnung und Interpretation der Ergebnisse vorgenommen.

\subsection{Für den Vergleich verwendete E-Maschine}

Für den Vergleich wurde eine Versuchsmaschine verwendet, die auf dem Fahrmotor des Volkswagen Golf GTE basiert.

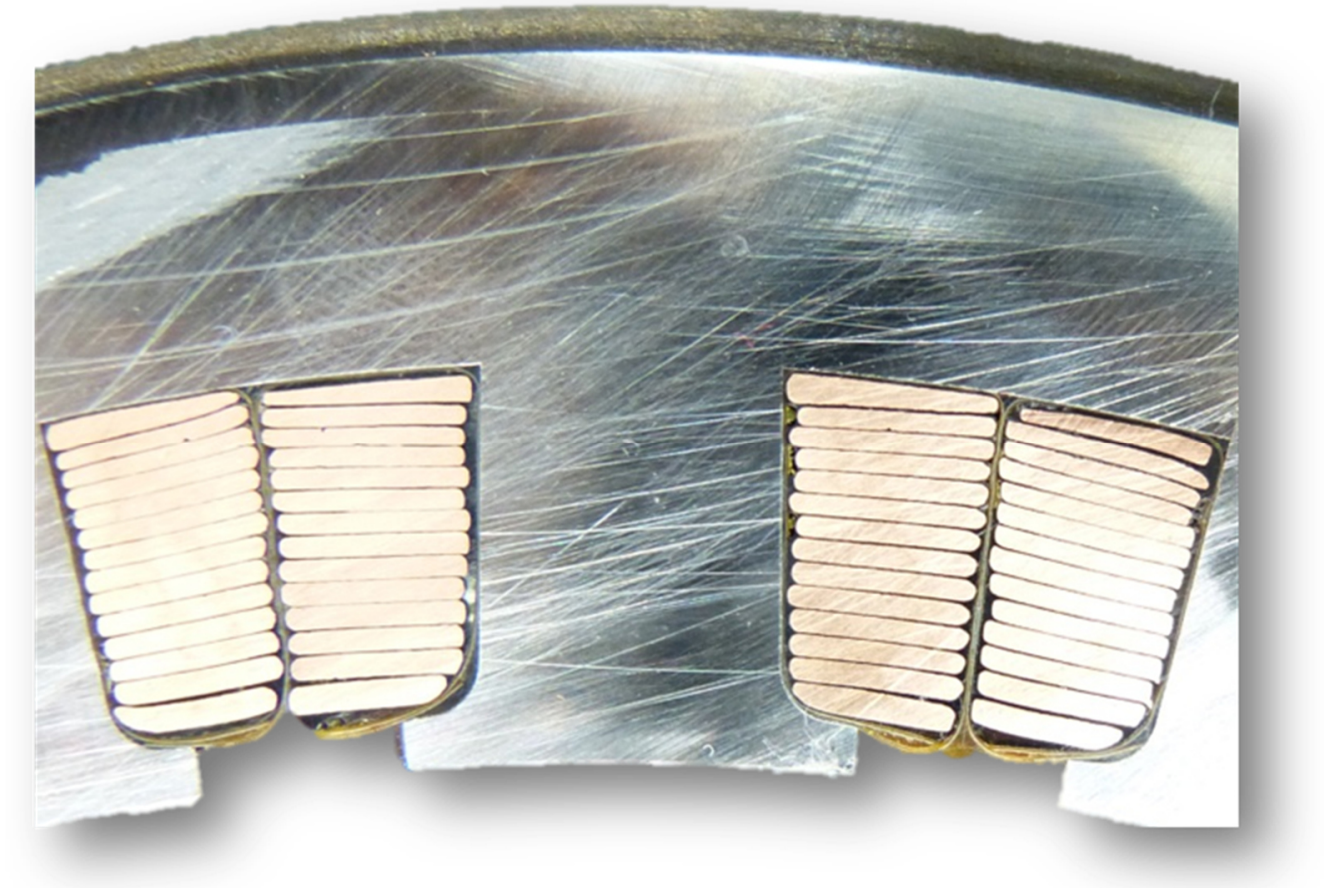

Abbildung 3-1: Schnitt durch den Stator

Wesentliche Änderungen gegenüber der Basisauslegung sind dabei die Verwendung von gegossenen Spulen [24] [23] [17] [16] für einen gesteigerten Kupferfüllfaktor und Zahnköpfe, die im ursprünglichen Blechschnitt nicht enthalten sind. Die Auslegung zielt dabei auf geringe Kupferverluste ab. Durch den hohen Kupferfüllfaktor werden die Kupferverluste bei homogen verteiltem Strom reduziert, und durch flache, breite Leiter wird der Widerstandserhöhungsfaktor $k_{r}$ gemäß Gleichung (2-18) begrenzt. Die Zahnköpfe wurden zusätzlich in den Statorblechschnitt eingebracht, um 
das Eindringen des Luftspaltfelds in die Nuten zu verringern. Durch diese Maßnahme werden Stromverdrängungsverluste durch das Hauptfeld reduziert.

Die genannten Optimierungsmaßnahmen sind aus Abbildung 3-1 ersichtlich. Dort ist ein Schnitt durch den Stator gezeigt. In Abbildung 3-2 ist das in testfähigem Zustand aufgebaute Hybridmodul illustriert, in das die elektrische Maschine integriert ist.

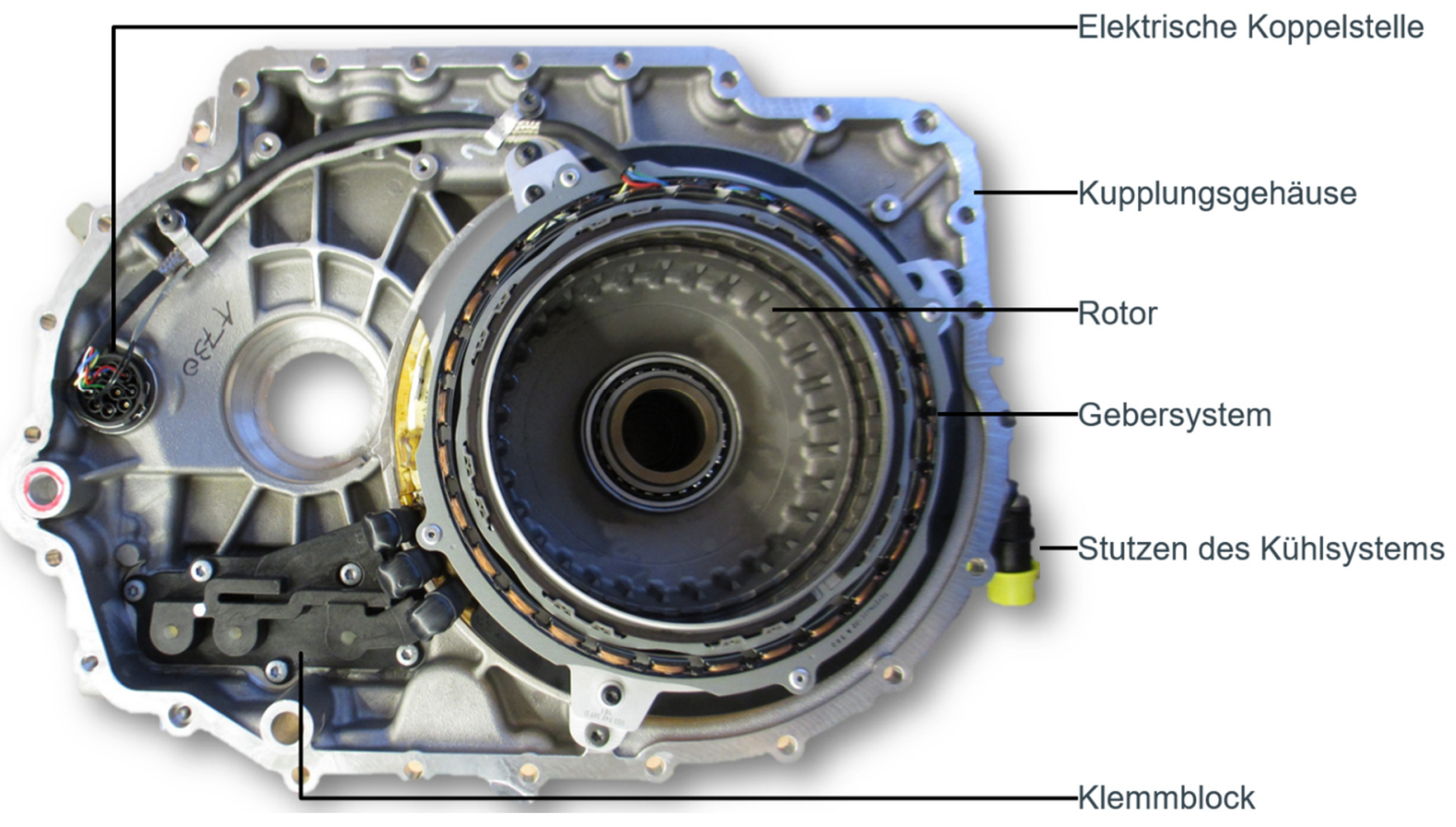

Abbildung 3-2: Hybridmodul

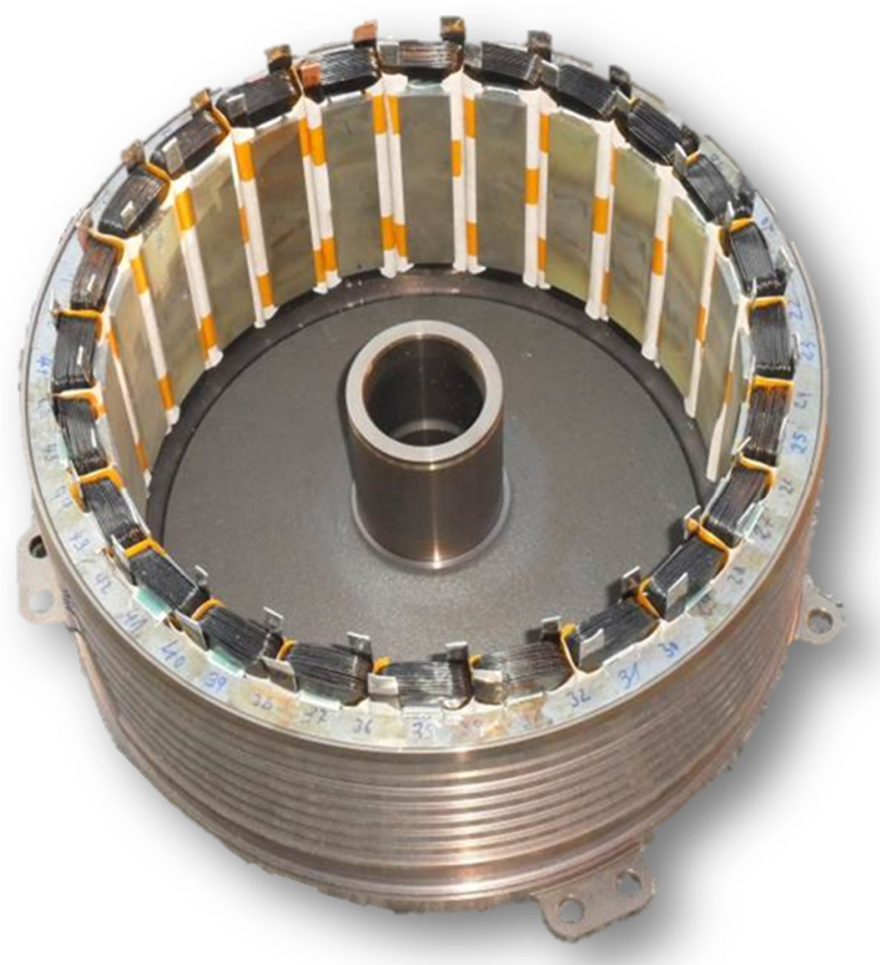

Abbildung 3-3: Stator im Kühlmantel 
Ein besserer Überblick über den Rotor ergibt sich aus Abbildung 2-4, während Abbildung 3-3 einen Eindruck des Stators vermittelt. Der Stator ist noch ohne Schaltung der Spulen gezeigt. Es ist gut zu erkennen, dass Zahnspulen verwendet wurden.

Die Eckdaten der Maschine sind in Tabelle 1 aufgeführt.

\begin{tabular}{c|c} 
Parameter & Werte \\
\hline Bemessungsspannung & $210 \mathrm{~V}$ \\
Bemessungs- / max. Strom & $235 \mathrm{~A} / 420 \mathrm{~A}$ \\
Bemessungs- / max. Leistung & $45 \mathrm{~kW} / 80 \mathrm{~kW}$ \\
Bemessungs- / max. Drehzahl & $2500 \mathrm{~min}^{-1} / 7000 \mathrm{~min}^{-1}$ \\
Bemessungs- / max. Drehmoment & $170 \mathrm{Nm} / 330 \mathrm{Nm}$ \\
Polpaarzahl & 8 \\
Nutzahl & 24 \\
Nutbreite unten & $19,7 \mathrm{~mm}$ \\
Nutbreite oben & $15,09 \mathrm{~mm}$ \\
Höhe der Nut ohne Zahnköpfe & $14,07 \mathrm{~mm}$ \\
Windungszahl je Spule & 14 \\
Parallelgeschaltete Teilleiter je Leiter & 1 \\
Lochzahl der Wicklung & $1 / 2$ \\
Paketlänge & $95 \mathrm{~mm}$ \\
Mittlerer Leiterquerschnitt blank & $6,88 \mathrm{~mm}$ \\
Mittlere Leiterhöhe blank & $0,86 \mathrm{~mm}$ \\
Mittlere Leiterbreite blank & $7,91 \mathrm{~mm}$
\end{tabular}

Tabelle 1: Maschineneigenschaften

\subsection{Analytische Bestimmung}

Für den Abgleich wird das in Abschnitt 2.1.4 beschriebene analytische Verfahren auf die untersuchte E-Maschine angewendet. Dabei wird die Stromverdrängung mit Hilfe der notwendigen geometrischen Daten, die der Tabelle 1 zu entnehmen sind, für mehrere betriebsrelevante Frequenzen bestimmt.

Aufgrund der rechteckigen Leiterquerschnittsgeometrie der E-Maschine mit gegossenen Spulen gestaltet sich die Ermittlung des mittleren Widerstandserhöhungsfaktors $k_{r}$ relativ einfach. Dennoch gibt es einige Aspekte, die beachtet werden müssen. So besitzen die einzelnen Leiterlagen der untersuchten E-Maschine unterschiedliche Leiterhöhen. Dies lässt sich in Gleichung (2-21) berücksichtigen. Des Weiteren gilt der mittels Gleichung (2-18) ermittelte Widerstandserhöhungsfaktor $k_{r}$ nur für die Bereiche der Spule, die in der Nut liegen. In den Wickelköpfen herrschen andere Verhältnisse. Da die Wickelkopfabschnitte bei Zahnspulen relativ kurz sind 
und damit einen geringen Anteil der Spule ausmachen, wird der Widerstandserhöhungsfaktor in diesen Abschnitten vereinfacht mit 1 angenommen. Die Verrechnung der beiden Abschnittsarten geschieht über eine gewichtete Addition, wie sie bei Müller u.a. [34] vorgestellt wird.

Ein weiterer zu berücksichtigender Aspekt ist die Temperatur der Wicklung. Da die Prüfstandsversuche bei einer Wicklungstemperatur von $105^{\circ} \mathrm{C}$ durchgeführt wurden, ist auch bei der analytischen Betrachtung eine Umrechnung auf diese Temperatur nötig. Dies geschieht mit Hilfe der Gleichung [18]

$$
R(\vartheta)=R\left(\vartheta_{0}\right)\left(1+\alpha_{\vartheta 0}\left(\vartheta-\vartheta_{0}\right)\right)
$$

Weiterhin ist zu beachten, dass sich das analytische Modell auf offene Nuten bezieht. Doch Rogowski [51] zeigt, dass es sich auch auf durch Zahnköpfe halb geschlossene Nuten anwenden lässt. Auch erfordert die konische Nutform Berücksichtigung, da das analytische Modell aus Abschnitt 2.1.4 von parallelflankigen Nuten ausgeht. Die Auswirkungen lassen sich mit Hilfe von Laibles Erkenntnissen [29] einbeziehen.

Eine Besonderheit der verwendeten gegossenen Spulen kann an dieser Stelle bereits vorweggenommen werden. Da die Frequenz nach Gleichung (2-22) radiziert in den Reduktionsfaktor a eingeht, würde man beim Abtragen des Widerstandserhöhungsfaktors $k_{r}$ über die Frequenz den Verlauf einer Wurzelfunktion erwarten. Der Reduktionsfaktor $a$ geht in die reduzierte Leiterhöhe $\beta$ linear ein. Jedoch beeinflusst der Wert der reduzierten Leiterhöhe das Verhalten, mit dem die reduzierte Leiterhöhe in die Hilfsfunktionen $\varphi(\beta)$ und $\psi(\beta)$ eingeht. Bei hohen Werten der reduzierten Leiterhöhe geht diese, wie es aus Abbildung 3-4 ersichtlich ist, näherungsweise linear in die Hilfsfunktionen $\varphi(\beta)$ und $\psi(\beta)$ ein.

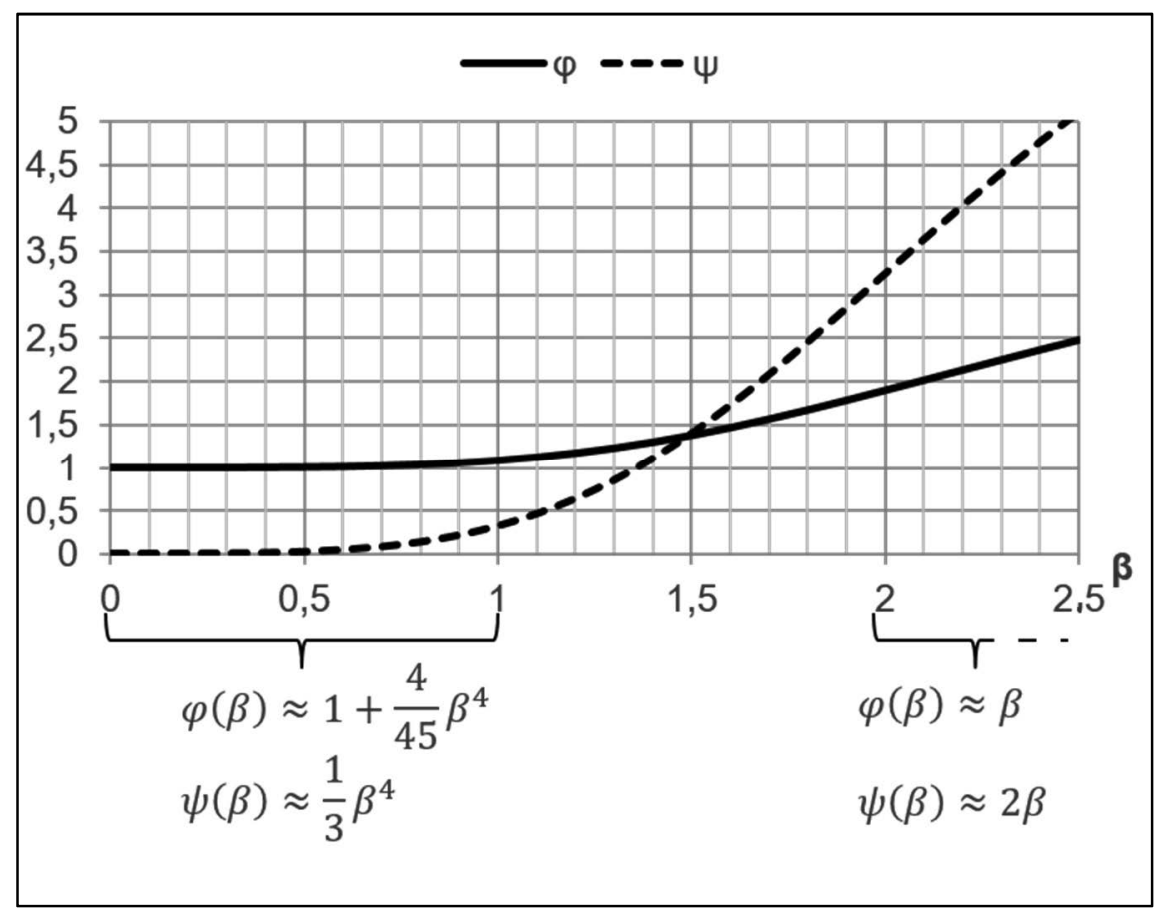

Abbildung 3-4: Verlauf der Hilfsfunktionen $\varphi(\beta)$ und $\psi(\beta)$ 
Dadurch erhält man tatsächlich den Verlauf einer Wurzelfunktion für den über der Frequenz aufgetragenen Widerstandserhöhungsfaktor $k_{r}$. Dagegen führen geringe Werte der reduzierten Leiterhöhe dazu, dass sie näherungsweise mit der vierten Potenz in die Hilfsfunktionen eingeht. Dadurch erhält man einen quadratischen Verlauf des Widerstandserhöhungsfaktors über der Frequenz. Die auf einen möglichst geringen Widerstandserhöhungsfaktor optimierten gegossenen Spulen weisen eine sehr geringe Leiterhöhe auf. Die reduzierte Leiterhöhe liegt bei niedrigen und mittleren Frequenzen in dem Bereich, in dem sie mit der vierten Potenz in die Hilfsfunktionen eingeht. Dadurch hat der Verlauf des Widerstandserhöhungsfaktors über der Frequenz für die gegossenen Spulen zunächst einen quadratischen Anstieg.

\subsection{Bestimmung mittels Finite-Elemente-Methode}

Die Geometrie des Modells wurde mit Hilfe von CAD-Daten erstellt. Wichtige Maße und Eigenschaften der E-Maschine finden sich in Tabelle 1. Die Materialeigenschaften der Permanentmagnete und des Elektroblechs basieren auf Angaben der jeweiligen Hersteller C.D. Wälzholz und Vacuumschmelze. Zusätzlich wurde für das Blechpaket ein Stapelfaktor von $96 \%$ angenommen. Für die Kupfereigenschaften wurden Einzelmessungen der gegossenen Spulen herangezogen und mit der analytischen Widerstandsbestimmung abgeglichen, wie Anhang A zu entnehmen ist. Die Nuten werden durch die axiale Länge des Modells berücksichtigt. Wie in Abbildung 3-5 zu erkennen ist, wurden die einzelnen Leiter separat modelliert.

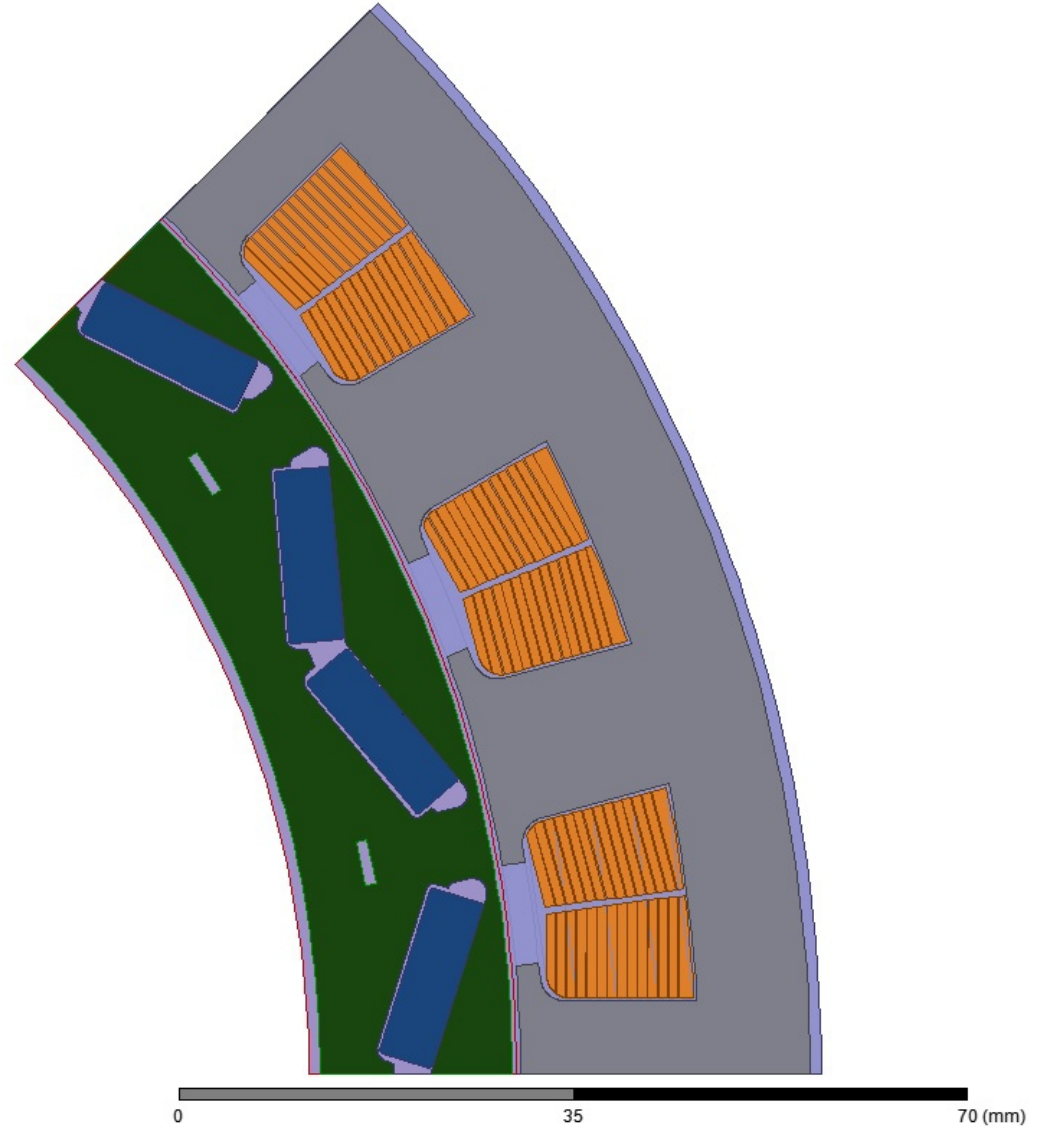

Abbildung 3-5: Geometrie des Modells der elektrischen Maschine mit gegossenen Spulen 
Da sämtliche Leiterlagen einer Spule in Reihe geschaltet sind, ist es möglich, einen festen Strom einzuprägen. Das Modell wird mit 22.597 Elementen nach Abbildung 2-5 vernetzt. Die hohe Anzahl der Elemente ist notwendig, um eine ausreichend genaue Bestimmung der Verhältnisse in den einzelnen Leitern zu gewährleisten. Das Feldproblem kann frei wählbar für verschiedene Frequenzen gelöst werden. Bei maximaler Betriebsfrequenz des Stroms von $933 \mathrm{~Hz}$ und einer Kupfertemperatur von $105^{\circ} \mathrm{C}$ liegt die Eindringtiefe in die elektrischen Leiter bei $2,5 \mathrm{~mm}$. Somit ist die Eindringtiefe für die Güte der Berechnung nicht ausschlaggebend. Kritischer verhält es sich mit der Leiterhöhe. Diese liegt im Mittel lediglich bei 0,86 mm. Daher wird in den Leitern eine maximale Seitenlänge der Mesh-Dreiecke von 0,3 mm gewählt. So liegt die Elementhöhe zwischen 0,26 und 0,3 mm, und es kann gewährleistet werden, dass bei der Berechnung drei Mesh-Dreiecke übereinander liegen.

Für die Bestimmung der Stromverdrängung wird die Berechnung des betrachteten Betriebspunkts zweifach durchgeführt. Bei der ersten Berechnung wird über die Einstelloptionen des verwendeten Finite-Elemente-Berechnungsprogramms die Rückwirkung magnetischer Felder auf die Stromdichteverteilung vernachlässigt. Dadurch ergibt sich eine homogene Stromdichteverteilung im Leiter. Mit Hilfe der Gleichung

$$
P_{c u}=\iiint_{V} \rho J^{2} d V
$$

wird die Verlustleistung berechnet und man erhält die Kupferverluste bei homogen verteiltem Strom. Für das Volumen werden die modellierte Querschnittsfläche der Leiter sowie die Paketlänge verwendet. Bei der zweiten Simulation des Betriebspunkts wird die Rückwirkung des magnetischen Felds auf die Stromdichteverteilung berücksichtigt. Dadurch ergeben sich die Verhältnisse mit Stromverdrängung $P_{w}$. Aus den so gewonnen Größen kann der Widerstandserhöhungsfaktor gemäß Gleichung (2-17) ermittelt werden.

Die Ermittlung des Widerstandserhöhungsfaktors $k_{r}$ mit Hilfe der Finite-ElementeBerechnung erfolgt entlang der in Abbildung 2-2 gezeigten Spitzenmoment-Kennlinie. Dafür werden die gleichen Leiterströme wie bei der Messreihe am Prüfstand eingespeist und auch der gleiche Polradwinkel realisiert. Die Prüfstandsmessung wird in Abschnitt 3.4 erläutert.

Wie zu erwarten ist, stimmt der Gleichstromwiderstand der Leiter bei der Berechnung auf analytischem Weg und über Finite-Elemente-Rechnung gut überein, wie aus Anhang A ersichtlich wird. Da die Finite-Elemente-Berechnungen auf Basis von 2D-Modellen durchgeführt werden, ist eine zusätzliche Berücksichtigung der Wickelkopfabschnitte notwendig. Daher wird die in den Wickelköpfen umgesetzte Leistung auf analytischem Weg berechnet und hinzuaddiert. Dabei werden, ebenso wie bei der analytischen Berechnung, auftretende Stromverdrängungsverluste im Wickelkopf vernachlässigt. Die Stromverdrängung im Wickelkopf spielt nur eine untergeordnete Rolle im Vergleich zur auftretenden Stromverdrängung in den Nuten, wie sich aus den Gleichungen (2-14) und (2-15) abschätzen lässt.

Ein weiterer zu berücksichtigender Aspekt bei der Finite-Elemente-Berechnung ist die Temperatur der Wicklung. Da die Prüfstandsversuche bei einer Wicklungstemperatur von $105{ }^{\circ} \mathrm{C}$ durchgeführt werden, basieren auch die Finite-Elemente- 
Berechnungen auf einer Kupfertemperatur von $105^{\circ} \mathrm{C}$. Für den analytisch berücksichtigten Wickelkopf ist eine Umrechnung mit Hilfe von Gleichung (3-1) notwendig.

\subsection{Messungen auf dem Prüfstand}

Für die Messungen am Prüfstand wird die in Abschnitt 3.1 beschriebene Versuchsmaschine verwendet. Dazu wird sie bei festen Betriebspunkten, also bei konstanten Drehzahlen und Drehmomenten, betrieben. Die Betriebspunkte liegen entlang der in Abbildung 2-2 dargestellten Spitzenmoment-Kennlinie. Sie werden über eine Lastmaschine eingestellt.

Der Prüfstandsaufbau entspricht der Darstellung in Abbildung 3-6.

Wechselrichter

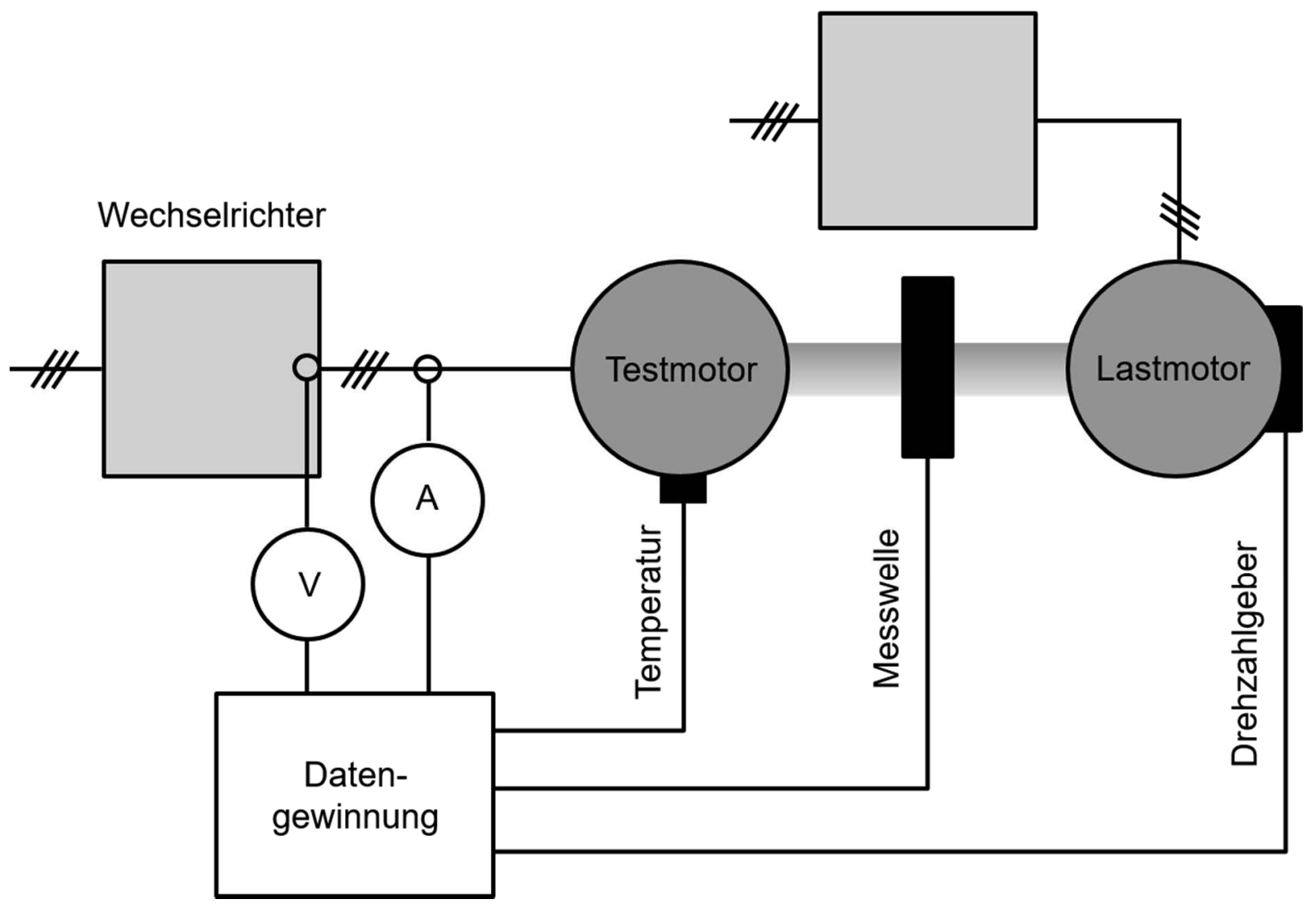

Abbildung 3-6: Prüfstandsaufbau

Dabei wird die Drehzahl mit Hilfe eines Drehzahlgebers Heidenhain ERM220 bestimmt. Die Wechselrichter stammen aus der SKAI-Baureihe der Firma Semikron. Die Spannung wird direkt am Wechselrichter abgegriffen, während der Strom mit Hilfe eines IT 700-Stromwandlers der Firma LEM gemessen wird. Das Drehmoment wird von einem T12-Drehmomentaufnehmer der Firma HBM erfasst. Die zentrale Datenverarbeitung erfolgt mit Hilfe eines Leistungsanalysators Yokogawa WT 3000. Die Leiterspannungen, Leiterströme, Drehmomente, Drehzahlen und Rotorlagedaten werden mit einer Rate von 200 kHz erfasst. Die Messroutine ist dabei vollständig automatisiert. Die mechanische Leistung

$$
P_{\text {mech }}=2 \pi T n
$$


wird aus den erfassten Messwerten des Drehmoments und der Drehzahl bestimmt. Die elektrische Leistung

$$
P_{e l}=\frac{1}{t_{T}} \int_{0}^{t_{T}} u(t) i(t) d t
$$

wird aus den gemessenen Momentanwerten des Stroms und der Spannung gemittelt.

Für die Bestimmung des Widerstandserhöhungsfaktors $k_{r}$ gemäß Gleichung (2-17) wird die Verlustleistung in der Wicklung bei Gleichstrom $P_{c u, g}$ und bei Wechselstrom $P_{c u}$ benötigt. Die Verlustleistung bei Gleichstrom lässt sich durch eine Widerstandsmessung auch ohne Prüfstand leicht ermitteln. Für die Verlustleistung in den Leitern bei Wechselstrom ist eine Messung im Betrieb erforderlich, denn es sollen sämtliche Einflüsse berücksichtigt werden, die die Stromverteilung im Leiter beeinflussen.

Abbildung 3-7 zeigt exemplarisch am Prüfstand ermittelte Daten. Es handelt sich um die Ermittlung des sogenannten 60 -Sekunden-Moments bei rund $310 \mathrm{~Hz}$. Beim 60-Sekunden-Moment wird das Drehmoment ermittelt, das die elektrische Maschine konstant für 60 Sekunden abgeben kann. Dieses ist thermisch limitiert. Daher ist in der Darstellung neben der elektrischen und der mechanischen Leistung auch die Wickelkopftemperatur aufgetragen. Sie erreicht nach 60 Sekunden ihren Grenzwert.

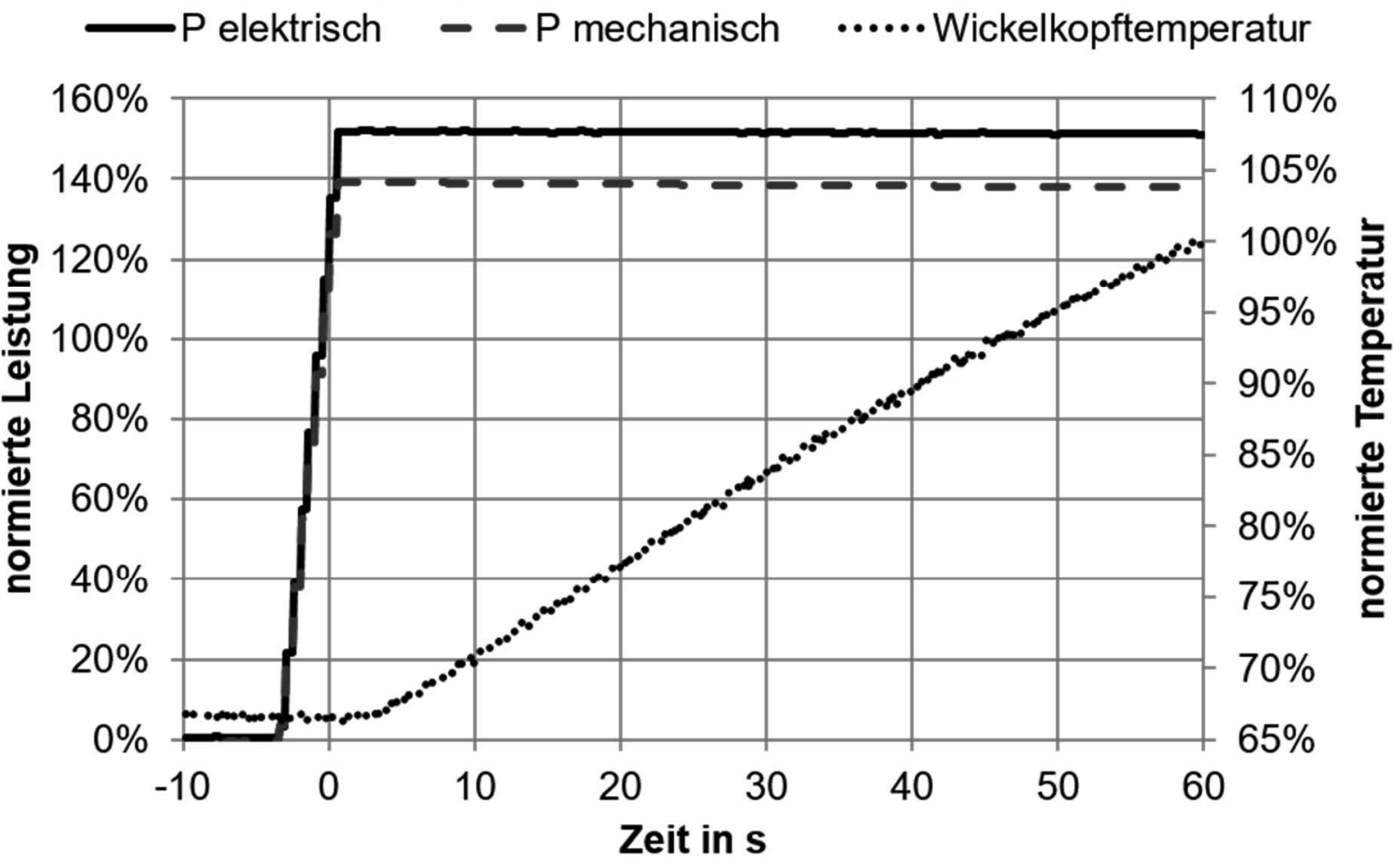

Abbildung 3-7: Beispiel der am Prüfstand ermittelten Daten

Anhand der Daten lässt sich das Ansprechverhalten der Maschine erkennen. Um die gewünschte Leistung einzustellen, sind etwa vier Sekunden erforderlich. Die Genauigkeit der Messung ist dem Anhang B zu entnehmen. Der Prüfstand erreicht bei mittleren Frequenzen die höchste Genauigkeit. So kann beispielsweise bei $400 \mathrm{~Hz}$ die Verlustleistung $P_{v}$ von $6.805 \mathrm{~W}$ mit einer Unsicherheit von $U_{0,95}=20,41 \mathrm{~W}$ bestimmt werden. Für die Ermittlung des Widerstandserhöhungsfaktors ist die Differenz zwi- 
schen gemessener elektrischer und mechanischer Leistung relevant, welche die Verlustleistung ergibt:

$$
P_{v}=P_{e l}-P_{m e c h}
$$

Die so ermittelte Gesamtverlustleistung muss gemäß den in Abschnitt 2.2.2 genannten Verlustarten aufgegliedert werden, um die Kupferverluste bestimmen zu können:

$$
P_{v}=P_{c u}+P_{F e}+P_{r e i b}+P_{m a g}
$$

Um die verschiedenen Verlustarten von vornherein zuverlässig separiert zu bestimmen, gibt es derzeit noch keine messtechnischen Vorrichtungen. Daher ist an dieser Stelle ein Behelf notwendig. Die Unbekannten in Gleichung (3-6) werden mit Hilfe anderer Informationsquellen bestimmt. Die Schwäche dieses Vorgehens ist, dass sich die Messunsicherheiten der einzelnen Größen aufsummieren.

Die in den Permanentmagneten auftretenden Verluste $P_{\text {mag }}$ werden mit Hilfe einer 3D-Finite-Elemente-Berechnung bestimmt. Ihre Zuverlässigkeit ist aus Vorversuchen bekannt, die dem Anhang $\mathrm{C}$ zu entnehmen sind. Einen Unsicherheitsbeitrag verursacht der Betrieb am Umrichter. Hierdurch entstehen zusätzliche Verluste, die einen signifikanten Betrag erreichen können, wie Islam in seiner Arbeit [21] anhand einer Asynchronmaschine mit Käfigläufer aufzeigt. Er betrachtet den Einfluss auf den Widerstandserhöhungsfaktor. Aus Petermaiers Untersuchungen [42] der Verluste in Permanentmagneten beim Umrichterbetrieb einer permanenterregten Synchronmaschine geht hervor, dass eine Zwischenkreisspannung nahe an der Strangspannung von Vorteil ist, um die zusätzlichen Verluste gering zu halten. Dies wird bei der Versuchsplanung der hier vorliegenden Untersuchung berücksichtigt und es wird ein Verhältnis von Zwischenkreisspannung zur effektiven Strangspannung von 0,8 realisiert. Auf diese Art können die zusätzlichen Verluste reduziert werden. Dennoch stellen sie bei dem vorliegenden Versuchsaufbau einen signifikanten Unsicherheitsbeitrag dar. Bei maximaler Frequenz von $933 \mathrm{~Hz}$ erreichen die Magnetverluste $P_{\text {mag }}$ einen Wert von $448 \mathrm{~W}$ bei einer Unsicherheit von $U_{0,95}=44,78 \mathrm{~W}$. Ähnlich wie bei den Magnetverlusten wird auch bei den im Blechpaket auftretenden Verlusten $P_{F e}$ vorgegangen. Diese werden mittels 2D-Finite-Elemente-Methode berechnet. Zusätzlich kommt auch hier ein Vorversuch zum Tragen. Bei diesem wird die elektrische Maschine im Leerlauf betrieben, um die auftretenden Verluste zu messen. Darüber hinaus wird die E-Maschine ohne Permanentmagnete angetrieben, um die Reibungsverluste $P_{\text {reib }}$ zu messen. Der Vorversuch findet sich in Anhang D. Aus der Differenz kann auf die Reibverluste und die Eisenverluste im Leerlauf geschlossen werden. Die Eisenverluste können mit der Finite-Elemente-Berechnung abgeglichen werden, um so Rückschlüsse auf die Eisenverluste im Lastfall zu ziehen. Relevante Unsicherheitsbeiträge entstehen zum einen durch den Umrichterbetrieb, zum anderen durch lastunabhängige Nutungsoberwellenverluste und lastabhängige Wicklungsoberwellenverluste, die nicht von den Eisenverlusten separiert werden. Diese sorgen insbesondere für einen signifikanten Unsicherheitsbeitrag. So betragen die Eisenverluste $P_{F e}$ bei maximaler Frequenz $3.289 \mathrm{~W}$ mit einer Unsicherheit von $U_{0,95}=493,37$ W. Mit den durch Finite-Elemente-Berechnung und Vorversuche ermittelten Größen 
kann auf die zu bestimmenden Wechselstromverluste $P_{c u}$ in den Leitern geschlossen werden. Mit Hilfe der Gleichung (2-17) kann nun der Widerstandserhöhungsfaktor berechnet werden. Dieser beträgt mit dem hier beschrieben Vorgehen für die maximale Frequenz 3,01 mit einer Unsicherheit von $U_{0,95}=1,0435$. Dies ist insbesondere auf den hohen Unsicherheitsbeitrag der Eisenverluste zurückzuführen. Bei einer niedrigen Frequenz von $107 \mathrm{~Hz}$ ergibt sich für den $k_{r}$-Faktor beispielsweise ein Wert von 1,30 bei einer Unsicherheit von $U_{0,95}=0,0342$. Sämtliche Werte und Unsicherheitsbeiträge können dem Anhang B entnommen werden.

In Tabelle 2 ist in Form einer Übersicht dargestellt, auf welchen Wegen die für die Ermittlung des Widerstandserhöhungsfaktors notwendigen Größen ermittelt wurden:

\begin{tabular}{c|ccc} 
& $\begin{array}{c}\text { Analytische } \\
\text { Berechnung }\end{array}$ & $\begin{array}{c}\text { Finite-Elemente- } \\
\text { Rechnung }\end{array}$ & $\begin{array}{c}\text { Prüfstands- } \\
\text { ermittlung }\end{array}$ \\
\hline $\mathbf{P}_{\text {mech }}$ & Nicht notwendig & FEM & Prüfstandsmessung \\
$\mathbf{P e l}_{\text {el }}$ & Nicht notwendig & Prüfstandsarbeitspunkt & Prüfstandsmessung \\
$\mathbf{P}_{\mathbf{c u}, \mathbf{g}}$ & Nicht notwendig & FEM + analytisch & Messung \\
$\mathbf{P}_{\mathbf{c u}}$ & Nicht notwendig & FEM & Zu bestimmen \\
$\mathbf{P}_{\text {Fe }}$ & Nicht notwendig & Nicht notwendig & FEM + Vorversuch am Prüfstand \\
$\mathbf{P}_{\text {reib }}$ & Nicht notwendig & Nicht notwendig & Vorversuch am Prüfstand \\
$\mathbf{P}_{\text {mag }}$ & Nicht notwendig & Nicht notwendig & 3D-FEM
\end{tabular}

Tabelle 2: Übersicht über die Wege zur Ermittlung der notwendigen Verlustgrößen

\subsection{Ergebnisse}

In diesem Abschnitt werden die mittleren Widerstandserhöhungsfaktoren einander gegenübergestellt, die durch analytische Berechnung, Finite-Elemente-Methode und Messung am Prüfstand ermittelt wurden.

Die Ergebnisse sind in Abbildung 3-8 dargestellt. Dabei muss bei den Prüfstandsmessungen die Messunsicherheit berücksichtigt werden. Es fällt anhand der Messunsicherheitsbalken auf, dass diese mit steigender Frequenz stark zunimmt. Die Ursache hierfür ist der Lösungsweg. Für die Ermittlung der elektrischen Verluste in den Leitern bei Wechselstrom $P_{c u}$ sind nach Gleichung (3-6) fünf Größen erforderlich. Jede dieser Größen ist mit eigenen Messunsicherheiten behaftet, die berücksichtigt werden müssen. Darüber hinaus nehmen die meisten Größen mit der Frequenz zu, wie aus Abbildung 3-9 hervorgeht. Dadurch wachsen auch die Messunsicherheiten der Größen absolut gesehen an. Die Messunsicherheiten aller fünf Größen überlagern sich bei der Bestimmung der Messunsicherheit des Widerstandserhöhungsfaktors $k_{r}$. Jedoch ist die Genauigkeit für einen annähernden Abgleich der drei Bestimmungswege immer noch ausreichend. Weiterführendes zur Messunsicherheitsanalyse findet sich im Anhang B. Dort ist die Messunsicherheitsanalyse auf Basis von Pesch [41], Kiencke u.a. [26] und Böhme [4] durchgeführt. 


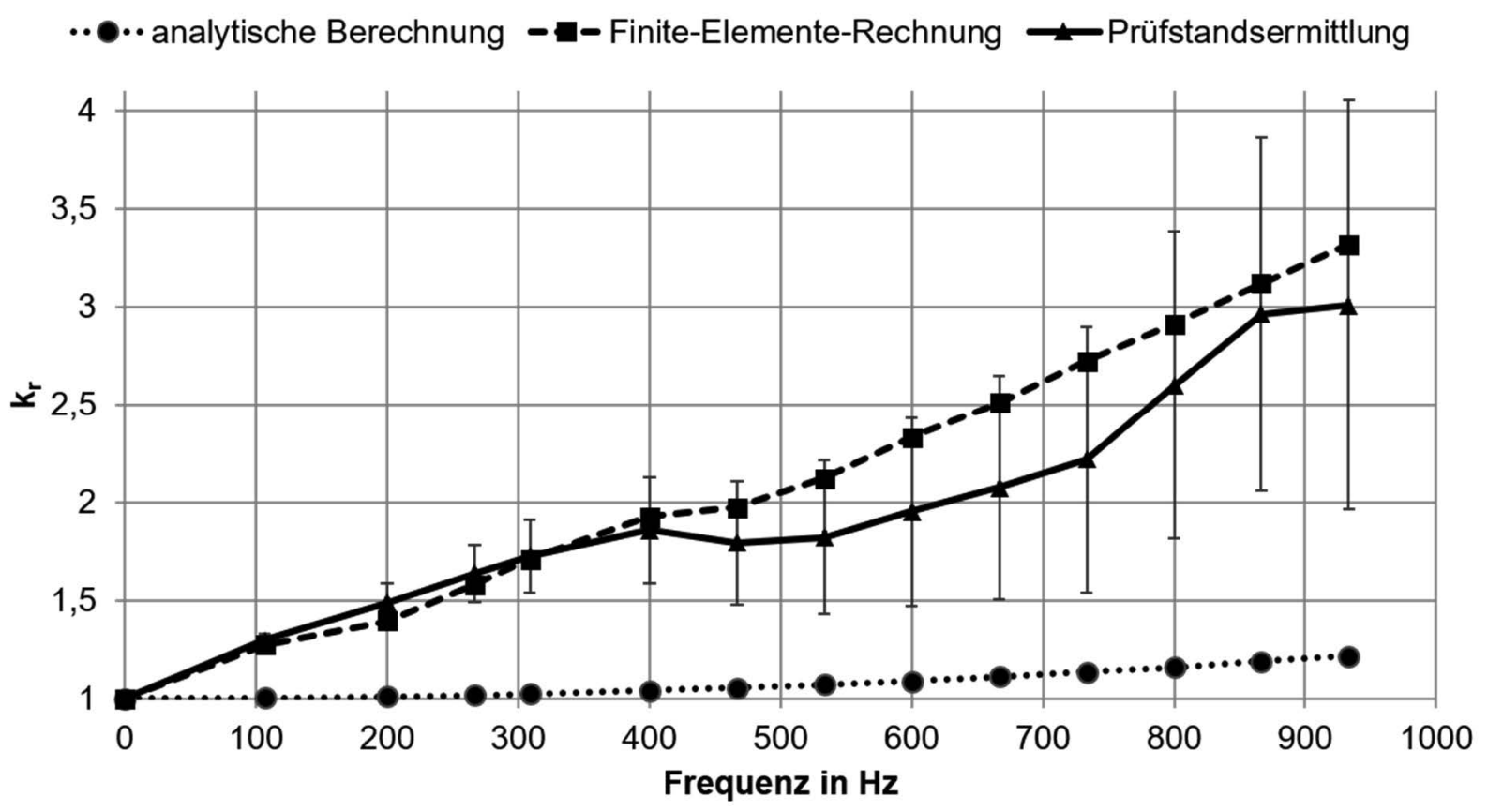

Abbildung 3-8: Gegenüberstellung des auf drei Arten ermittelten Widerstandserhöhungsfaktors der Versuchsmaschine

Abbildung 3-8 zeigt des Weiteren, dass das Resultat der analytischen Berechnung nicht wie eine Wurzelfunktion ansteigt, wie es in Abschnitt 3.2 hergeleitet wurde. Darüber hinaus ist das „Einknicken" des Widerstandserhöhungsfaktors bei Prüfstandund Finite-Elemente-Bestimmung ab $400 \mathrm{~Hz}$ ein interessanter Aspekt. Dieser Frequenzbereich liegt in der Region des Feldschwächbereichs. Hier wird eine Schwächung des Rotorfelds notwendig, um die Spannungsgrenze der Maschine weiterhin einzuhalten, wie es in Abschnitt 2.2.3.2 erläutert wird. Dadurch ändert sich die innere Phasenverschiebung, was eine Änderung des Luftspaltfelds zur Folge hat. Auf diesen Aspekt wird in Kapitel 7 detaillierter eingegangen.

Die bedeutendste Auffälligkeit der Abbildung 3-8 wurde aber noch nicht benannt: Die Abbildung zeigt, dass das analytische Ermittlungsverfahren für den Widerstandserhöhungsfaktor aus der Reihe fällt. Während Prüfstandsermittlung und FiniteElemente-Methode zu vergleichbaren Ergebnissen führen, liefert der analytische Ansatz deutlich geringere Werte. Aufgrund der Ähnlichkeit der Ergebnisse der Prüfstandsermittlung und der Finite-Elemente-Berechnung ist davon auszugehen, dass im analytischen Verfahren Ungenauigkeiten bestehen, die zumindest bei der untersuchten Maschine zu signifikanten Abweichungen führen. Diese Abweichungen besser zu verstehen und das analytische Verfahren so zu erweitern, dass es auch für hoch ausgenutzte Maschinen nutzbar wird, ist Thema der folgenden Kapitel.

In Abbildung 3-9 sind die Verluste nach Verlustarten aufgegliedert, über der Frequenz aufgetragen. In dieser Darstellung sind zwecks besserer Übersichtlichkeit keine Messunsicherheitsbalken angegeben. Ziel der Darstellung ist die qualitative Gegenüberstellung der verschiedenen Verlustarten. Die Messunsicherheiten sind aber in den Betrachtungen in Anhang B enthalten. 


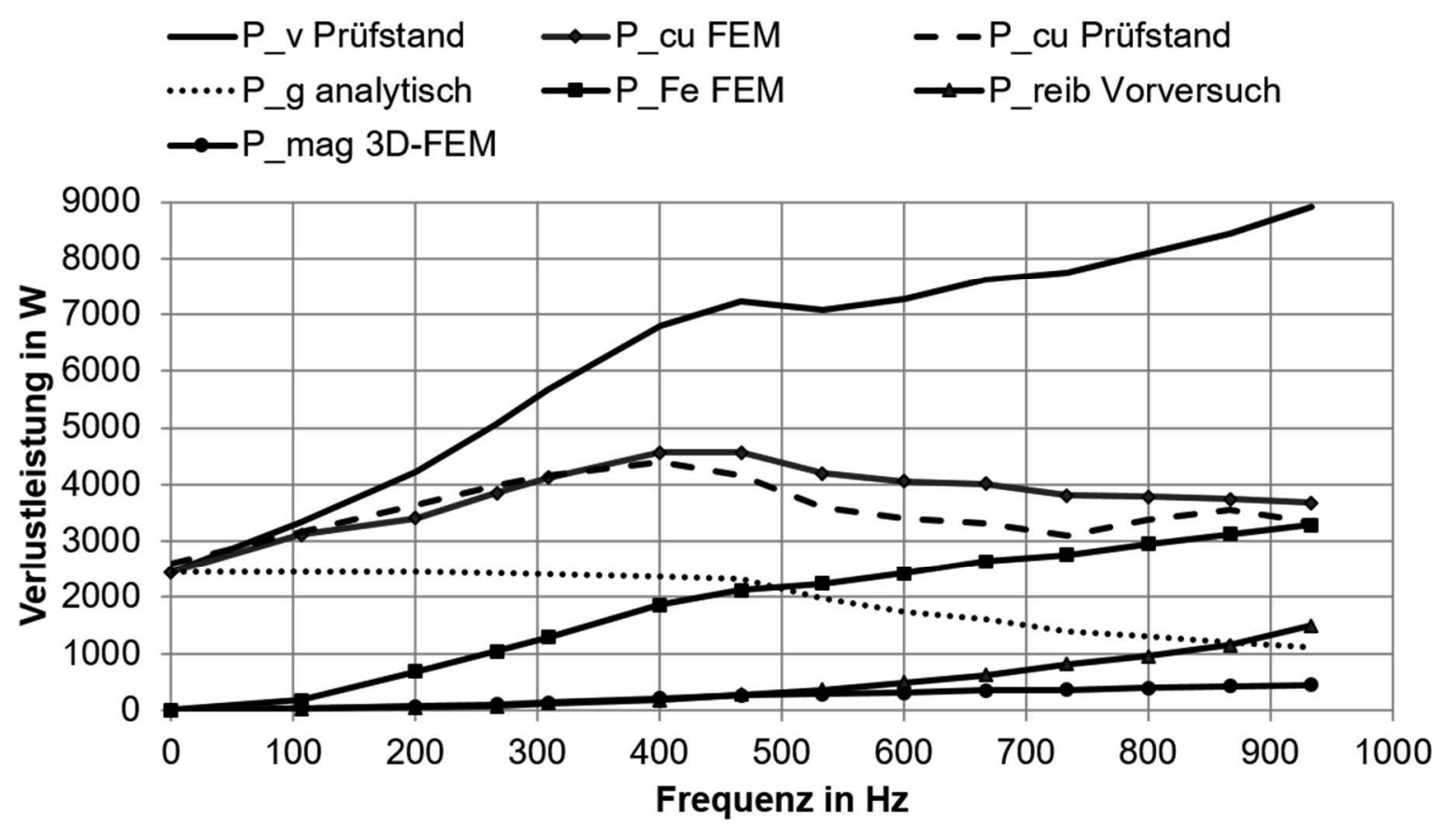

Abbildung 3-9: Verlauf und Aufgliederung der Verluste

Aus der Darstellung ist zu sehen, dass die Kupferverluste der Finite-ElementeBerechnung nicht exakt zu den Prüfstandergebnissen passen. Dies war bei dem indirekten Lösungsweg zur Auswertung der Prüfstandsergebnisse auch nicht zu erwarten. Dennoch ergibt sich ein ähnlicher Verlauf. Eine höhere Genauigkeit der Prüfstandsermittlung wäre beispielsweise bei den Eisenverlusten durch die Verwendung der bei Richter u.a. [44] beschriebenen Methode möglich gewesen. Aber auch der hier verwendete Weg reicht für die gewünschte Aussage aus. An den Kupferverlusten bei homogen verteilten Strom $P_{-} g$ analytisch ist gut der Eintritt in den Feldschwächbereich zu erkennen. Im Gegensatz zu den Kupferverlusten bei homogen verteiltem Strom nehmen die tatsächlichen Kupferverluste nur geringfügig ab.

Aus Abbildung 3-10 geht hervor, dass die Kupferverluste der Versuchsmaschine bei ausschließlicher Berücksichtigung der Kupferverluste bei homogen verteiltem Strom mit steigender Frequenz auch zunehmend unterschätzt werden. 


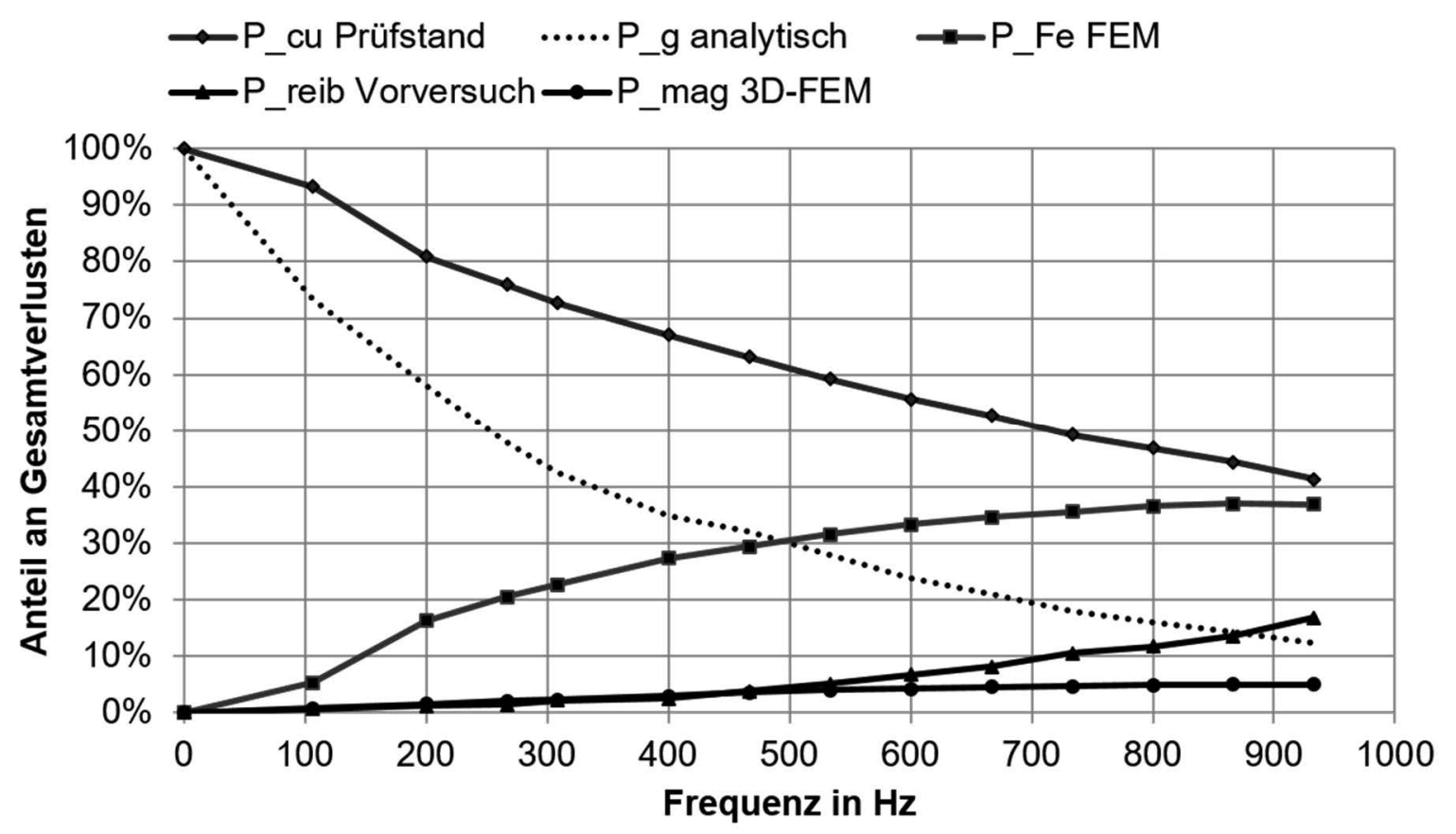

Abbildung 3-10: Anteil der Verlustarten aus dem Prüfstandsversuch an den Gesamtverlusten

Bei $933 \mathrm{~Hz}$ betragen die Kupferverluste 40\% der Gesamtverluste und nicht nur 12\%, wie die Kupferverluste bei homogen verteiltem Strom den Anschein erwecken. Die Eisenverluste steigen auf einen Anteil von knapp 40\% an. Anteilig sind die Kupferverluste stets am größten. Im vorderen Frequenzbereich machen sie zwischen $100 \%$ und $70 \%$ der Gesamtverluste aus. Bei Frequenzen bis $500 \mathrm{~Hz}$ spielen die Magnetverluste und die Reibung vernachlässigbare Rollen.

\subsection{Einfluss des Permanentmagnetfelds}

Die Diskrepanz der ermittelten Widerstandserhöhungsfaktoren legt als erste Vermutung nahe, dass das in die Nuten eindringende Permanentmagnetfeld die Ursache dafür sein könnte. Bei diesem handelt es sich um eine zusätzliche Feldkomponente, die im analytischen Modell nicht berücksichtigt wird und zusätzlich Stromverdrängung bewirkt. Die Amplitude des Permanentmagnetfelds ist stromunabhängig. So ändert sich die durch das Permanentmagnetfeld verursachte Stromverdrängung allein mit der Frequenz der Vorgänge. Dieses Verhalten deckt sich auch mit den Ergebnissen aus Abbildung 3-8, denn dort ist eine mit der Frequenz zunehmende Differenz zwischen den Ermittlungsverfahren zu beobachten. Dass diese Erklärung zu kurz greift und das Permanentmagnetfeld nicht die alleinige Hauptursache ist, lässt sich jedoch anhand von Abbildung 3-11 erklären. Die Abbildung stellt den $k_{r}$-Verlauf einer Simulation ohne Permanentmagnete dem regulären $k_{r}$-Verlauf gegenüber. Dabei ist auf der $x$-Achse die Leiterlage aufgetragen. "1" stellt den untersten und "14" den obersten Leiter in der Nut dar, entsprechend der Beschriftung in Abbildung 4-3. Auf der yAchse ist der Widerstandserhöhungsfaktor $k_{r}$ aufgetragen. Im Unterschied zur Abbildung 3-8 ist der Widerstandserhöhungsfaktor hier für jede Leiterlage einzeln angegeben und nicht gemittelt für die gesamte Spule. 


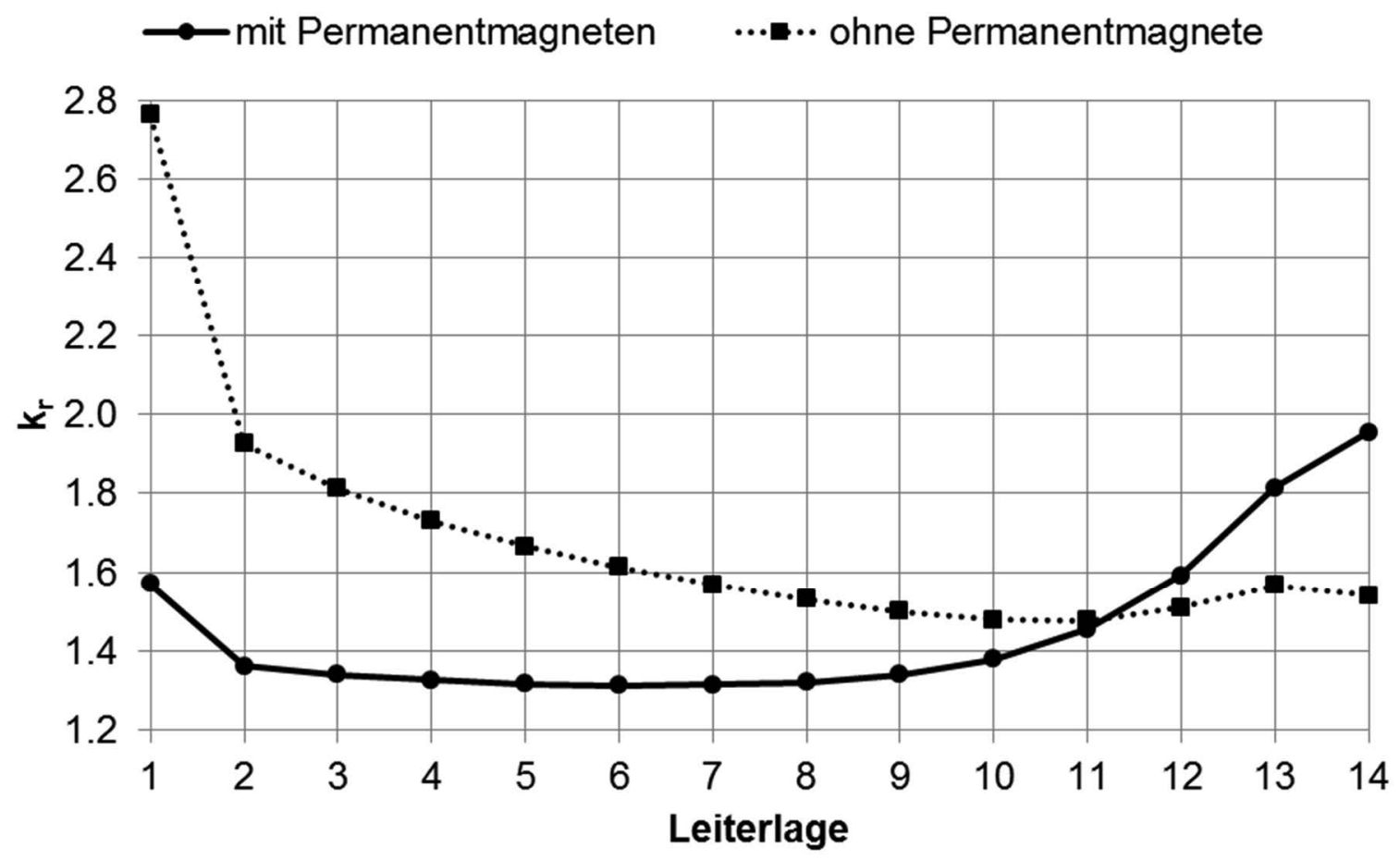

Abbildung 3-11: Gegenüberstellung von $k_{\digamma}$-Verläufen in den Leitern 1 bis 14 (siehe Abbildung 4-3) mit und ohne Permanentmagnete bei einer Frequenz von $467 \mathrm{~Hz}$ und einem Strangstrom von $419 \mathrm{~A}$

Die durchgezogene Linie in Abbildung 3-11 zeigt die reguläre Simulation des in Abschnitt 3.3 beschriebenen Maschinenmodells. Schon diese Simulation wirft in Bezug auf die zuvor genannte Vermutung Fragen auf, da sie den Anstieg des Widerstandserhöhungsfaktors im unteren Nutbereich nicht erklären kann. Denn es ist unwahrscheinlich, dass das Permanentmagnetfeld so tief in die Nuten eindringt und am Nutgrund noch an Intensität gewinnt. Weitere Fragen wirft die Simulation ohne Permanentmagnete auf. Es wäre zu erwarten, dass sich durch die fehlenden Permanentmagnete die Stromverdrängung reduziert, da die zusätzliche Feldkomponente verschwindet. Doch ohne Permanentmagnete steigt der Widerstandserhöhungsfaktor in weiten Teilen der Spule an. Dies zeigt, dass die Vermutung, die Permanentmagnete seien die Hauptursache der zusätzlichen Stromverdrängung, zu kurz greift.

Im weiteren Verlauf der Arbeit wird sich zeigen, dass die Permanentmagnete tatsächlich Einfluss auf die Stromverdrängung haben. Die Zusammenhänge dabei sind jedoch komplexer als zunächst angenommen. Des Weiteren stellen die Permanentmagnete nicht die alleinige Ursache dar. Ein Grund dafür sind auch die Zahnköpfe der untersuchten Maschine, wie sie in Abbildung 3-1 zu erkennen sind. Diese hindern das Permanentmagnetfeld am Eindringen in die Nut. 


\section{Voruntersuchung zur Abweichung des analytischen Verfahrens}

In Kapitel 3 wurde gezeigt, dass das in Abschnitt 2.1.4 beschriebene analytische Verfahren für die untersuchte Maschine keine zuverlässigen Ergebnisse liefert. Ziel der folgenden Kapitel ist es, die Ursachen dafür aufzuzeigen und das analytische Verfahren so zu erweitern, dass es auch auf hoch ausgenutzte E-Maschinen anwendbar ist.

Am Ende von Kapitel 3 konnte das stromunabhängige Permanentmagnetfeld bereits als alleinige Hauptursache der zusätzlichen Stromverdrängung ausgeschlossen werden. In Kapitel 4 wird eine Voruntersuchung beschrieben. Diese hat zum Ziel, die magnetischen Feldverhältnisse in der Nut als Ursache der Stromverdrängung genauer zu betrachten und dadurch einen tiefergehenden Abgleich zwischen analytischem und Finite-Elemente-Lösungsweg zu ermöglichen. Der Kern von Kapitel 4 ist die Überprüfung der Randbedingung eines reinen Nutquerfelds. Dies wird bei dem bisherigen analytischen Verfahren vorausgesetzt [9] [11] [34]. Dazu werden in Abschnitt 4.1 zunächst Vorüberlegungen angestellt, die die Annahme eines reinen Nutquerfelds auf Plausibilität prüfen. Darauf aufbauend werden in Abschnitt 4.2 das verwendete Finite-Elemente-Grundmodell und die abgeleitete Untersuchungsmethode vorgestellt. In Abschnitt 4.3 werden die Ergebnisse der mit dem Finite-Elemente-Grundmodell durchgeführten Untersuchungen gezeigt und diskutiert und in Abschnitt 4.4 zusammengefasst.

\subsection{Vorüberlegungen}

In diesem Abschnitt werden mögliche Ursachen für die Abweichung des analytischen Verfahrens eingegrenzt. Dazu werden zunächst Annahmen und Voraussetzungen des verwendeten analytischen Modells, das in Abschnitt 2.1.4 beschrieben wurde, näher betrachtet.

Bei Betrachtung der gegossenen Spulen der in Kapitel 3 untersuchten elektrischen Maschine fällt das ungewöhnliche Design auf, wie es in Abbildung 3-1 zu sehen ist. Die Leiter sind flach und breit ausgeführt. Sie wurden im Auslegungsprozess nach dem in den Gleichungen (2-18) bis (2-22) beschriebenen analytischen Formelwerk zur Bestimmung der Stromverdrängung optimiert. Nach diesen Gleichungen wirkt sich die Leiterhöhe negativ auf die Stromverdrängung aus, während die Leiterbreite keinen Einfluss darauf hat. Das Formelwerk beruht auf der Annahme eines reinen Nutquerfelds. Es stellt sich also die grundlegende Frage, ob die Annahme eines reinen Nutquerfelds plausibel ist. Betrachtet man zunächst Gleichung (2-14), so ist zu erkennen, dass ein geringer magnetischer Widerstand $R_{\text {mag }}$ zu einem höheren magnetischen Fluss $\phi$ führt. Das bedeutet, dass die magnetische Flussdichte größer wird, wenn der magnetische Kreis in weiten Teilen aus Elektroblech besteht. Bei den quer zur Nut verlaufenden Feldlinien nimmt der umfasste Strom vom Nutgrund zur Nutöffnung zu, während der magnetische Widerstand nur von der Nutbreite abhängt. Somit fällt nur auf den Strecken in der Nut magnetische Spannung ab. Bei parallelen Zähnen bleibt der magnetische Widerstand quer zur Nut konstant. Die magnetische Flussdichte nimmt dadurch quer zur Nut in Richtung Nutöffnung zu. 
Betrachtet man nun die magnetische Flussdichte in Längsrichtung, die in Abbildung 4-1 schematisch im Zahn und in der Nut dargestellt ist, so gilt

$$
B_{z}=\mu_{0} \mu_{r} H_{z}
$$

und

$$
B_{n}=\mu_{0} H_{z}=\frac{B_{z}}{\mu_{r}}
$$

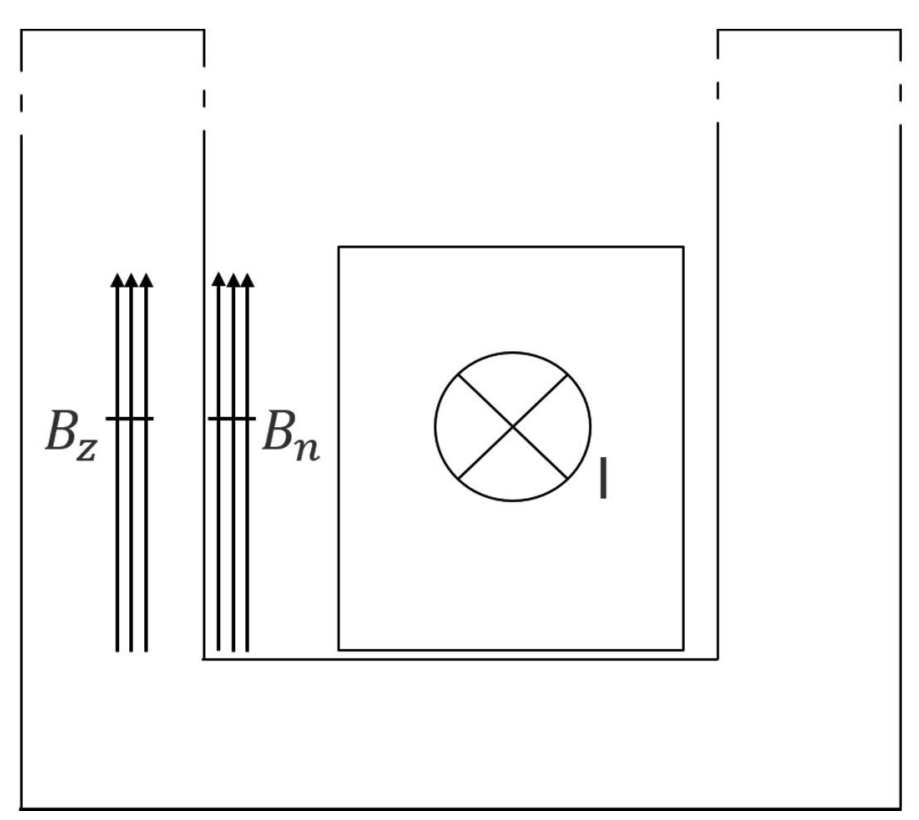

Abbildung 4-1: Vergleich der magnetischen Flussdichte in Längsrichtung im Zahn und in der Nut

Elektroblech hat im nichtgesättigten Bereich einen um den Faktor 1.000 und mehr höheren $\mu_{r}$-Wert als Luft. Das bedeutet, dass unter realen Bedingungen stets ein Längsfeld in der Nut besteht. Dieses ist jedoch um ein Vielfaches kleiner als jenes in den Zähnen. Bei dem in Abschnitt 2.1.4 beschriebenen analytischen Verfahren wird vereinfachend $\mu_{r} \rightarrow \infty$ für das Elektroblech angenommen. Dadurch entfällt das Längsfeld in der Nut. Diese Vereinfachung führt wie in Abbildung 4-2 gezeigt zu einem reinen Nutquerfeld, was zunächst zulässig erscheint. Es wird auch seit über einhundert Jahren mit dieser Annahme gearbeitet. Jedoch ist vorstellbar, dass diese Annahme für flache, breite Leiter nicht mehr uneingeschränkt gilt. 


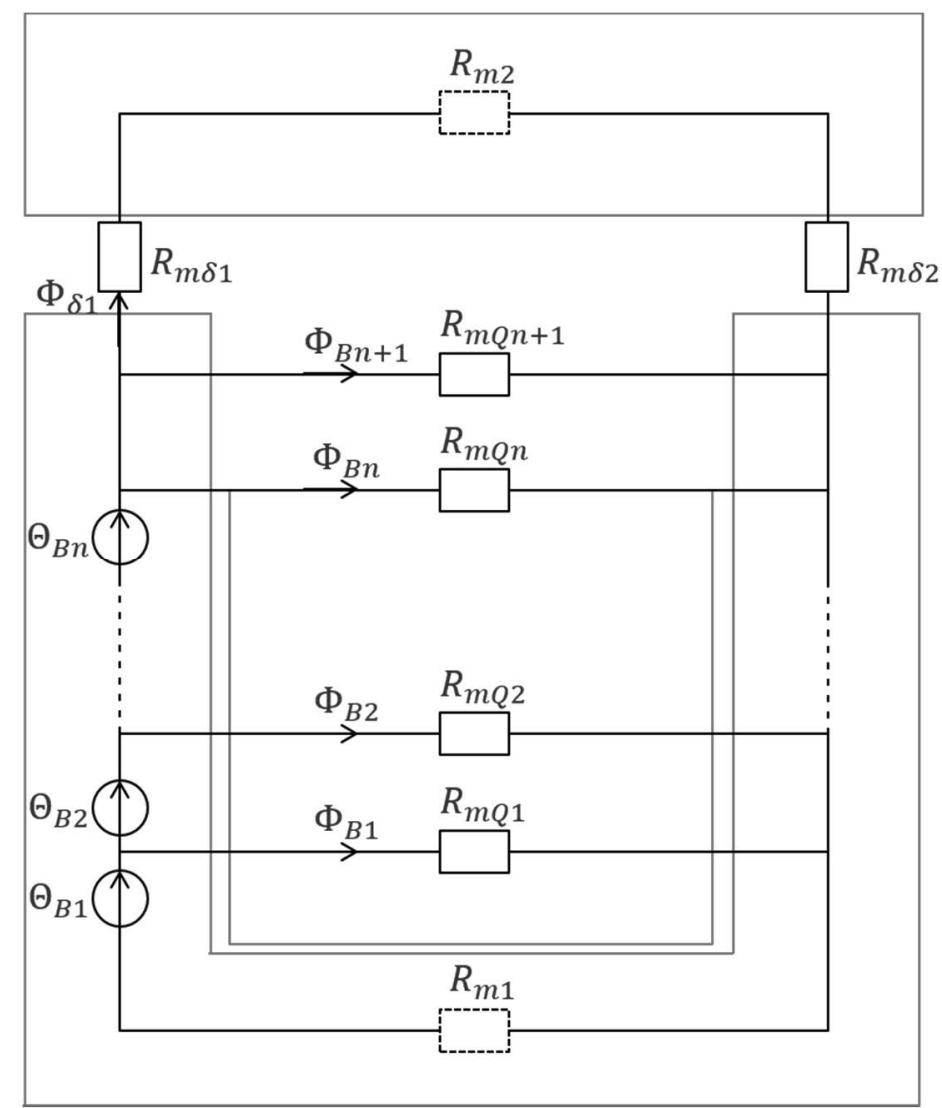

Abbildung 4-2: Vereinfachtes Ersatzschaltbild des Nutfelds als reines Nutquerfeld

Eine Untersuchung an einem einfachen Finite-Elemente-Grundmodell soll helfen, die Relation und die Größenordnung von Längs- und Querfeldanteil zu ermitteln. Ebenso kann an diesem Modell der Einfluss auf die Stromverdrängung besser abgeschätzt werden.

\subsection{Untersuchungsmethode und Finite-Elemente-Modell}

In diesem Abschnitt wird der Aufbau des Finite-Elemente-Grundmodells vorgestellt, mit dem die Relation von Längs- und Querfeldanteilen zueinander sowie deren GröBenordnungen untersucht werden. Ebenso wird mit dem Modell der Widerstandserhöhungsfaktor bestimmt, um die Ergebnisse mit dem analytischen Verfahren abgleichen zu können. Grundlegende Informationen zur Finite-Elemente-Methode finden sich in Abschnitt 2.3.

Die Geometrie des verwendeten Finite-Elemente-Modells ist in Abbildung 4-3 schematisch dargestellt. Sie bildet das vereinfachte Modell einer elektrischen Maschine nach, wie es für das in Abschnitt 2.1.4 beschriebene analytische Bestimmungsverfahren der Stromverdrängung verwendet wird. Es werden zunächst ähnliche Randbedingungen angenommen, wie sie auch für das analytische Verfahren getroffen wurden. Das für den Stator verwendete Eisen ist idealisiert angenommen. Es verhält sich bis 1,91 T wie NO30-Blech von C.D. Wälzholz und behält ab diesem Punkt ein konstantes $\mu_{r}$. Es geht jedoch nicht gegen unendlich, wie es bei dem analytischen Verfahren der Fall ist. Der Stapelfaktor beträgt $96 \%$. Die modellierten Leiter orientieren sich an den gegossenen Spulen, die in der in Kapitel 3 untersuchten Ma- 
schine verwendet werden. Sie sind flach und breit ausgeführt. Im Unterschied zur Versuchsmaschine wird nur eine Spulenseite in der Nut angenommen. Bei der Versuchsmaschine wurden Zahnspulen eingesetzt, so dass stets zwei Spulenseiten nebeneinander in der Nut liegen, die wegen des Sonderfalls der hier gewählten Lochzahl von $q=1 / 2$ immer phasenverschobene Ströme führen. Dies wird hier zunächst vernachlässigt. Ebenso wird der Rotor entsprechend dem analytischen Modell nicht betrachtet.

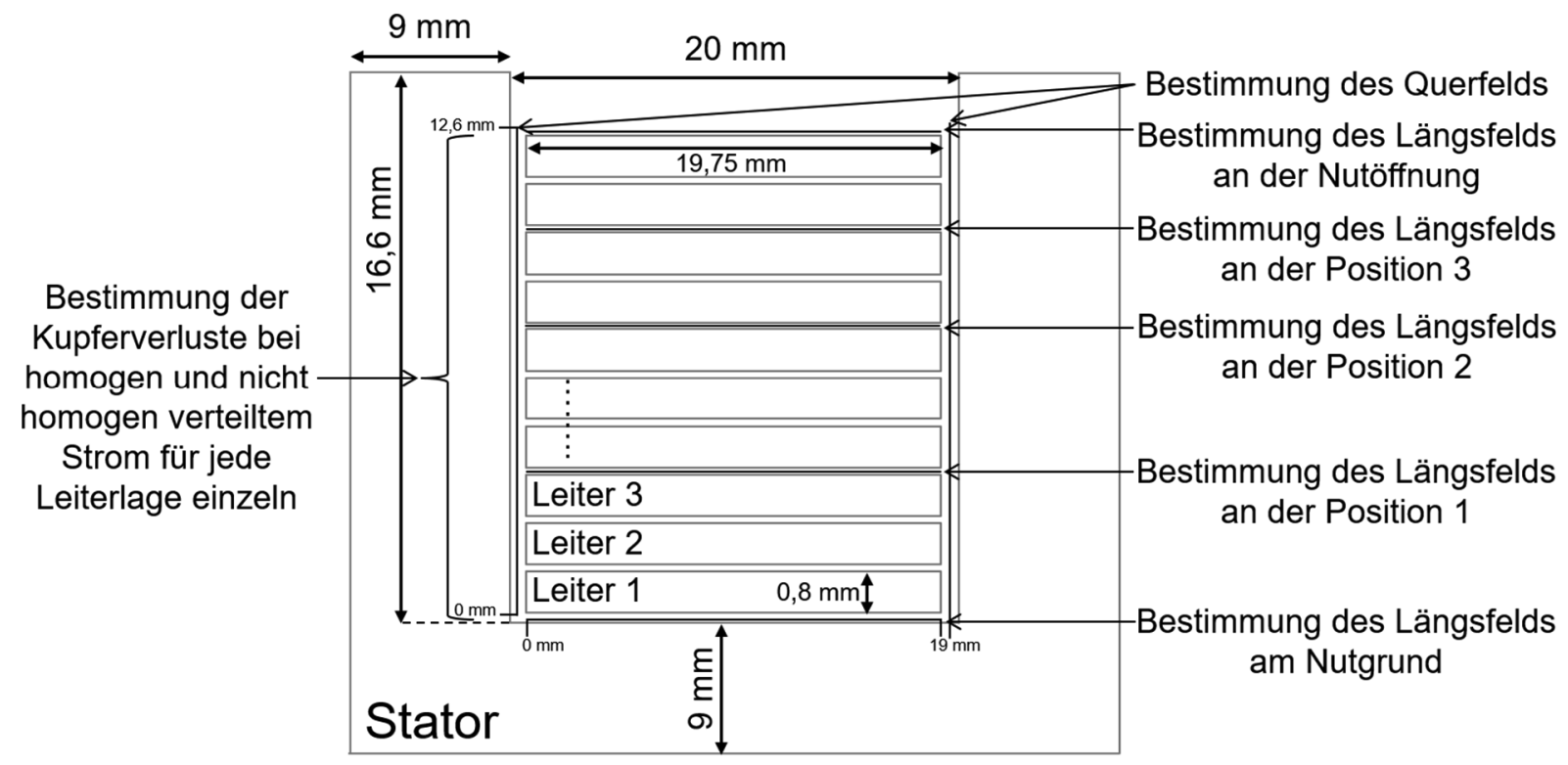

Abbildung 4-3: Übersicht über das Finite-Elemente-Grundmodell

Als Spulenmaterial wird reines Kupfer bei $105^{\circ} \mathrm{C}$ angenommen. Abschließend ist noch zu erwähnen, dass die Nut im Modell deutlich tiefer ist als in der Abbildung 4-3. Das bedeutet, dass der Abstand der obersten Leiterlage zur Nutöffnung größer ist. Dies ist erforderlich, um der Randbedingung einer unendlich tiefen Nut annähernd gerecht zu werden. Die Zähne sind hier lediglich aus Platzgründen verkürzt dargestellt. Der Annahme unendlich langer Nuten wird das Modell durch die 2D-Simulation gerecht. Das Modell wird mit 12.490 Elementen vernetzt. Analog zu Abschnitt 3.3 wird in den Leitern eine maximale Seitenlänge der Mesh-Dreiecke von 0,3 mm gewählt, um eine ausreichend genaue Berechnung trotz der geringen Leiterhöhe von 0,8 mm zu gewährleisten. Die Ermittlung der Stromverdrängung erfolgt ebenfalls gemäß der Beschreibung in Abschnitt 3.3. Ein Wickelkopf wird für dieses Modell entsprechend dem analytischen Modell nicht berücksichtigt. Weitere wichtige Modellparameter finden sich in Tabelle 3.

\begin{tabular}{c|c} 
Parameter & Werte \\
\hline Windungszahl & 10 \\
Parallelgeschaltete Teilleiter & 1 \\
Paketlänge & $95 \mathrm{~mm}$
\end{tabular}

Tabelle 3: Modelleigenschaften 
Um Informationen über die magnetischen Feldverhältnisse in der Nut zu erhalten, wurden die in Abbildung 4-3 angedeuteten Linien in das Modell mit aufgenommen. Auf diesen wird die magnetische Flussdichte ausgewertet. Abbildung 4-4 zeigt exemplarisch ein Diagramm, das sich dabei ergibt.

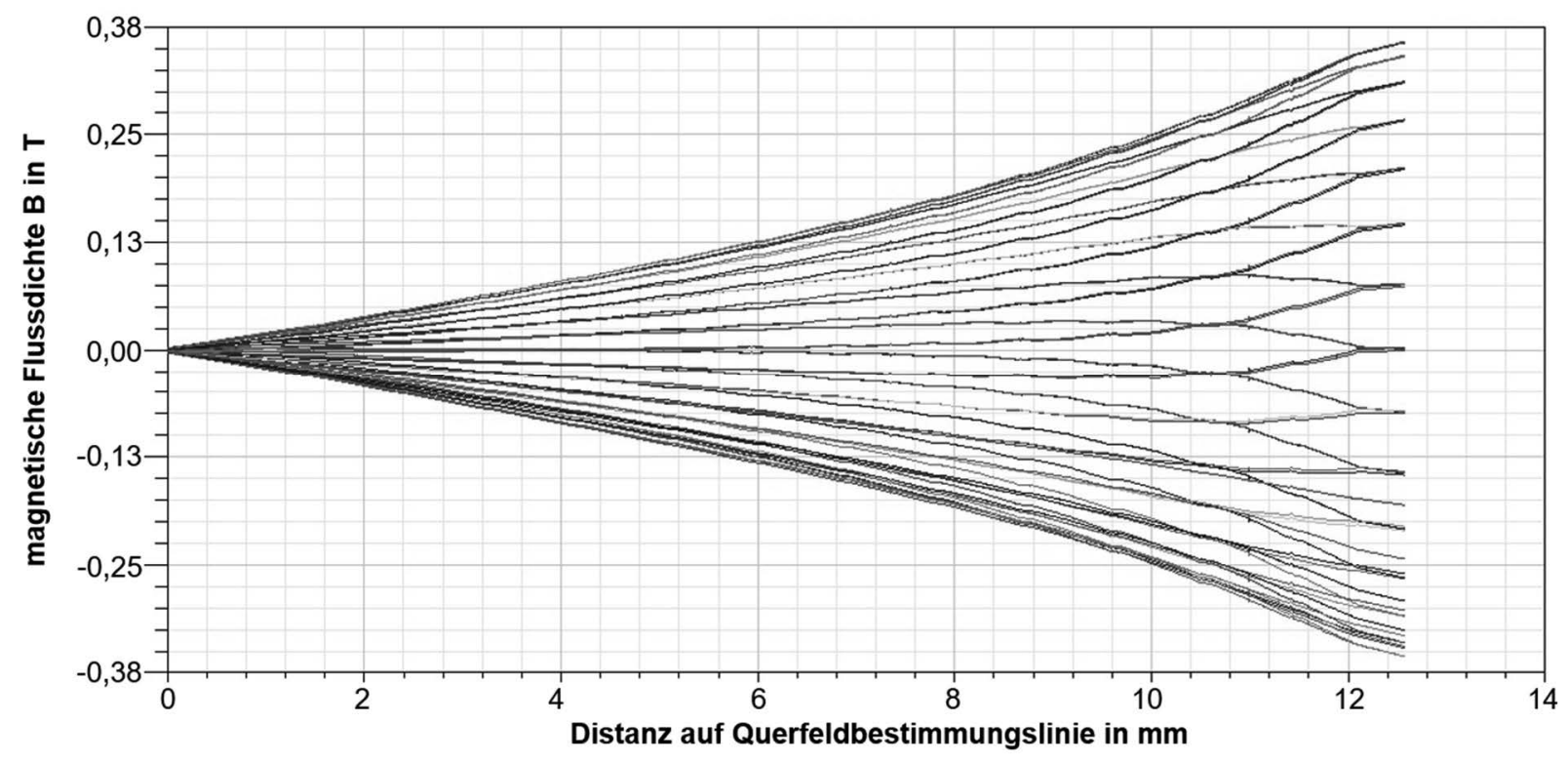

Abbildung 4-4: Exemplarische Auswertung der magnetischen Flussdichte auf der in Abbildung 4-3 gezeigten Linie zur Betrachtung des Querfelds

Es stammt von der linken vertikalen Linie zur Bestimmung des Querfelds. Auf der xAchse ist die geometrische Position in der Nut aufgetragen. Dabei stellt die Schnittstelle von $x$ - und $y$-Achse den untersten Punkt am Nutgrund dar. Der $x$-Wert gibt die Entfernung vom Linienanfang an. Der maximale $x$-Wert entspricht dem anderen Ende der Linie an der Nutöffnung. Auf der $y$-Achse ist die magnetische Flussdichte $B$ aufgetragen. Jede Linie in Abbildung 4-4 stellt einen Zeitpunkt der Simulation dar. Es wird eine Periode des eingespeisten Wechselstroms in 90 Schritten berechnet, nachdem der Vorgang eingeschwungen ist. Wie bekannt ist, nimmt das Querfeld vom Nutgrund zur Nutöffnung hin an Intensität zu. Des Weiteren ändert sich die Richtung des Felds mit dem elektrischen Strom. Dadurch ergibt sich das in Abbildung 4-4 gezeigte trichterförmige, an der $x$-Achse gespiegelte Gebilde für das Nutquerfeld. Die höchsten Werte des Nutquerfelds werden an der Nutöffnung erreicht.

\subsection{Durchgeführte Voruntersuchungen}

In Abschnitt 4.2 wird ein Finite-Elemente-Grundmodell beschrieben, das nach den Annahmen der in Abschnitt 2.1.4 beschriebenen analytischen Methode aufgebaut ist. Mit diesem werden Untersuchungen zu den magnetischen Feldverhältnissen in der Nut durchgeführt sowie ein Abgleich mit der analytischen Methode realisiert. Anders als bei dem in Abschnitt 3 durchgeführten Abgleich sind die zugrunde gelegten Modelle von Finite-Elemente-Berechnung und analytischem Verfahren in diesem Abschnitt identisch. 


\subsubsection{Betrachtung des Nutstreufelds}

Zunächst sollen Größenordnungen für die Relationen zwischen Quer- und Längsfeldanteil des Nutstreufelds ermittelt werden. Dazu wird der Strom in der FiniteElemente-Berechnung variiert und jeweils der maximale Wert des Quer- und des Längsfelds ausgewertet. Die Auswertung des Längsfelds erfolgt an den fünf in Abbildung 4-3 markierten Stellen. Die Ergebnisse sind in den Diagrammen in Abbildung 4-5 und Abbildung 4-6 dargestellt. Dabei zeigt Abbildung 4-5 die Auswertung des Querfelds. Es ist gut zu erkennen, dass die magnetische Flussdichte quer zur Nut linear mit dem Strom zunimmt. Zu jedem Strom ist der jeweilige Maximalwert der magnetischen Flussdichte aufgetragen.

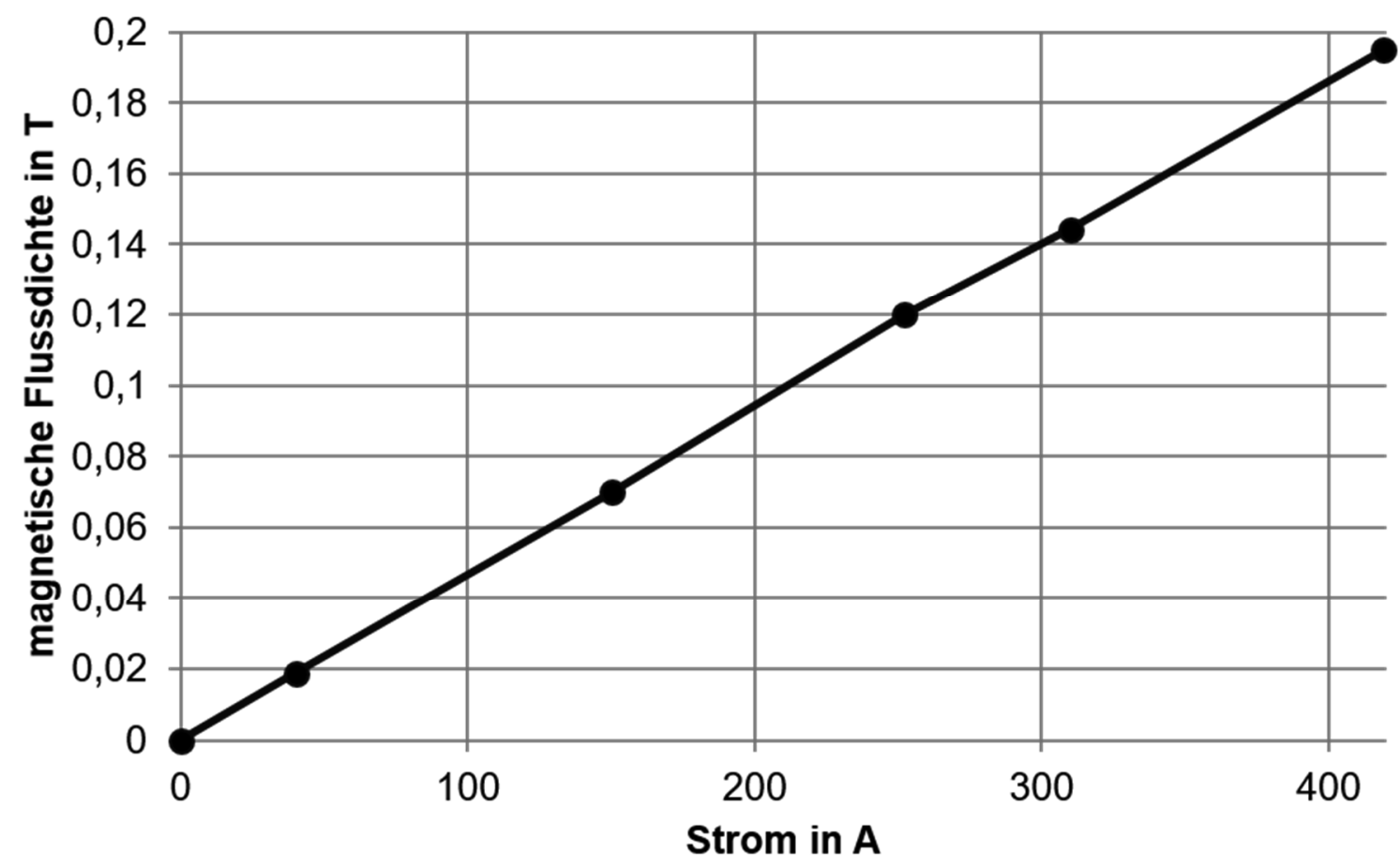

Abbildung 4-5: Veränderung des maximalen Querfeldwerts mit dem Strom

Abbildung 4-6 zeigt die magnetische Flussdichte in Längsrichtung. Die Nummerierung der Auswertelinien erfolgt vom Nutgrund zur Nutöffnung. Der "Nutgrund" ist die unterste Auswertelinie, gefolgt von "Mitte 1", "Mitte 2" und "Mitte 3". Die "Nutöffnung" stellt die oberste Auswertelinie dar. Es ist zu sehen, dass es eine Längsfeldkomponente gibt, anders als bei der für das analytische Verfahren aus Abschnitt 2.1.4 getroffenen Vereinfachung. Wie bei der magnetischen Flussdichte in Querrichtung besteht auch hier ein linearer Zusammenhang zwischen magnetischer Flussdichte und elektrischem Strom. Die Steigung der magnetischen Flussdichte mit dem Strom variiert mit der Position in der Nut. Die Ursache hierfür ist, dass das Verhältnis vom umschlossenen elektrischen Strom zum magnetischen Widerstand von der Position in der Nut abhängt. Vergleicht man die Werte der magnetischen Flussdichte aus Abbildung 4-6 mit denen aus Abbildung 4-5, so ist zu erkennen, dass die magnetischen Flussdichten in Längsrichtung nur rund $1 / 20$ der magnetischen Flussdichte in Querrichtung beträgt. Damit spielt die Längsfeldkomponente tatsächlich eine untergeordnete Rolle. Eine Vernachlässigung dieses Anteils wird in der Regel zu keiner nennenswerten Abweichung führen. Jedoch ist es eine grundlegende Erkenntnis, 
dass ein Längsfeld besteht. Dies ist auf die endliche relative Permeabilität $\mu_{r}$ zurückzuführen, die den hier durchgeführten Finite-Elemente-Berechnungen zugrunde liegt. Das analytische Verfahren basiert auf der Annahme einer unendlich hohen relativen Permeabilität, wodurch kein Längsfeld entsteht. In realen Anwendungen liegt natürlich immer eine endliche relative Permeabilität vor. Somit gibt es auch ein geringfügiges Längsfeld.

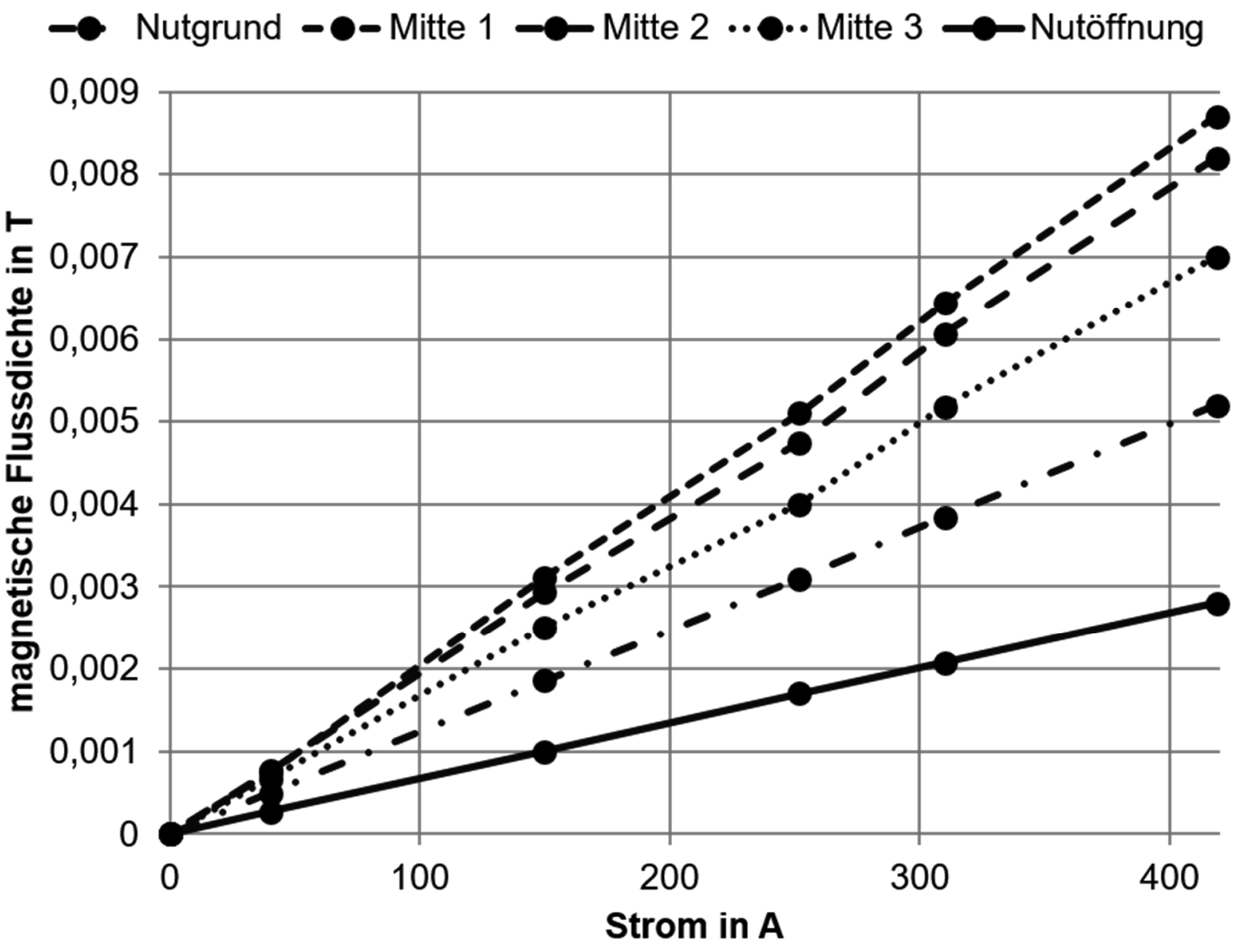

Abbildung 4-6: Veränderung der maximalen Längsfeldwerte mit dem Strom

\subsubsection{Abgleich des Widerstandserhöhungsfaktors}

Im nächsten Schritt wird nun der Widerstandserhöhungsfaktor $k_{r}$ untersucht. Verglichen wird der durch eine Finite-Elemente-Rechnung ermittelte Widerstandserhöhungsfaktor mit den Ergebnissen des analytischen Verfahrens, wie es in Abschnitt 2.1.4 geschildert wird. Mit beiden Verfahren lässt sich der Widerstandserhöhungsfaktor für jede Leiterlage separat berechnen. Abbildung 4-7 zeigt die Gegenüberstellung. Auf der x-Achse sind die Leiterlagen aufgetragen. Leiterlage 1 entspricht dem untersten Leiter in der Nut, wie es in Abbildung 4-3 angedeutet wird. Leiterlage 10 entspricht der obersten Leiterlage. Aus Abbildung 4-7 geht hervor, dass die analytische und die Finite-Elemente-Berechnung mit den in Abschnitt 4.2 getroffenen Randbedingungen auch zu vergleichbaren Ergebnissen führen. Die maximale Abweichung beträgt $0,26 \%$. 
OFEM $\times$ analytisch

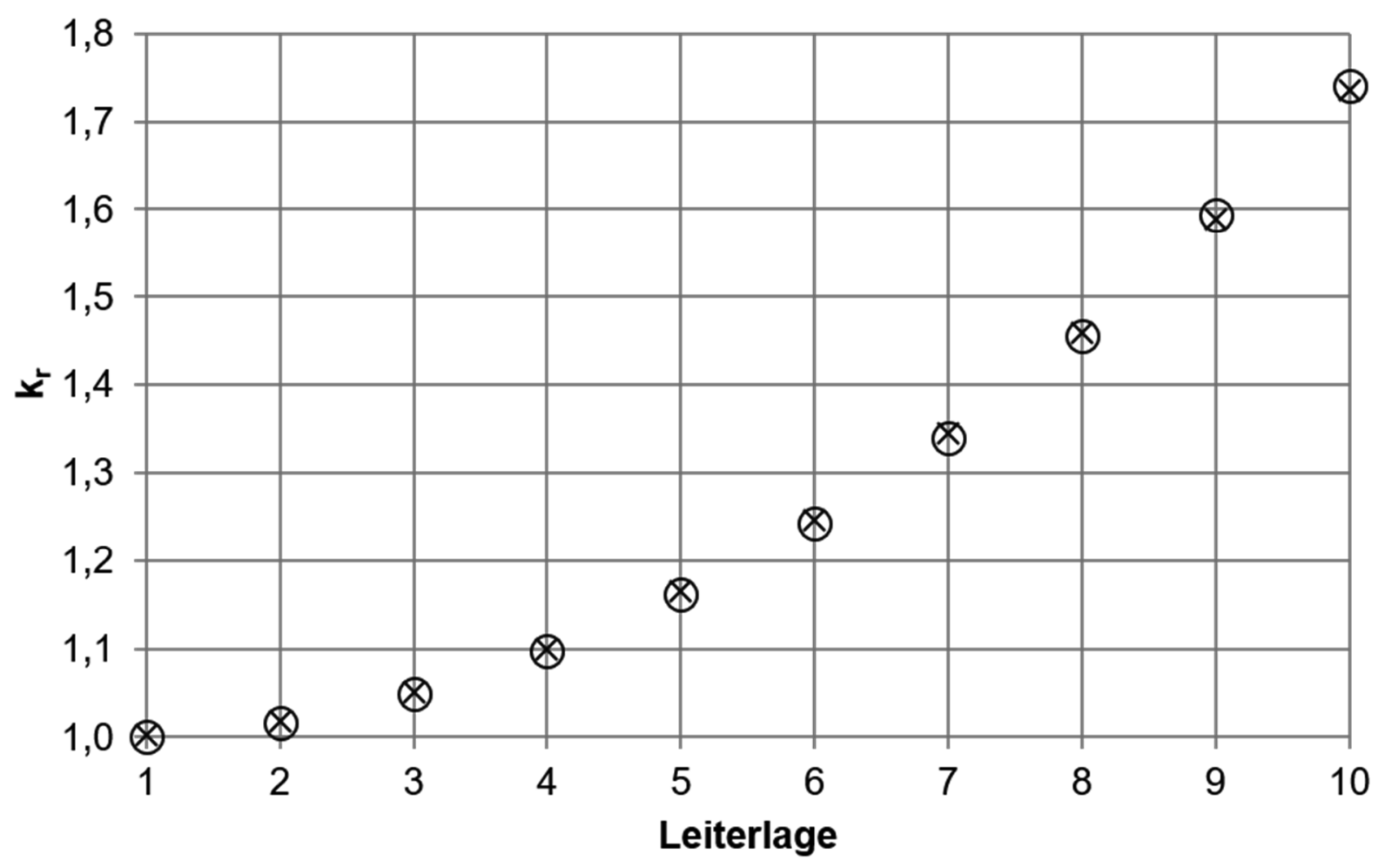

Abbildung 4-7: Abgleich des Widerstandserhöhungsfaktors bei ähnlichen Randbedingungen

In das Finite-Elemente-Modell werden im nächsten Schritt unterschiedliche Ströme in die Leiter eingespeist. Dabei wird jeweils der Widerstandserhöhungsfaktor bestimmt. Abbildung 4-8 zeigt, dass die $k_{r}$-Verläufe für alle Ströme nahezu identisch sind. Resultierend kann festgehalten werden, dass der eingespeiste Strom keinen Einfluss auf den Widerstandserhöhungsfaktor $k_{r}$ hat. Aus Abbildung 4-5 und Abbildung 4-6 ist ersichtlich, dass sich die magnetischen Flussdichten in der Nut linear mit dem Strom ändern. Dadurch wachsen die Wechselstromverluste $P_{w}$ durch eine Stromänderung in gleichem Maße an wie die Kupferverluste bei homogen verteiltem Strom $P_{g}$. In der Folge bleibt der Widerstandserhöhungsfaktor $k_{r}$ konstant und ist somit stromunabhängig. Dieses Verhalten deckt sich mit den Ergebnissen des analytischen Verfahrens. In dieses gehen zwar in Gleichung (2-18) Leiterströme ein; bei genauerer Betrachtung ist jedoch festzustellen, dass nur das Verhältnis der Leiterströme zueinander relevant ist. Denn bei einer Spule fließt in allen Leiterlagen der gleiche Strom. Dadurch kürzt sich der Strom aus der Gleichung, und es bleibt nur noch ein Verhältnis über, das etwas über die räumliche Lage der Leiter zueinander aussagt. 


\section{$419 \mathrm{~A} \Delta \quad 252 \mathrm{~A} \cdots \cdots 40 \mathrm{~A}$}

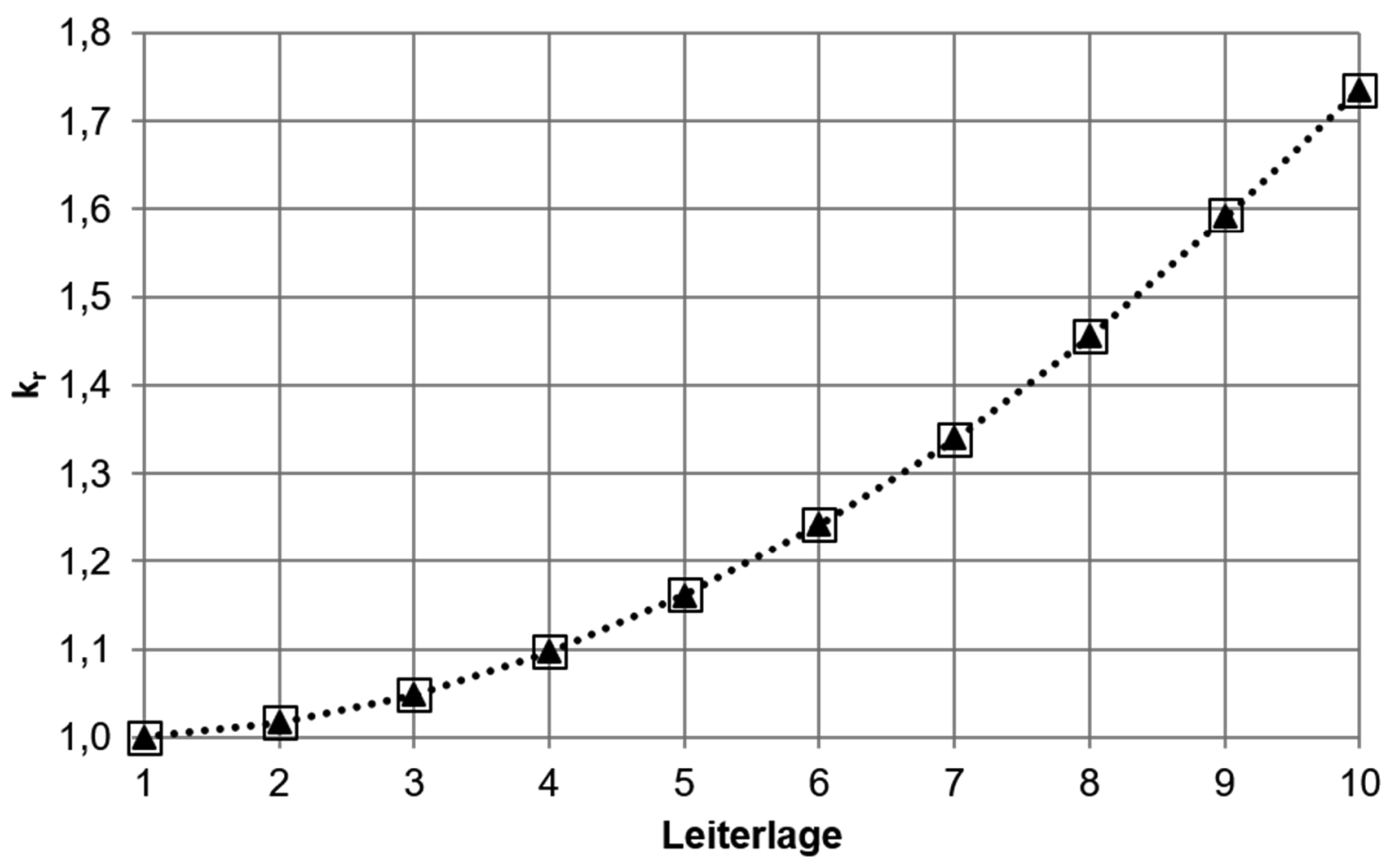

Abbildung 4-8: Widerstandserhöhungsfaktor für verschiedene eingespeiste Ströme

Aus den bisher durchgeführten Voruntersuchungen wird deutlich, dass das analytische Verfahren und die Finite-Elemente-Berechnung bei gleichen Randbedingungen zu konsistenten Ergebnissen führen. Des Weiteren spielen die Längsfeldanteile bisher tatsächlich eine vernachlässigbare Rolle. Die Diskrepanz der in Abbildung 3-8 gezeigten Abgleichergebnisse lässt sich dadurch bisher nicht erklären. Tatsächliche Maschinen, wie sie in Kapitel 3 untersucht wurden, weichen allerdings auch von den in diesem Kapitel getroffenen, idealisierten Annahmen ab. Daher ist es erforderlich, die abweichenden Randparameter tiefergehend zu untersuchen. Zunächst wird dazu die Annahme unendlich tiefer Nuten betrachtet. Diese trifft auf reale Maschinen nicht zu. Denn bei realen Maschinen werden die Nuten aufgrund des begrenzten Bauraums in hohem Maße mit Kupfer ausgefüllt. Ein großer Abstand zwischen oberstem Leiter und Nutöffnung wird daher in der Praxis nicht eingehalten. Aus diesem Grund werden im nächsten Abschnitt die Folgen von unterschiedlichen Nuttiefen untersucht.

\subsubsection{Einfluss der Nuttiefe auf das Nutstreufeld}

Um den Einfluss der Nuttiefe auf die Stromverdrängung zu untersuchen, wird diese variiert. Zunächst wird die Auswirkung auf die magnetischen Flussdichten in der Nut betrachtet. Die Ergebnisse finden sich in Abbildung 4-9. Hier ist die magnetische Flussdichte über die Position in der Nut aufgetragen. Die Positionen entsprechen den Bestimmungslinien der magnetischen Flussdichte in Längsrichtung in Abbildung 4-3. Die Bezeichnungen der einzelnen Bestimmungslinien sind in Abschnitt 4.3.1 erklärt. Die unterschiedlichen Nuttiefen sind jeweils auf einer separaten Linie in Abbildung 4-9 aufgetragen. Die Längsfeldanteile bleiben im unteren Teil der Nut relativ kon- 
stant, unabhängig von der Nuttiefe. Mit geringer werdender Nuttiefe nimmt die magnetische Flussdichte in Längsrichtung jedoch an der Nutöffnung signifikant zu. Ergänzend ist zu erwähnen, dass die durchgeführte Variation der Nuttiefe keinen Einfluss auf die Amplitude des Querfelds hat.

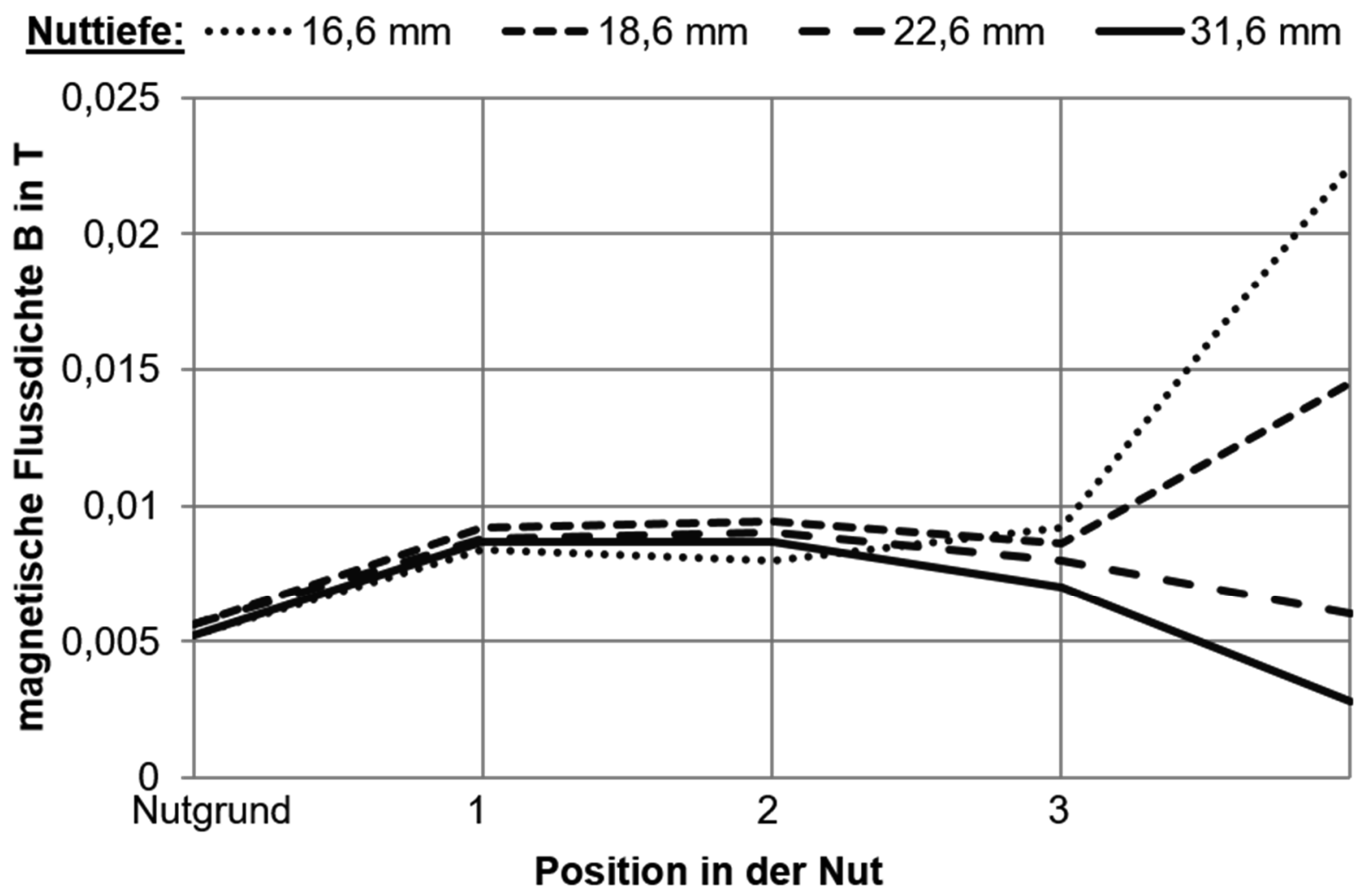

Abbildung 4-9: Magnetische Flussdichten in Längsrichtung in Abhängigkeit von der Nuttiefe

Zur besseren Beurteilung der Änderungsrate der magnetischen Flussdichte an der Nutöffnung ist diese in Abbildung 4-10 über dem Abstand des obersten Leiters zur Nutöffnung aufgetragen. Es ergibt sich ein Verlauf, der einer Potenzfunktion mit negativem Exponenten ähnelt. Ähnliche Betrachtungen erscheinen bereits bei Richter [50] und Islam u.a. [22] [20] [21], die ebenfalls den Einfluss des Abstands der obersten Leiterlage zur Nutöffnung auf die Stromverdrängung untersuchen. Dabei liegt das Hauptaugenmerk bei beiden jedoch auf der Wechselwirkung mit dem Rotor. Der Unterschied ist, dass in der vorliegenden Arbeit erstmals eine Betrachtung anhand des einfachen Grundmodells nach Abbildung 2-1 gemacht wird. Daraus ergibt sich, dass der Abstand des obersten Leiters zur Nutöffnung auch ohne Rotor Einfluss auf die magnetische Flussdichte in Längsrichtung hat, da die Feldlinien im oberen Nutbereich nicht mehr waagerecht verlaufen.

Die durchgeführte Untersuchung zeigt, dass bereits eine Änderung der Nuttiefe für einen Anstieg der magnetischen Flussdichte in Längsrichtung nahe der Nutöffnung sorgt. Jedoch sind die erreichten Werte der Längskomponente noch immer nur $1 / 10$ so groß wie die der Querkomponente. Als nächstes wird untersucht, ob sich die Änderung der magnetischen Flussdichte in Längsrichtung auch im Widerstandserhöhungsfaktor wiederfindet. 


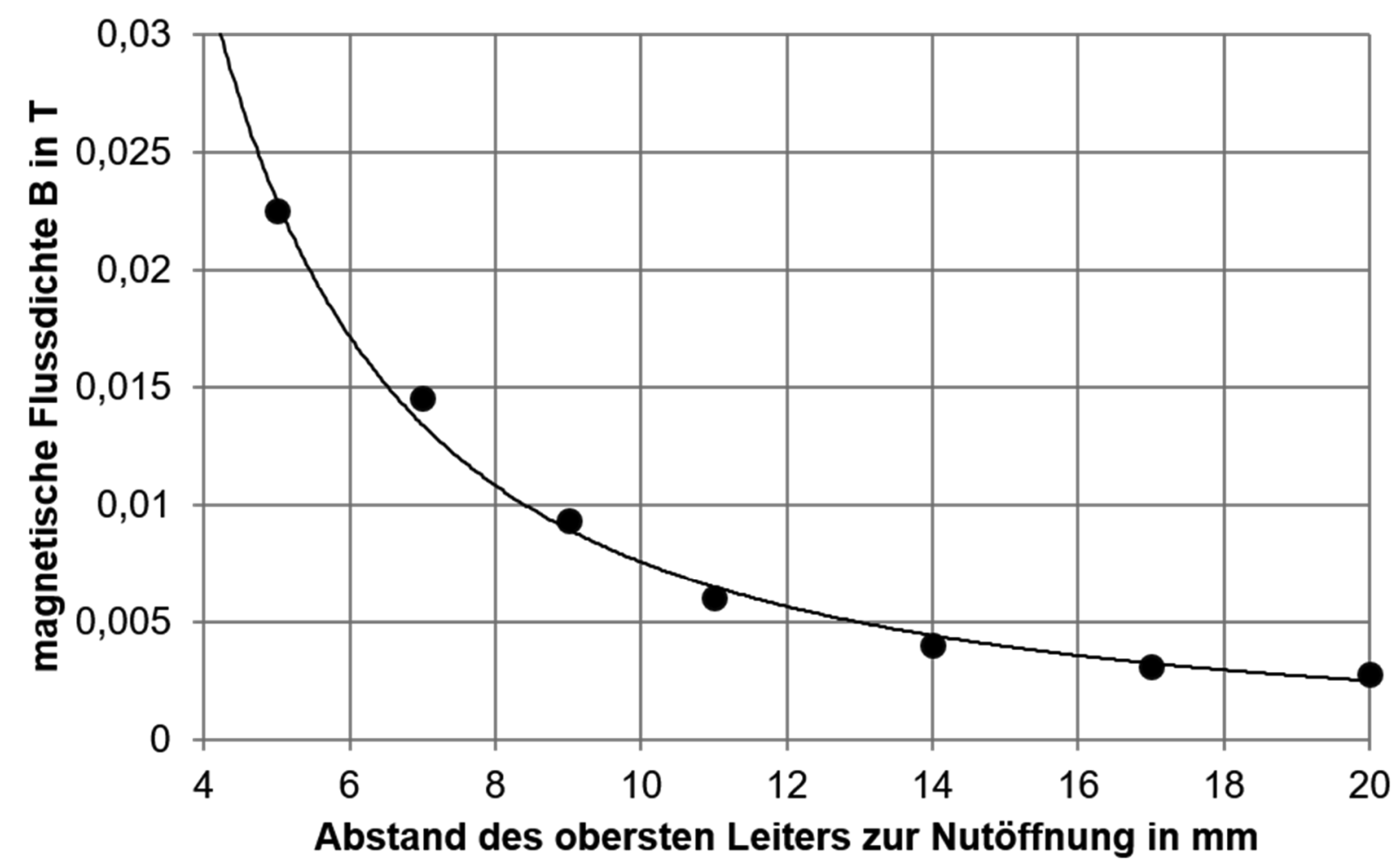

Abbildung 4-10: Magnetische Flusdichte in Längsrichtung an der Nutöffnung in Abhängigkeit von der Distanz des obersten Leiters zur Nutöffnung.

\subsubsection{Einfluss der Nuttiefe auf den Widerstanderhöhungsfaktor}

In diesem Abschnitt wird untersucht, ob sich die Längskomponente der magnetischen Flussdichte bei geringem Abstand des obersten Leiters zur Nutöffnung im Widerstandserhöhungsfaktor bemerkbar macht. Dazu wird folgendes Vorgehen gewählt: Bei einem Abstand des obersten Leiters zur Nutöffnung von $5 \mathrm{~mm}$ wird die Nut- und die Leiterbreite variiert. Dabei wird das Leiterbreiten-zu-NutbreitenVerhältnis konstant gehalten, um einen Effekt der Querfeldkomponente auf die Stromverdrängung nach Gleichung (2-22) zu vermeiden. Nach dem analytischen Modell ist dann die Leiterhöhe die einzige geometrische Größe, die Einfluss auf die Stromverdrängung hat. Die Breite des Leiters ist für die Stromverdrängung irrelevant. Da die Leiterhöhe bei dem Versuch konstant gehalten wird, dürfte sich keine Veränderung des Widerstandserhöhungsfaktors ergeben.

Die Ergebnisse der Untersuchung können Abbildung 4-11 entnommen werden. Die ersten drei Balken zeigen den Widerstandserhöhungsfaktor $k_{r}$ im obersten Leiter für drei unterschiedliche Leiterbreitenvarianten, die mittels Finite-Elemente-Rechnung bestimmt wurden. Die Zahnbreiten bleiben dabei konstant. Der letzte Balken stellt zum Vergleich die analytischen Ergebnisse des Widerstandserhöhungsfaktors im obersten Leiter dar. Da beim analytischen Verfahren die Leiterbreite keine Bedeutung für den Widerstandserhöhungsfaktor hat, gilt sie für alle Breiten. Es ist zu erkennen, dass die schmalste Variante noch dem analytischen Ergebnis entspricht. Doch mit zunehmender Leiterbreite steigt der Widerstandserhöhungsfaktor an. Der Anstieg ist gering. Er lässt sich im obersten Leiter, der am stärksten von Stromverdrängung betroffen ist, nur auf der zweiten Nachkommastelle feststellen. Die Ursache dafür ist, dass die Feldlinien im oberen Nutbereich nicht mehr geradlinig die Nut queren. Statt- 
dessen haben sie einen leicht bogenförmigen Verlauf, dessen geringer Längsfeldanteil ausreicht, die Stromverdrängung in den oberen Leiterlagen nachweislich zu beeinflussen.

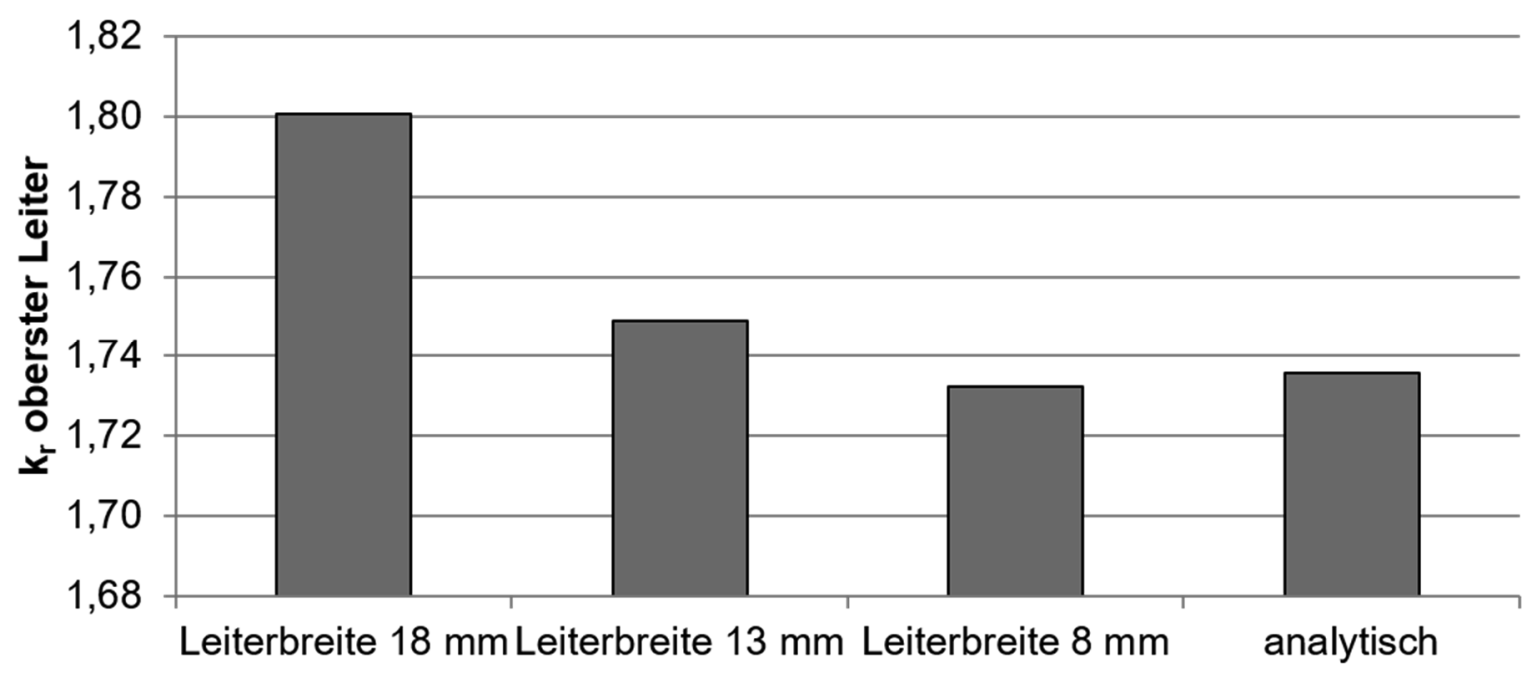

Abbildung 4-11: Widerstandserhöhungsfaktor $k_{r}$ im obersten Leiter für eine Variation der Leiterbreite bei konstantem Leiterbreiten-zu-NutbreitenVerhältnis und konstanter Zahnbreite

Der Versuch in Abschnitt 4.3.3 zeigt, dass eine realitätsnahe Nuttiefe die magnetische Flussdichte in Längsrichtung verstärkt. Aus diesem Abschnitt ergibt sich, dass bei ausreichend breiten Leitern der Widerstandserhöhungsfaktor $k_{r}$ ansteigt. Ähnlich wie hohe Leiter anfällig für Querfelder sind, sind breite Leiter offensichtlich anfällig für Längsfelder. Die Untersuchung zeigt, dass bereits eine Abweichung von den idealisierten Randbedingungen ausreichen kann, um den Widerstandserhöhungsfaktor zu beeinflussen.

\subsubsection{Einfluss der Nuthöhe und -breite auf das Nutfeld}

In diesem Abschnitt wird der Zusammenhang von Nutgeometrie und Nutfeld betrachtet. In Abschnitt 4.3.3 wurde der Einfluss der Nuthöhe auf das Nutfeld untersucht. Neben der Nuthöhe ist die Nutbreite die zweite Größe, die die Nutgeometrie definiert. Für die folgenden Betrachtungen wird das Verhältnis von Nuthöhe und -breite gebildet, um beide Größen zu erfassen:

$$
k_{\text {geom }}=\frac{h_{n}}{b_{n}}
$$

Des Weiteren wird das in Abschnitt 4.2 beschriebene Modell für die folgenden Betrachtungen variiert. Die Nuthöhe wird zwischen $9 \mathrm{~mm}$ und $34 \mathrm{~mm}$ und die Nutbreite zwischen $4 \mathrm{~mm}$ und $40 \mathrm{~mm}$ angepasst. Zusätzlich wird entsprechend Abbildung 5-4 ein magnetischer Rückschluss eingesetzt. Die Leiter werden für alle Nuthöhe-zuNutbreite-Varianten mit einem Wechselstrom von $400 \mathrm{~A}$ betrieben. Entsprechend Abbildung 4-3 wird die Längskomponente der magnetischen Flussdichte ausgewertet. Die Ergebnisse der Nutmitte sind Abbildung 4-12 zu entnehmen. 


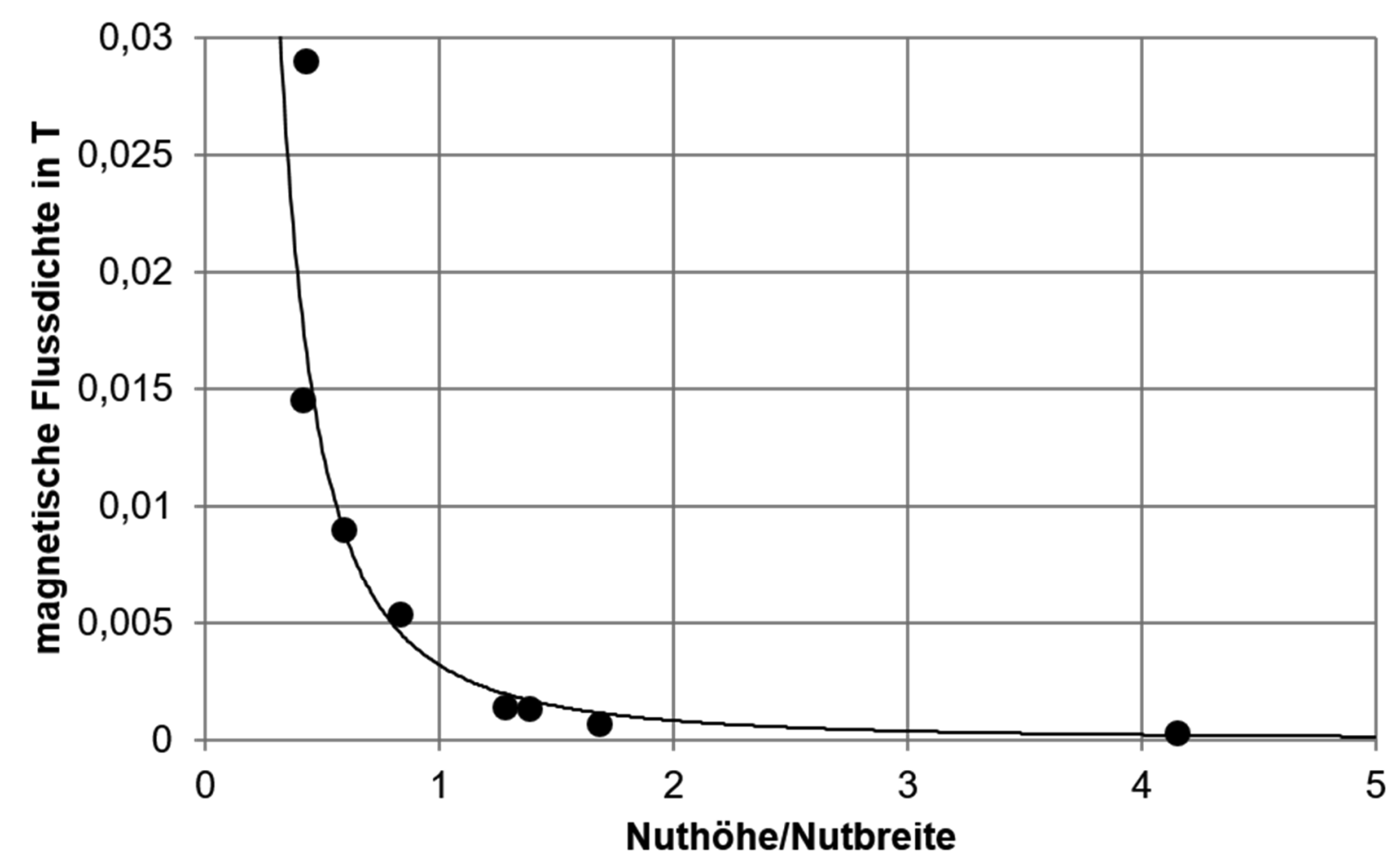

Abbildung 4-12: Längskomponente der magnetischen Flussdichte in der Nutmitte für verschiedene Nuthöhe-zu-Nutbreite-Verhältnisse

Anhand der Abbildung lässt sich die Annahme eines reinen Nutquerfelds beurteilen. Es ist zu erkennen, dass der Verlauf der magnetischen Flussdichte eine hohe Korrelation zu einer Potenzfunktion mit negativem Exponenten aufweist. Stellt man sich nun das idealisierte Modell der analytischen Methode aus Abschnitt 2.1.4 in dem Diagramm vor, so liegt es unendlich weit rechts auf der $\mathrm{x}$-Achse, denn es besitzt eine unendliche Nuthöhe bei endlicher Nutbreite, was zu einem unendlich hohen $\mathrm{kgeom}^{-}$ Faktor führt. Dadurch ist die Annahme eines reinen Nutquerfelds legitim. Betrachtet man die Versuchsmaschine aus Abschnitt 3.1, besitzt sie bei der mittleren Nutbreite einen $k_{\text {geom }}$-Faktor von rund 0,81. Damit liegt sie in einem Bereich, in dem die Vernachlässigung der Längsfeldkomponente fragwürdig erscheint. Die Daten der gesamten Nut sind dem Anhang E zu entnehmen. Dabei zeigt sich, dass die Längskomponente der magnetischen Flussdichte im gesamten unteren und mittleren Nutbereich einen ähnlichen Verlauf zu Potenzfunktionen mit negativem Exponenten aufweist. Für den oberen Nutbereich gibt es keinen klaren Zusammenhang zum $\mathrm{k}_{\text {geom}}$-Faktor. Hier ist eine Beeinflussung der Längskomponente durch abgelenkte Querkomponentenanteile anzunehmen.

\subsection{Erkenntnisse aus Voruntersuchungen}

Aus den Voruntersuchungen am einfachen Grundmodell lassen sich Erkenntnisse ziehen, die helfen, die grundlegenden Zusammenhänge der Stromverdrängung zu verstehen.

Zunächst zeigt sich, dass der analytische Berechnungsweg und die Ermittlung mittels Finite-Elemente-Methode bei gleichen Randbedingungen zu konsistenten Ergebnissen führen. Weiterhin konnte die Annahme eines reinen Nutquerfelds als zu- 
lässige Vereinfachung bestätigt werden, da die Längsfeldanteile bei der Voruntersuchung um den Faktor 20 geringer sind als die Querfeldkomponente. Darüber hinaus wurde aber festgestellt, dass sich Abweichungen von den idealisierten Randbedingungen des in Abschnitt 2.1.4 beschriebenen analytischen Modells auf das Nutfeld auswirken. So folgt aus einer realitätsnahen Nuthöhe, die von der idealisierten Annahme unendlich tiefer Nuten abweicht, ein Anstieg des Nutlängsfelds im oberen Nutbereich um den Faktor 9. Die Beeinflussung des Widerstandserhöhungsfaktors durch die Nuttiefe ist jedoch gering. Es konnte gezeigt werden, dass das Verhältnis von Nuthöhe zu Nutbreite die Stärke der Längskomponente im mittleren und im unteren Nutbereich beeinflusst. Je kleiner das Verhältnis wird, desto stärker wächst die Längskomponente der magnetischen Flussdichte an.

Aus den Ergebnissen der Voruntersuchung werden die folgenden beiden Thesen abgeleitet:

1. Abweichungen von den idealisierten Randbedingungen des analytischen Verfahrens haben eine Stärkung der Längsfeldkomponente zur Folge.

2. Für hoch ausgenutzte elektrische Maschinen ist die vereinfachende Annahme eines reinen Nutquerfelds nicht mehr allgemein zulässig.

Die Thesen werden in den folgenden Kapiteln überprüft. 


\section{Magnetische Sättigung}

Die Voruntersuchungen im Abschnitt 4.3.2 zur Abweichung des in Abschnitt 2.1.4 beschriebenen analytischen Verfahrens haben gezeigt, dass der Widerstandserhöhungsfaktor $k_{r}$ konstant bleibt, wenn die magnetische Flussdichte in Querrichtung linear mit dem Strom ansteigt. Die magnetische Sättigung des Eisenkreises führt zu einem nichtlinearen Verhalten der magnetischen Flussdichte, wie aus Abschnitt 2.1.3 hervorgeht. Daher ist davon auszugehen, dass die magnetische Sättigung Einfluss auf die Stromverdrängung hat. Das Thema wird in der Literatur teilweise behandelt [38] [6]. Darauf aufbauend wird in dieser Arbeit der Einfluss der magnetischen Sättigung des Eisenkreises auf die Stromverdrängung weiterführend betrachtet. Dazu werden in Abschnitt 5.1 zunächst die grundlegenden Zusammenhänge und insbesondere die Auswirkungen auf das Nutfeld geschildert. Anschließend werden in Abschnitt 5.2 Finite-Elemente-Berechnungen vorgestellt, die die Folgen der magnetischen Sättigung für das Nutfeld und die Stromverdrängung betrachten. Abschnitt 5.3 thematisiert die Berücksichtigung der magnetischen Sättigung in dem in Abschnitt 2.1.4 beschriebenen analytischen Verfahren. Abschließend gibt Abschnitt 5.4 eine kurze Zusammenfassung der gewonnenen Erkenntnisse.

\subsection{Grundlegende Zusammenhänge}

Wie in Abschnitt 2.1.3 beschrieben wird, ist der Zusammenhang zwischen magnetischer Flussdichte $B$ beziehungsweise magnetischer Polarisation $J_{\operatorname{mag}}$ und magnetischer Feldstärke $H$ beziehungsweise dem elektrischen Strom $I$ nur im nicht gesättigten Zustand eines ferromagnetischen Materials annährend linear.

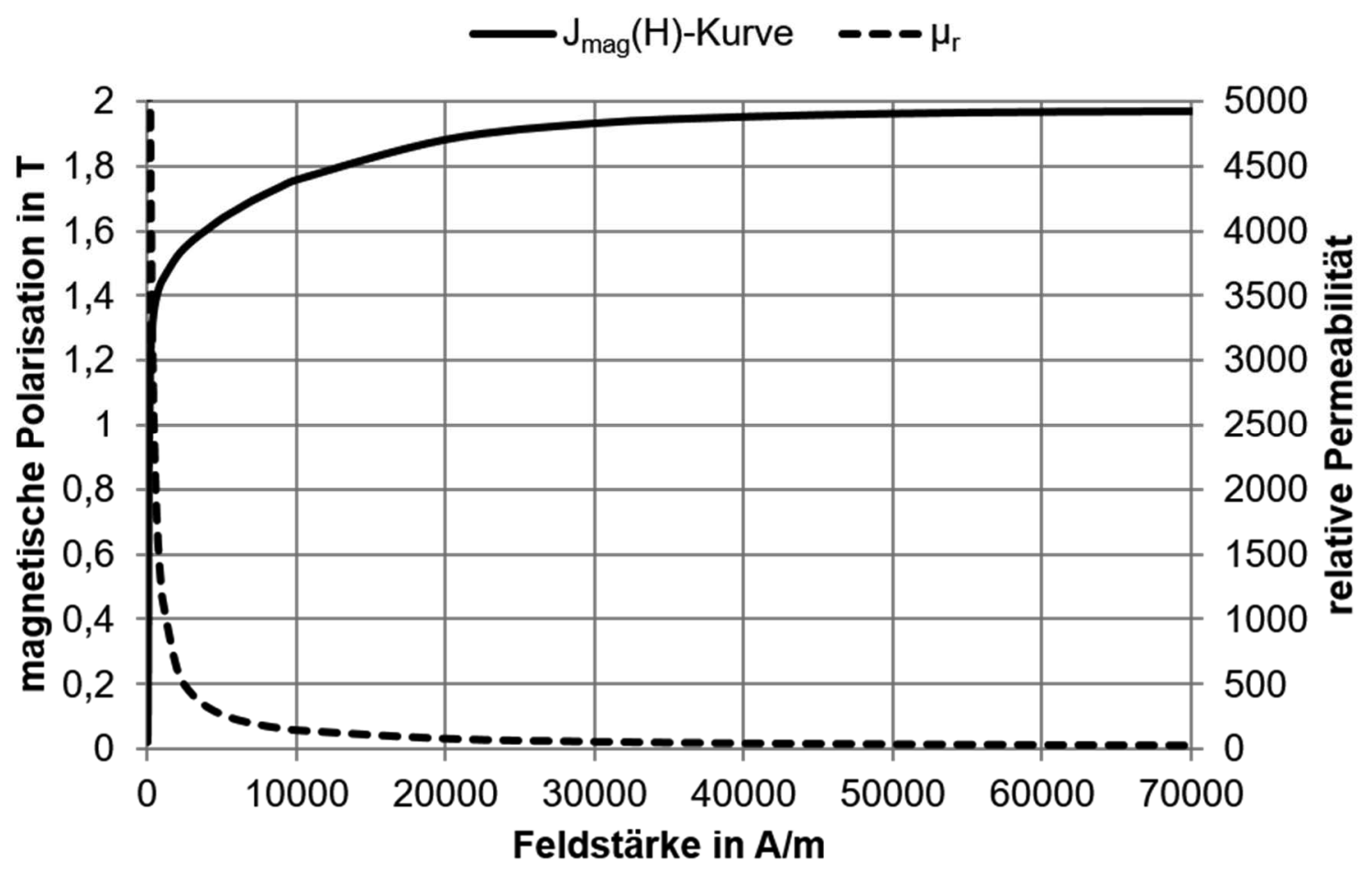

Abbildung 5-1: Zusammenhang der magnetischen Feldstärke und der magnetischen Polarisation für das verwendete NO30-Elektroblech von C.D. Wälzholz 
Abbildung 5-1 zeigt den Verlauf der magnetischen Polarisation in Abhängigkeit von der magnetischen Feldstärke in handelsüblichem NO30-Blech von C.D. Wälzholz. In der vorliegenden Arbeit werden zwei Bereiche der $J_{\text {mag }}(H)$-Kurve unterschieden: Der vordere Bereich, in dem im ferromagnetischen Material in guter Näherung ein linearer Zusammenhang zwischen magnetischer Feldstärke und magnetischer Flussdichte angenommen werden kann, reicht bis etwa 1,3 T. Der Bereich, ab dem das ferromagnetische Material keinen nennenswerten Einfluss mehr auf die magnetische Flussdichte hat, beginnt mit etwa 1,97 T. Ab diesem Punkt ist die relative Permeabilität $\mu_{\mathrm{r}}$ des Elektroblechs nur noch 15 -mal so hoch wie die von Luft. Die Magnetisierung in Gleichung (2-11) leistet dann einen geringen Beitrag zur magnetischen Flussdichte. Somit beeinflusst das Material den Zusammenhang zwischen magnetischer Feldstärke und magnetischer Flussdichte nur noch geringfügig. Dadurch gilt näherungsweise wieder der lineare Zusammenhang wie im Vakuum nach Gleichung (2-10). Der Übergangsbereich zwischen den beiden genannten Bereichen lässt sich nicht linear beschreiben. Er wird im Folgenden zusammen mit dem zweiten Bereich als Sättigungsbereich bezeichnet. Auf der rechten y-Achse der Abbildung 5-1 ist die relative Permeabilität $\mu_{r}$ aufgetragen. Diese entspricht der magnetischen Flussdichte $B$ geteilt durch die magnetische Feldstärke $\mathrm{H}$ multipliziert mit der magnetischen Feldkonstante $\mu_{0}$. Die Kurven gelten für eine Frequenz von 50 $\mathrm{Hz}$. Die Frequenz beeinflusst zwar den Verlauf der Kurven; der Einfluss ist für die hier betrachteten Zusammenhänge bei den untersuchten Frequenzen aber vernachlässigbar.

Der nichtlineare Zusammenhang zwischen magnetischer Feldstärke und magnetischer Flussdichte wirkt sich auf zwei Arten auch auf die Stromverdrängung in der elektrischen Maschine aus, die in den beiden folgenden Unterabschnitten beschrieben werden.

\subsubsection{Verminderung der Querfeldkomponente}

Die magnetische Sättigung des Eisenkreises erhöht dessen magnetischen Widerstand. Die in Abbildung 4-2 angedeuteten Widerstände $R_{m 1}$ und $R_{m 2}$ können dadurch nicht mehr vernachlässigt werden, da $\mu_{r}$ im Sättigungsbereich stark abnimmt. Infolgedessen nimmt nach Gleichung (2-14) der magnetische Widerstand des Eisenkreises zu, wodurch sich der magnetische Fluss nach Gleichung (2-15) reduziert. Dies betrifft auch die Streufeldanteile wie das Nutquerfeld. Wie in Abschnitt 4.3 gezeigt wurde, bleibt der Widerstandserhöhungsfaktor $k_{r}$ nur konstant, wenn die magnetische Flussdichte linear mit dem elektrischen Strom zunimmt. Da die magnetische Flussdichte in Nutquerrichtung durch den beschriebenen Effekt sinkt, verringert sich zunächst auch die Stromverdrängung.

Der hier genannte Zusammenhang wird bereits bei Dabrowski u.a. [6] angedeutet. Er schlägt vor, die Sättigung des Eisens über das Leiterbreite-zu-Nutbreiteverhältnis in Gleichung (2-22) zu berücksichtigen.

\subsubsection{Zusätzliche Längsfeldkomponente durch Entlastung}

Neben der Verminderung der Querfeldkomponente führt die magnetische Sättigung zu einer Senkung der relativen Permeabilität $\mu_{r}$ des Eisenkreises. Das hat gemäß Gleichung (4-2) einen Anstieg der magnetischen Flussdichte in Längsrichtung in der Nut zur Folge. Dabei handelt es sich um die sogenannte Zahnentlastung, bei der sich 
parallel zur sättigenden Eisenstrecke ein magnetisches Feld ausbildet, das die Eisenstrecke entlastet. Das Phänomen wird beispielsweise bei Müller u.a. [34] beschrieben. Der Einfluss auf die Stromverdrängung klingt bei Oberretl [37] [38] an, wird von ihm aber nicht tiefergehend untersucht.

Die Entlastung ferromagnetischer Abschnitte durch Luftstrecken gilt nicht nur für Zähne, sondern tritt auch an anderen gesättigten Eisenstrecken auf, wie dem Joch oder dem Rotor. Näheres dazu findet sich in Abschnitt 5.2.2.1. Daher wird in dieser Arbeit überwiegend der allgemeine Begriff „Entlastung" verwendet.

Mit der entwickelten Auswertmethode, die in Abschnitt 4.2 vorgestellt wird, ist der Verlauf des Entlastungsfelds am Nutgrund in Abbildung 5-2 gut zu erkennen.

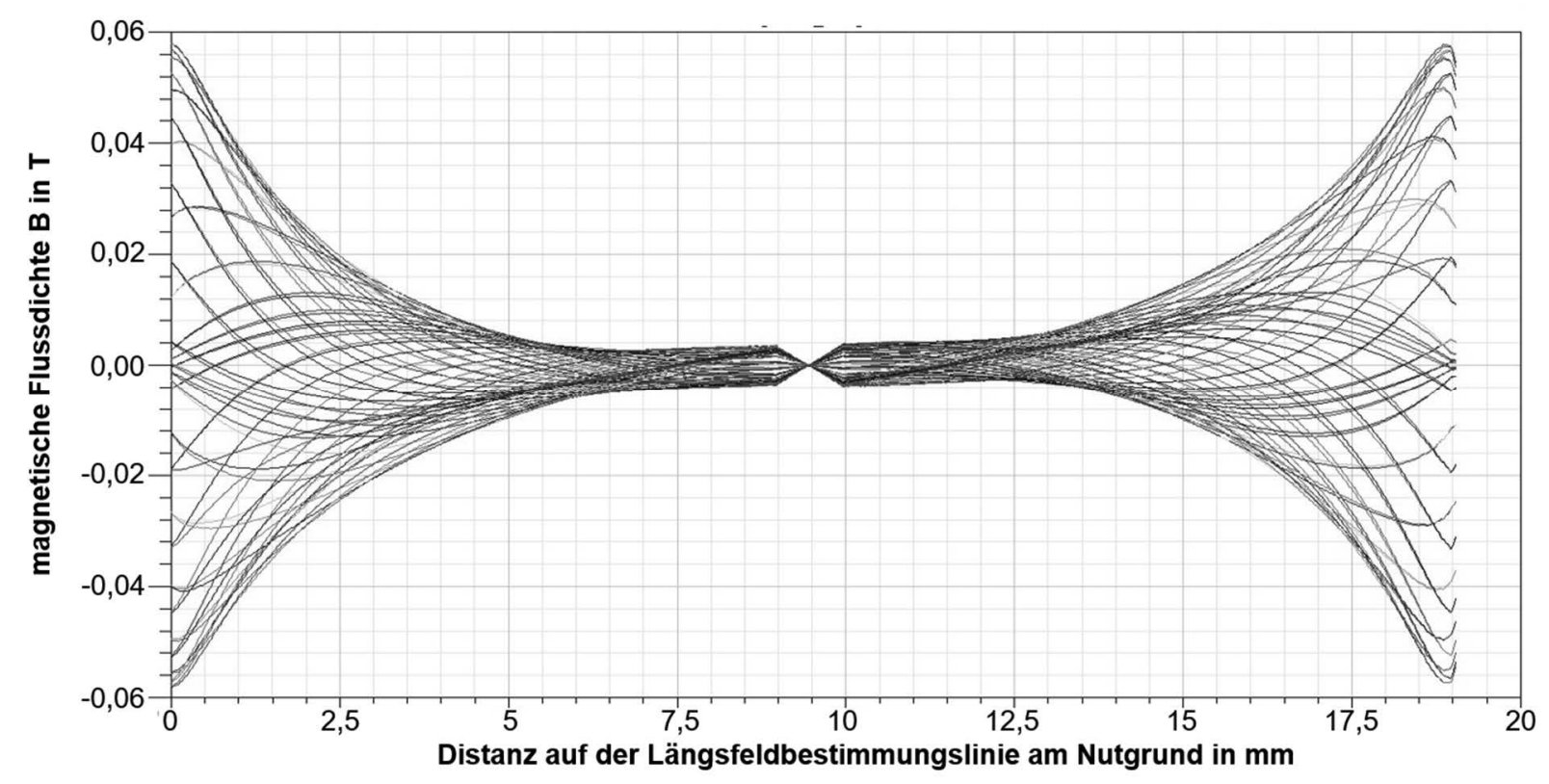

Abbildung 5-2: Magnetische Flussdichte in Längsrichtung am Nutgrund (vergleiche Abbildung 5-4) mit typischer Zahnentlastung durch magnetische Sättigung der Zähne bei einer Zahninduktion von 2,14 T

Das Entlastungsfeld erreicht die höchsten Werte in direkter Nähe der zu entlastenden Eisenstrecke. Dabei handelt es sich um die beiden gesättigten Zähne, die sich links und rechts in unmittelbarer Nähe des ausgewerteten Bereichs befinden. Mit zunehmender Entfernung von der Zahnflanke nimmt die Amplitude des Zahnentlastungsfelds ab - auch weil der umschlossene elektrische Strom geringer wird. Wie die gezeigte Abbildung zu interpretieren ist, kann in Abschnitt 4.2 nachgelesen werden.

Abbildung 5-3 zeigt ein weiteres Beispiel eines magnetischen Entlastungsfelds. 


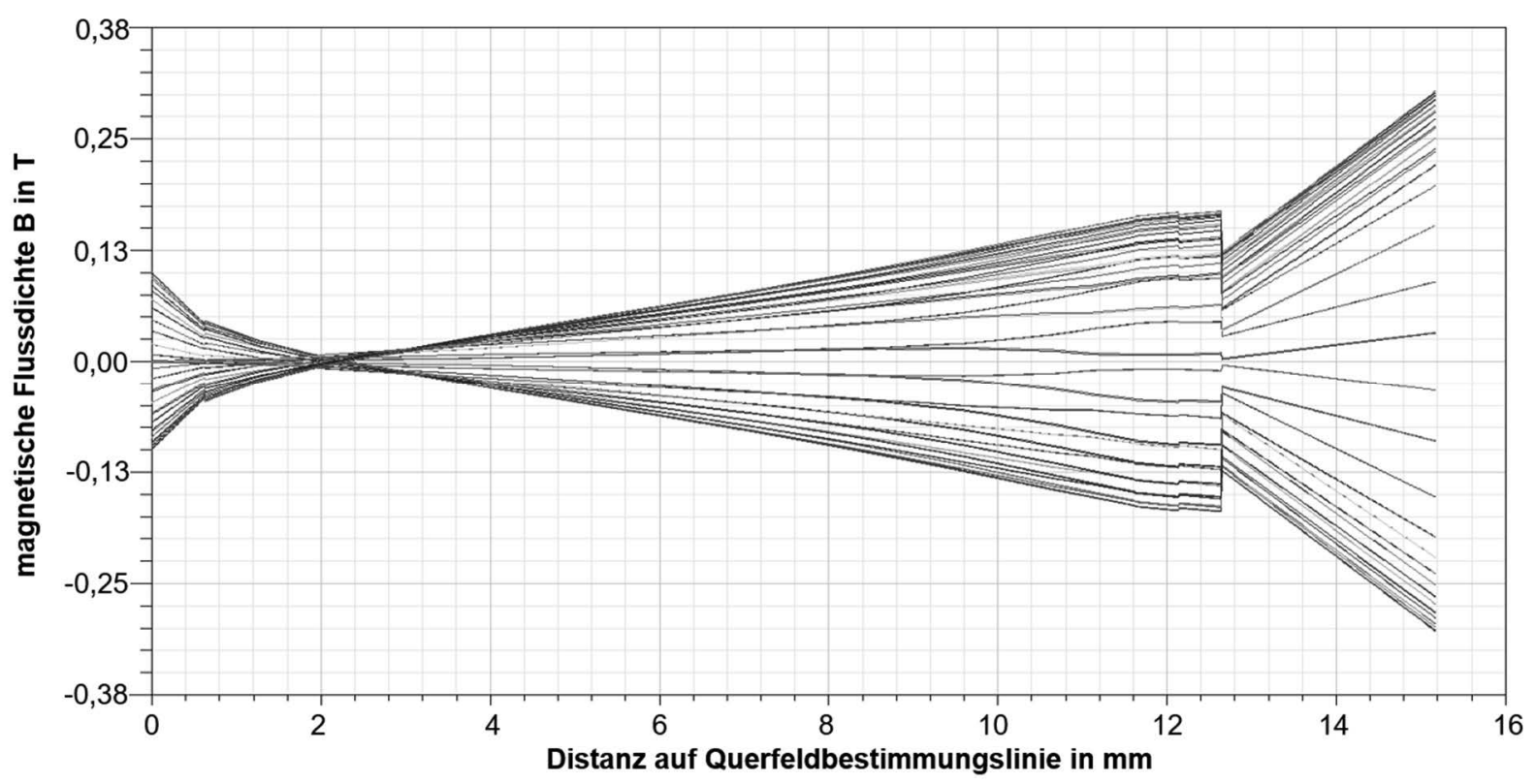

Abbildung 5-3: Magnetische Flussdichte in Querrichtung an der Zahnflanke (vergleiche Abbildung 5-4 und Abbildung 4-4) mit Jochentlastung bei magnetischer Sättigung des Jochs

Die magnetische Flussdichte in Querrichtung ist dargestellt. Im Vergleich zu Abbildung 4-4, die die magnetische Flussdichte in Querrichtung ohne magnetische Sättigung des Eisens zeigt, ist hier deutlich das Entlastungsfeld am Nutgrund zu erkennen, das sich in der Darstellung links befindet. Ein weiterer Unterschied zu Abbildung 4-4 findet sich rechts in der Darstellung. Hier sind eine Unstetigkeit im Querfeld und ein weiteres starkes Ansteigen des Querfeldes zu sehen. Dieser Unterschied ist damit zu erklären, dass die Linie für die Querfeldbestimmung länger ist als in Abbildung 4-3 und bis in den Nutraum oberhalb der Leiter hineinragt. Durch das Hinzufügen des Rotors oberhalb der Nut treten teilweise Feldlinien seitlich aus den Zähnen aus, die anschließend bogenförmig in den Rotor verlaufen. Dieser Umstand wird beispielsweise bei Richter [50] beschrieben.

Entlastungsfelder können die Stromverdrängung erhöhen, wenn sie den elektrischen Leiter durchdringen. Magnetische Sättigung in den Zähnen oder im Joch verursacht in der Regel solche Entlastungsfelder. Aber auch ein Entlastungsfeld am Rotor kann sich auf die Stromverdrängung auswirken, da durch dieses das Querfeld in der Nähe der Nutöffnung zunimmt.

Generell ist eine geringere Angriffsfläche des Leiters in Feldrichtung vorteilhaft, um die Anfälligkeit des Leiters für Stromverdrängung zu reduzieren. Beim Querfeld bedeutet dies eine niedrigere Leiterhöhe. Beim Entlastungsfeld hängt die Richtung von der Lage der entlasteten Eisenstrecke ab. Bei Entlastungen des Jochs oder des Rotors ist ebenfalls eine geringe Leiterhöhe von Vorteil, da diese Entlastungsfelder quer zur Nut verlaufen. Dagegen ist bei magnetischer Zahnentlastung eine geringe Leiterbreite nützlich, da dieses Entlastungsfeld längs der Nutrichtung verläuft. Im Übergangsbereich zwischen Joch und Stator treten jedoch bei magnetischer Sättigung in einem der beiden Abschnitte sowohl Längs- als auch Querfeldanteile auf (siehe Abschnitt 5.2.2.1). 


\subsection{Untersuchung anhand von Finite-Elemente-Rechnungen}

In diesem Abschnitt werden die in Abschnitt 5.1 beschriebenen Effekte mittels FiniteElemente-Rechnungen nachvollzogen. In Abschnitt 5.2.1 wird das einfache Grundmodell aus Abschnitt 4.2 herangezogen. Von diesem ist aus dem vorhergehenden Abgleich bekannt, dass es sich nach der fieldschen Theorie verhält, da ihm vergleichbare Randparameter zugrunde liegen. Das Modell wird nur leicht verändert, um den Einfluss der magnetischen Sättigung berücksichtigen und in Abschnitt 5.2.2 untersuchen zu können. In Abschnitt 5.2.3 wird sich dem Problem von einer anderen Seite genähert. Es wird das Finite-Elemente-Modell der aus Kapitel 3 bekannten elektrischen Maschine mit gegossenen Spulen herangezogen. Dabei wird geprüft, wie sich der Wegfall der magnetischen Sättigung auf die Stromverdrängung auswirkt.

\subsubsection{Modifikation des Grundmodells}

Das Modell für die Untersuchung des Einflusses der magnetischen Sättigung auf die Stromverdrängung ist nach der schematischen Abbildung 5-4 aufgebaut.

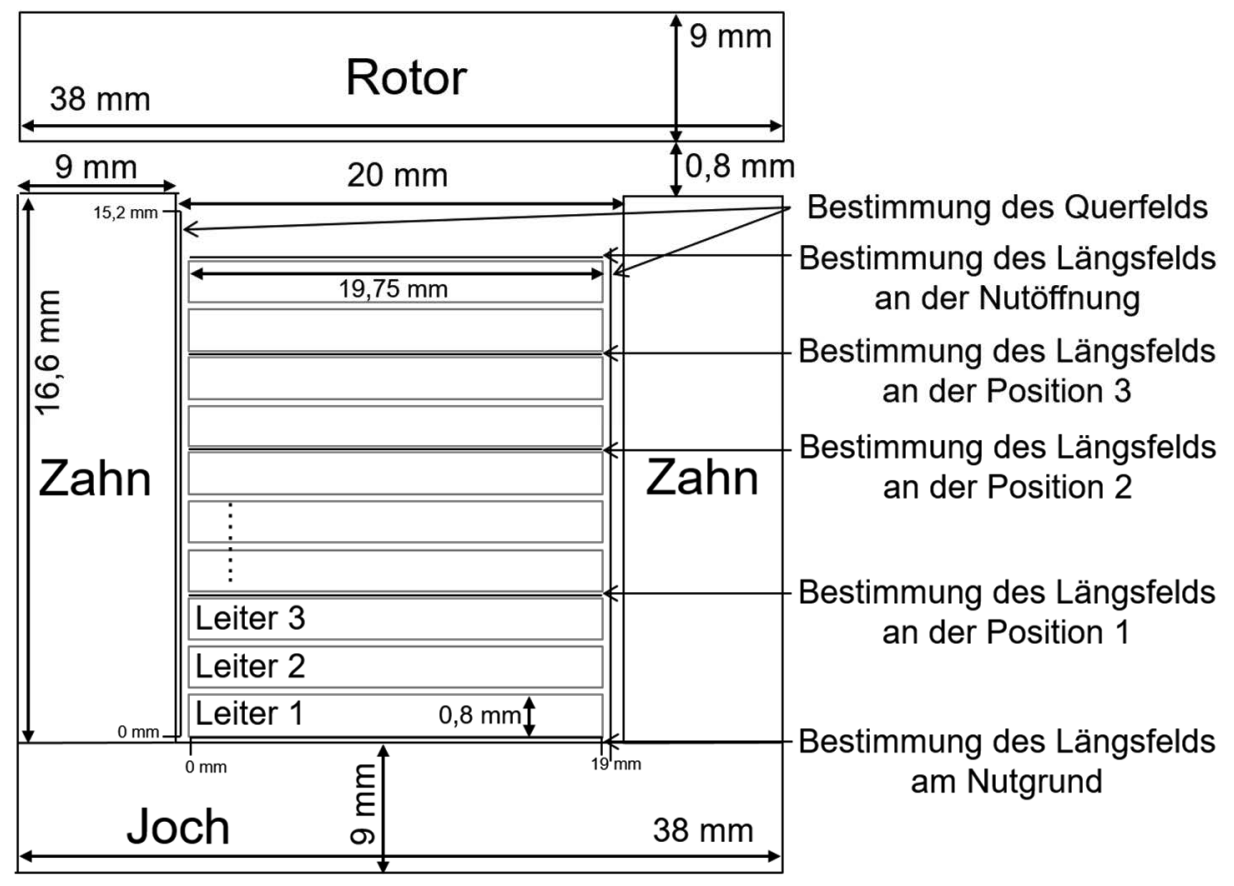

Abbildung 5-4: Erweitertes und angepasstes Finite-Elemente-Grundmodell

Der angedeutete Rotor ist für einen ausreichend geringen magnetischen Widerstand des Eisenkreises notwendig. Denn nur mit einem geringen magnetischen Widerstand ist die Erreichung hoher magnetischer Flussdichten in den Zähnen möglich, die für ein magnetisches Sättigungsverhalten erforderlich sind. Bei der Leitergeometrie werden wieder flache, breite Leiter mit einer geringen Anfälligkeit für das Nutquerfeld gewählt. Im Verlaufe der Untersuchung werden die unterschiedlichen Bereiche des Eisenkreises, bestehend aus Joch, Zähnen und Rotor, in die Sättigung getrieben. Die Bereiche sind, wie in Abbildung 5-4 gezeigt, voneinander abgegrenzt. Als Materialparameter für den Eisenkreis werden für magnetisch sättigende Bereiche die des handelsüblichen NO30-Blechs von C.D. Wälzholz verwendet. Für die restlichen Abschnitte des Eisenkreises, die magnetisch nicht sättigen sollen, wird ein theoretisches 
Material angenommen, das sich bis 1,91 T wie NO30-Blech von C.D. Wälzholz verhält und anschließend eine konstante relative Permeabilität von 17 beibehält. Die Simulationen werden bei einer Stromfrequenz von $933 \mathrm{~Hz}$ durchgeführt.

\subsubsection{Mit Finite-Elemente-Rechnungen durchgeführte Untersuchungen}

\subsubsection{Auswirkungen unterschiedlicher Sättigungsbereiche auf die Stromverdrängung}

Zunächst werden die unterschiedlichen Bereiche aus Abbildung 5-4 in verschiedenen Kombinationen gesättigt. Dies erfolgt bei einem Strom von 2000 A, um ein hohes $\mathrm{Maß}$ an Sättigung zu erreichen und dadurch die entstehenden Auswirkungen gut erkennen zu können.

......ohne Sättigung

- - Rotor, Joch gesättigt

๑๐Rotor, Zähne gesättigt

- - Zähne gesättigt
Rotor gesättigt

—Rotor, Joch, Zähne gesättigt

Ј Joch, Zähne gesättigt

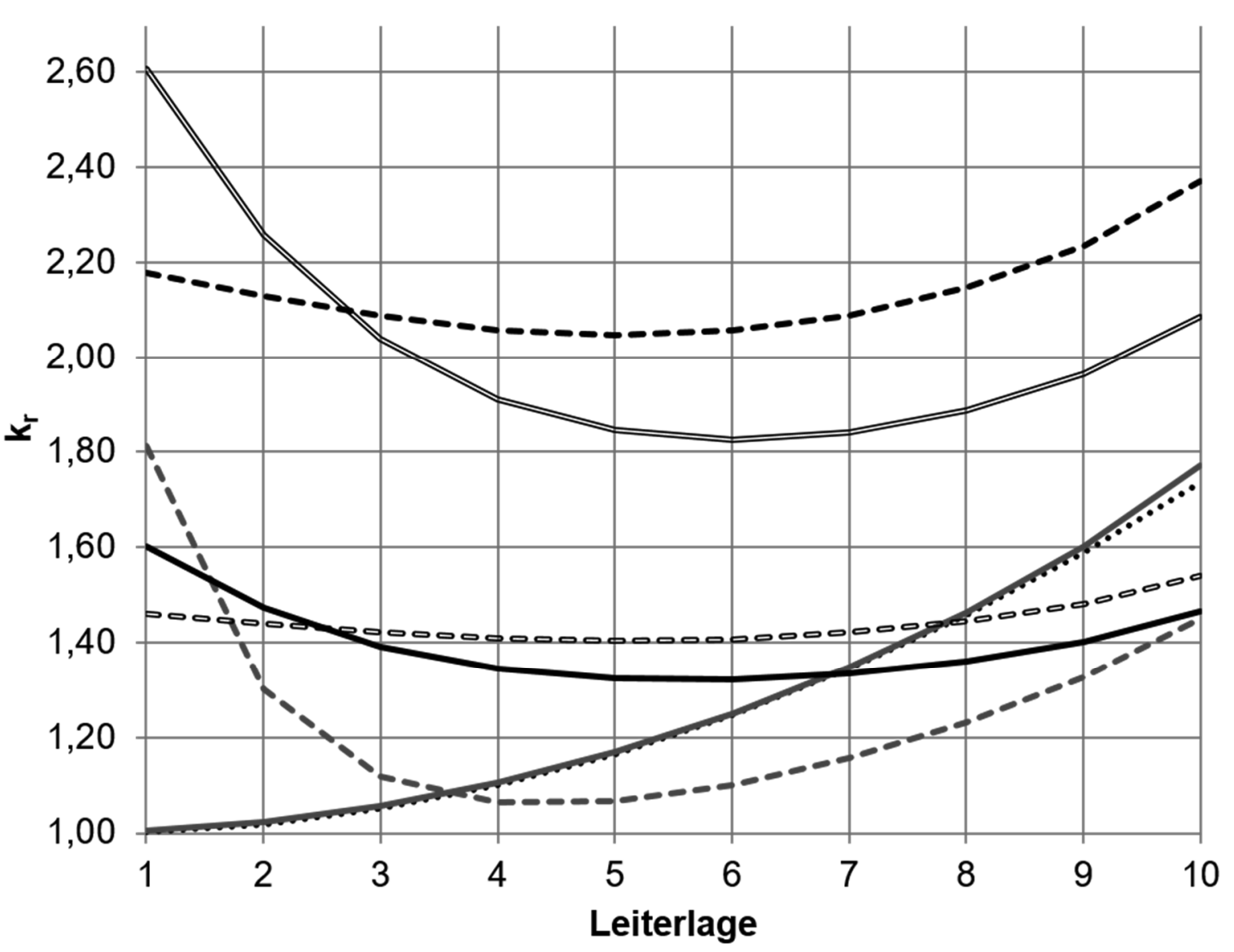

Abbildung 5-5: Stromverdrängungsverläufe bei magnetischer Sättigung $(B=2,67 \mathrm{~T})$ von unterschiedlichen Eisenkreisabschnitten 
In Abbildung 5-5 sind mehrere Sättigungsszenarien dargestellt. Gezeigt wird der Widerstandserhöhungsfaktor in den einzelnen Leiterlagen, beginnend mit dem untersten Leiter in der Nut gemäß Abbildung 4-3. Es ist zu erkennen, dass die Sättigung einen signifikanten Einfluss auf die Stromverdrängung hat. Die Sättigung unterschiedlicher Bereiche beziehungsweise Bereichskombinationen führt zu erkennbar unterschiedlichen Stromverdrängungsverläufen. Dabei unterscheiden sich die Stromverdrängungsverläufe sowohl in ihrer Form als auch in ihrer Lage. Ein alleiniges Sättigen des Rotors unterscheidet sich kaum von der ungesättigten Variante. Dies lässt sich durch die Vergleichbarkeit zu einer Variante ohne magnetischen Rückschluss erklären.

Je mehr Teile des Eisenkreises gesättigt sind, desto tiefer sinkt tendenziell das $\mathrm{Ni}$ veau des Widerstandserhöhungsfaktorverlaufs. Hier spielen der höhere magnetische Widerstand gegenüber einem nicht sättigenden Eisenkreis und die in Abschnitt 5.1.1 beschriebene, damit einhergehende Verminderung der Querfeldkomponente eine entscheidende Rolle. Eine Ausnahme bildet dabei die Variante, bei der Rotor und Joch gesättigt sind. Diese erreicht ab der vierten Leiterlage die geringsten Widerstandserhöhungsfaktoren, obwohl weniger Eisenkreisabschnitte gesättigt sind als bei der Variante mit gesättigtem Rotor, gesättigtem Joch und gesättigten Zähnen. Die Ursache hierfür ist, dass sich durch die fehlende Zahnentlastung keine zusätzliche Längsfeldkomponente ausbildet. Die betrachteten Leiter sind durch ihre Breite sehr anfällig für magnetische Längsfelder und durch die geringe Höhe wenig anfällig für Querfeldanteile. Der Wegfall der Längsfeldkomponente reduziert die Stromverdrängung signifikant. Durch das gesättigte Joch ist die Stromverdrängung am Nutgrund bei der Variante mit gesättigtem Joch und gesättigtem Rotor verhältnismäßig hoch. Der Grund hierfür ist, wie in Abschnitt 5.1.2 erklärt wurde, dass sich bei sättigendem Joch oder sättigenden Zähnen am Übergang der beiden Bereiche sowohl Längsfeldals auch Querfeldanteile herausbilden. Denn die Feldlinien verlaufen vom Zahnentlastungsfeld ins Joch oder vom Jochentlastungsfeld in den Zahn. Dadurch ergibt sich bei der Variante mit sättigendem Rotor und sättigendem Joch am Nutgrund ein hoher Längsfeldanteil, der sich durch zusätzliche Stromverdrängung in diesem Bereich bemerkbar macht.

Die Varianten mit nicht sättigendem Rotor erreichen die höchsten Stromverdrängungswerte. Die Ursache ist, dass die Feldlinien in den nicht sättigenden Rotor streben und sich dadurch ein um den Faktor drei bis sechs stärkeres Längsfeld an der Nutöffnung ausbildet. Interessant ist auch, dass die Varianten "Zähne gesättigt" und „Joch und Zähne gesättigt" im Verlauf und in der Lage zueinander den Varianten „Rotor und Zähne gesättigt" und „Rotor, Joch und Zähne gesättigt" ähneln. Der Unterschied ist auf das ausgeprägte Längsfeld der Varianten mit nicht gesättigtem Rotor sowie dem geringeren magnetischen Widerstand ihrer Eisenkreise zurückzuführen.

In Tabelle 4 sind die maximalen magnetischen Flussdichten der verschiedenen Sättigungsvarianten zusammengefasst. 


\begin{tabular}{|c|c|c|c|c|c|c|}
\hline Querfeld in T & 0,35 & 0,32 & 0,55 & 0,52 & 0,96 & 0,75 \\
\hline $\begin{array}{l}\text { Querfeld } \\
\text { unten in T }\end{array}$ & 0,04 & 0,58 & 0,03 & 0,38 & 0 & 1,4 \\
\hline Nutgrund in $T$ & 0,5 & 0,65 & 0,33 & 0,42 & 0,02 & 0,46 \\
\hline $1 \mathrm{in} \mathrm{T}$ & 0,54 & 0,47 & 0,34 & 0,3 & 0,04 & 0,07 \\
\hline 2 in $T$ & 0,52 & 0,46 & 0,3 & 0,27 & 0,04 & 0,05 \\
\hline 3 in T & 0,47 & 0,42 & 0,22 & 0,19 & 0,05 & 0,04 \\
\hline $\begin{array}{c}\text { Nutöffnung in } \\
T\end{array}$ & 0,36 & 0,3 & 0,11 & 0,1 & 0,09 & 0,06 \\
\hline mittleres $k_{r}$ & 2,14 & 2,03 & 1,44 & 1,4 & 1,28 & 1,26 \\
\hline
\end{tabular}

Tabelle 4: Übersicht über maximal erreichte Flussdichten im Nutraum und mittlere Widerstandserhöhungsfaktoren $k_{r}$ von unterschiedlich gesättigten Eisenkreisen

Die Zeile "Querfeld" gibt die maximale magnetische Flussdichte in Querrichtung in der obersten Leiterlage an. Der Wert der Zeile "Querfeld unten" gibt die maximale magnetische Flussdichte in Querrichtung in der untersten Leiterlage an. Das "Querfeld unten" entsteht nur wenn eine Entlastung der Zähne oder des Jochs vorliegt. Daher ist der Wert im Falle eines nicht sättigenden Jochs in Kombination mit nicht sättigenden Zähnen gleich 0 . Die Varianten sind nach dem mittleren $k_{r}$-Wert sortiert, der als letzte Zeile in der Tabelle aufgetragen ist. Dabei handelt es sich um den über alle Leiterlagen gemittelten Widerstandserhöhungsfaktor. Er gibt keine Auskunft darüber, wo in der Spule zusätzliche Verluste auftreten, aber er gibt komprimiert in einem Wert an, wie stark die Spule von Stromverdrängung betroffen ist. Wie zu sehen ist, steigt der mittlere $k_{r}$-Wert bei der untersuchten Geometrie mit den Längsfeldanteilen im oberen Nutbereich an. Dadurch haben die Varianten mit gesättigten Zähnen, bei denen sich ein Entlastungsfeld in Längsrichtung ausbildet, höhere mittlere Widerstandserhöhungsfaktoren. Ein gesättigter Rotor erhöht den Querfeldanteil an der Nutöffnung durch ein Entlastungsfeld. Ein nicht sättigender Rotor führt durch den geringeren magnetischen Widerstand zu einem höheren Niveau der anderen auftretenden Entlastungsfelder.

Die Anfälligkeit der betrachteten Geometrie für Längsfeldanteile lässt sich anhand der Varianten mit dem höchsten und dem niedrigsten mittleren $k_{\text {- }}$-Wert erkennen. Die höchsten Stromverdrängungsverluste hat die Variante, bei der lediglich die Zähne sättigen, wodurch ausschließlich ein Entlastungsfeld in Längsrichtung entsteht. Den geringsten Widerstandserhöhungsfaktor hat nicht die Variante, bei der der gesamte Eisenkreis sättigt. Schließlich erfährt diese durch die magnetische Sättigung die stärkste Verminderung der Streufeldanteile gemäß Abschnitt 5.1.1. Der niedrigste mittlere $k_{r}$-Wert wird bei der Variante mit sättigendem Rotor und sättigendem Joch erreicht. Denn bei ihr vermindert sich die magnetische Flussdichte durch Sättigung, und gleichzeitig bilden sich die Entlastungsfelder nur quer zur Nut aus. Nur diese Va- 
riante erreicht einen geringeren mittleren Widerstandserhöhungsfaktor als die nicht sättigende Variante, bei der der mittlere $k_{r}$-Wert 1,27 beträgt.

Es lässt sich zusammenfassen, dass die Auswirkungen der magnetischen Sättigung des Eisenkreises vom jeweils sättigenden Abschnitt und von der Leitergeometrie abhängen. Die in Abschnitt 5.1.1 und 5.1.2 beschriebenen Effekte lassen sich anhand der Finite-Elemente-Simulationsergebnisse nachvollziehen. Dabei hängt die Richtung des Entlastungsfelds von der Richtung der entlasteten Eisenstrecke ab. Die hier vorliegende Leitergeometrie ist anfällig für Längsfelder und erreicht daher bei Zahnsättigung den höchsten mittleren Widerstandserhöhungsfaktor.

\subsubsection{Auswirkung zunehmender Sättigung auf das Nutfeld und die Stromverdrängung}

Im nächsten Schritt soll ein Verständnis für die nichtlinearen Zusammenhänge der magnetischen Sättigung entwickelt werden. Dazu wird die Variante mit magnetischer Sättigung der Zähne und des Jochs bei unterschiedlichen Strömen betrachtet. Mit steigendem Strom in der Maschine werden die Zähne und das Joch zunehmend in Sättigung gebracht. Dabei wird zunächst die Auswirkung auf das magnetische Feld in der Nut studiert. Dieses stellt die Ursache der resultierenden Stromverdrängung dar.

Abbildung 5-6 zeigt den Verlauf der magnetischen Flussdichte in Abhängigkeit vom Strom.

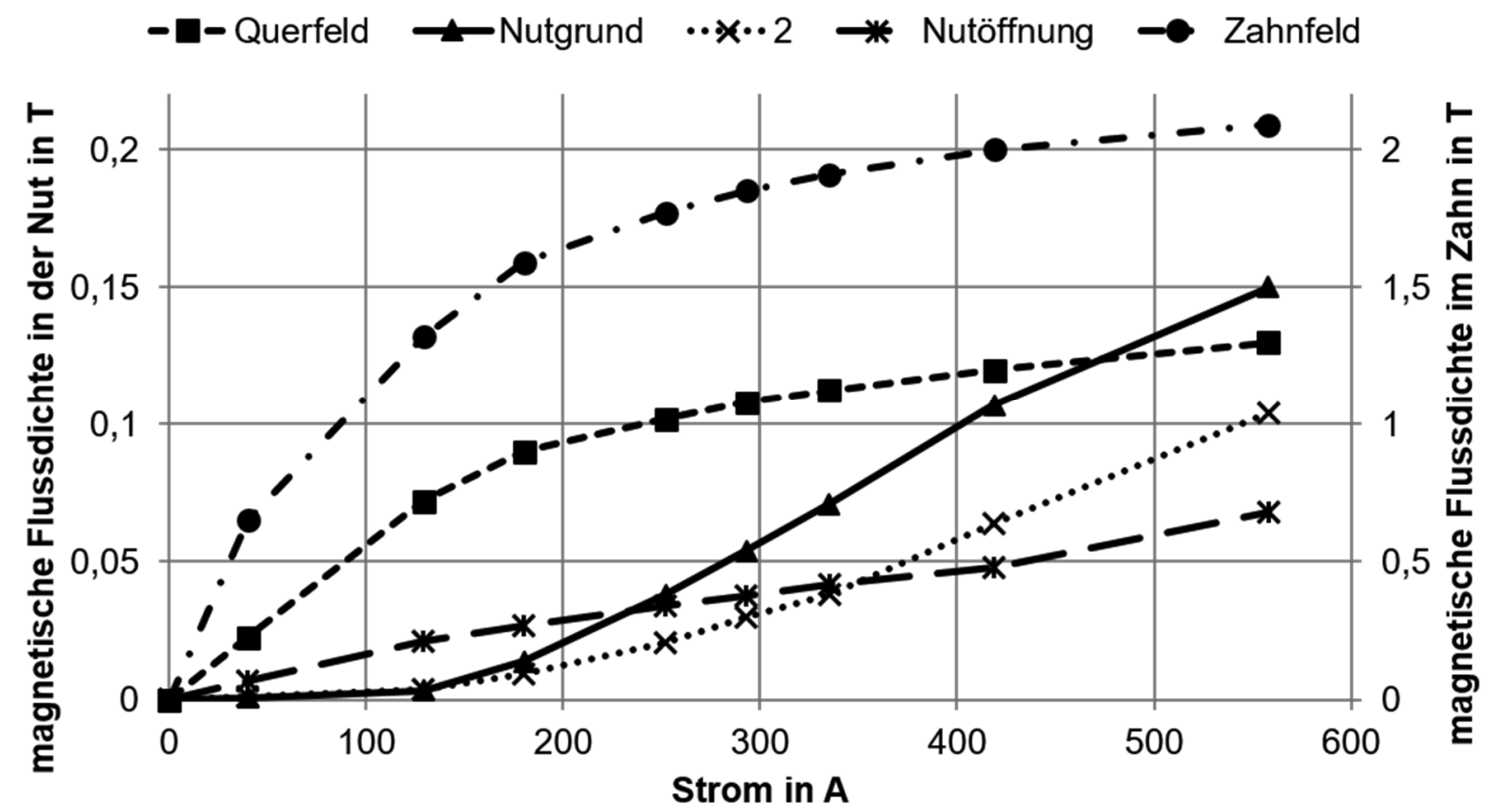

Abbildung 5-6: Auswirkung der magnetischen Sättigung auf die magnetische Flussdichteverteilung in der Nut und im Zahn

Dabei ist auf der rechten y-Achse als Orientierungshilfe der Verlauf der magnetischen Flussdichte im Zahn dargestellt. Auf der linken y-Achse ist die Flussdichte der Streufeldanteile "Querfeld" sowie der Längsfeldanteile "Nutgrund", „2" und "Nutöffnung" aufgetragen. Eine räumliche Zuordnung der Streufeldanteile ist mit Hilfe der Abbildung 5-4 oder der Beschreibung in Abschnitt 4.3.1 möglich. Von den Streuanteilen ist jeweils der maximal erreichte Wert auf der ausgewerteten Strecke angegeben. Bei der magnetischen Flussdichte in Querrichtung liegt dieser nahe an der Nutöffnung. 
Dort erreicht das reguläre Nutquerfeld seine höchsten Werte, wie es den fieldschen Betrachtungen [11] zugrunde liegt. Bei den magnetischen Flussdichteanteilen in Längsrichtung erreichen die Bereiche nahe an den Zähnen die höchsten Werte. Dabei handelt es sich um das Zahnentlastungsfeld. An den Verläufen in Abbildung 5-6 ist gut zu erkennen, dass die magnetischen Streuflussdichten einen Bruchteil der Flussdichte im Zahn erreichen - hier sind es unter 10\%. Des Weiteren zeigt die Darstellung, dass das reguläre Nutquerfeld proportional zum Zahnfeld von der Sättigung betroffen ist. Soweit handelt es sich um keine unerwarteten Erkenntnisse. Interessant ist die gleichzeitige Betrachtung der Längskomponente. Das Zahnentlastungsfeld ist bis zum Eintritt in die Sättigung nicht vorhanden, dann beginnt es annährend linear zu steigen und übersteigt am Nutgrund bei rund 460 A die Amplitude des Nutquerfelds.

Der Verlauf der Längsfeldkomponente "2" ähnelt dem Verlauf am Nutgrund - allerdings mit einer geringeren Steigung. Eine mögliche Erklärung bietet Gleichung (2-15). In diesem Bereich der Nut fällt möglicherweise der magnetische Widerstand höher aus, da längere Teile des magnetischen Kreises im Nutraum liegen. In der Folge fiele die magnetische Flussdichte geringer aus.

An der Nutöffnung sind die Verhältnisse anders. Die magnetische Flussdichte in Längsrichtung an der Nutöffnung ähnelt in ihrem Verlauf dem Verlauf der magnetischen Flussdichte in Querrichtung in abgeschwächter Form. Sie steigt ebenfalls vom Ursprung aus an und verringert die Steigung mit dem Eintritt in den Sättigungsbereich. Eine Erklärung hierfür ist, dass es sich um keine Entlastungserscheinung handelt. Dies lässt sich gut am Verlauf der magnetischen Flussdichte in Abbildung 5-7 erkennen.

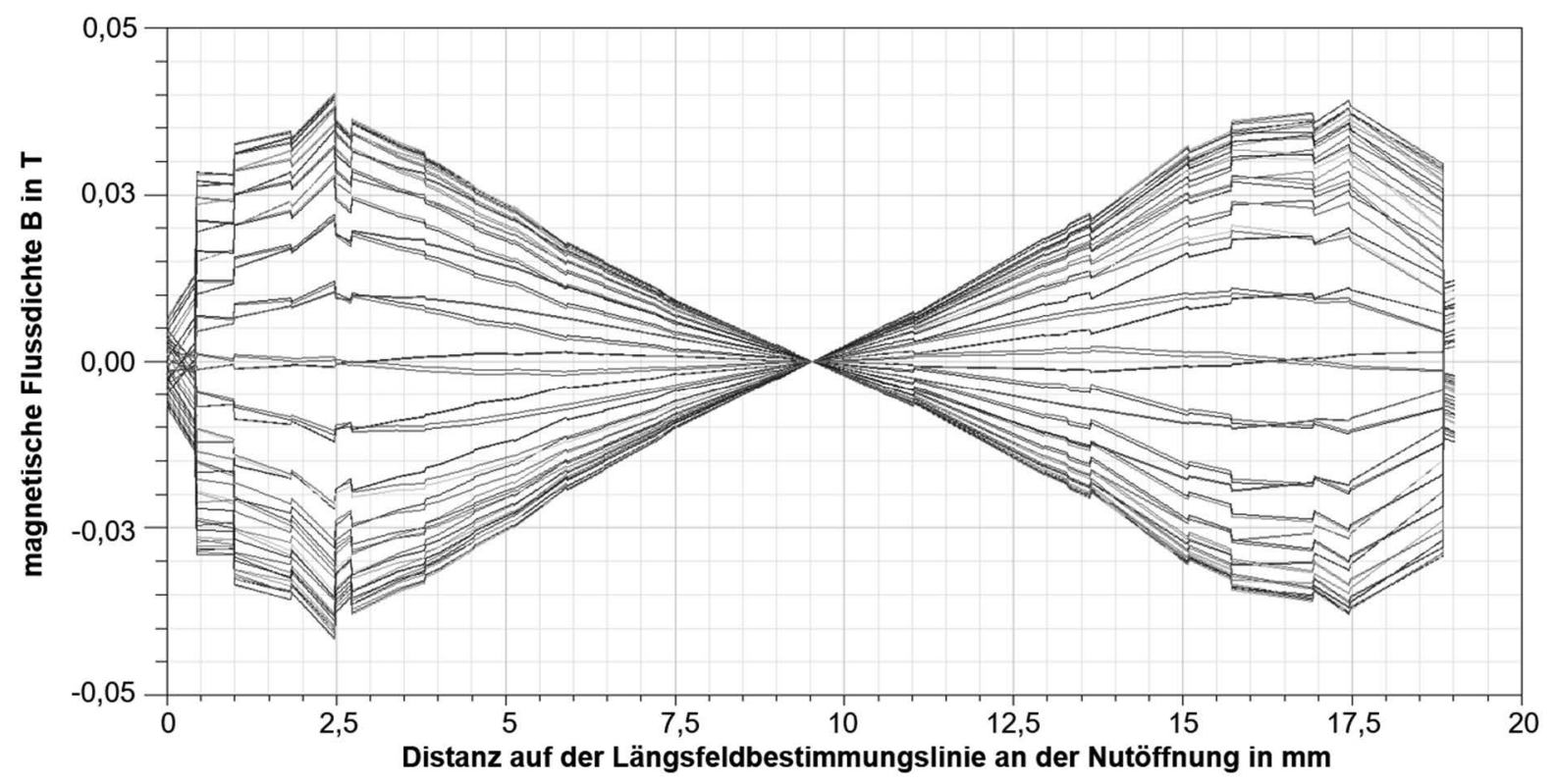

Abbildung 5-7: Magnetische Flussdichteverteilung in Längsrichtung an der Nutöffnung

Anders als in Abbildung 5-2 befindet sich das Maximum des Flussdichteverlaufs nicht unmittelbar an den Zahnflanken. Es handelt sich vielmehr um eine Ablenkung der Feldlinien, die durch den Rotor verursacht wird. Dieser Effekt wird bereits bei Richter [27] beschrieben. 
In Abbildung 5-8 ist der Widerstandserhöhungsfaktor für die einzelnen Leiterlagen bei verschiedenen Strömen zu sehen.

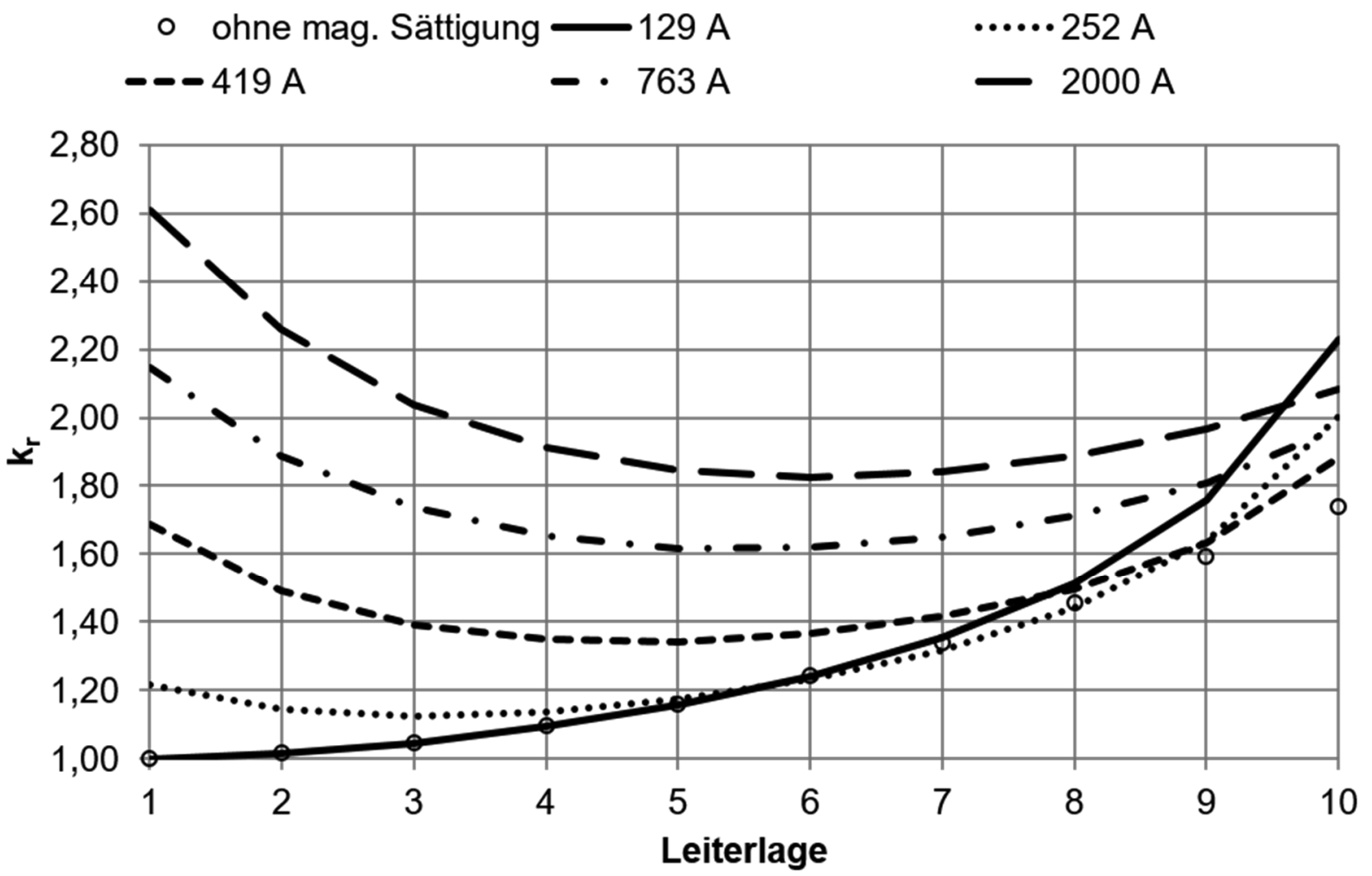

Abbildung 5-8: Verlauf des Widerstandserhöhungsfaktors über die Leiterlagen für unterschiedlich hohe Ströme in den Leitern

Es fällt zunächst auf, dass der Widerstandserhöhungsfaktor in den drei oberen Leiterlagen bei niedrigen Strömen höher ist, als es die analytische Bestimmung auf Basis des Nutquerfelds vermuten lässt. Ursache sind seitlich aus den Zähnen austretende Feldlinien, die in den Rotor verlaufen. Sie werden beispielsweise bei Richter [50] behandelt. Diese Feldlinien durchqueren die oberen Leiter in der Nut teilweise in Längsrichtung, wofür die breite, flache Leitergeometrie anfällig ist. Infolgedessen steigen die Stromverdrängungsverluste in diesem Bereich an.

Der Widerstandserhöhungsfaktor nimmt mit steigendem Strom und einhergehendem Übergang in den Sättigungsbereich zu. Der größte Anstieg ist dabei am Nutgrund zu verzeichnen, da hier sowohl das Zahn- als auch das Jochentlastungsfeld wirken. In den mittleren Leiterlagen steigt der $k_{r}$-Faktor weniger stark an. In den oberen Leiterlagen dominiert die Verminderung des magnetischen Felds durch die Sättigung des Eisenkreises, wodurch sich der Widerstandserhöhungsfaktor in diesem Bereich reduziert. Anhand der $k_{r}$-Verläufe für unterschiedliche Ströme ist analog zu Abbildung 5-5 zu erkennen, dass die Sättigung des Eisenkreises einen signifikanten Einfluss auf die Stromverdrängung hat. Bei 419 A wird in den Zähnen des betrachteten Eisenkreises eine magnetische Flussdichte von rund 1,9 T erreicht.

\subsubsection{Auswirkung der magnetischen Sättigung auf den Verlauf des mittleren Widerstandserhöhungsfaktors}

Der Einfluss der magnetischen Sättigung auf die magnetischen Streuflüsse und die Stromverdrängung führt dazu, dass der Widerstandserhöhungsfaktor nicht mehr als 
unabhängig vom Strom betrachtet werden kann. In Gleichung (2-18) sind Ströme enthalten, die sich aber herauskürzen, wenn in allen Leitern derselbe Strom fließt. Daher sagt der gängige, in Abschnitt 2.1.4 beschriebene, analytische Ansatz zur Berücksichtigung der Stromverdrängung durch das Nutquerfeld ein von der Stromstärke unabhängiges $k_{r}$ voraus. Dies trifft im Fall eines nicht sättigenden Eisenkreises auch zu, wie in Abschnitt 4.3.2 gezeigt wurde. Für einen sättigenden Eisenkreis gilt dies nicht mehr, wie aus Abbildung 5-9 hervorgeht.

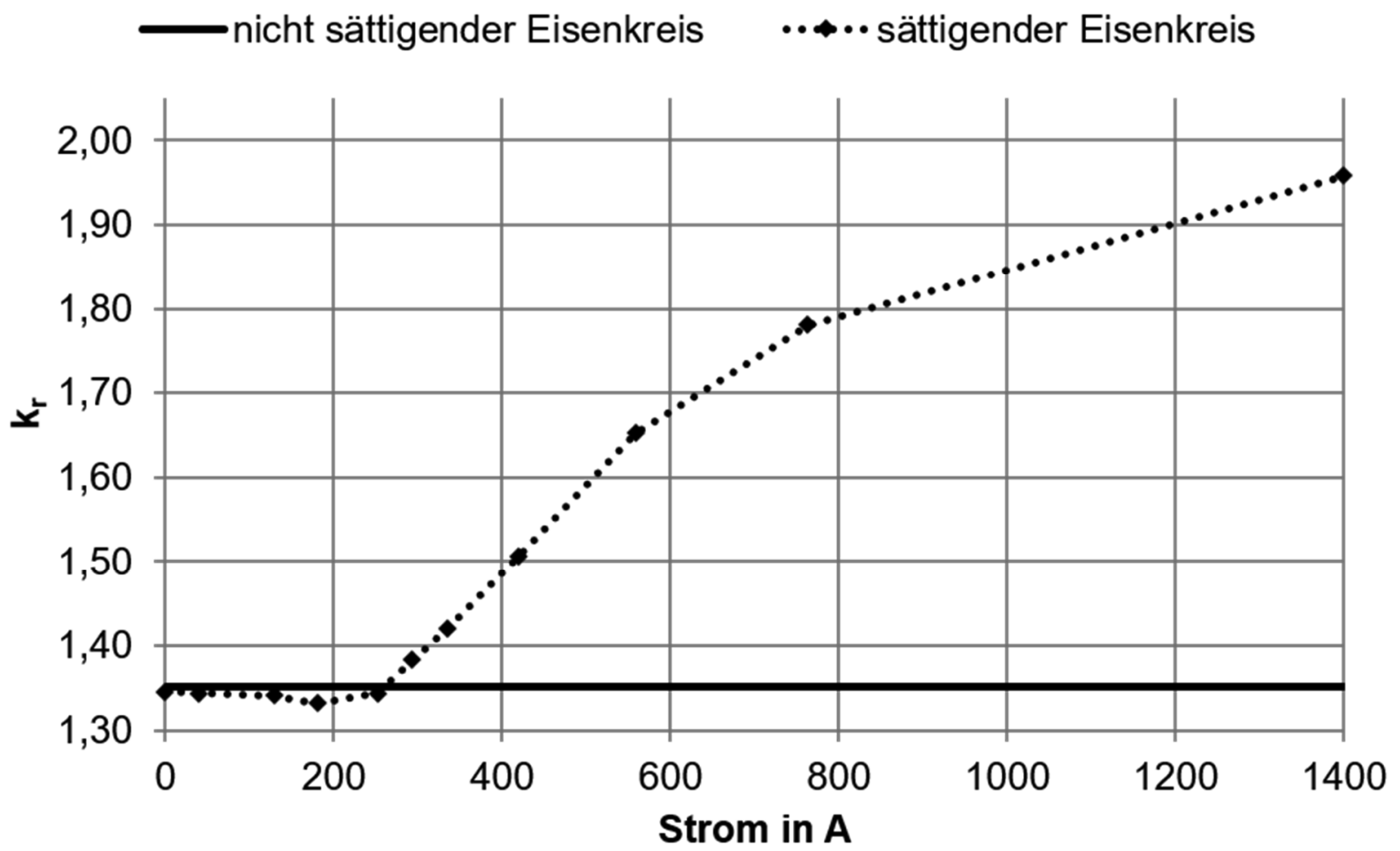

Abbildung 5-9: Anstieg des mittleren Widerstandserhöhungsfaktors aufgrund der zunehmenden Sättigung des Eisenkreises

Dort ist zu sehen, wie der Widerstandserhöhungsfaktor von der Sättigung beeinflusst wird. Bis zu einem Strom von rund 180 A fällt der Widerstandserhöhungsfaktor zunächst leicht $a b$, um anschließend stark anzusteigen. Die Steigung der $k_{r}$-Werte nimmt im weiteren Verlauf stetig ab. Der in Abbildung 5-9 gezeigte Verlauf soll anhand der Abbildung 5-10 erklärt werden. 


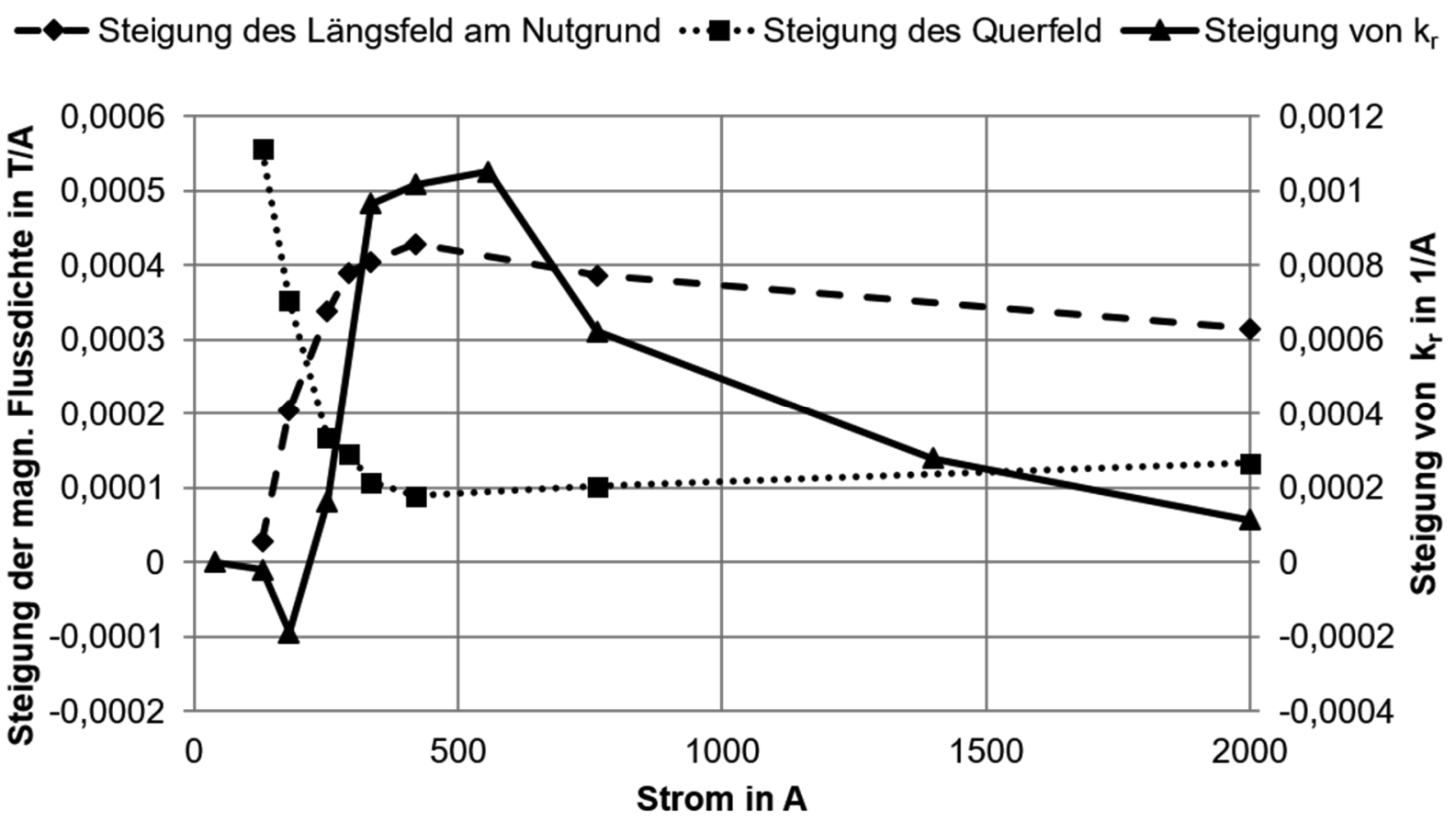

Abbildung 5-10: Betrachtung der Steigung von magnetischer Flussdichte in Längs- und Querrichtung als Ursache des sich mit dem Strom ändernden mittleren $k_{r}$-Werts

Dort ist auf der x-Achse der Strom aufgetragen, während auf der linken y-Achse die Steigung der magnetischen Flussdichte der Streuflüsse "Längsfeld am Nutgrund" und "Querfeld" dargestellt ist. Die rechte y-Achse zeigt die Steigung des Widerstandserhöhungsfaktors. Der Widerstandserhöhungsfaktor fällt bis zu einem Strom von $180 \mathrm{~A}$ leicht ab. Dies liegt in dem Bereich, in dem die Steigung des Querfelds stark abnimmt, während die Steigung des Längsfelds ansteigt. Sobald das Längsfeld eine hohe Steigung erreicht, wächst auch der Widerstandserhöhungsfaktor stark an. Die Steigung schwächt sich an dem Punkt ab, an dem sich auch die Steigung des Längsfelds leicht reduziert. Es scheint, dass die Ursache für den starken Anstieg des Widerstandserhöhungsfaktors das wachsende Längsfeld ist.

\subsection{Einfluss der Leitergeometrie auf die Auswirkung der magnetischen Sättigung auf den Verlauf des mittleren Widerstandserhöhungsfaktors}

Es ist anzunehmen, dass der in Abbildung 5-9 gezeigte Verlauf des Widerstandserhöhungsfaktors von der Leitergeometrie beeinflusst wird, da diese die Anfälligkeit des Leiters für Längs- und Querfelder bedingt. Um diese These zu überprüfen, wird der Fall betrachtet, dass die verwendeten Leiter hochkant in der Nut liegen, wie in Abbildung 5-11 dargestellt. Dabei wird nach Tabelle 4 von Variante 1 ausgegangen, bei der nur die Zähne magnetisch sättigen. 


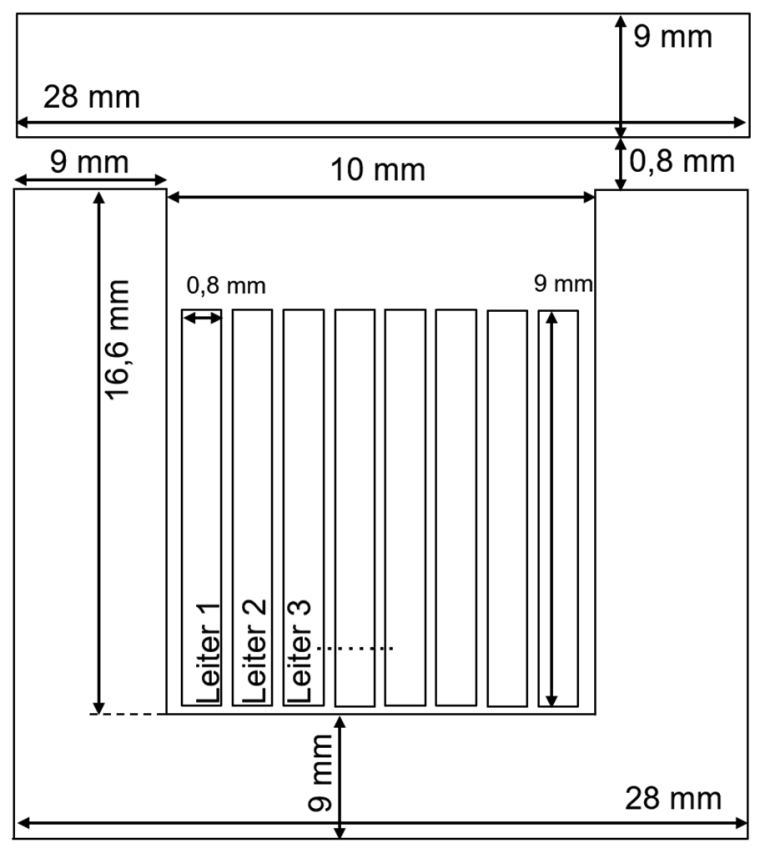

Abbildung 5-11: Hochkantleiteranordnung

Die Hochkantleiteranordnung ist anfällig für Querfeldanteile und wenig beeinflussbar durch Längsfeldanteile. Die Variante müsste bei niedrigen Strömen einen hohen Widerstandserhöhungsfaktor aufweisen. Bei hohen Strömen und starker Sättigung des Eisenkreises sollte die Leiteranordnung von der Verminderung der Querfeldkomponente profitieren und gleichzeitig nur wenig vom Zahnentlastungsfeld betroffen sein. In Abbildung 5-12 sind die Verläufe des Widerstandserhöhungsfaktors über die Leiterlagen für die Varianten mit Hochkantleitern und querliegenden Leitern gegenübergestellt.

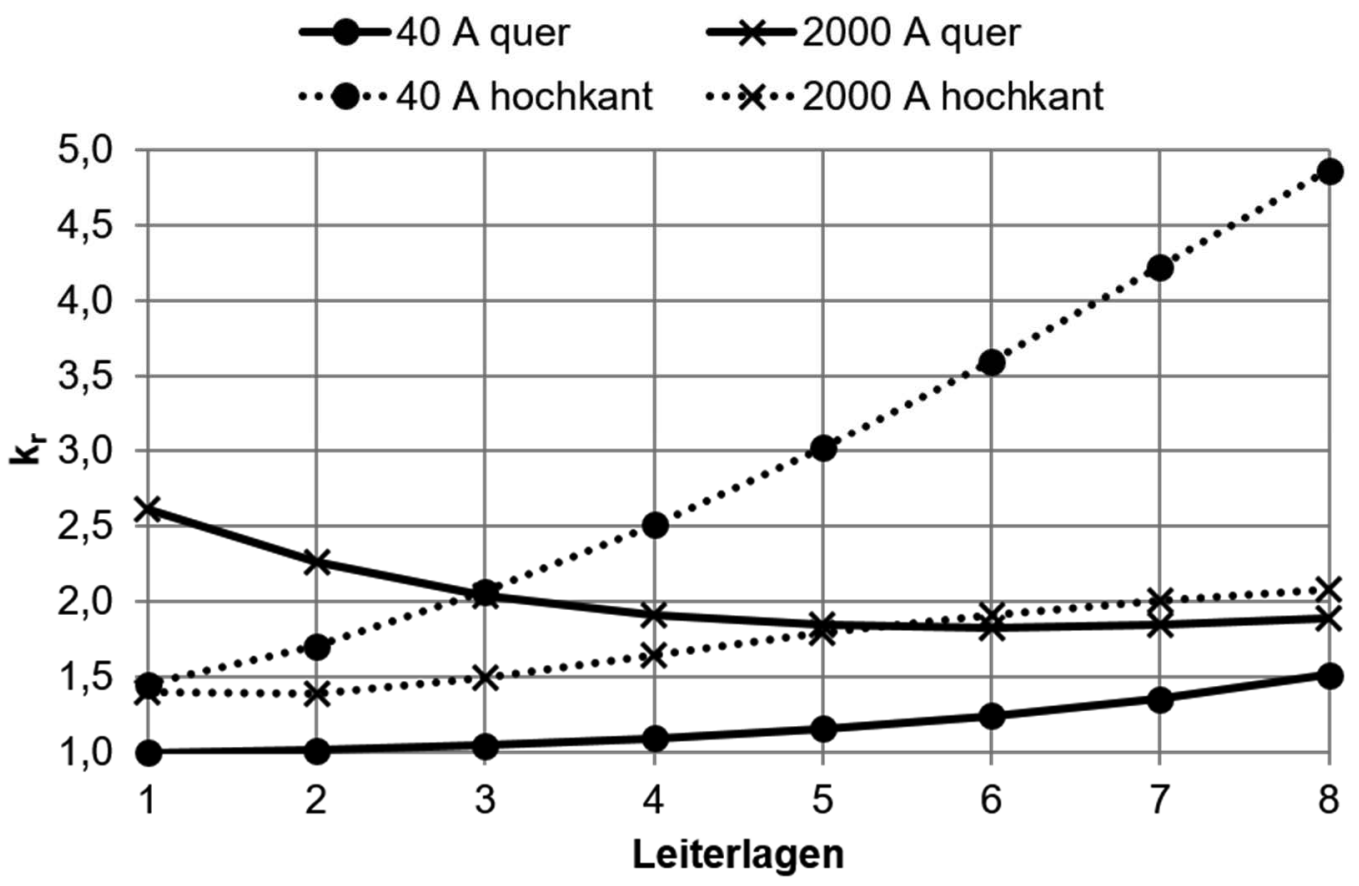

Abbildung 5-12: Gegenüberstellung der $k_{r}$-Verläufe bei hochkant- und bei querliegender Ausrichtung der Leiter bei unterschiedlicher magnetischer Sättigung des Eisenkreises 
Beide Varianten sind je bei einem Strom von $40 \mathrm{~A}$ und von $2000 \mathrm{~A}$ dargestellt, um zu sehen, wie sie durch die Auswirkungen der Sättigung beeinflusst werden. Bei $40 \mathrm{~A}$ liegt die Zahninduktion bei 0,65 T und bei $2000 \mathrm{~A}$ beträgt sie 2,67 T. Es ist deutlich zu erkennen, dass die Variante mit Hochkantleitern bei niedrigem Strom stärker von Stromverdrängung betroffen ist. Im Sättigungsfall entsprechen die Verhältnisse der aufgestellten These. Aufgrund der Verminderung der Querfeldkomponente und der Entstehung einer Längsfeldkomponente durch das Zahnentlastungsfeld nimmt bei Sättigung das Längsfeld in der Nut zu. Nun näheren sich die $k_{r}$-Verläufe von Hochkantanordnung und flach in der Nut liegenden Leitern an. Bei den Hochkantleitern kam es bei der Simulation des 40 A Betriebspunktes zu einer seitlichen Verschiebung des magnetischen Rückschlusses. Dadurch steigt der $k_{r}$-Verlauf zur rechten Seite hin stark an. Dieser Einfluss schränkt die Aussagekraft der Ergebnisse beträchtlich ein. Eine Tendenz lässt sich dennoch ablesen.

Abbildung 5-13 stellt die Verläufe der mittleren $k_{r}$-Werte der beiden Varianten bei verschiedenen Strömen einander gegenüber.

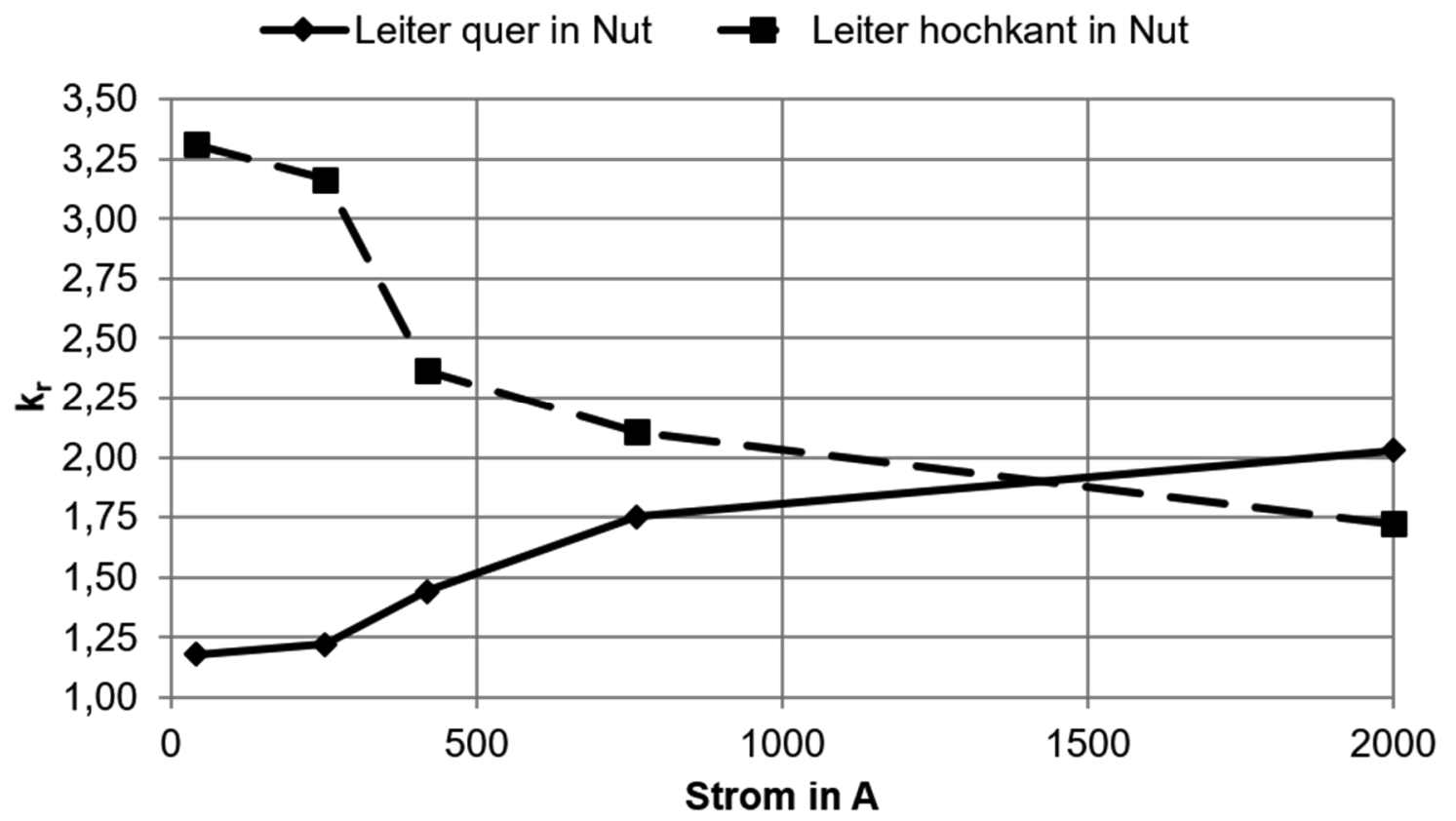

Abbildung 5-13: Gegenüberstellung des mittleren $k_{r}$-Verlaufs für hochkantund querliegende Ausrichtung der Leiter bei unterschiedlicher magnetischer Sättigung des Eisenkreises

Durch die Darstellung in Abhängigkeit vom Strom wird ein weiterer wichtiger Aspekt sichtbar: Der Einfluss der magnetischen Sättigung auf die Stromverdrängung ist bei Eintritt in den Sättigungsbereich am größten. Bei einem Strom von 419 A haben sich die mittleren Widerstandserhöhungsfaktoren der beiden betrachteten Leiteranordnungsvarianten bereits deutlich angenähert. Bei diesem Strom wird in den Zähnen des untersuchten Modells eine magnetische Flussdichte von rund 1,9 T erreicht, die für hoch ausgenutzte Maschinen vorstellbar ist. Somit ist die Berücksichtigung der magnetischen Sättigung für die Stromverdrängung in hoch ausgenutzten Maschinen erforderlich, da ihr Einfluss bereits signifikant ist.

Zusammenfassend lässt sich für den Abschnitt 5.2.2 sagen, dass die magnetische Sättigung des Eisenkreises ein signifikanter Faktor für die Verursachung einer Längsfeldkomponente ist. Bereits in real auftretenden magnetischen Sättigungsbereichen 
kann das resultierende Entlastungsfeld höhere Werte annehmen als das durch die Sättigung verminderte Querfeld. In welcher Form der resultierende $k_{r}$-Faktor von der Sättigung beeinflusst wird, hängt dabei von der sättigenden Eisenstrecke und der Leiterquerschnittsgeometrie ab. Sind die Zähne von Sättigung betroffen, führt dies zur Ausbildung einer Längsfeldkomponente. Die verwendeten Leitergeometrien sind in der Realität sehr unterschiedlich. Bei Formspulen wird man aufgrund des gegenwärtigen Stands der Technik darauf bedacht sein, keine zu großen Leiterhöhen zu wählen. Dies kann mit Hinblick auf einen hohen Nutenfüllfaktor durchaus zu den betrachteten flachen, breiten Leitern führen. Bei diesen ist die Zunahme des $k_{\text {- }}$-Werts besonders gravierend, da sie anfällig für Längsfelder sind.

\subsubsection{E-Maschine mit gegossenen Spulen}

Die Auswirkung der in Abschnitt 5.1 beschriebenen Effekte der magnetischen Sättigung auf die Stromverdrängung wurden in Abschnitt 5.2.2 an einer vereinfachten Grundgeometrie mittels Finite-Elemente-Rechnungen nachgewiesen. In diesem Abschnitt sollen die Auswirkungen an einer real ausgelegten E-Maschine betrachtet werden. Dazu wird das Finite-Elemente-Modell der aus Kapitel 3 bekannten elektrischen Maschine mit gegossenen Spulen verwendet. In Abschnitt 5.2.2 konnten nur vereinfachende Annahmen bezüglich der sättigenden Bereiche getroffen werden. Daher ist es bei der realen E-Maschine zunächst interessant zu betrachten, welche Bereiche tatsächlich sättigen. Dies ist aus Abbildung 5-14 ersichtlich.

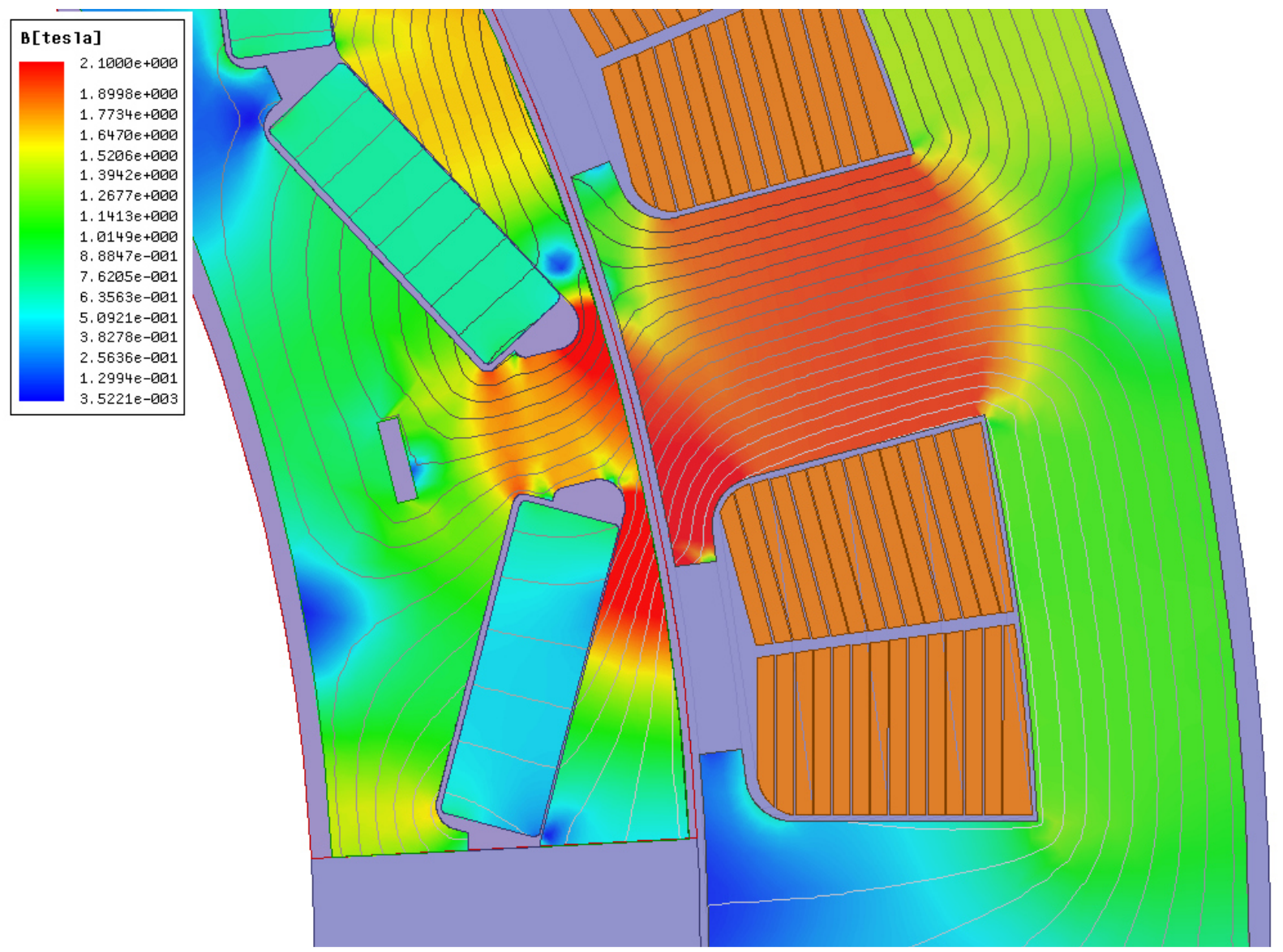

Abbildung 5-14: Magnetische Flussdichte und magnetischer Feldverlauf in Maschine mit Gussspulen bei maximaler Bestromung der betrachteten Spule 
In der Darstellung ist die magnetische Flussdichte im Eisenkreis bei maximalem Strom in der betrachteten Spule zu sehen. Bei dem gewählten Arbeitspunkt handelt es sich um den Eckpunkt - also ein Betriebspunkt mit hohem Strom von 419 A und gleichzeitig hoher Frequenz des Stroms. Die Stromfrequenz liegt bei rund $460 \mathrm{~Hz}$. Die Farbskala ist so gewählt, dass das verwendete Elektroblech oberhalb von "Hellgrün" in den Sättigungsbereich gelangt. Es ist gut zu erkennen, dass der Zahn, um den die maximal bestromte Spule gewickelt ist, stark gesättigt ist, ebenso wie Teile des Rotors. Das bedeutet, dass der vorliegende Fall nach Tabelle 4 zwischen den Varianten in den Spalten 1 und 3 liegt. In der Folge ist mit signifikanten Stromverdrängungsverlusten durch die magnetische Sättigung des Eisenkreises zu rechnen.

In Abbildung 5-15 sind die Auswirkungen der magnetischen Sättigung auf die einzelnen Leiterlagen der elektrischen Maschine gezeigt.
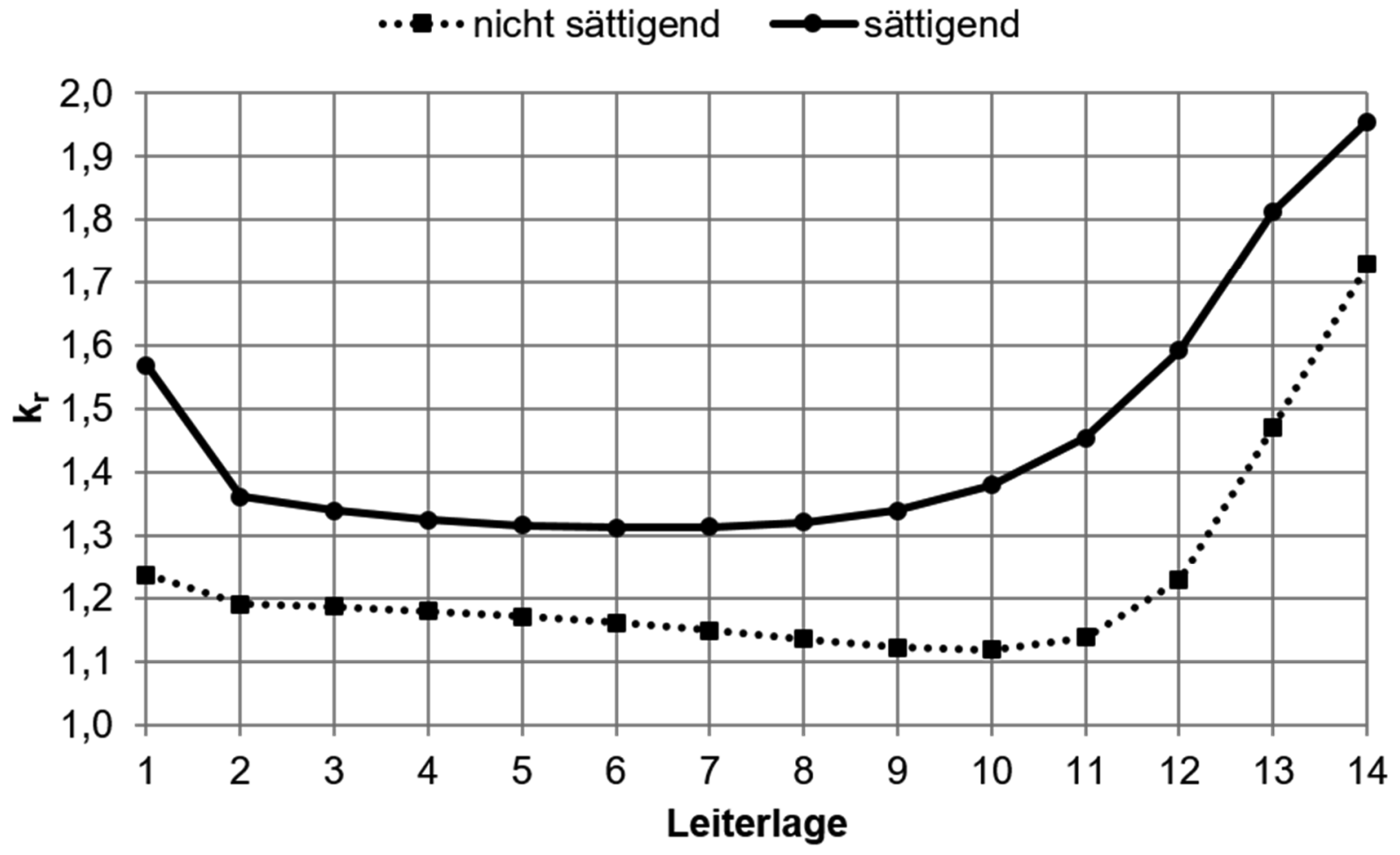

Abbildung 5-15: Gegenüberstellung des über FEM ermittelten mittleren Widerstandserhöhungsfaktors einer elektrischen Maschine mit gegossenen Spulen im Eckpunkt für eine Variante mit handelsüblichem sättigendem Elektroblech und eine Variante mit fiktivem nicht sättigenden Elektroblech gleicher Anfangspermeabilität

Die durchgezogene Linie stellt die Berechnungsergebnisse der regulären Variante mit sättigendem Blech dar. Als Vergleich wurde eine zweite Variante gerechnet, bei der ein fiktives, nicht sättigendes Blech angenommen wurde. Es wird deutlich, dass sich die Stromverdrängungsverluste bei der Verwendung des nicht sättigenden Blechs signifikant reduzieren. Der mittlere Widerstandserhöhungsfaktor sinkt von 1,46 auf 1,23. Ein interessantes Detail der Darstellung ist, dass sich die beiden Stromverdrängungsverläufe nicht schneiden, wie es bei den Verläufen in Abbildung 5-8 der Fall ist. Dies hängt wahrscheinlich mit dem in die Nuten eindringenden Permanentmagnetfeld beziehungsweise den sättigendenden Zahnköpfen zusammen. Durch die Sättigung bildet sich auch an den Zahnköpfen ein Entlastungsfeld aus, wie es in Abbildung 5-16 
an beiden Seiten zu sehen ist. Aus Abbildung 5-14 ist ersichtlich, dass die Zahnköpfe unterschiedlich stark in die Sättigung getrieben werden.

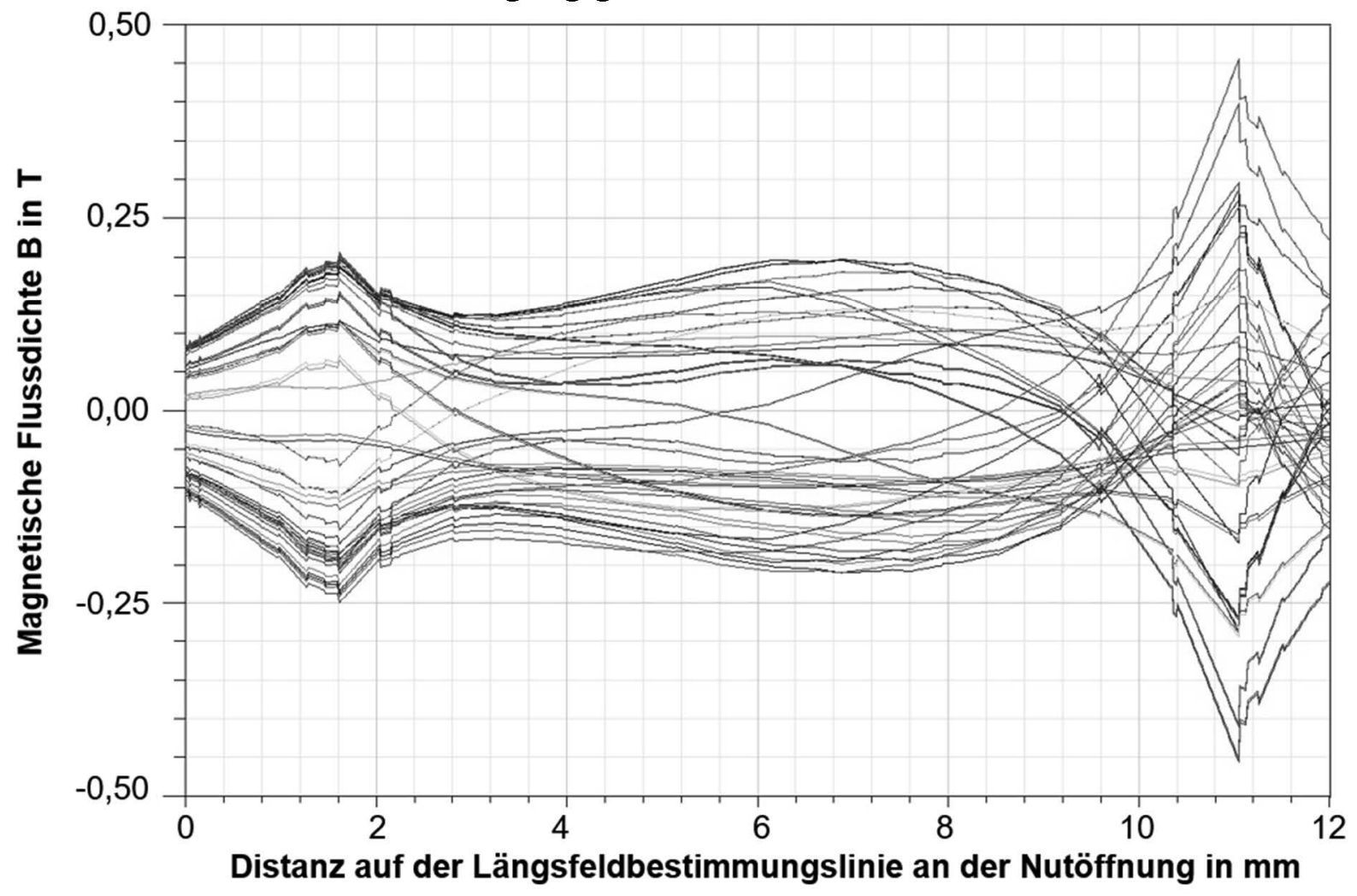

Abbildung 5-16: Magnetische Flussdichte in Längsrichtung an der Nutöffnung bei magnetischer Sättigung der Zahnköpfe

Die in der Literatur angeratenen Zahnköpfe zur Reduzierung der Stromverdrängung [36] [46] durch das in die Nut eindringende Hauptfeld sind also nur so lange hilfreich, wie sie nicht in Sättigung gelangen. Aus Auslegungssicht wird man versucht sein, die Zahnköpfe dünn zu gestalten, um möglichst wenig Nutraum dafür aufwenden zu müssen. Hierbei muss man sich im Klaren sein, dass die Stromverdrängung infolgedessen nur bei niedrigen Strömen, also bei geringen Drehmomentanforderungen, reduziert wird. Erwähnenswert ist noch, dass sich das Entlastungsfeld der Zahnköpfe asymmetrisch ausformt. Dies passt zu Gröningers Beobachtungen [16] ungleichmäßiger Verluste in den beiden Spulenhälften einer Nut in Abhängigkeit von motorischem oder generatorischem Betrieb.

Eine weitere Feststellung aus Abbildung 5-15 ist der starke Anstieg des $k_{r}$-Werts in der untersten Leiterlage bei sättigendem Eisen. Er ist auf einen stärkeren Anstieg des Längsfelds am Nutgrund zurückzuführen, wie er in Abbildung 5-6 zu erkennen ist. Der Längsfeldanstieg lässt sich wiederum durch die gesättigten Zähne und das nicht gesättigte Joch erklären. Dadurch kommt es in diesem Übergangsbereich zum Entlastungsfeld. Dies führt im unteren Nutbereich zu einer höheren magnetischen Flussdichte.

Zusammenfassend ist festzuhalten, dass sich die Auswirkungen der magnetischen Sättigung des Eisenkreises auf die Stromverdrängung auch in der Simulation eines vollständigen E-Maschinenmodells wiederfinden. 


\subsection{Ableitung analytischer Nährung}

Nachdem aufgezeigt wurde, wie sich die magnetische Sättigung des Eisenkreises auf das Nutfeld und die resultierende Stromverdrängung auswirkt, wird in diesem Abschnitt eine Weiterentwicklung des analytischen Ansatzes aus Abschnitt 2.1.4 vorgestellt. Diese ermöglicht es, die aus der magnetischen Sättigung entstehenden Verhältnisse analytisch zu bestimmen.

\subsubsection{Verminderung der Querfeldkomponente}

Die in Abschnitt 5.1.1 beschriebene Verminderung der Querfeldkomponente lässt sich recht einfach analytisch berücksichtigen. Für die Stromverdrängung erfolgt die Berücksichtigung analog zum Leiterbreite-zu-Nutbreite-Verhältnis, das in (2-22) enthalten ist. Das Vorgehen wird bereits bei Dabrowski u.a. [6] vorgeschlagen. Jedoch wird bei Dabrowski nicht weiter erläutert, wie sich der Faktor bestimmen lässt, mit dem die Verminderung berücksichtigt werden soll. Dies wird im Folgenden weiterführend untersucht.

In Abschnitt 4.3 wurde gezeigt, dass der $k_{r}$-Wert konstant bleibt, sofern die Streufeldanteile linear mit dem Strom ansteigen. Die Verhältnisse im Sättigungsfall werden mit Hilfe der Abbildung 5-17 betrachtet.

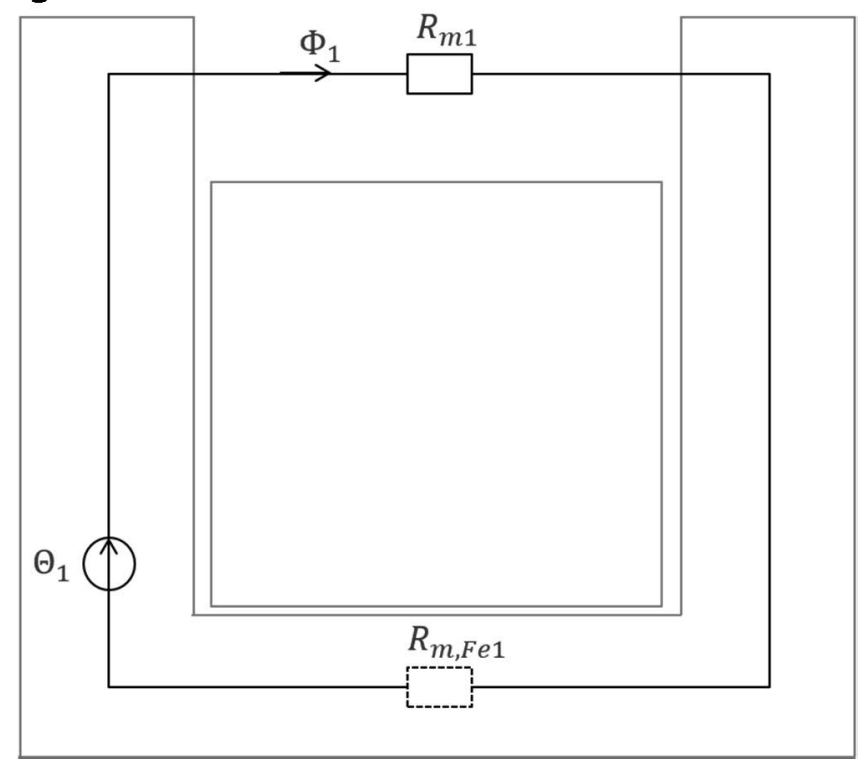

Abbildung 5-17: Vereinfachtes Ersatzschaltbild des magnetischen Kreises zur Herleitung des Verminderungsfaktors des Nutquerfelds bei magnetischer Sättigung

Das Nutquerfeld stellt in dieser vereinfachten Darstellung eine Reihenschaltung von zwei magnetischen Widerständen dar. Für diese gilt

$$
\Theta_{1}=\phi_{1}\left(R_{m 1}+R_{m, F e 1}\right)
$$

Zuvor galt

$$
\Theta_{m 1}=\phi_{1} R_{m 1}
$$


da $R_{m, F e 1}=0$ angenommen wurde. Für einen gesättigten Eisenkreis ist dies nicht mehr der Fall. Für diesen muss der verringerte magnetische Spannungsabfall in der Nut bei der Bestimmung der Stromverdrängung berücksichtigt werden. Dies geschieht über das Verhältnis

$$
a_{q f}\left(B_{z}\right)=\frac{R_{m 1}}{R_{m 1}+R\left(B_{z}\right)_{m, F e 1}} .
$$

Es gibt an, in welchem Maße das Nutquerfeld und damit auch die Stromverdrängung vermindert werden. Das Verhältnis wird als Verminderungsfaktor $a_{q f}$ bezeichnet und ist vom Sättigungszustand des Eisenkreises abhängig, da die relative Permeabilität des Eisenkreises $\mu_{r, F e}$ keine Konstante ist. Es ist exemplarisch für die vorliegende Geometrie und das verwendete NO30-Elektroblech von C.D. Wälzholz in Abbildung 5-18 dargestellt.

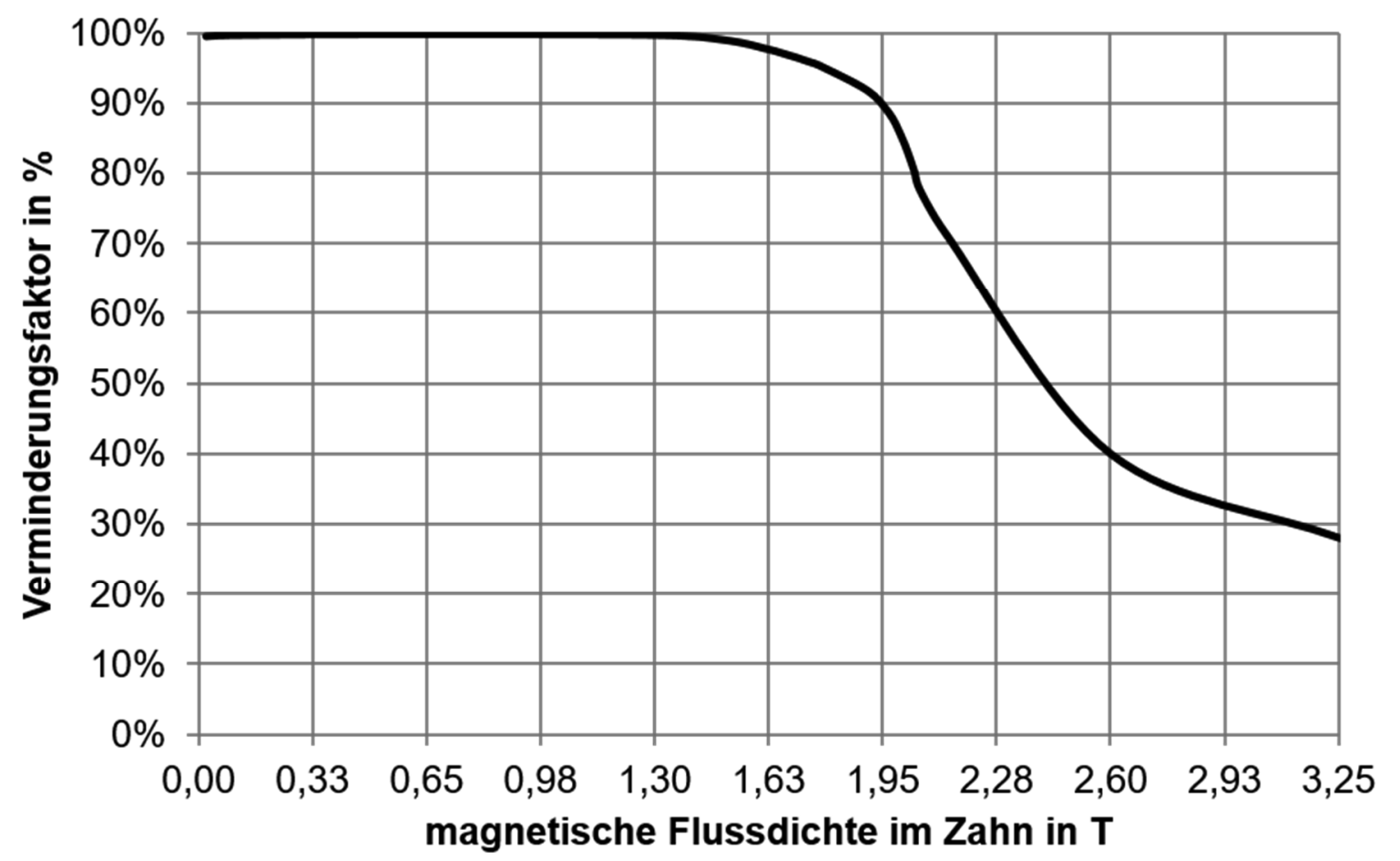

Abbildung 5-18: Durch die magnetische Sättigung verursachter Verminderungsfaktor $a_{q f}$ für das Querfeld

Die Verhältnisse bei der Verminderung des Querfelds durch die Sättigung des Eisenkreises sind vergleichbar mit einem Leiter, der schmaler als die Nut ist, in der er liegt. Denn in beiden Fällen nimmt der magnetische Widerstand außerhalb des Kupfers zu. Der Verminderungsfaktor $a_{q f}$ wird vor dem Leiterbreite-zu-Nutbreite-Verhältnis in Formel (2-22) ergänzt:

$$
\alpha_{s q f}=\sqrt{\pi f \mu_{0} \kappa a_{q f}\left(B_{z}\right) \frac{b_{l}}{b_{n}}} .
$$


In Abbildung 5-19 sind die analytisch bestimmten, verminderten $k_{r}$-Verläufe für verschiedene Ströme, also verschiedene magnetische Sättigungszustände, exemplarisch dargestellt.

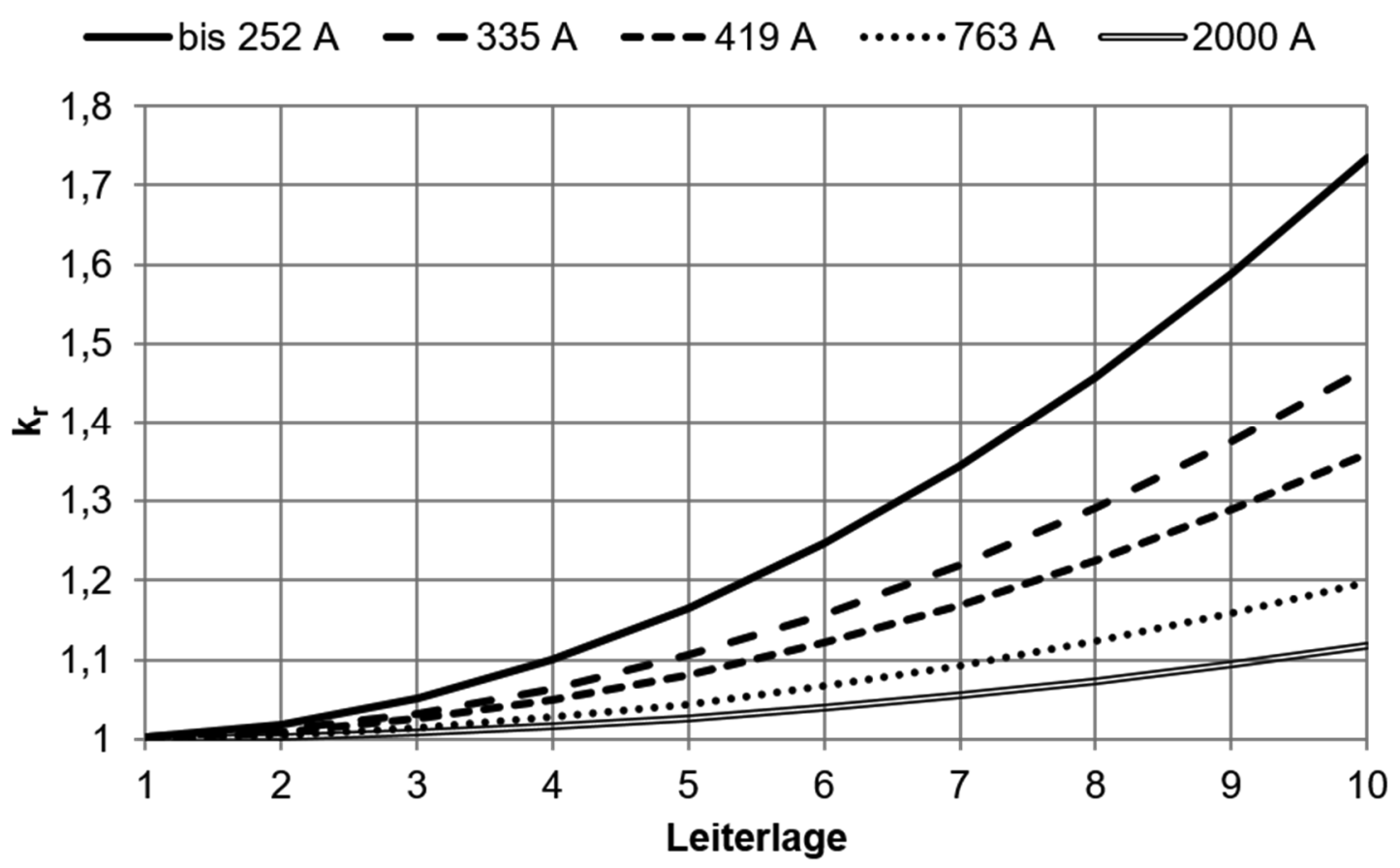

Abbildung 5-19: Analytisch ermittelte Verminderung des $k_{r}$-Verlaufs durch magnetische Sättigung, aufgetragen für verschiedene Ströme und Sättigungszustände (bis 1,77 T; 1,91 T; $2 \mathrm{~T} ; 2,2 \mathrm{~T} ; 2,67 \mathrm{~T}$ )

Auch hier ist gut zu erkennen, wie stark sich die Verminderung der Querfeldkomponente beim Eintritt in den Sättigungsbereich auswirkt. Dies deckt sich mit den Beobachtungen aus Abschnitt 5.2.

Die Hochkantleiter aus Abschnitt 5.2.2.4 bieten sich aufgrund ihrer geringen Anfälligkeit für Längsfeldeinflüsse und ihrer hohen Sensibilität für Querfelder an, um zu überprüfen, ob sich mit Hilfe des in diesem Abschnitt vorgeschlagenen Vorgehens die Verminderung der Querfeldkomponente auf analytischem Wege ausreichend genau berücksichtigen lässt. Dazu wird die in Abbildung 5-11 gezeigte Hochkantleiteranordnung für verschiedene Sättigungen der Zähne betrachtet. In Abbildung 5-20 sind die mittleren $k_{r}$-Verläufe für verschiedene Ströme und damit magnetische Sättigungen dargestellt. 
- analytisches Verfahren nach Abschnitt 2.1.4

- Finite-Elemente-Berechnung

•.. angepasstes analytisches Verfahren nach Abschnitt 5.3.1

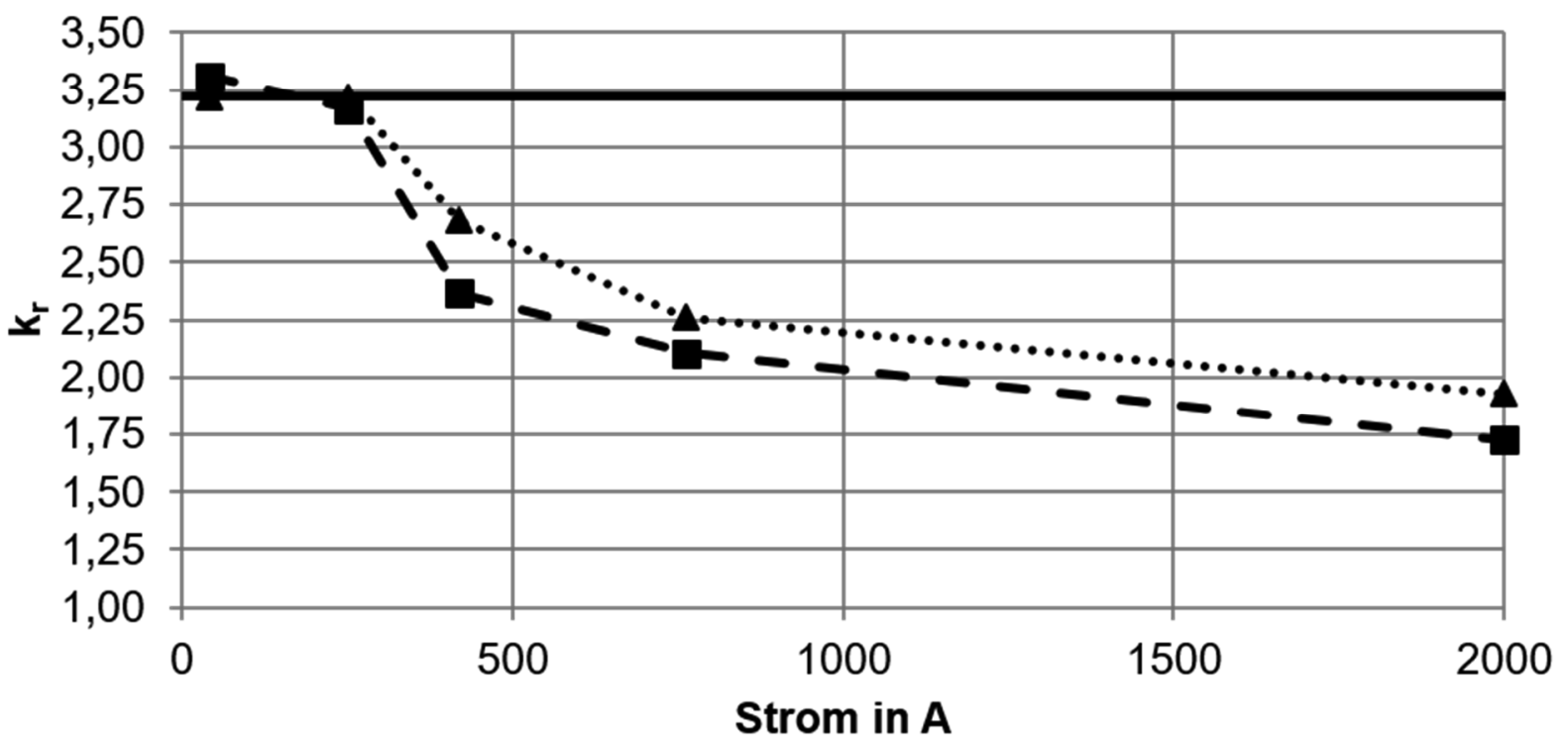

Abbildung 5-20: Gegenüberstellung von mittleren $k_{r}$-Verläufen, die mittels analytischer Verfahren unter Berücksichtigung des Querfelds und mittels Finite-Elemente-Berechnungen für verschiedene Ströme und damit verschiedene magnetische Sättigungszustände des Eisenkreises bei einer Frequenz von $933 \mathrm{~Hz}$ bestimmt wurden

Dabei werden drei Berechnungsansätze einander gegenübergestellt. Der aus Abschnitt 2.1.4 bekannte analytische Ansatz vernachlässigt den magnetischen Sättigungszustand des Eisenkreises bei der Bestimmung des Widerstandserhöhungsfaktors $k_{r}$. Daher ergibt dieser Ansatz für alle Ströme einen konstanten Widerstandserhöhungsfaktor. Die Berechnung mittels Finite-Elemente-Ansatz zeigt, dass der Widerstandserhöhungsfaktor mit dem Eintritt in den magnetischen Sättigungsbereich signifikant abnimmt. Dies ist auf die Verminderung der Querfeldkomponente zurückzuführen. Der in diesem Abschnitt beschriebene analytische Ansatz ist in der Lage, den $k_{\digamma}$-Verlauf in groben Zügen nachzubilden, wie ebenfalls aus Abbildung 5-20 hervorgeht.

\subsubsection{Zahnentlastungsfeld - Entstehen einer Längsfeldkomponente}

Längsfeldanteile werden in der Literatur in Bezug auf Stromverdrängung noch nicht analytisch behandelt. Eine Ausnahme stellt das Eindringen des Luftspaltfelds in die Nut dar [6] [38] [50]. Doch mit dem Nutquerfeld und dem in die Nuten eindringenden Hauptfeld lassen sich $k_{r}$-Verläufe, die im unteren Nutbereich wieder ansteigen, nicht plausibel erklären, wie sie beispielsweise in Abbildung 5-15 zu sehen sind. Bei diesem Beispiel liegt der Widerstandserhöhungsfaktor trotz flacher Leiter bereits in der untersten Leiterlage deutlich über 1. Es ist unwahrscheinlich, dass das Hauptfeld so tief in die Nut eindringt. Durch einen Querfeldanteil kann die Stromverdrängung in den untersten Lagen aufgrund der flachen Leiter nicht entstehen. 
Wie in Abschnitt 5.2 gezeigt wurde, können Entlastungsfelder die Ursache der zusätzlichen Stromverdrängung sein. Für die Stromverdrängung durch Entlastungsfelder in Längsrichtung, wie sie durch die Entlastung der Zähne entstehen, findet sich in der Literatur noch keine Möglichkeit zur analytischen Berücksichtigung. Bei dem im Folgenden vorgestellten analytischen Ansatz handelt es sich daher um eine Eigenentwicklung, die auf den durchgeführten Untersuchungen basiert. Für diesen Ansatz ist eine neuartige Modellvorstellung von Nöten. Diese besagt, dass neben dem reinen Nutquerfeld auch ein reines Nutlängsfeld existiert, wie es als Ersatzschaltbild in Abbildung 5-21 zu sehen ist. Der resultierende Widerstandserhöhungsfaktor ergibt sich zusammen aus Querfeld- und Längsfeldkomponente.

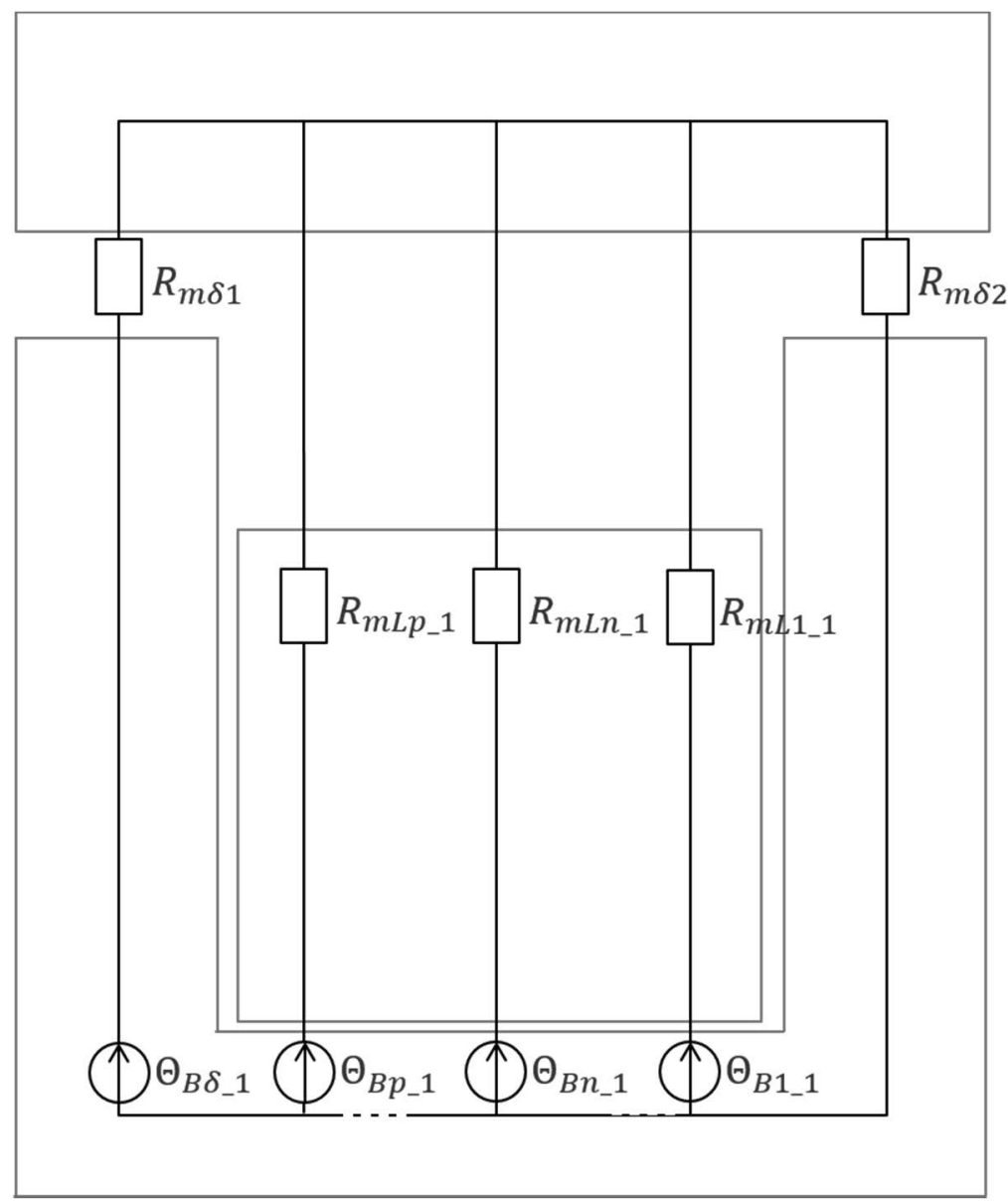

Abbildung 5-21: Ersatzschaltbild der Längsfeldkomponente des Nutfelds

Die prinzipielle Beschaffenheit des Zahnentlastungsfelds kann der Abbildung 5-2 entnommen werden, die den Verlauf des Längsfelds auf Basis einer Finite-ElementeBerechnung zeigt. Das Zahnentlastungsfeld entspricht in seiner Form entfernt dem Nutquerfeld. Der Nutquerfeldverlauf ist in Abbildung 4-4 zu sehen. Eine Ähnlichkeit besteht, wenn man den Verlauf des Zahnentlastungsfeldes in Abbildung 5-2 von der Nutmitte hin zu den Zahnseiten betrachtet. Der Anstieg ähnelt dann dem Anstieg des Querfelds vom Nutgrund zur Nutöffnung hin. Basierend auf dieser Ähnlichkeit wird die Annahme getroffen, dass die Gesetzmäßigkeiten des Nutquerfelds auch für die durch Zahnentlastung entstehende Längsfeldkomponente gelten. Der Reduktionsfaktor in Längsrichtung $a_{s / f}$ berechnet sich gemäß 


$$
\alpha_{s l f}=\sqrt{\pi f \mu_{0} \kappa a_{l \mathrm{f}}(I) a_{r}} .
$$

Dabei berücksichtigt der Faktor $a_{r}$ die geringere Intensität des Längsfelds aufgrund eines höheren magnetischen Widerstands außerhalb des elektrischen Leiters. Der Faktor $a_{\text {If }}$ wird analog zum Verminderungsfaktor $a_{q f}$ ermittelt. Dabei stellt das Entlastungsfeld eine Parallelschaltung von magnetischen Widerständen dar, wie aus Abbildung 5-22 hervorgeht.

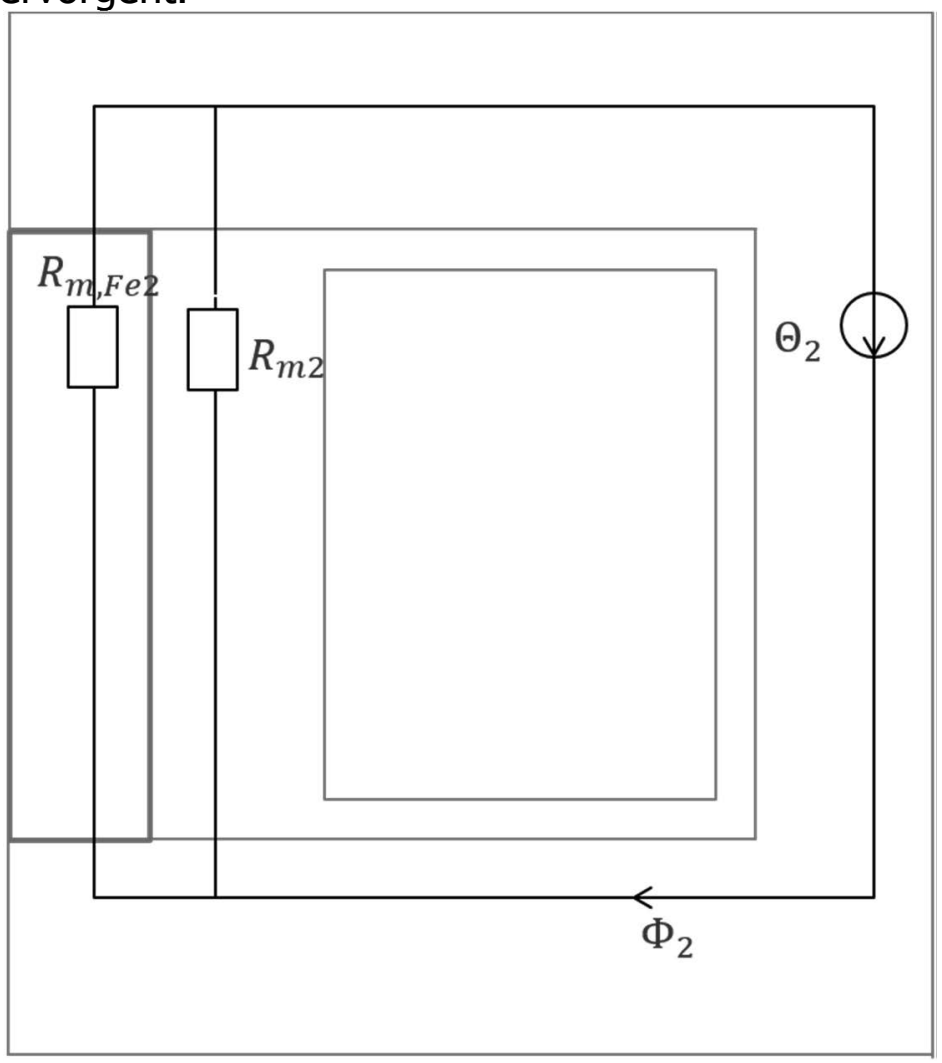

Abbildung 5-22: Vereinfachtes Ersatzschaltbild des magnetischen Kreises zur Herleitung des Verminderungsfaktors des Nutlängsfelds bei magnetischer Sättigung

$R_{m, F e 2}$ stellt einen gesättigten Abschnitt des Eisenkreises dar, für den nicht $R_{m, F e 2}=0$ angenommen werden kann. Daneben bildet sich im Nutraum der Entlastungspfad $R_{m 2}$ aus. Die beiden magnetischen Widerstände sind parallel geschaltet. Auch hier ergibt sich ein magnetisches Widerstandsverhältnis, das angibt in welchem Maße ein Nutlängsfeld entsteht.

$$
a_{l f}\left(B_{z}\right)=\frac{R\left(B_{z}\right)_{m, F e 2}}{R_{m 2}+R\left(B_{z}\right)_{m, F e 2}} .
$$

Das Verhältnis ist exemplarisch für die vorliegende Geometrie und das verwendete NO30-Elektroblech von C.D. Wälzholz in Abbildung 5-23 dargestellt. 


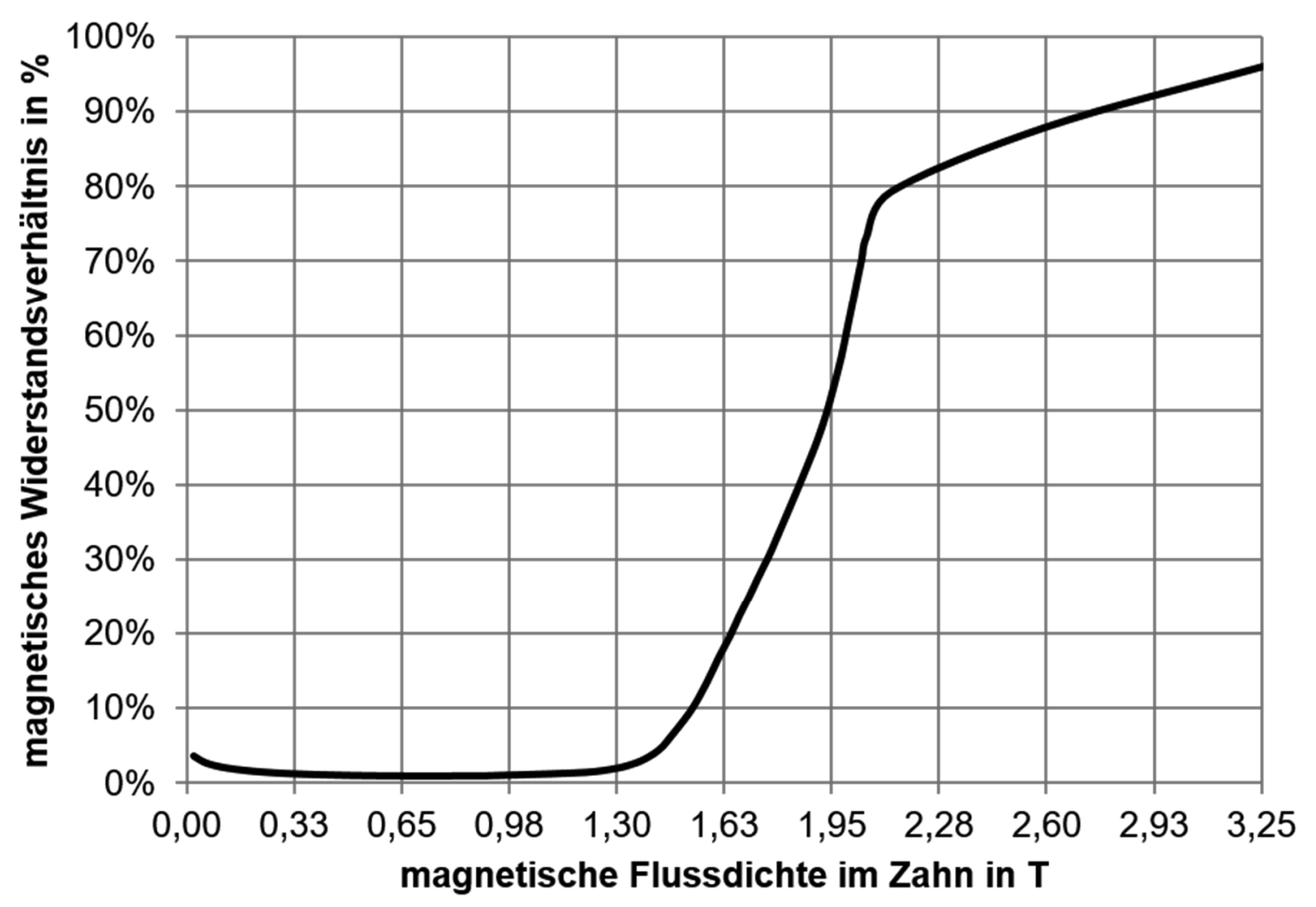

Abbildung 5-23: Durch die magnetische Sättigung verursachter Faktor für das Längsfeld

Die magnetische Flussdichte in Längsrichtung erreicht erst mit dem Eintritt in die magnetische Sättigung signifikante Werte. Nach der Bestimmung des Reduktionsfaktors $a_{\text {If }}$ berechnet sich $\beta_{\text {lf }}$ gemäß

$$
\beta_{l f}=\alpha_{l f} b_{l} .
$$

Anders als in Gleichung (2-21) geht hier die Leiterbreite als kritische geometrische Größe ein.

Der Widerstandserhöhungsfaktor durch die Längskomponente $k_{\text {rslf }}$ lasst sich mit Hilfe der Gleichungen (2-18), (2-19) und (2-20) bestimmen. Der resultierende Gesamtwiderstandserhöhungsfaktor ergibt sich nach

$$
k_{r s}=k_{r s q f}+k_{r s l f} .
$$

Dabei entspricht $k_{r s q f}$ dem Stromverdrängungsanteil, der auf das Querfeld zurückzuführen ist. Die Gleichung leitet sich aus Richters Untersuchungen [50] her. Richter betrachtet die Stromverdrängung, die sich aus dem resultierenden Feld ergibt. Da sich hier zwei Wirbelströmungen überlagern, die jeweils symmetrisch zu den beiden senkrecht aufeinander stehenden Mittelebenen des Leiters sind, kann die Phasenverschiebung $\zeta$ zwischen den beiden Wirbelströmungen vernachlässigt werden. 
Abbildung 5-24 zeigt den resultierenden Gesamtwiderstandserhöhungsfaktor für verschiedene Sättigungszustände des magnetischen Kreises. Diese werden wieder über den Strangstrom eingestellt.

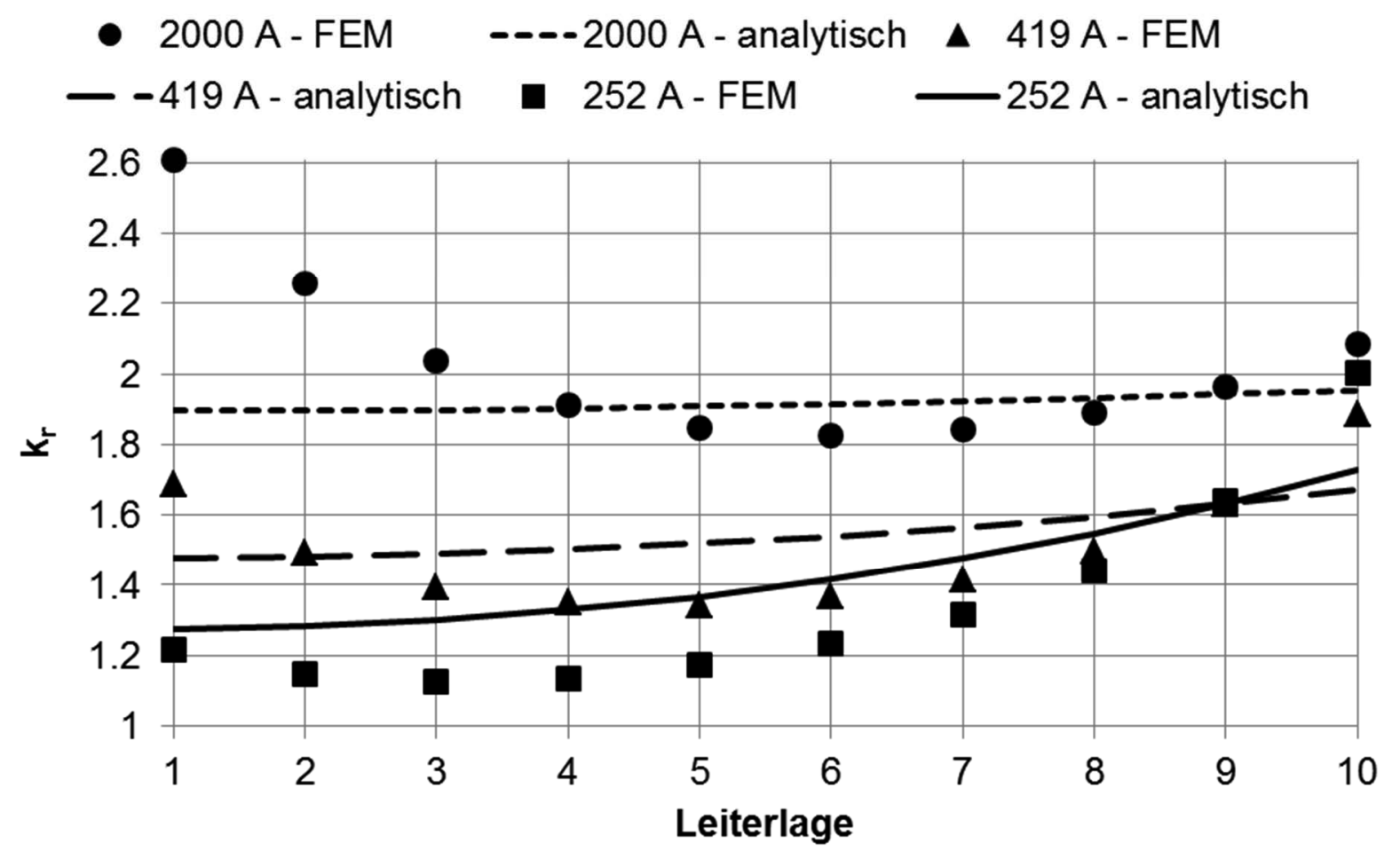

Abbildung 5-24: Gegenüberstellung des neu entwickelten analytischen Verfahrens und einer Finite-Elemente-Berechnung anhand der $k_{r}$-Verläufe für verschiedene magnetische Sättigungsgrade $(1,77 \mathrm{~T} ; 2 \mathrm{~T} ; 2,67 \mathrm{~T})$

Dabei sind die Ergebnisse der Finite-Elemente-Rechnungen als Symbole aufgetragen. Diesen sind die Ergebnisse der mit dem eben beschriebenen analytischen Verfahren durchgeführten Berechnungen als Linien gegenübergestellt. Es sind deutliche Abweichungen zwischen beiden Berechnungswegen zu erkennen. Es besteht jedoch Übereinstimmung im grundsätzlichen Anstiegsverhalten. So ist das entwickelte analytische Verfahren in der Lage, den Niveauanstieg des $k_{r}$-Verlaufs durch zunehmende magnetische Sättigung im Eisenkreis nachzubilden. Das herkömmliche analytische Verfahren kann dies nicht leisten. Es bietet insbesondere keine plausible Erklärung für den Anstieg des $k_{r}$-Verlaufs in den unteren Leiterlagen.

Eine Ursache für die Abweichungen des angepassten analytischen Verfahrens gegenüber der Finite-Elemente-Berechnung dürfte die vereinfachende Annahme eines Längsfelds mit konstanter Intensität über die gesamte Nuthöhe sein. Hier könnte bei höherer Detailierung des analytischen Verfahrens sicherlich eine bessere Annäherung an die Finite-Elemente-Berechnungen erreicht werden. Doch die exakte Nachbildung der Ergebnisse ist zunächst nicht das Ziel. Vielmehr soll eine neue Modellvorstellung geschaffen werden, nach der die Stromverdrängung sowohl von einer Querfeld- als auch von einer Längsfeldkomponente abhängt. Die Sättigung des magnetischen Kreises hat dabei Einfluss, wie hoch die Querfeld- und die Längsfeldkomponente des Nutfelds sind. Mit zunehmender Sättigung der Zähne beeinflusst das Längsfeld mehr und mehr die Stromverdrängung, während der Einfluss des Querfelds schwindet. Für die 
Bestätigung dieses grundsätzlichen Mechanismus scheinen die Berechnungen auszureichen.

Dies lässt sich auch anhand des mittleren $k_{r}$-Faktors erkennen, der in Abbildung 5-25 dargestellt ist.

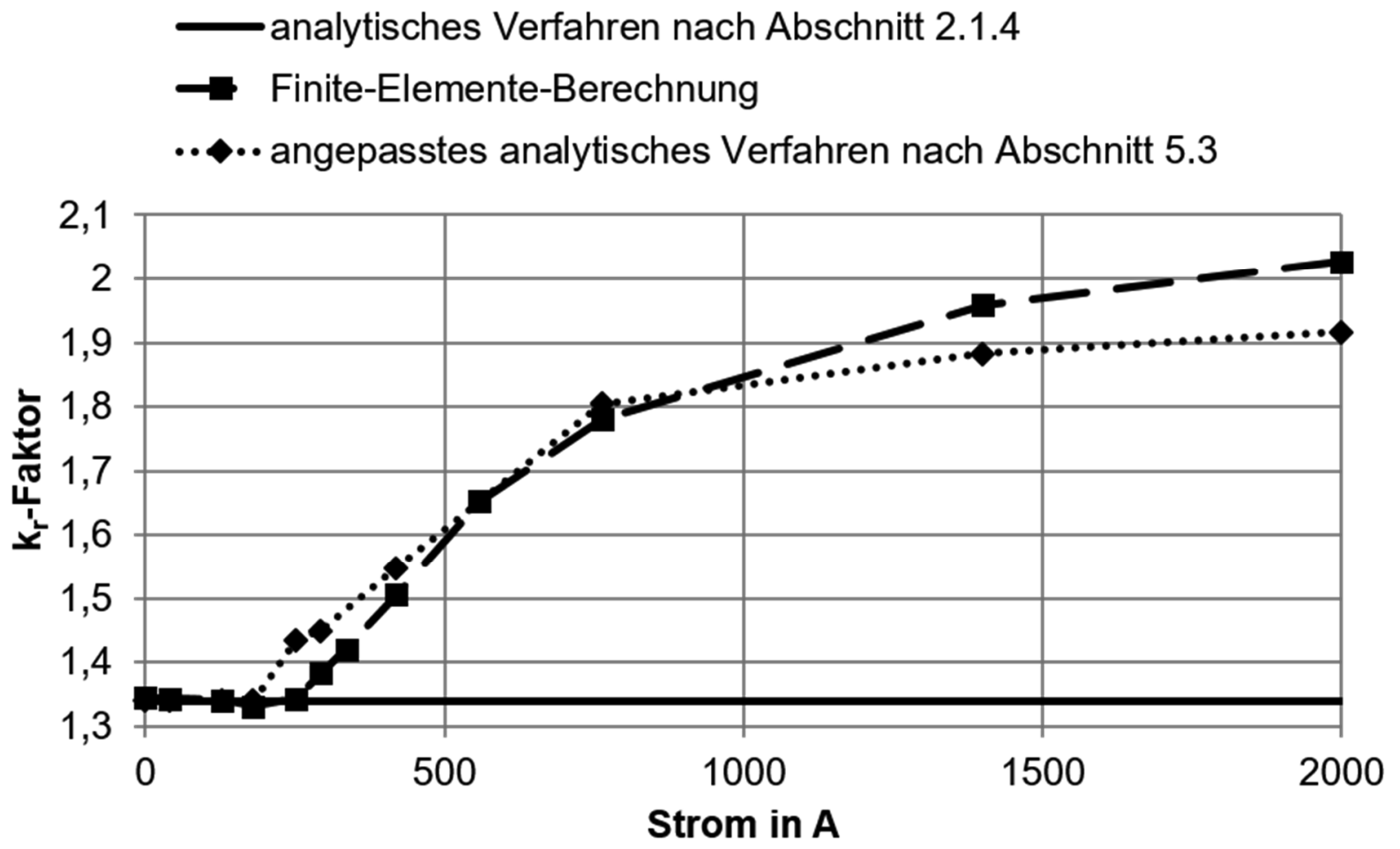

Abbildung 5-25: Gegenüberstellung von mittleren $k_{\Gamma}$-Verläufen, die mittels analytischer Verfahren und Finite-Elemente-Berechnungen für verschiedene Ströme und damit verschiedene magnetische Sättigungszustände des Eisenkreises bei einer Frequenz von $933 \mathrm{~Hz}$ bestimmt wurden

Das bekannte analytische Verfahren, das in Abschnitt 2.1.4 beschrieben wird, berücksichtigt die Auswirkungen der magnetischen Sättigung des Eisenkreises auf die Stromverdrängung nicht und ergibt daher einen über sämtliche Strangströme konstanten Widerstandserhöhungsfaktor. Dies deckt sich nicht mit den Ergebnissen der Finite-Elemente-Rechnung, die ab ungefähr $250 \mathrm{~A}$ einen deutlichen Anstieg des $k_{r}$ Faktors ergibt. Das sättigungsbedingte Verhalten lässt sich mit Hilfe des in diesem Abschnitt beschriebenen angepassten analytischen Verfahrens in guter Näherung bestimmen.

In Abbildung 5-26 sind die $k_{r}$-Verläufe für verschiedene Frequenzen bei einem konstanten Strom von 419 A aufgetragen, der zu einer konstanten Sättigung des Eisenkreises führt. Die Zahninduktion liegt dadurch bei $2 \mathrm{~T}$. 

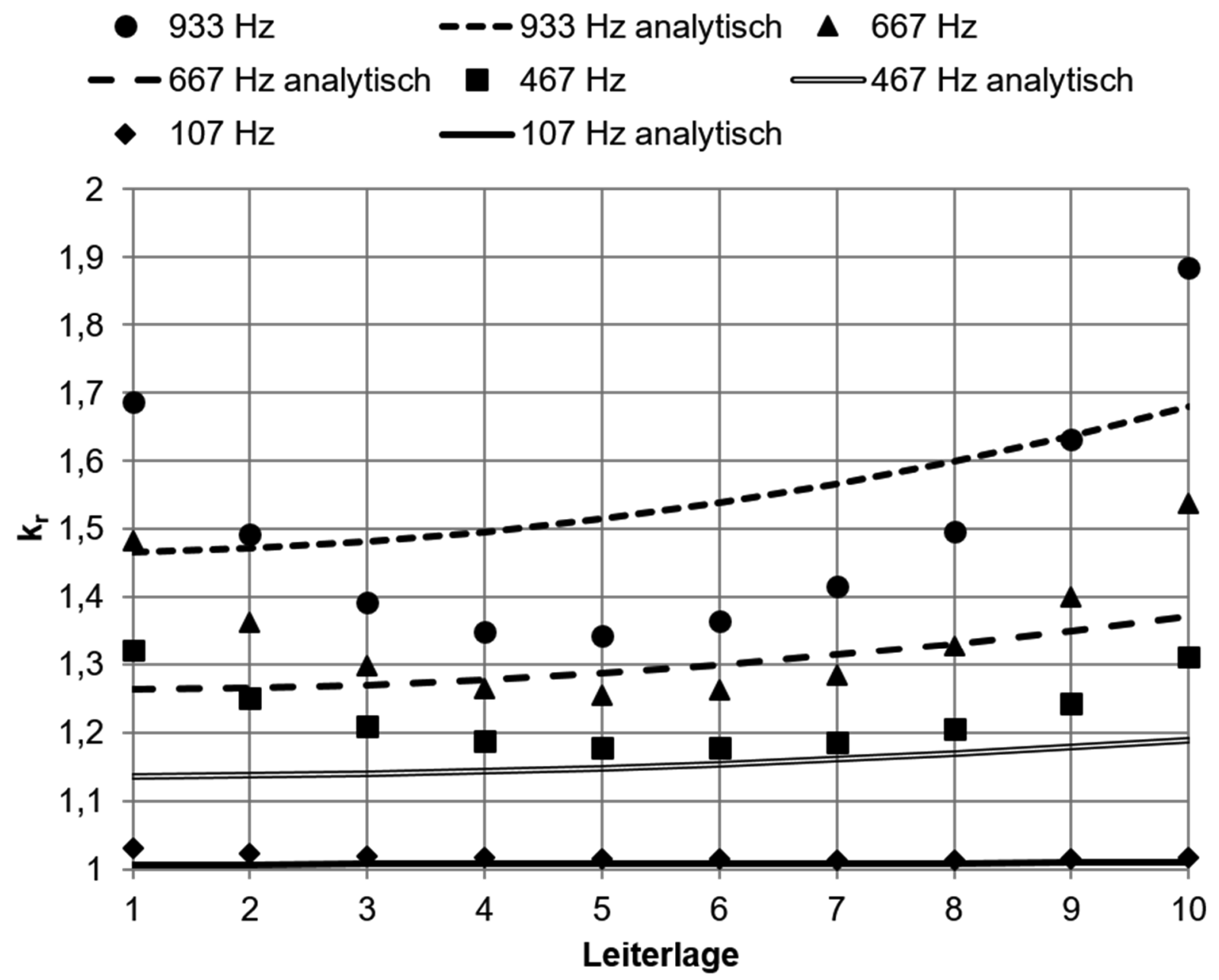

Abbildung 5-26: Gegenüberstellung des neu entwickelten analytischen Verfahrens und einer Finite-Elemente-Berechnung anhand der $k_{r}$-Verläufe für verschiedene Frequenzen

Wie in Abbildung 5-24 sind die Finite-Elemente-Berechnungen als Symbol für die einzelnen Leiterlagen dargestellt, während die Ergebnisse der analytischen Berechnungen als Linien aufgetragen sind. Auch hier ist zu erkennen, dass die analytische Berechnung in guter Näherung mit der Finite-Elemente-Rechnung übereinstimmt oder zumindest die gleichen Tendenzen aufweist. Währenddessen ist es mit der Annahme eines reinen Querfelds nicht mehr möglich, die entstehenden $k_{r}$ Verläufe vorherzusagen. Dies geht aus Abbildung 5-27 hervor.

Hier sind entsprechend der Abbildung 5-25 die mittleren $k_{r}$-Verläufe für den Sättigungszustand bei 419 A für verschiedene Frequenzen des Stroms aufgetragen. Es ist zu sehen, dass sich das angepasste analytische Verfahren in guter Näherung mit der Finite-Elemente-Berechnung deckt, während die Annahme eines reinen Querfelds nach dem analytischen Verfahren aus Abschnitt 2.1.4 zu deutlich geringeren $k_{r}$ Werten führt. 


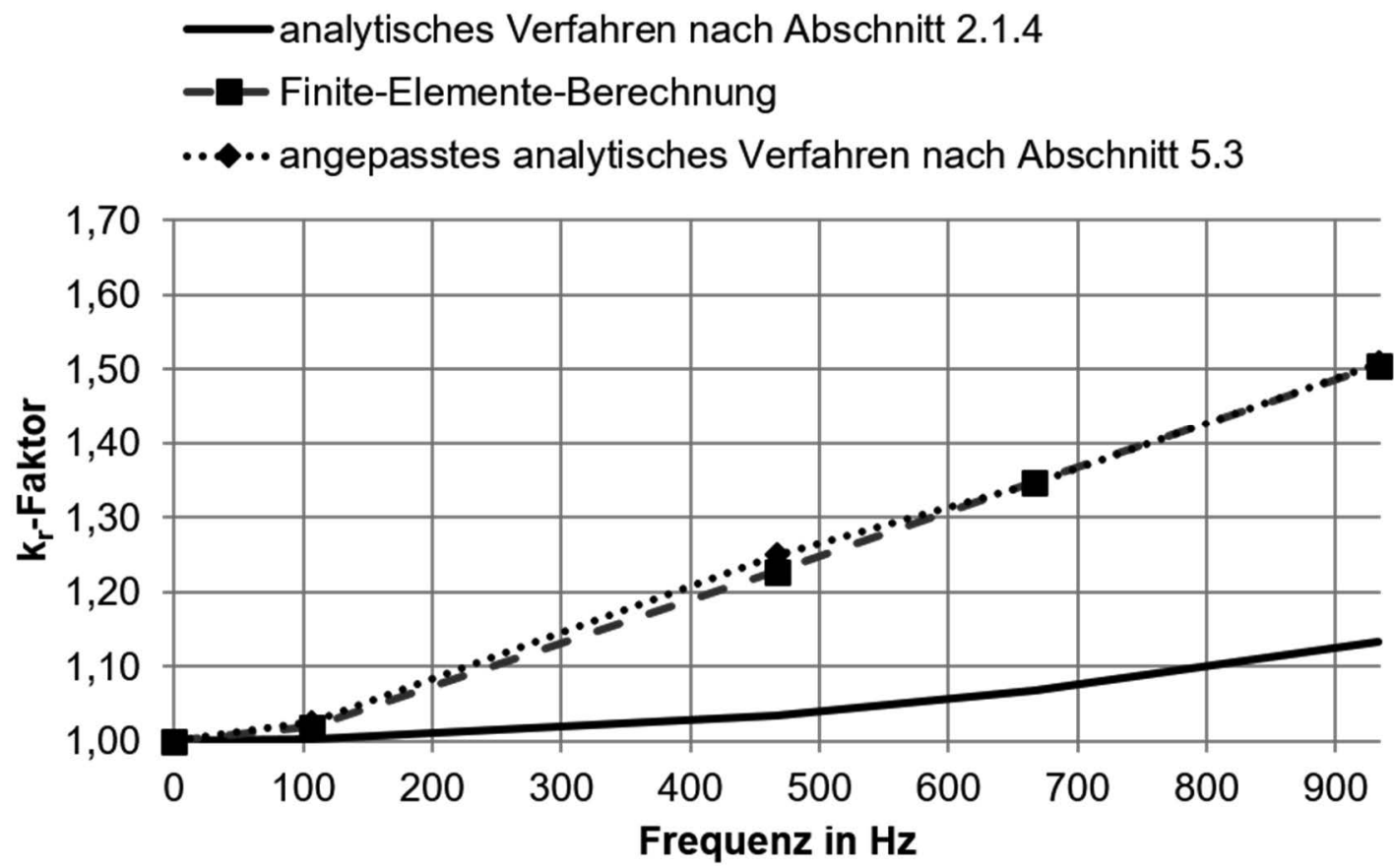

Abbildung 5-27: Gegenüberstellung von mittleren $k_{r}$-Verläufen, die mittels anayltischer Verfahren und Finite-Elemente-Berechnungen für verschiedene Frequenzen bei einem Strom von $419 \mathrm{~A}$ und damit bei einem konstanten magnetischen Sättigungszustand bei einer Zahninduktion von $2 \mathrm{~T}$ bestimmt wurden

\subsection{Zusammenfassung des Kapitels}

In diesem Kapitel werden die Auswirkungen der magnetischen Sättigung des Eisenkreises auf die Stromverdrängung behandelt. Es werden zwei wesentliche Beeinflussungen der Stromverdrängung ausgemacht: Die Verminderung des Querfelds wird durch die Zunahme des magnetischen Widerstands des Eisenkreises verursacht. Der Effekt wirkt sich reduzierend auf die Stromverdrängung aus. Zum anderen treten Entlastungsfelder entlang gesättigter Eisenstrecken auf und führen zu zusätzlicher Stromverdrängung. Hierbei wird insbesondere die Zahnsättigung weiterführend betrachtet. Bei magnetischer Sättigung der Zähne verläuft das Entlastungsfeld in Längsrichtung der Nut. Im Rahmen des Kapitels wird das Zusammenspiel zwischen Querfeld- und Längsfeldkomponente aufgezeigt. Am eindrucksvollsten lässt es sich am Vergleich zwischen einer Spule mit flach in der Nut liegenden Leitern und einer mit hochkant angeordneten Leitern erkennen. Dieser Fall wird in Abschnitt 5.2.2.2 betrachtet. Er zeigt, dass die Stromverdrängung bei Hochkantleitern mit der Sättigung der Zähne abnimmt, während sie bei flach in der Nut liegenden Leitern mit der Sättigung zunimmt.

Die Auswirkungen der magnetischen Sättigung des Eisenkreises werden anschlieBend an einer Beispielmaschine betrachtet. Hieraus können auch Erkenntnisse über die Art gewonnen werden, in der der magnetische Kreis sättigt. Abschließend wird aus den gemachten Beobachtungen ein analytisches Berechnungsverfahren abgeleitet. Dessen Ergebnisse decken sich vom prinzipiellen Verlauf und den Änderungstendenzen mit den Ergebnissen der vergleichenden Finite-ElementeRechnungen. 
5 Magnetische Sättigung 


\section{Phasenverschobene Spulenströme in einer Nut}

Das ursprüngliche Formelwerk von Sommerfeld [55], Field [11] und Emde [8], wie es in Abschnitt 2.1.4 vorgestellt wird, deckt den Fall von zwei zu verschiedenen Strängen gehörenden Spulenseiten in einer Nut nicht ab. Ein lange unbeachteter Sonderfall liegt vor, wenn die beiden Spulenseiten nebeneinander in der Nut liegen. Eine überwiegend analytische Betrachtung dieser Anordnung findet sich bei Mosebach [32]. Er leitet aus seinen Berechnungen einen Verminderungsfaktor für den $\mathrm{k}_{r}$ Wert ab. Mit diesem reduziert sich der resultierende $k_{-}$-Wert mit zunehmender Phasenverschiebung der beiden nebeneinanderliegenden Spulenseiten. $\mathrm{Zu}$ ähnlichen Ergebnissen kommt auch Gröninger u.a. [16] mit seinen Finite-ElementeBerechnungen. Allerdings findet sich bei Gröninger bereits ein davon abweichendes Ergebnis: Bei flachen, breiten Leitern steigt bei ihm der $k_{r}$-Wert mit zunehmender Phasenverschiebung leicht an. Interessant ist in diesem Zusammenhang auch die Arbeit von Endert u.a. [10]. Aus dieser ist ersichtlich, dass eine nebeneinanderliegende Anordnung von zwei zu verschiedenen Strängen gehörenden Spulenseiten in einer Nut zu stärker Stromverdrängung führt als eine übereinanderliegende Anordnung der Spulenseiten. Die Verminderung des Widerstandserhöhungsfaktors durch zwei zu verschiedenen Strängen gehörenden, nebeneinanderliegenden Spulenseiten in einer Nut lässt sich also nicht immer beobachten. Dieser Umstand soll im vorliegenden Kapitel näher untersucht werden.

\subsection{Vorüberlegungen}

Zunächst soll die zugrundeliegende Situation betrachtet werden, wie sie in Abbildung 6-1 dargestellt ist.

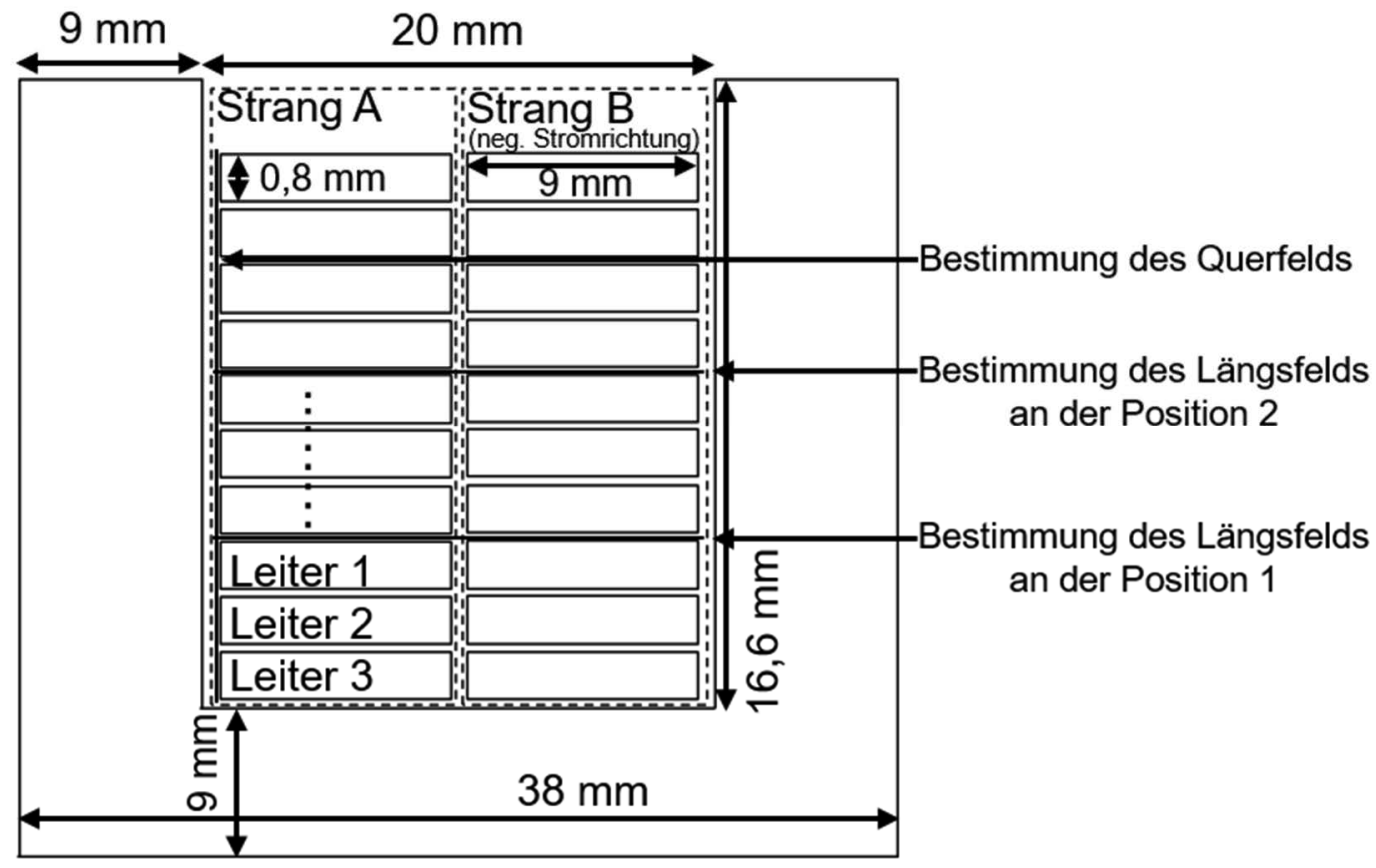

Abbildung 6-1: Schematische Leiteranordnung in der Nut bei Zahnspulen 
Die Spulenseiten der Stränge A und B sind nebeneinanderliegend in der Nut angeordnet. Werden sie phasen- und betragsgleich bestromt, ergibt sich kein signifikanter Unterschied zu der in Abbildung 4-3 betrachteten Leiteranordnung. Die Ströme in beiden Strängen über der Zeit bei phasenverschobener Bestromung sind in Abbildung 6-2 dargestellt.

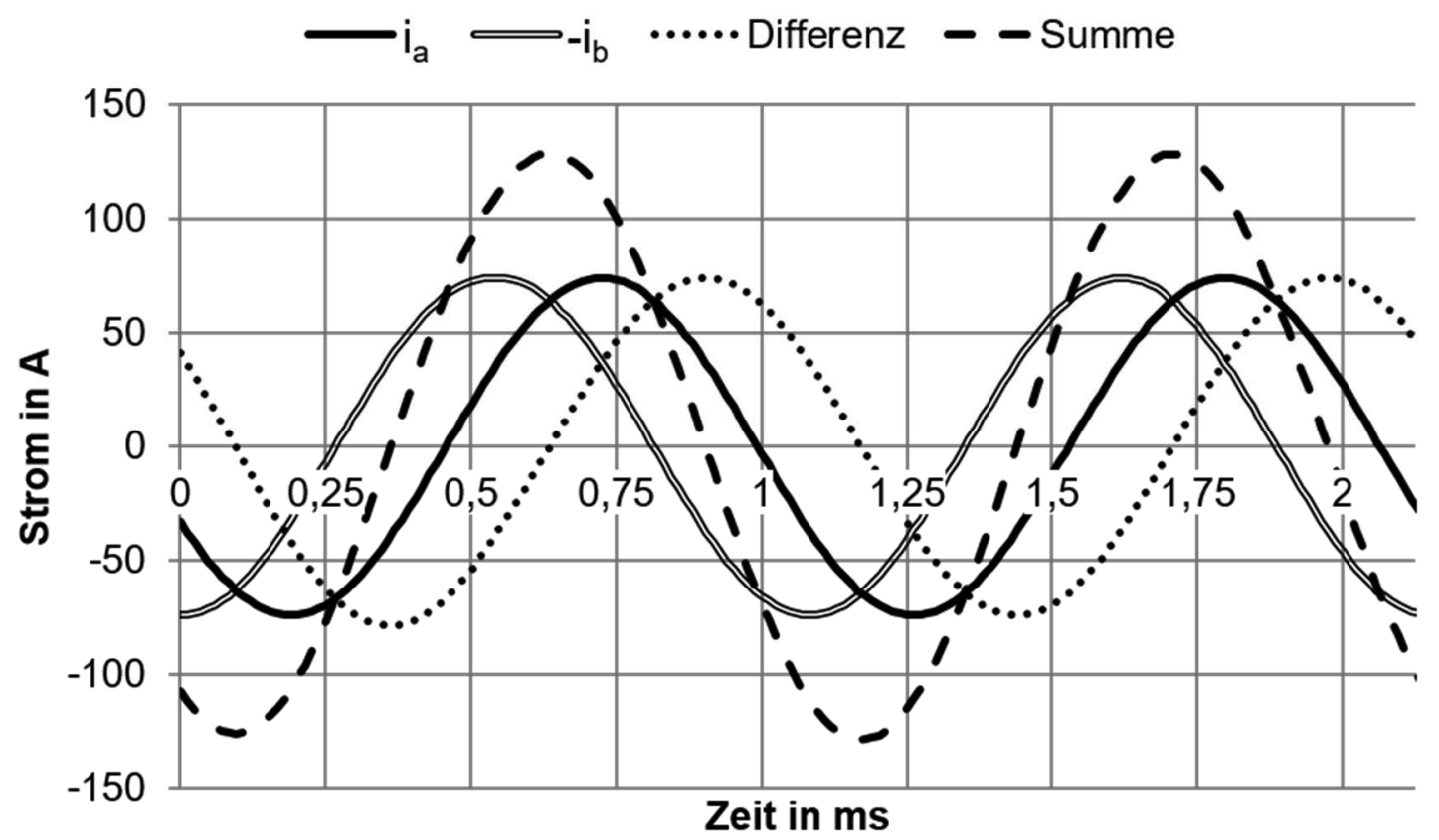

Abbildung 6-2: Stromverlauf in den Spulenseiten

Zusätzlich sind die Summe der Spulenseitenströme sowie die Differenz zwischen den beiden Spulenseitenströmen aufgetragen. Die Amplitude des für die Nut wirksamen Gesamtstroms $\hat{\imath}_{\overline{A B}}(\theta)$ verringert sich durch die Phasenverschiebung der Spulenströme. Da der Strom die Ursache der betrachteten Stromverdrängung ist, liegt die Annahme nahe, dass sich in der Folge auch die Stromverdrängung reduziert, wie es sich aus Mosebachs Untersuchungen [29] ergibt. Zur Veranschaulichung lässt sich dies anhand des vereinfachten Ersatzschaltbilds in Abbildung 6-3 betrachten: 


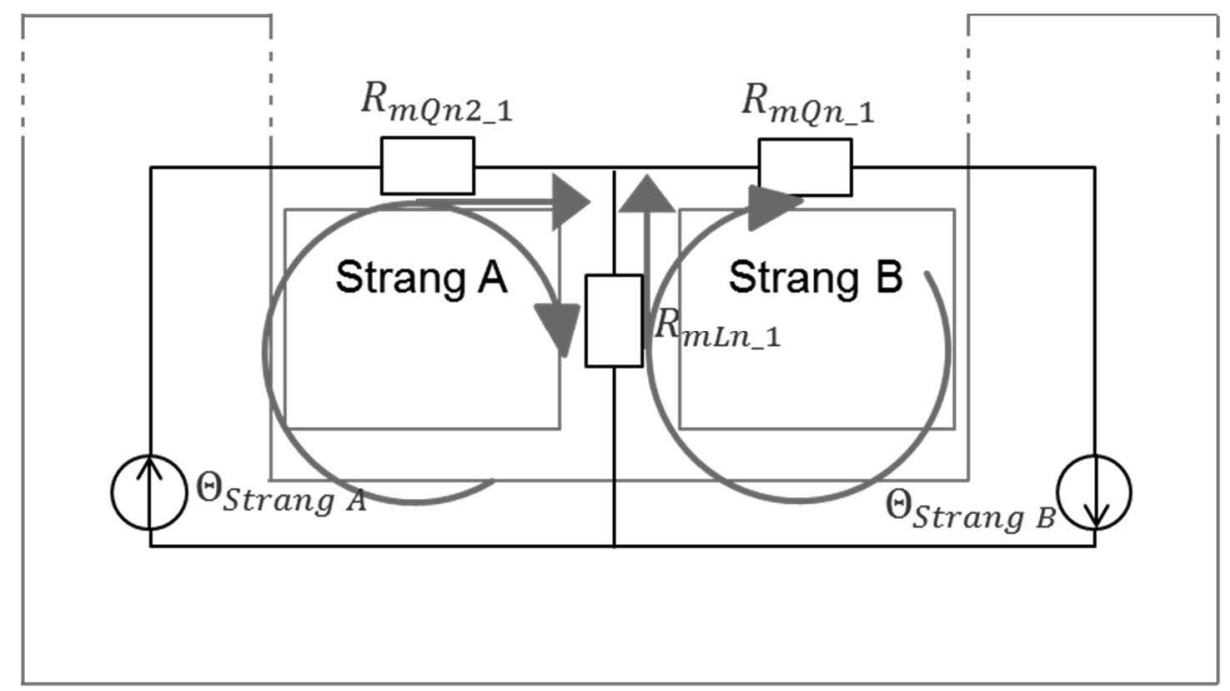

Abbildung 6-3: Ersatzschaltbild der magnetischen Wirbelfeldentstehung bei zwei nebeneinander in der Nut liegenden Spulenseiten zum Zeitpunkt des maximalen Gesamtstroms $\hat{\imath}_{\overline{A B}}(\theta)$

Nimmt man beispielsweise den Zeitpunkt, zu dem der für die Nut wirksame Gesamtstrom $\hat{\imath}_{\overline{A B}}(\theta)$ sein Maximum annimmt, so fließt in beiden Strängen der gleiche Strom. Dadurch sind die Verhältnisse vergleichbar mit dem Fall, dass nur ein Strang in der Nut liegt. Jedoch ist, wie schon erwähnt, die Amplitude des für die Nut wirksamen Gesamtstroms aufgrund der Phasenverschiebung geringer. Der Längsfeldanteil des Strangs A ist dem Längsfeldanteil des Strangs B entgegengerichtet. Dadurch hebt sich dieser in Nutmitte nahezu auf und es gibt keine relevante Längsfeldkomponente.

Die Amplitude der Stromdifferenz zwischen den beiden Strängen in Abbildung 6-2 wird hier als $\hat{\imath}_{\triangle A B}(\theta)$ bezeichnet. Betrachtet man den Zeitpunkt, zu dem die Stromdifferenz ihr Maximum annimmt, so fällt auf, dass das von den beiden Strängen erzeugte magnetische Querfeld zu diesem Zeitpunkt aufgrund des unterschiedlichen Vorzeichens entgegengerichtet ist, wie es aus Abbildung 6-4 hervorgeht:

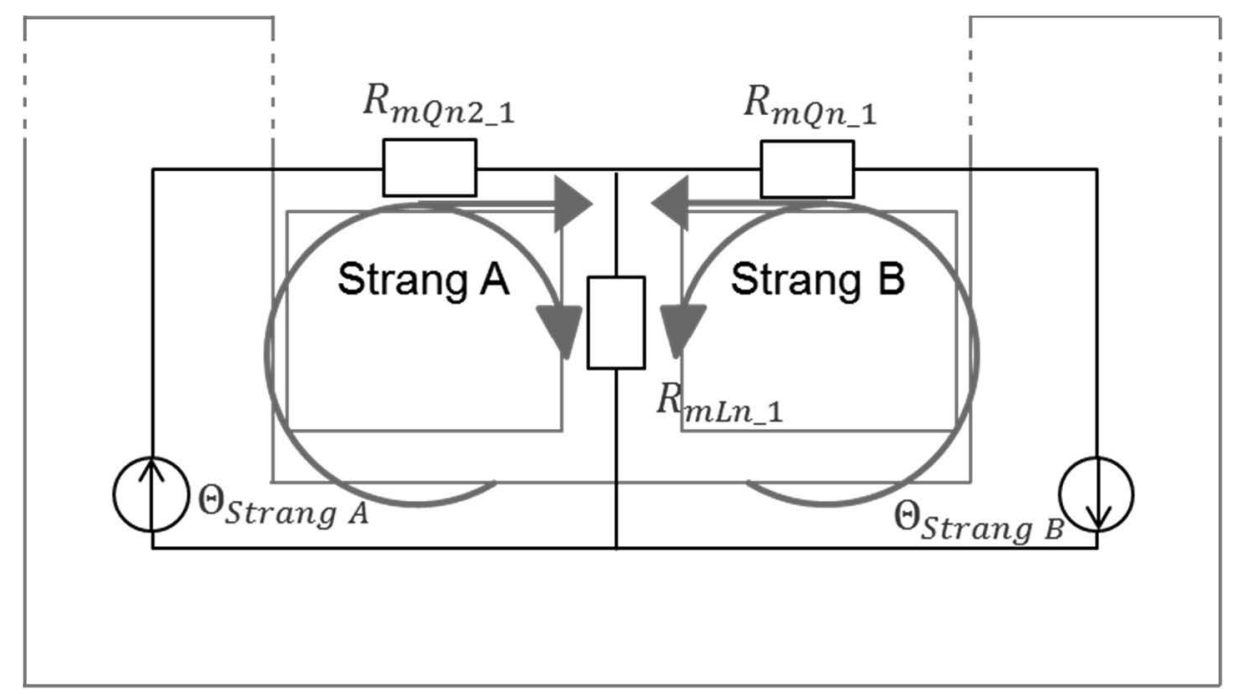

Abbildung 6-4: Ersatzschaltbild der magnetischen Wirbelfeldentstehung bei zwei nebeneinander in der Nut liegenden Strängen zum Zeitpunkt der maximalen Stromdifferenz zwischen den Spulenseitenströmen $\hat{\iota}_{\triangle A B}(\theta)$ 
Dadurch hebt sich die Querfeldkomponente in diesem Punkt auf, und es gibt kein relevantes Querfeld, sondern nur ein Längsfeld. Alle anderen auftretenden Strompaarungen liegen zwischen den beiden illustrierten Grenzszenarien und weisen sowohl Querfeld- als auch Längsfeldanteile auf. Dabei hängt der Anteil von Längsfeld und Querfeld von der Phasenverschiebung zwischen den beiden Strängen ab. Mit zunehmender Phasenverschiebung sollte sich der Querfeldanteil verringern, während der Längsfeldanteil steigt.

\subsection{Betrachtung mittels Finite-Elemente-Rechnungen}

Im vorherigen Abschnitt ergab sich aus den Vorüberlegungen die Annahme, dass nebeneinander in der Nut liegende Spulenseiten unterschiedlicher Stränge das Nutfeld und damit auch die Stromverdrängung in den Leitern beeinflussen. Dies wird in diesem Abschnitt mit Hilfe von Finite-Elemente-Rechnungen untersucht. Dazu wird eine Anpassung an dem in Kapitel 4 beschriebenen Grundmodell vorgenommen. In dieses werden zwei zu verschiedenen Strängen gehörende nebeneinander in der Nut liegende Spulenhälften integriert, wie es schematisch in Abbildung 6-1 dargestellt ist. Die Stränge werden zunächst mit einem um $60^{\circ}$ verschobenen Strom gespeist.

\subsubsection{Einfluss auf das Nutfeld}

Im ersten Schritt wird das Nutfeld mit der in Kapitel 4 beschriebenen Methodik ausgewertet. In Abbildung 6-5 ist exemplarisch das Längsfeld an der Position „Längsfeld 1" aufgetragen, die in Abbildung 6-1 festgelegt wurde:

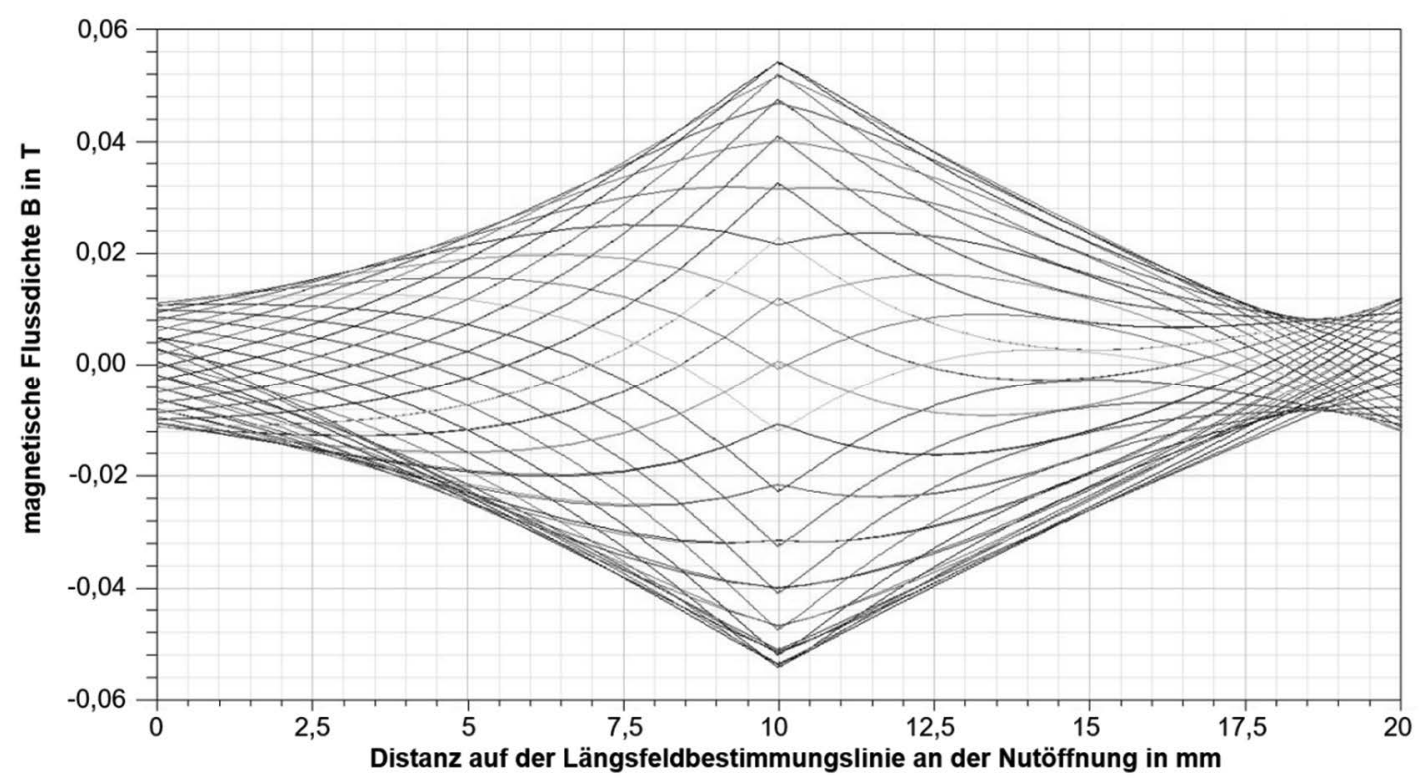

Abbildung 6-5: Durch nebeneinanderliegende Spulenseiten in der Nut mit einer Phasenverschiebung des Stroms von $60^{\circ}$ entstehendes Längsfeld an der Position Längsfeld 1 (siehe Abbildung 6-1)

Dabei ergibt sich ein charakteristischer Anstieg des Längsfelds von den Zahnrändern zur Nutmitte. Dort erreicht das Längsfeld seine höchste Amplitude. Dieses Verhalten passt zu den Vorüberlegungen aus Abschnitt 6.1. Des Weiteren zeigt sich anhand der Abbildung 6-6, dass sich das Querfeld gegenüber einer phasengleichen Bestromung der Leiter verringert: 


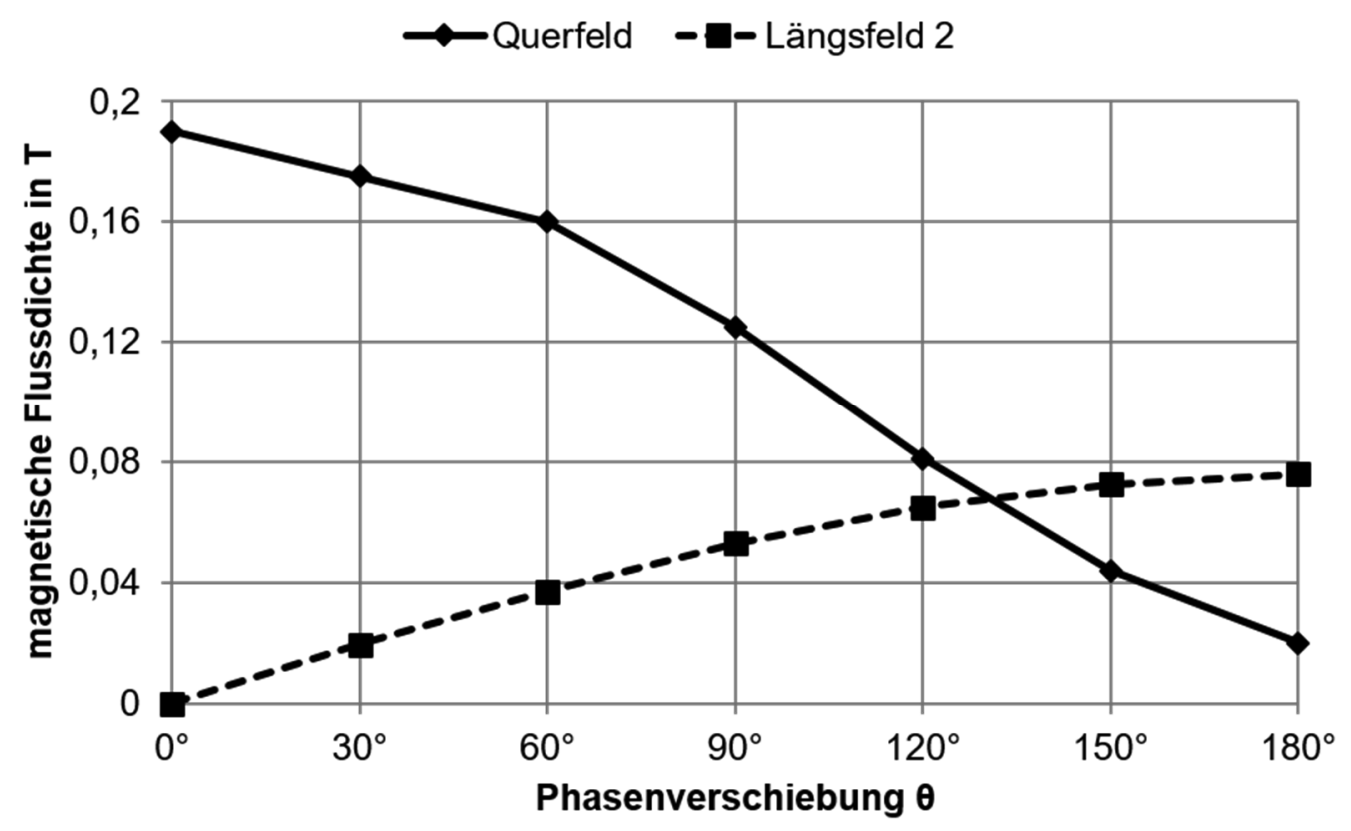

Abbildung 6-6: Beeinflussung des Nutfelds durch die phasenverschobene Bestromung von Zahnspulen

In der Darstellung ist jeweils die Amplitude des Längsfelds an der Position "Längsfeld 2" und des Querfelds für verschiedene Phasenverschiebungen des Stroms aufgetragen. Es ist zu erkennen, dass die Längsfeldkomponente mit zunehmender Phasenverschiebung zunimmt, während die Querfeldkomponente immer weiter abnimmt. Auch dieses Verhalten deckt sich mit den Vorüberlegungen aus Abschnitt 6.1.

\subsubsection{Einfluss auf die Stromverdrängung}

In einem weiteren Schritt wird die Auswirkung der zwei nebeneinander in der Nut liegenden Spulenseiten auf die Stromverdrängung im Leiter untersucht. Dazu wird die in Abschnitt 6.2.1 verwendete Zahnspulenanordnung bei $933 \mathrm{~Hz}$ mit einer Phasenverschiebung von $60^{\circ}$ berechnet. Der entstehende $k_{k}$-Verlauf für den Strang $A$ wird dem in Kapitel 4 betrachteten Fall von nur einem Strang in der Nut gegenübergestellt. Die Betrachtung wird für einen nicht sättigenden Eisenkreis vorgenommen. Das Ergebnis ist in Abbildung 6-7 zu sehen: 


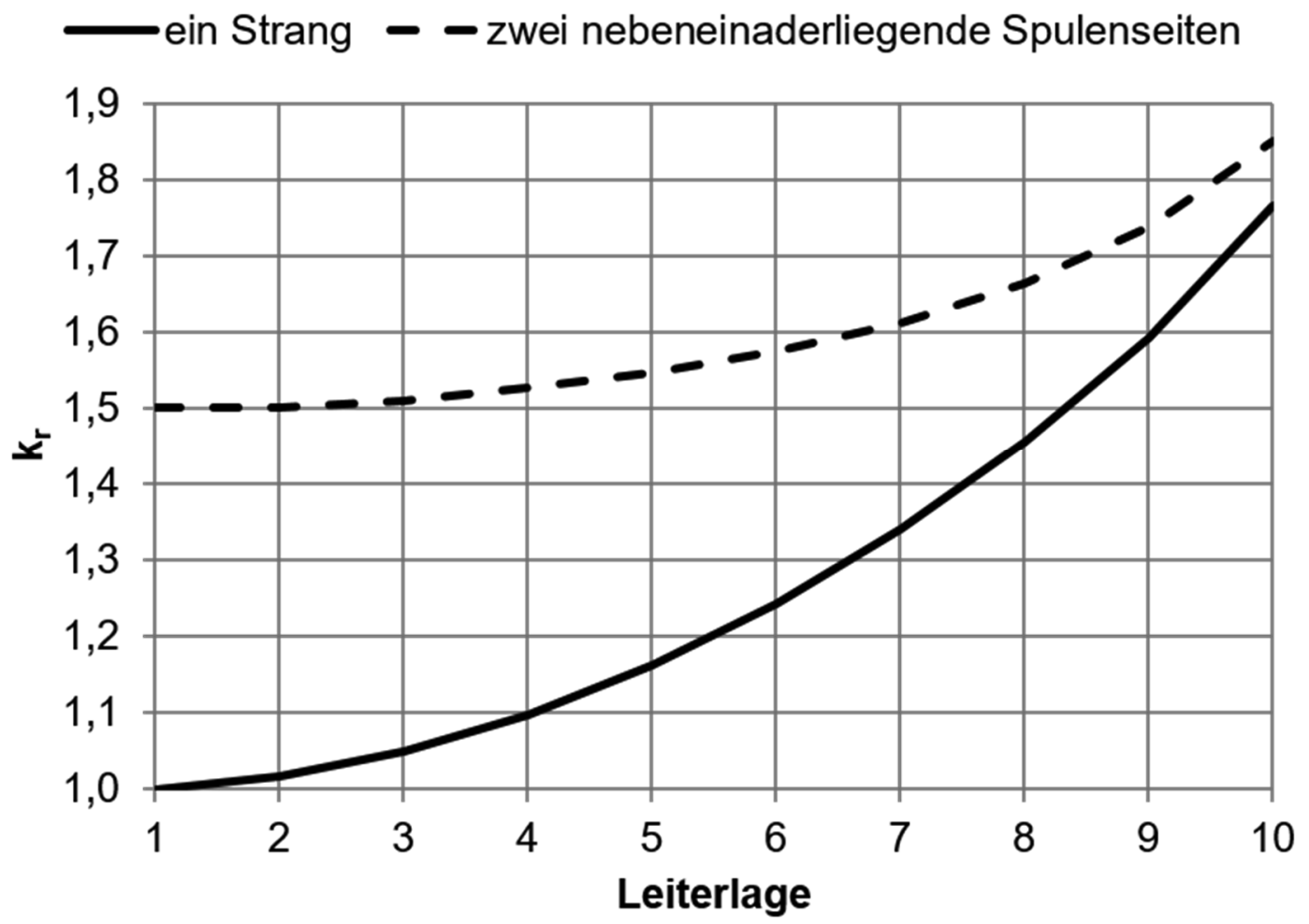

Abbildung 6-7: Gegenüberstellung der $k_{r}$-Faktoren für eine Strang und zwei zu verschiedenene Strängen gehörenden Spulenseiten, die nebeneinander in der Nut liegen

Für zwei nebeneinander in der Nut liegende Spulenseiten ergibt sich ein deutlicher Anstieg des $k_{r}$-Verlaufs - insbesondere im unteren Nutbereich. Dieser Anstieg lässt sich mit Mosebachs Ansatz [29] nicht erklären. Denn nach diesem führt ein phasenverschobener Strom in der Nut stets zu einem reduzierten $k_{r}$-Verlauf.

Nach den Vorüberlegungen ist der Anstieg auf die zusätzlich entstehende Längsfeldkomponente zurückzuführen. Dies wird mit Hilfe weiterer Berechnungen tiefergehend untersucht. Dazu wird das Finite-Elemente-Modell gemäß Abbildung 6-8 modifiziert.

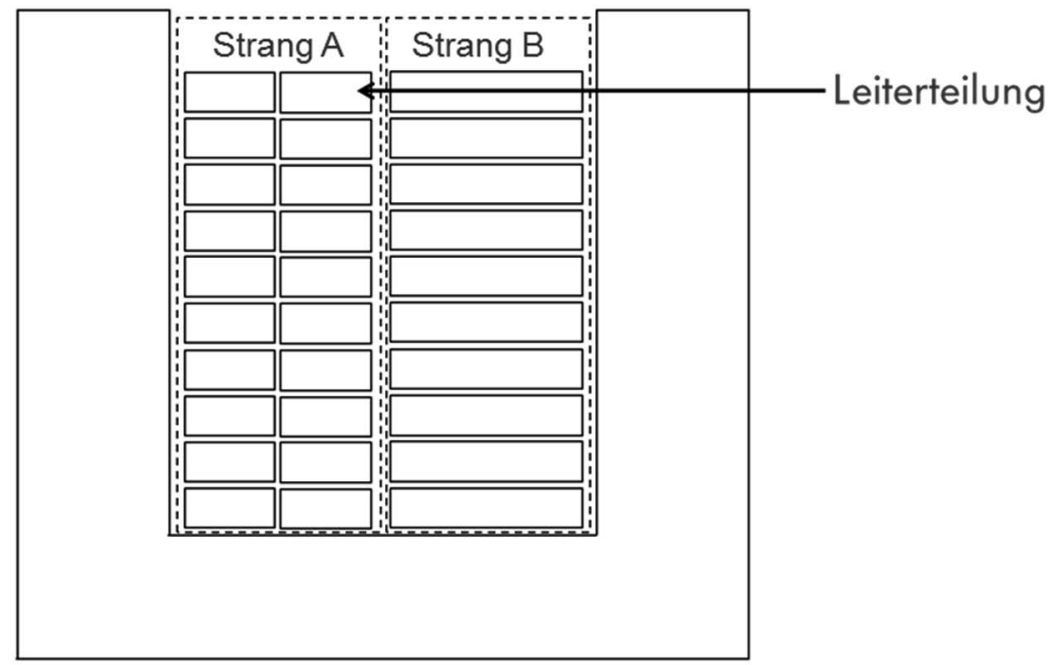

Abbildung 6-8: Exemplarische Teilung der Leiter einer Spulenseite in zwei Teilleiter 
Die Leiter des betrachteten Strangs A werden in der Mitte geteilt, und die linken und rechten Teilleiter werden jeweils nur noch mit halbem Strom gespeist. Durch die verringerte Breite werden die Leiter weniger anfällig für Stromverdrängung durch Längsfelder. In einem weiteren Schritt werden die Leiter in vier nebeneinanderliegende Teilleiter aufgeteilt. Für diese Varianten werden ebenfalls die $k_{r}$ Faktoren bestimmt und den zuvor berechneten Verläufen gegenübergestellt:

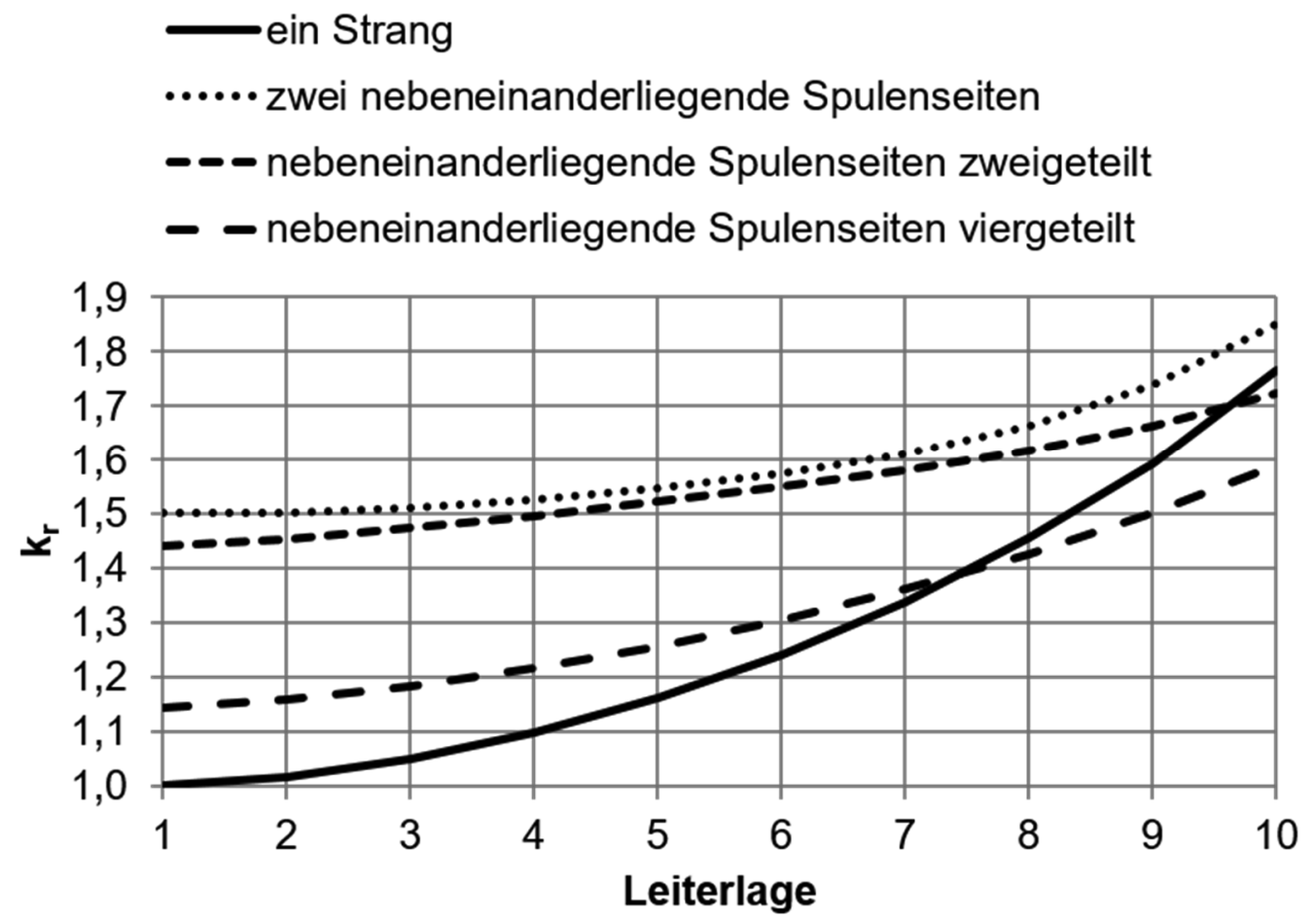

Abbildung 6-9: Gegenüberstellung der $k_{r}$-Faktoren für verschiedene Fälle von nebeneinanderliegenden Spulenseiten in einer Nut

Aus Abbildung 6-9 ist ersichtlich, dass die Teilung der Leiter in zwei Leiter halber Breite sich kaum auf den Verlauf des Widerstandserhöhungsfaktors auswirkt; er liegt fast gleichauf mit der ungeteilten Variante. Dies gilt auch für das Querfeld. So illustriert Richter in seinem Buch [50] den Fall eines Leiters, der in der Höhe in zwei Teilleiter aufgeteilt wird. Dabei steigt der mittlere $k_{r}$-Faktor signifikant an. Auch bei einer weiteren, dreigeteilten Variante sinkt der Wert nur geringfügig gegenüber der zweigeteilten Variante ab. Die Ursache ist, dass die Stromverdrängung in den abgetrennten Teilleitern nicht nur durch den eigenen Stromfluss verursacht wird, sondern auch durch den Stromfluss in den anderen Teilleitern. Durch diesen zusätzlichen Effekt kann der mittlere Widerstandserhöhungsfaktor zunächst ansteigen.

Betrachtet man in Abbildung 6-9 die viergeteilte Variante, so ist zu sehen, dass der Verlauf des $k_{r}$-Faktors über die Leiterlagen deutlich absinkt. Dies deutet daraufhin, dass das hohe Niveau des Verlaufs der ungeteilten, zweisträngigen Variante durch Längsfeldanteile bewirkt wird. In den oberen Leiterlagen sinkt der Widerstandserhöhungsfaktor der viergeteilten Variante sogar unter den Verlauf bei nur einem Strang in der Nut. Hier macht sich die Verringerung der Querfeldkomponente durch die zwei nebeneinanderliegenden Spulenseiten in der Nut bemerkbar, wie sie in Abschnitt 6.1 und bei Mosebach [29] beschrieben wird. In Abbildung 6-10 ist das Ergebnis des Mosebach-Ansatzes aufgetragen. 


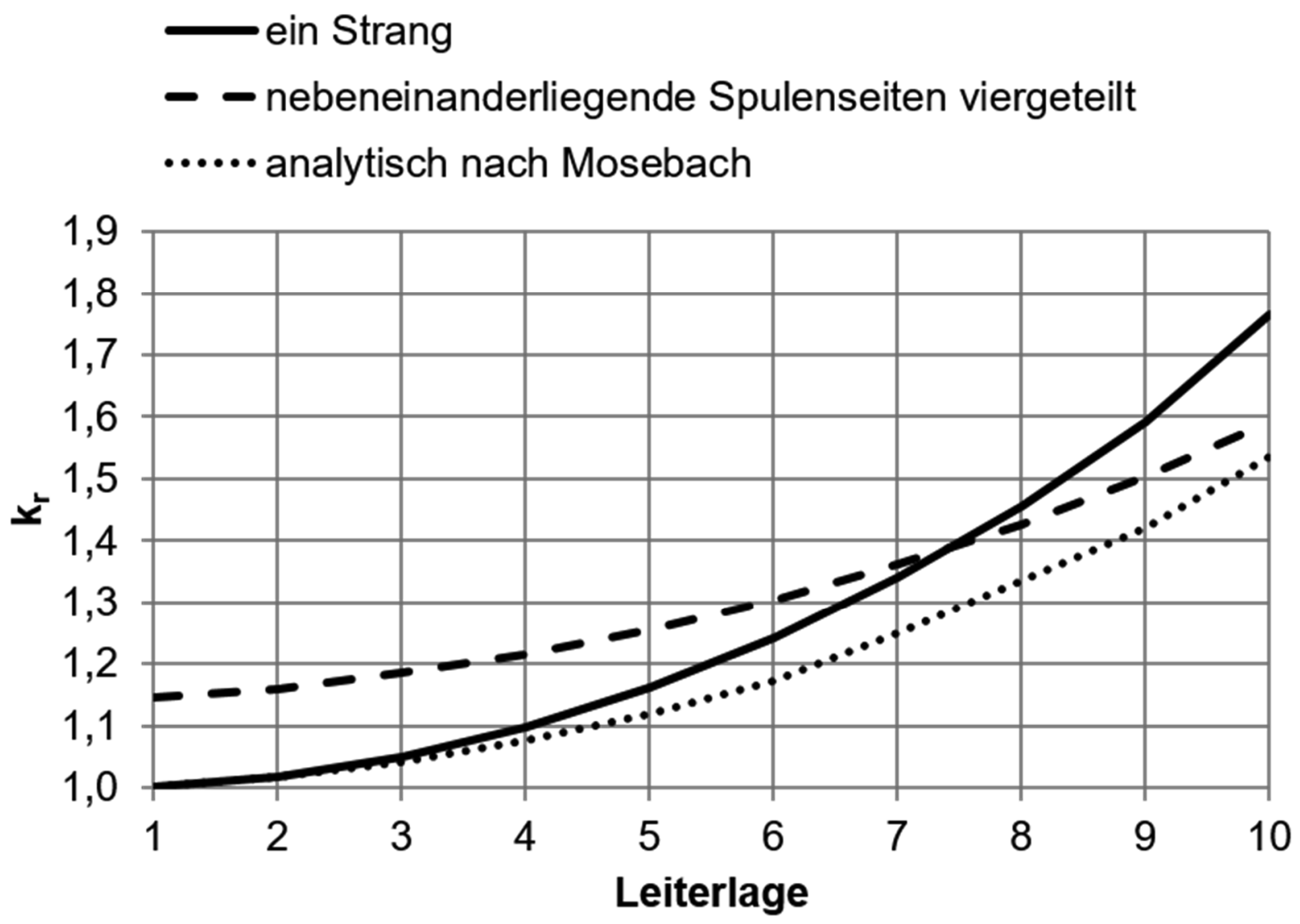

Abbildung 6-10: Gegenüberstellung der $k_{r}$-Faktoren für zwei nebeneinanderliegende Spulenseiten in einer Nut, für eine viergeteilte, mittels FEM berechnete Variante und eine analytisch nach Mosebach [29] berechnete Variante

Es ist zu erkennen, dass die $k_{r}$-Werte der analytisch nach Mosebach [29] berechneten Variante in den oberen Leiterlagen gegenüber dem Fall von nur einer Spulenseite in der Nut verringert werden. Dies ist auf die in den Abschnitten zuvor beschriebene verminderte Amplitude der Summe der Spulenseitenströme zurückzuführen. Darüber hinaus zeigt Abbildung 6-10, dass sich die Ergebnisse der Finite-ElementeBerechnung durch die seitliche Teilung der Leiter zunehmend der Berechnung nach Mosebach annähern.

Zusammenfassend kann festgehalten werden, dass die hier vorgestellten FiniteElemente-Berechnungen die von Mosebach beschriebene Methode für schmale Leiter stützen. Jedoch deutet sich an, dass die Anordnung von zwei zu verschiedenen Strängen gehöhrenden, nebeneinander in der Nut liegenden Spulenseiten neben der von Mosebach berücksichtigten Verringerung des Querfelds auch die Entstehung einer Längsfeldkomponente bewirkt. Diese Längsfeldkomponente ist in der Literatur bisher nicht beschrieben, hat aber bei breiten Leitern einen signifikanten Einfluss auf den Widerstandserhöhungsfaktor $k_{\text {r. }}$. Dies erklärt die in der Einleitung des Kapitel 6 beschriebenen Abweichungen in Bezug auf flache, breite Leiter. 


\subsubsection{E-Maschine mit gegossenen Spulen}

Analog zu Kapitel 5 soll nun betrachtetet werden, wie sich die Anordnung von zwei Spulenseiten unterschiedlicher Stränge nebeneinander in der Nut auf eine real ausgelegte elektrische Maschine auswirken. Dazu wird das Finite-Elemente-Modell der aus Abschnitt 5.2.3 bekannten elektrischen Maschine mit gegossenen Spulen und nicht sättigendem Eisenkreis verwendet. Es wird für die Betrachtung in demselben Betriebspunkt berechnet wie für die Untersuchung in Abschnitt 5.2.3. In den bisherigen Simulationen dieser elektrischen Maschine waren stets die Folgen der nebeneinander in den Nuten liegenden Spulenseiten unterschiedlicher Stränge enthalten. Um die Größenordnung der Auswirkung zu ermitteln, wird an dieser Stelle eine Berechnung durchgeführt, bei der die Spulenseiten in der gleichen elektrischen Maschine als übereinanderliegend angenommen werden.

Der nicht sättigende Eisenkreis wird beibehalten, um zu bestimmen, inwieweit der sättigende Eisenkreis und die nebeneinanderliegende Anordnung der Spulenseiten in den Nuten eine Abweichung von den Ergebnissen der analytischen Berechnung nach Abschnitt 2.1.4 bewirken. Die entsprechenden $k_{r}$-Faktoren der Berechnung sind in Abbildung 6-11 aufgetragen.

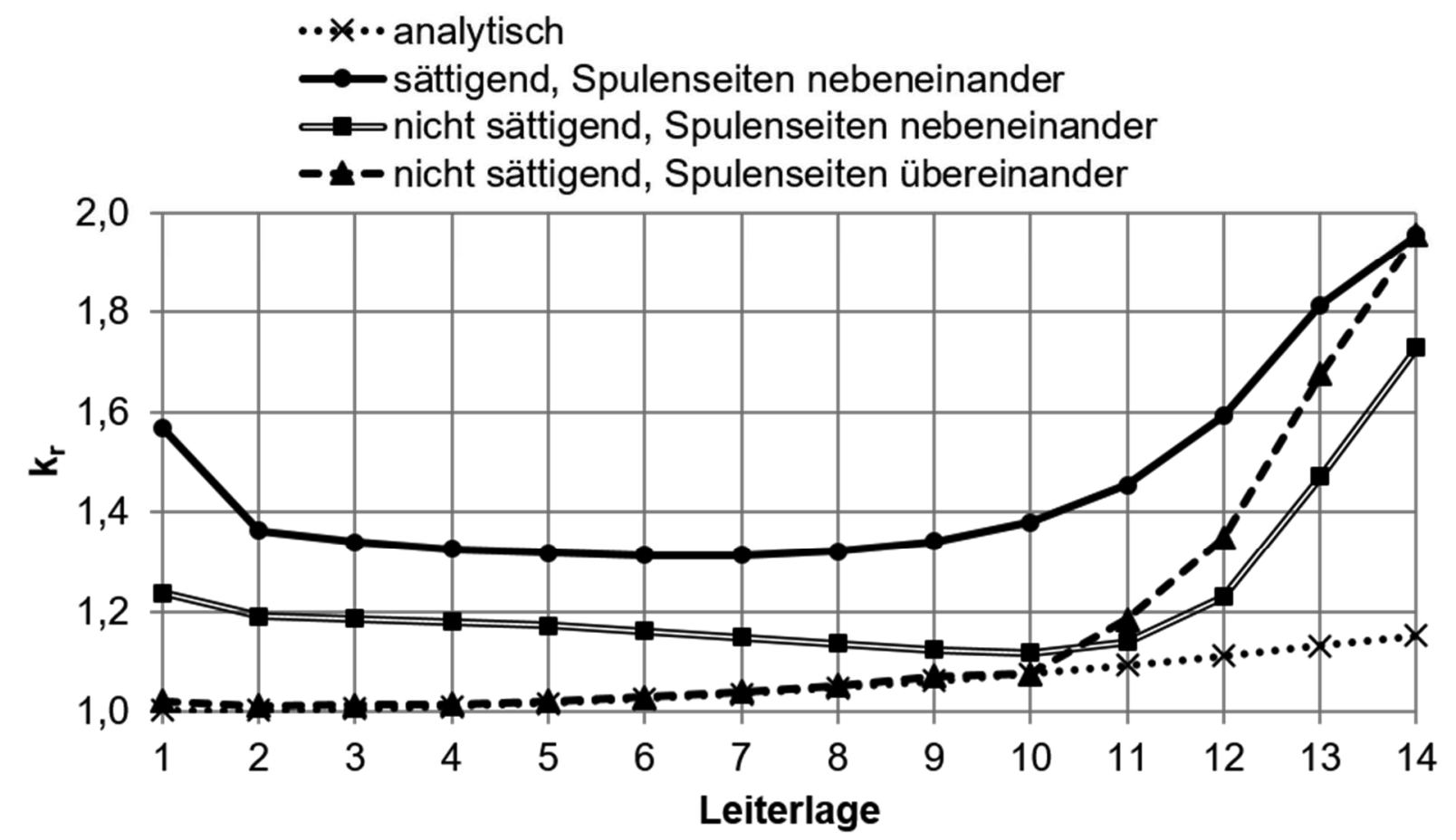

Abbildung 6-11: Gegenüberstellung der analytisch ermittelten $k_{r}$-Faktoren nach Abschnitt 2.1.4 zu drei mittels FEM berechneten Varianten

Zunächst ist noch einmal die Erkenntnis aus Abschnitt 5.2.3 aufgetragen, dass sich der $k_{r}$-Verlauf durch die Verwendung eines fiktiven, nicht sättigenden Blechs in seinem Niveau signifikant reduziert. Der mittlere $k_{r}$-Wert sinkt von zuvor 1,46 auf 1,23. Vergleicht man den $k_{r}$-Verlauf mit dem analytisch nach Abschnitt 2.1.4 berechneten Verlauf, so fällt auf, dass trotz des nicht sättigenden Blechs die $k_{r}$-Werte im unteren und im oberen Nutbereich noch immer deutlich abweichen. Die Abweichung im oberen Nutbereich lässt sich durch die Wechselwirkung mit den Permanentmagneten des Rotors erklären. Doch für die Abweichung im unteren Nutbereich findet sich im Stand 
der Technik keine plausible Erklärung. Betrachtet man nun die Variante mit nicht sättigendem Blech und zusätzlicher übereinanderliegender Spulenseitenanordnung, so ist der $k_{r}$-Verlauf bei dieser in den unteren und mittleren Leiterlagen nahezu deckungsgleich mit den Ergebnissen der analytischen Berechnung. Gleichzeitig steigt jedoch der Widerstandserhöhungsfaktor ab der elften Leiterlage an. Dies ist auf die Stärkung der Querfeldkomponente zurückzuführen. Der mittlere $k_{r}$-Faktor reduziert sich dadurch jedoch nur leicht von 1,23 auf 1,18 und nähert sich somit weiter den Ergebnissen der analytischen Berechnung mit einem Wert von 1,05 an.

Zusammenfassend lässt sich festhalten, dass die in der Nut nebeneinanderliegende Spulenseitenanordnung unterschiedlicher Stränge bei einer real ausgelegten Maschine mit breiten, flachen Leitern einen signifikanten Einfluss auf die Stromverdrängung hat. Wesentliche Ursachen des Anstiegs der $k_{r}$-Faktoren im unteren Nutbereich sind bei der betrachteten Maschine somit die Sättigung des magnetischen Kreises und die nebeneinanderliegende Anordnung von zwei zu verschiedenen Strängen gehörenden Spulenseiten in der Nut.

\subsection{Ableitung analytischer Erkenntnisse}

In diesem Abschnitt wird ein neuentwickeltes analytisches Berechnungsverfahren vorgestellt, das in der Lage ist, die Auswirkungen von zwei nebeneinander in der Nut angeordneten, zu verschiedenen Strängen gehörenden Spulenseiten auf die Stromverdrängung zu bestimmen. Dieses basiert auf den Vorüberlegungen aus Abschnitt 6.1 und den Betrachtungen mittels Finite-Elemente-Berechnungen aus Abschnitt 6.2.

In den Vorüberlegungen wurde aufgezeigt, dass die Amplitude des für die Nut wirksamen Gesamtstroms $\hat{\imath}_{\overline{A B}}(\theta)$ durch die nebeneinanderliegende Anordnung der Spulenseiten in einer Nut verringert wird, was die Querfeldkomponente des Nutfelds reduziert. Gleichzeitig wächst die Längsfeldkomponente mit zunehmender Differenz zwischen den beiden Strangströmen $\hat{\iota}_{\triangle A B}(\theta)$ an. Abbildung 6-12 zeigt, wie sich die Amplitude des für die Nut wirksamen Gesamtstroms $\hat{l}_{\overline{A B}}(\theta)$ und die Amplitude der Stromdifferenz zwischen den Strömen $\hat{\imath}_{\triangle A B}(\theta)$ mit zunehmender Phasenverschiebung der speisenden Ströme verändert. 


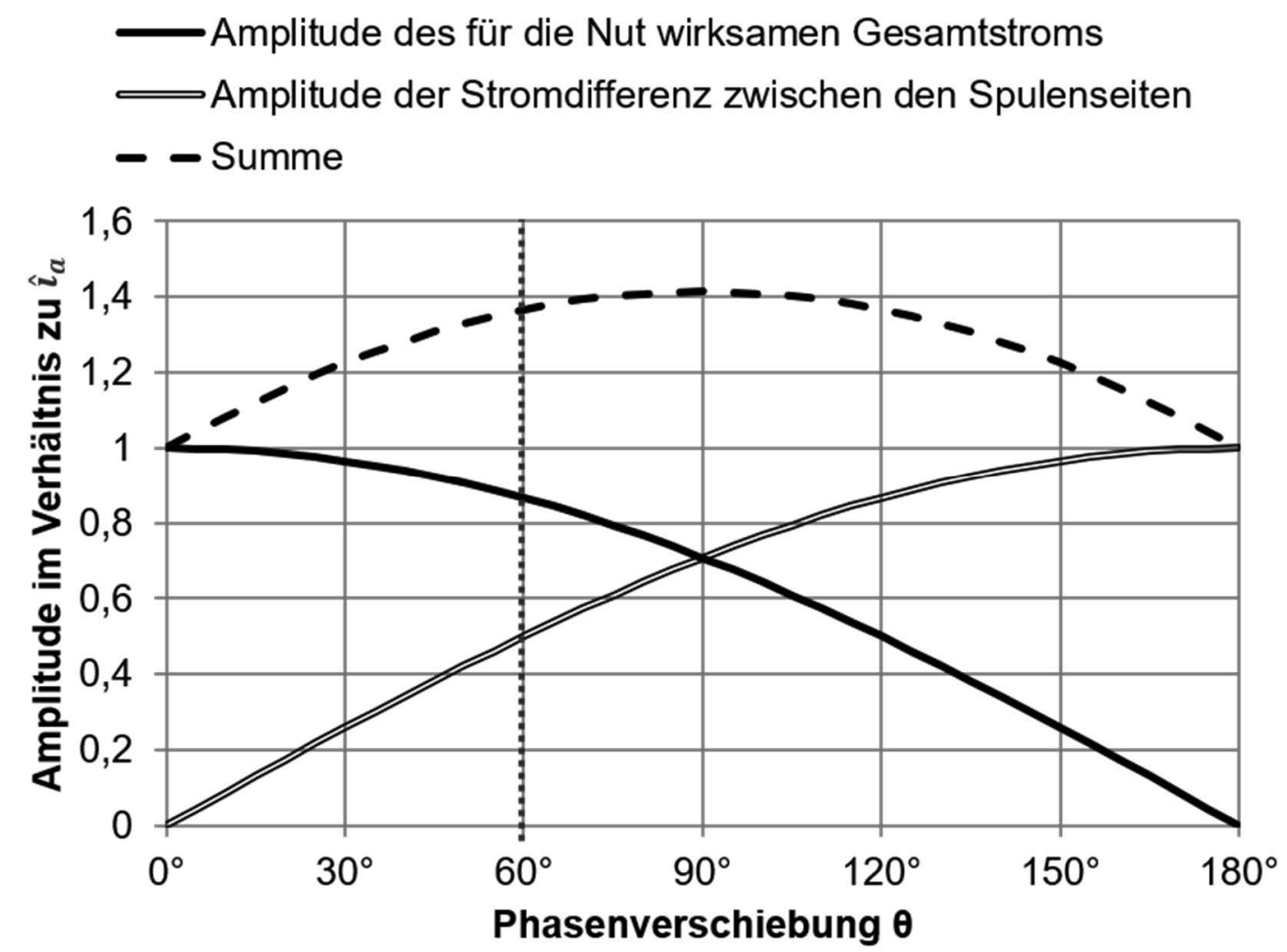

Abbildung 6-12: Amplitudenverhältnis des für die Nut wirksamen Gesamtstroms und der Stromdifferenz zwischen zwei Spulenseiten für verschiedene Phasenverschiebungswinkel als Grundlage der Verminderungsfaktoren $a_{\overline{A B}} q f$ und $a_{\triangle A B l f}$ für den entwickelten analytischen Ansatz

Die betrachteten Amplituden beeinflussen nach Abschnitt 6.1 den jeweiligen Anteil von Längsfeld- und Querfeldkomponente. Die Größen, die sich allgemein für die Phasenverschiebung zwischen zwei Sinusfunktionen kalkulieren lassen, werden für die analytische Ermittlung der durch Quer- und Längsfeldkomponente entstehenden Stromverdrängung herangezogen. Mit innen werden die Verminderungsfaktoren $a_{\overline{A B} s q f}$ und $a_{\triangle A B s l f}$ gemäß den Gleichungen (6-1) und (6-2) bestimmt:

$$
\begin{aligned}
a_{\overline{A B} q f} & =\frac{\hat{\imath}_{\overline{A B}}(\theta)}{\hat{\imath}_{\overline{A B}}(\theta)+\hat{\imath}_{\Delta A B}(\theta)} \\
a_{\triangle A B l f} & =\frac{\hat{\imath}_{\triangle A B}(\theta)}{\hat{\imath}_{\overline{A B}}(\theta)+\hat{\imath}_{\Delta A B}(\theta)} .
\end{aligned}
$$

Anders als bei der magnetischen Sättigung des Eisenkreises handelt es sich bei diesen Verminderungsfaktoren in der Regel um konstante Werte, da für gewöhnlich feste Phasenverschiebungen zwischen den Strömen zweier Stränge bestehen. Jedoch hängt es vom Wickelschema ab, welche Phasenverschiebung in den einzelnen Nuten zwischen den Spulenseitenströmen besteht. Ein weiterer Unterschied zur magnetischen Sättigung ist, wie die Verminderungsfaktoren in die Berechnung des Widerstandserhöhungsfaktors einfließen. Denn sie gehen nicht in die Ermittlung des 
Reduktionsfaktors $a$ ein. Dieser wird für den Querfeldanteil gemäß Gleichung (2-22) und für den Längsfeldanteil berechnet gemäß

$$
\alpha_{2 l f}=\sqrt{\pi f \mu_{0} \kappa \frac{h_{l}}{h_{n}}} .
$$

Anschließend werden mittels Gleichung (2-21) für das Querfeld und Gleichung (5-7) für das Längsfeld die reduzierten Leiterhöhen $\beta$ kalkuliert. Mit den reduzierten Leiterhöhen und den Gleichungen (2-19) und (2-20) werden danach die Hilfsfunktion zur Berechnung der Widerstandserhöhungsfaktoren $\varphi(\beta)$ und $\psi(\beta)$ für Quer- und Längsfeld separat errechnet. Ebenso wird der Widerstandserhöhungsfaktor $k_{r}$ im Anschluss mit Gleichung (2-18) gesondert für Quer- und Längsfeld ermittelt. Erst dann kommen die Verminderungsfaktoren zum Einsatz. Mit diesen wird aus den separat für Längsfeld und Querfeld bestimmten Widerstandserhöhungsfaktoren mit Hilfe einer gewichteten Addition der resultierende $k_{r}$-Faktorverlauf berechnet zu

$$
k_{r 2}=a_{\overline{A B} q f} k_{r 2 q f}+a_{\Delta A B l f} k_{r 2 l f} .
$$

Gleichung (6-4) gilt in dieser Form jedoch nur, wenn in jeder Nut die gleiche Phasenverschiebung für die nebeneinanderliegenden, zu verschiedenen Strängen gehörenden Spulenseiten vorliegen. Sonst ist diese Gleichung

$$
\begin{aligned}
k_{r 2} & =\frac{v_{1}}{N}\left(a_{\overline{A B} q f, p p 1} k_{r 2 q f}+a_{\Delta A B l f, p p 1} k_{r 2 l f}\right) \\
& +\frac{v_{2}}{N}\left(a_{\overline{A B} q f, p p 2} k_{r 2 q f}+a_{\Delta A B l f, p p 2} k_{r 2 l f}\right)+\cdots \\
& +\frac{v_{n}}{N}\left(a_{\overline{A B} q f, p p n} k_{r 2 q f}+a_{\Delta A B l f, p p n} k_{r 2 l f}\right) .
\end{aligned}
$$

anzuwenden. Der Grund für dieses Berechnungsverfahren ist, dass anders als bei der magnetischen Sättigung des Eisenkreises nicht zwei unterschiedliche Ursachen für die Stromverdrängung in Längs- und in Querrichtung vorliegen. Dort gab es zum einen die Verminderung des Querfelds durch den steigenden magnetischen Widerstand des magnetischen Kreises und zum anderen das zunehmende Längsfeld aufgrund steigender Zahnentlastung. Beide Effekte verursachen getrennt voneinander eine Stromverdrängung, sodass tatsächlich zwei überlagerte Wirbelströmungen addiert werden müssen. Bei zwei nebeneinander in der Nut liegenden, zu verschiedenen Strängen gehörenden Spulenseiten gibt es nur eine Ursache für Stromverdrängung, allerdings wird die Richtung des Nutfelds durch die Phasenverschiebung zwischen den beiden Spulenströmen in der Nut beeinflusst. Die Verminderungsfaktoren definieren hier, wie hoch der Anteil der jeweiligen Komponente ist.

Das Verfahren wurde für verschiedene Phasenverschiebungen auf die in Abbildung 6-1 dargestellte Geometrie angewendet. Dabei wurde eine Anpassung der Nutbreite auf $10 \mathrm{~mm}$ vorgenommen, um zu zeigen, dass das Verfahren in der Lage ist, ein Ab- 
nehmen der Stromverdrängung in den oberen Leiterlagen und ein Zunehmen der Stromverdrängung in den unteren Leiterlagen relativ genau abzubilden. Die $k_{r}$ Verläufe der analytischen Berechnung nach dem hier beschriebenen Verfahren werden in Abbildung 6-13 mit den Ergebnissen einer jeweils entsprechenden FiniteElemente-Betrachtung verglichen.

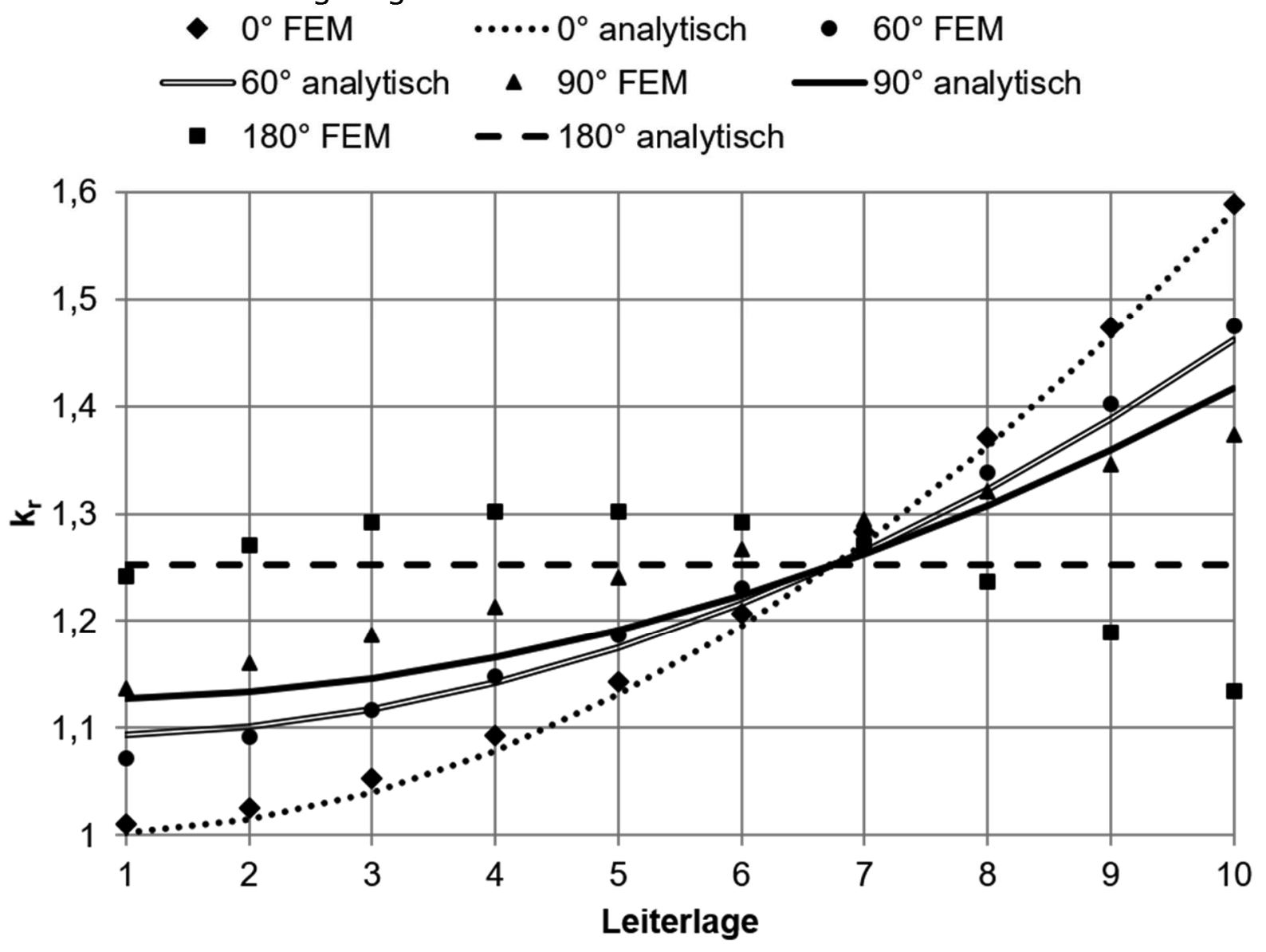

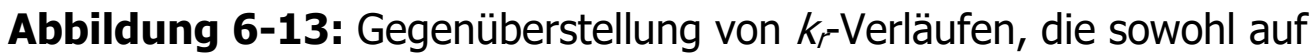
analytischem Wege als auch mittels Finite-Elemente-Simulation für die in Abbildung 6-1 gezeigte Geometrie für verschiedene Phasenverschiebungen des Stroms in den zu verschiedenen Strängen gehörenden, nebeneinanderliegenden Spulenhälften bei einer Stromfrequenz von $933 \mathrm{~Hz}$ und einer Nutbreite von $10 \mathrm{~mm}$ berechnet wurden
\end{abstract}

Dabei ist die analytische Berechnung jeweils als Linie und die Finite-ElementeRechnung als Symbol aufgetragen. Es ist zu erkennen, dass beide Berechnungswege die gleichen Grundtendenzen aufweisen und zu sehr ähnlichen Ergebnissen führen. Bei der untersuchten Leiteranordnung und -geometrie steigt der $k_{r}$-Faktor bis zur siebten Leiterlage mit zunehmender Phasenverschiebung an, während er in den darüber in der Nut liegenden Leitern abnimmt. Erwähnenswert ist, dass die Abweichung des analytischen Ansatzes von der Finite-Elemente-Berechnung bei größeren Phasenverschiebungen zunimmt. Dies ist darauf zurückzuführen, dass die gegenseitige Beeinflussung der nebeneinanderliegenden Teilleiter bei der analytischen Berechnung zunächst vernachlässigt wird. Auch weitere Einflüsse sind nicht auszuschließen. Beispielsweise weichen die Randbedingungen in den oberen Leiterlagen zunehmend von den Annahmen in Abschnitt $6.1 \mathrm{ab}$, da das Joch weiter entfernt ist. 
Bei der betrachteten Leiteranordnung und -geometrie verändert sich der mittlere $k_{r}$-Faktor durch die zunehmend phasenverschobene Bestromung der beiden nebeneinander in der Nut liegenden Spulenseiten kaum. Die durch Stromverdrängung zusätzlich in der Spule auftretenden Verluste bleiben also fast konstant, nur der Ort der Wärmeentwicklung variiert. Hier ist der Vergleich zu dem in Abbildung 6-7 betrachteten Fall von einer $20 \mathrm{~mm}$ breiten Nut interessant. Durch die größere Leiterbreite ist dort die Auswirkung durch zwei zu verschiedenen Strängen gehörende, nebeneinander in der Nut liegende Spulenseiten bereits bei $60^{\circ}$ Phasenverschiebung größer. Der mittlere $k_{r}$-Faktor steigt dort von 1,27 auf 1,6 an. Das verdeutlicht den Einfluss der Nut- und Leiterbreite auf die Stromverdrängung. Der leicht unterschiedliche $k_{r}$-Faktorverlauf der nicht phasenverschobenen Variante in Abbildung 6-7 und Abbildung 6-13 ist auf ein geringfügig verändertes Leiterbreitenzu-Nutbreiten-Verhältnis zurückzuführen.

Zusammenfassend lässt sich sagen, dass sich die Ergebnisse des hier vorgestellten analytischen Verfahrens grundsätzlich mit den Ergebnissen der Finite-ElementeSimulation decken. Es wurde gezeigt, dass durch Zahnspulen ein Längsfeldanteil in der Nut entsteht, der sich insbesondere bei breiten, flachen Leitern in einem signifikanten Anstieg des Widerstandserhöhungsfaktors bemerkbar machen kann. Aus Abbildung 6-11 ist ersichtlich, dass der beschriebene Effekt auch bei real ausgelegten Maschinen eine signifikante Rolle spielen kann. Zusätzlich zeigt sich, dass die nebeneinanderliegende Anordnung von zwei zu verschiedenen Strängen gehörenden Spulenseiten bei der betrachteten Maschine mit gegossenen Spulen ein wesentlicher Faktor ist, der eine Abweichung von der analytischen Berechnung nach Abschnitt 2.1.4 verursacht. 


\section{Wechselwirkung mit dem Luftspaltfeld}

In Kapitel 3 wird für eine elektrische Maschine mit gegossenen Spulen festgestellt, dass Berechnungen mittels Finite-Elemente-Methode und Messungen am Prüfstand zu ähnlichen $k_{r}$-Faktoren führen. Im Vergleich dazu weichen analytische Berechnungen nach Abschnitt 2.1.4 signifikant ab. Aus Kapitel 5 und 6 ergibt sich, dass die Vernachlässigung der Sättigung des magnetischen Kreises und die nebeneinanderliegende Anordnung von zwei zu verschiedenen Strängen gehörenden Spulenseiten in einer Nut wesentliche Ursachen für die Abweichungen der analytischen Berechnung sind. Jedoch lassen sich durch die beiden genannten Einflüsse nur die Abweichungen

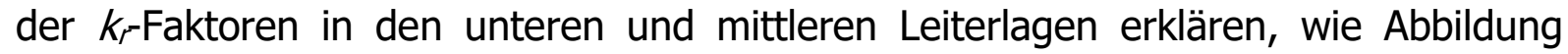
6-11 zeigt. In diesem Kapitel werden nun die Abweichungen in den oberen Leiterlagen behandelt. Es gibt zahlreiche Hinweise, dass das in die Nut eindringende Luftspaltfeld zusätzliche Stromverdrängung in den oberen Leiterlagen verursacht [6] [11] [15] [33] [35] [43] [47]. Vereinzelt finden sich auch analytische Ansätze zur Bestimmung der zusätzlichen Verluste durch das in die Nut eindringende Luftspaltfeld [6] [35] [47]. Diese sind jedoch teilweise widersprüchlich.

In der vorliegenden Arbeit wird kein analytischer Ansatz zur Berücksichtigung der Stromverdrängung durch die Wechselwirkung mit dem Luftspaltfeld entwickelt. Stattdessen wird dargestellt, welche Einflüsse es erschweren, den Zusammenhang analytisch zu bestimmen. Dabei handelt es sich um jene Einflüsse, die die Überlagerung von Rotor- und Statorfeld und damit das Luftspaltfeld bestimmen. Dies sind der Konstantfluss- und der Feldschwächbereich (Abschnitt 2.2.3.2), die Drehmomentbildung aus synchronem Moment und Reluktanzmoment (Abschnitt 2.2.3.1) sowie die Schrägung (Abschnitt 2.2.3.3). Die Überlagerung beeinflusst, inwieweit das Luftspaltfeld in die Nut eindringt, aber auch, wie sehr der Eisenkreis in die magnetische Sättigung getrieben wird. Die Zusammenhänge werden in den folgenden Abschnitten vorgestellt. Zunächst wird der Feldschwächbereich im Zusammenhang mit der Schrägung betrachtet. Danach wird auf den Konstantflussbereich eingegangen. Abschließend wird qualitativ die Drehmomentbildung betrachtet und eine Zusammenfassung des Kapitels gegeben.

\subsection{Einfluss des Feldschwächbereichs und der Schrägung}

Abschnitt 3.6 zeigt, dass das Luftspaltfeld nicht zwingend zu höherer Stromverdrängung führt. In Abbildung 3-11 ist zu sehen, dass im Konstantflussbereich der $k_{r}$ Verlauf ohne Permanentmagnete im unteren Nutbereich signifikant ansteigt. Das hängt mit dem magnetisch gesättigten Eisenkreis zusammen. Genauer wird darauf im nächsten Abschnitt eingegangen. Für die untersuchte elektrische Maschine ergibt sich im Feldschwächbereich ein näherliegendes Verhalten. 


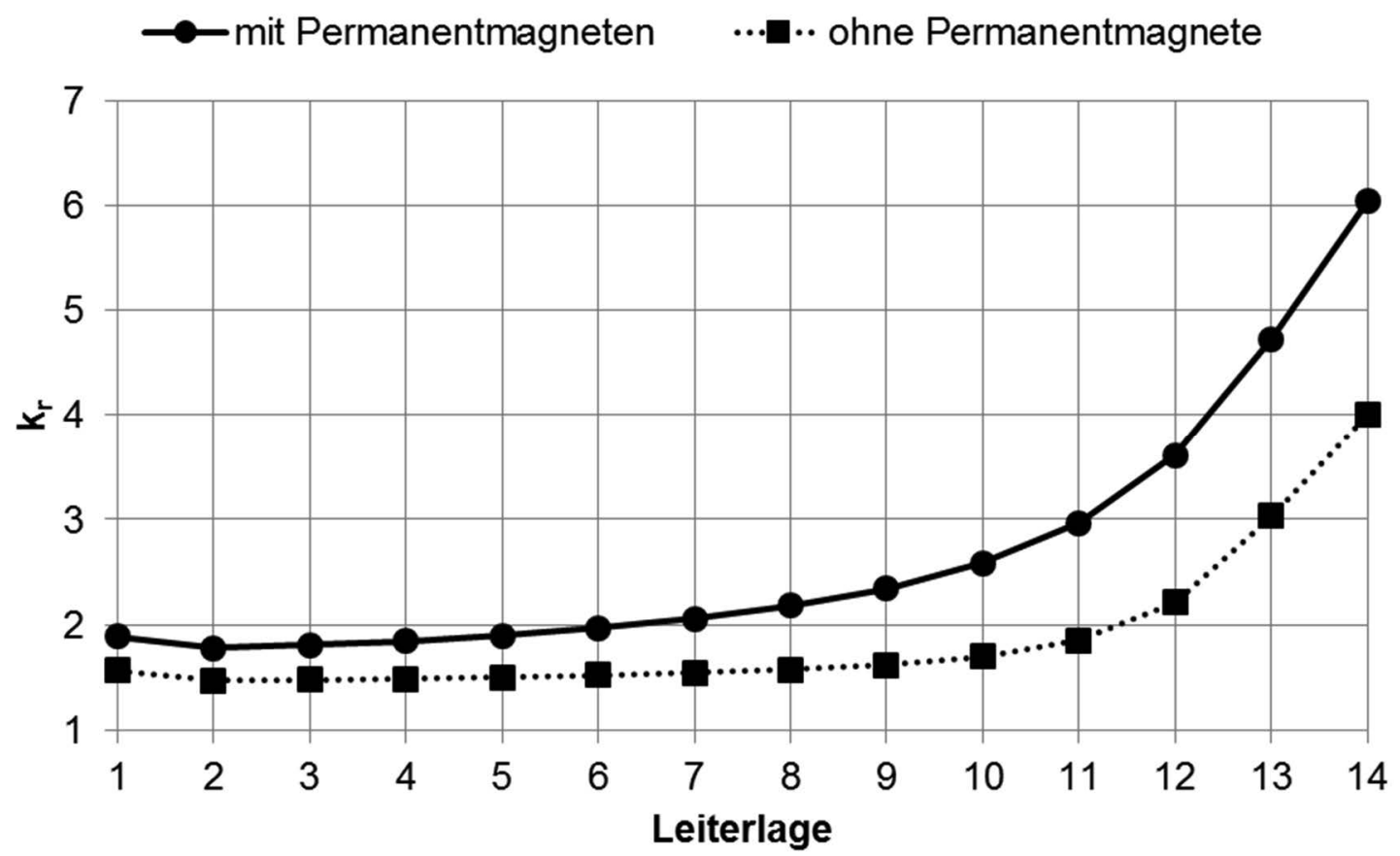

Abbildung 7-1: Gegenüberstellung von $k_{\digamma}$-Verläufen in den Leitern 1 bis 14 (siehe Abbildung 4-3) mit und ohne Permanentmagnete bei einer Frequenz von $933 \mathrm{~Hz}$ und einem Strangstrom von $267 \mathrm{~A}$

Bei den in Abbildung 7-1 dargestellten $k_{r}$-Faktoren ist zu sehen, dass eine Simulation ohne Permanentmagnete für den betrachteten Betriebspunkt zu einer Reduktion der $k_{r}$-Faktoren führt. Dabei nehmen die Widerstandserhöhungsfaktoren insbesondere in den oberen Leiterlagen ab. Die Verläufe legen die Vermutung nahe, dass das fehlende Permanentmagnetfeld zu einem weniger starken Eindringen des Luftspaltfelds in die Nut führt. Dies wird durch die Längsfeldanteile bestätigt.

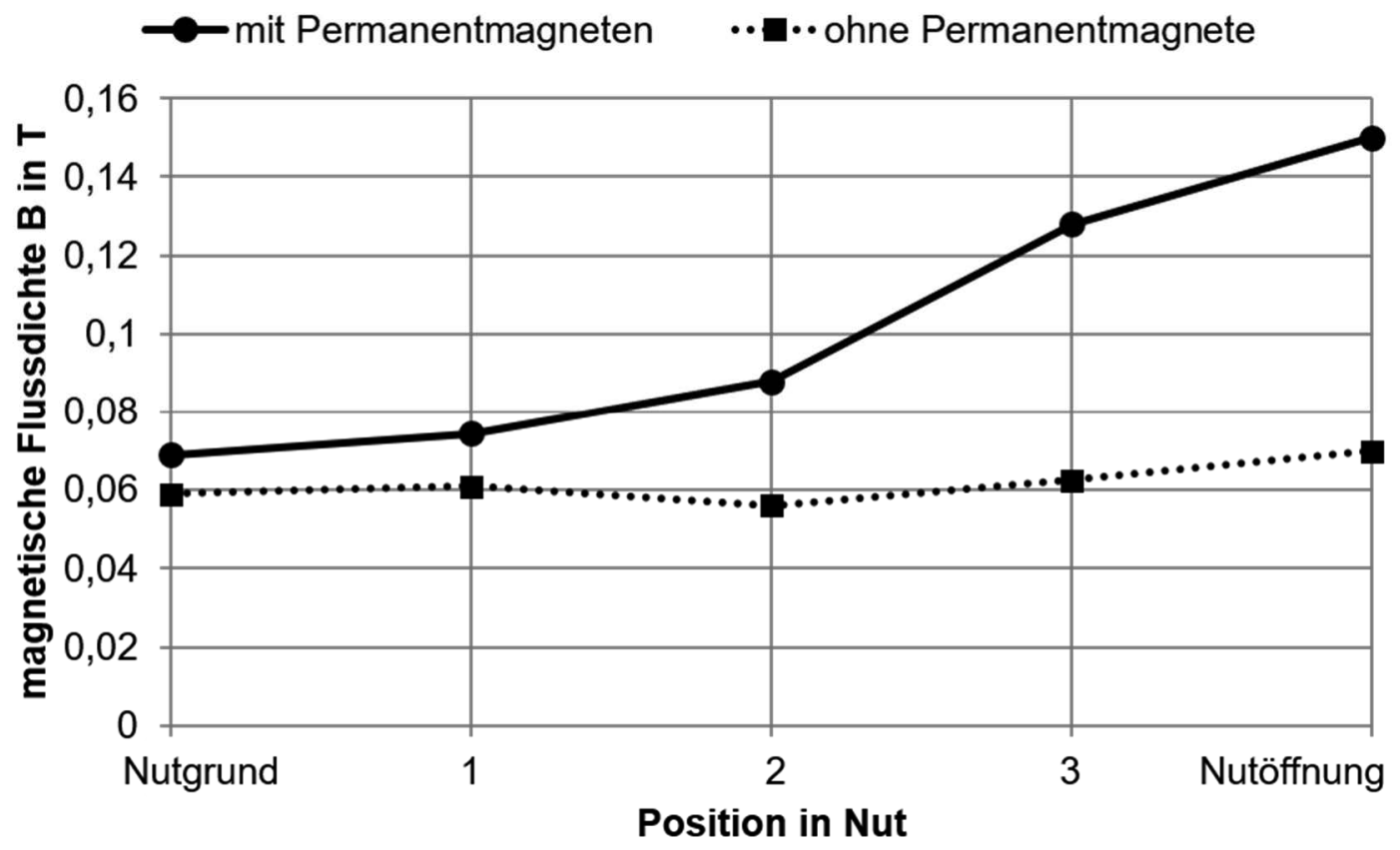

Abbildung 7-2: Magnetische Flussdichten in Längsrichtung mit und ohne Permanentmagnete (zur Erklärung der Darstellung siehe Abschnitt 4.2 und 4.3) 
Aus Abbildung 7-2 ist ersichtlich, dass die magnetische Flussdichte in Längsrichtung in der Nut durch das Fehlen der Permanentmagnete signifikant verringert wird. Auch hier ist die Abnahme an der Nutöffnung am größten und vermindert sich Richtung Nutgrund. Die Erklärung der verringerten Widerstandserhöhungsfaktoren ist somit einfach: Durch die fehlenden Permanentmagnete dringt kein zusätzliches magnetisches Feld mehr in die Nut ein, welches die Stromverdrängung in den oberen Leiterlagen verstärken könnte. Jedoch ist der Zusammenhang zwischen Luftspaltfeld und zusätzlicher Stromverdrängung nicht generell so einfach.

Für die Staffelung ergeben sich im Feldschwächbereich andere Zusammenhänge. In Abbildung 7-3 sind die $k_{r}$-Faktoren für drei Segmente dargestellt. Dabei ist neben dem Mittelsegment, das schon in Abbildung 7-2 enthalten ist, noch ein um $-3^{\circ}$ und ein um $+3^{\circ}$ gestaffeltes Segment aufgetragen.

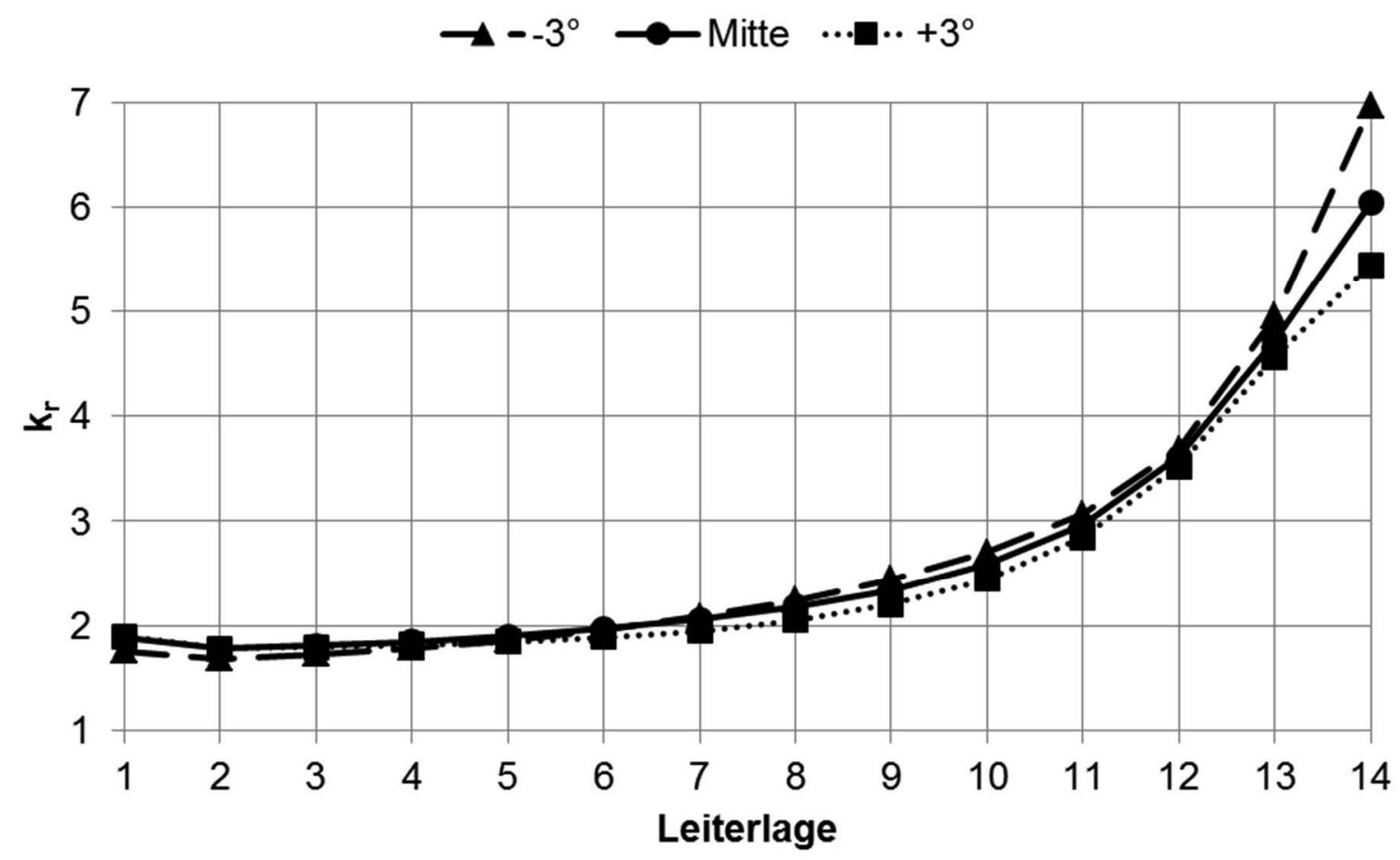

Abbildung 7-3: Gegenüberstellung von $k_{r}$-Verläufen in den Leitern 1 bis 14 (siehe Abbildung 4-3) für verschiedene axiale Abschnitte bei einer Frequenz von $933 \mathrm{~Hz}$ und einem Strangstrom von $267 \mathrm{~A}$

Es ist zu sehen, dass das negativ gestaffelte Segment in den beiden oberen Leiterlagen einen höheren $k_{r}$-Faktor aufweist, während sich der $k_{r}$-Faktor des positiv gestaffelten Segments in den oberen beiden Leiterlagen verringert. In der obersten Leiterlage beträgt die Differenz zwischen dem Widerstandserhöhungsfaktor des positiv und des negativ gestaffelten Segments immerhin 1,52. Auch hier liegt es nahe zu vermuten, dass das Luftspaltfeld aufgrund der Staffelung unterschiedlich stark in die Nut eindringt. Das ist jedoch nicht der Fall, wie aus Abbildung 7-4 hervorgeht. 


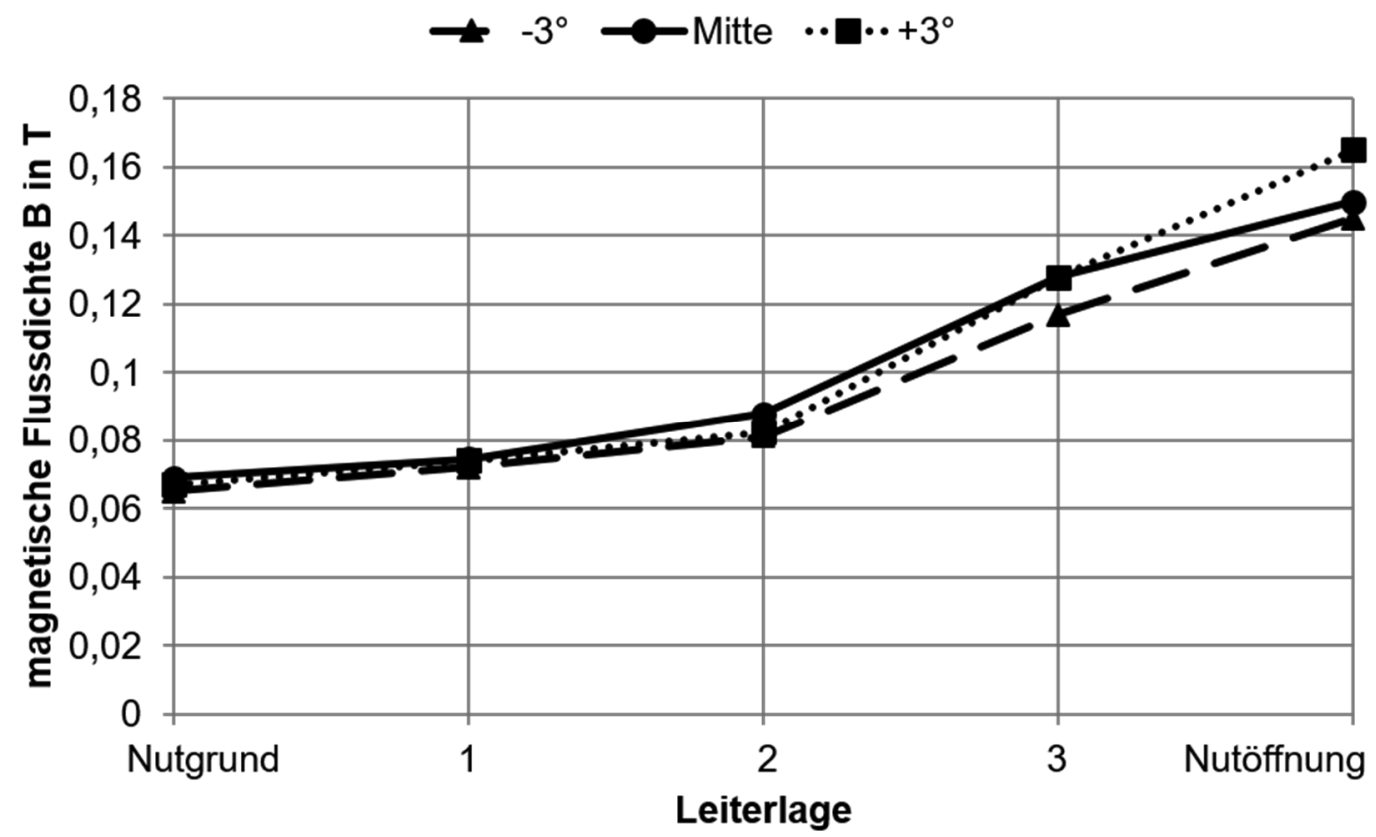

Abbildung 7-4: Magnetische Flussdichten in Längsrichtung für verschiedene Staffelungen des Rotors (zur Erklärung der Darstellung siehe Abschnitt 4.2 und 4.3)

In der Nut verändert sich die magnetische Flussdichte in Längsrichtung aufgrund der Schrägung kaum. Es ist sogar so, dass das um $+3^{\circ}$ gestaffelte Segment, welches im Bereich der Nutöffnung die höchste Flussdichte in Längsrichtung aufweist, in der obersten Leiterlage den geringsten Widerstandserhöhungsfaktor aufweist. Mit dem um $-3^{\circ}$ gestaffelten Segment verhält es sich genau umgekehrt.

Bei Betrachtung der anderen magnetischen Flussdichten in der Nut fällt auf, dass die magnetische Flussdichte in Querrichtung und die magnetische Flussdichte durch die Entlastung des Zahnkopfs mit der Stromverdrängung korrelieren. Beide sind in Abbildung 7-5 aufgetragen.

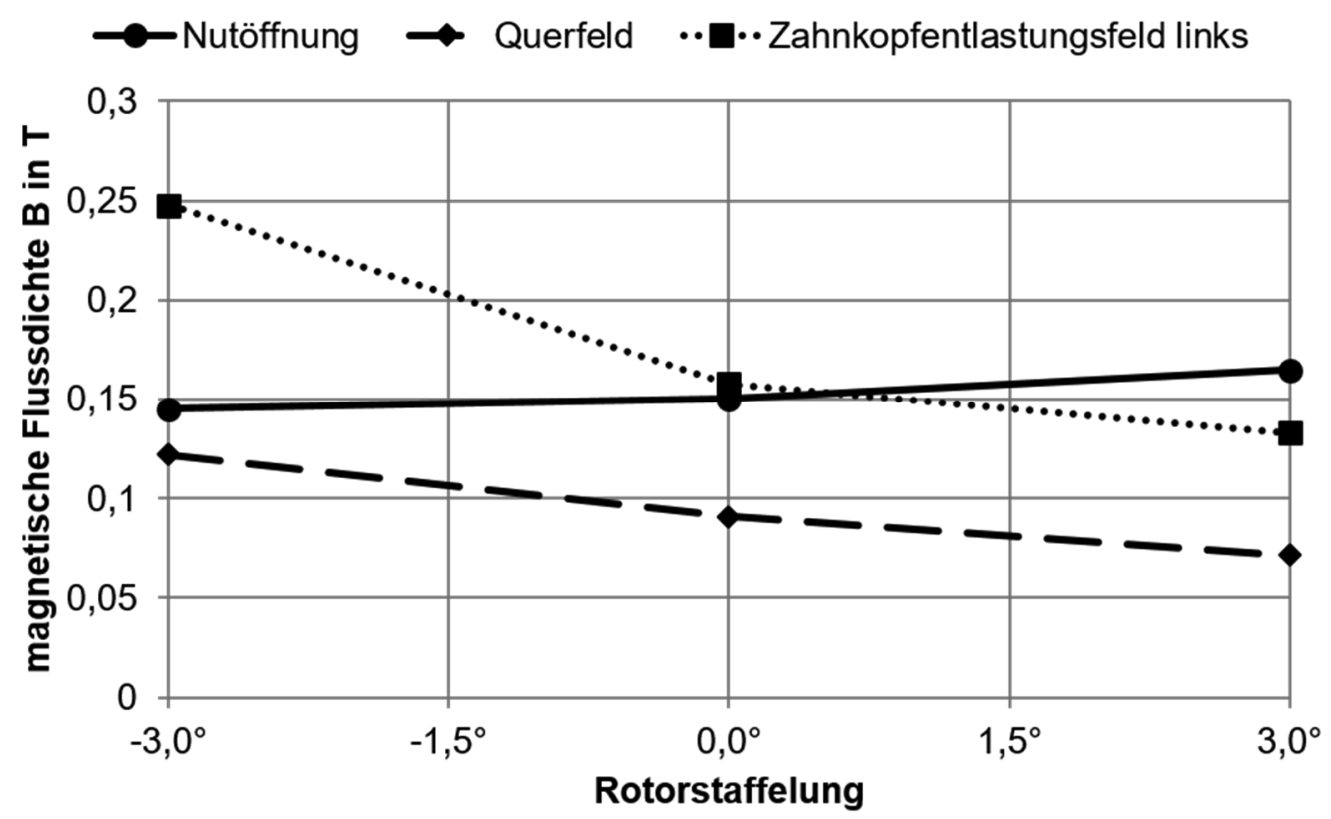

Abbildung 7-5: Einfluss der Rotorstaffelung auf die magnetischen Flussdichten in der Nut 
Die Darstellung zeigt unterschiedliche magnetische Flussdichten in der Nut und wie sie sich aufgrund der Rotorstaffelung verändern. Die Daten sprechen dafür, dass mit Zunahme der Rotorstaffelung in positiver Richtung das Permanentmagnetfeld vermehrt dem Statorfeld entgegenmagnetisiert. Dadurch reduziert sich das Luftspaltfeld. Dies vermindert auch das Querfeld und die magnetische Sättigung der Zahnköpfe. Beides bewirkt eine Verringerung der Widerstandserhöhungsfaktoren in den oberen Leiterlagen.

Darüber hinaus ist aus Abbildung 7-5 ersichtlich, dass die magnetische Flussdichte in Längsrichtung an der Nutöffnung entgegen dem Verhalten der Widerstandserhöhungsfaktoren mit zunehmend positiver Staffelung wächst.

Es scheint, als sei für die verschiedenen Widerstandserhöhungsfaktoren bei den unterschiedlichen Staffelungen nicht das in die Nut eindringende Hauptfeld ausschlaggebend. Vielmehr findet das für die Einhaltung der Spannungsgrenze notwendige entgegengesetzte Aufeinandertreffen von Rotor- und Statorfeld durch die Staffelung verschieden stark statt. Dies wirkt sich durch unterschiedliche magnetische Sättigungen des Eisenkreises im jeweiligen Segment auf die Widerstandserhöhungsfaktoren in den oberen Leiterlagen aus. Die Auswirkung der Staffelung auf die $k_{r}-$ Faktoren ist in Abbildung 7-6 für den Feldschwächbereich gezeigt.

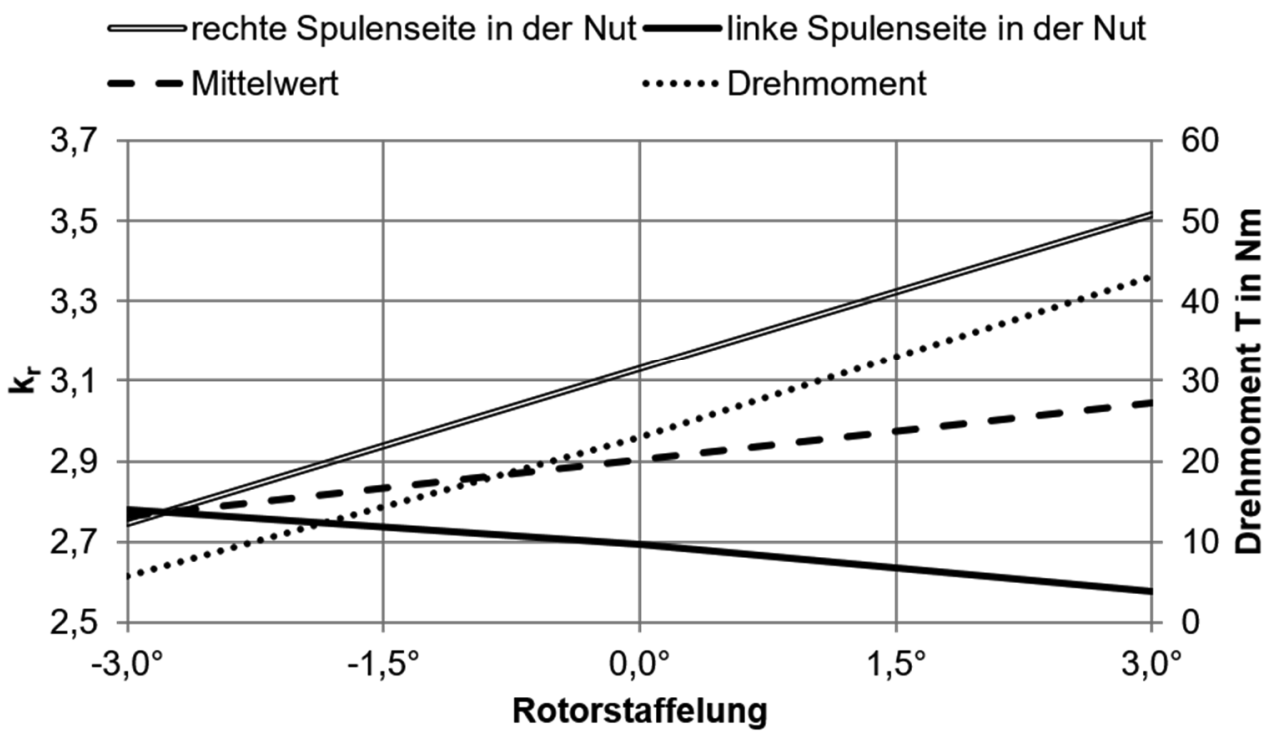

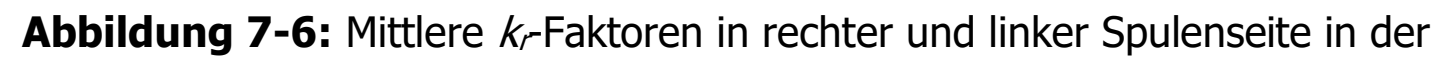
Nut in Abhängigkeit von der Staffelung. Zusätzlich ist der aus rechter und linker Spulenseite gemittelte mittlere $k_{r}$-Faktor der Nut angegeben sowie das erzeugte mittlere Drehmoment des jeweiligen Segments bei einer Frequenz von $933 \mathrm{~Hz}$ und einem Strangstrom von $267 \mathrm{~A}$

Aus der Abbildung geht hervor, dass der mittlere $k_{r}$-Faktor durch die Staffelung signifikant beeinflusst wird. Des Weiteren ergibt sich aus ihr, dass die beiden nebeneinander in der Nut liegenden Spulenseiten sehr unterschiedlich durch die Staffelung beeinflusst werden. Im $-3^{\circ}$ gestaffelten Segment liegt der mittlere Widerstandserhöhungsfaktor in den beiden nebeneinander in der Nut liegenden Spulenseiten noch fast gleichauf. Mit in positiver Richtung zunehmender Staffelung laufen die mittleren $k_{r}$-Faktoren auseinander. In der linken Spulenseite in der Nut nimmt der Widerstandserhöhungsfaktor $a b$, während er in der rechten zunimmt. Die Ursache für das unterschiedliche Verhalten ist, dass sich im rechten Nutbereich aufgrund des motori- 
schen Betriebs mehr Feldlinien ausbilden, wie es bei Gröninger u.a. [16] beschrieben wird. Das bedeutet, dass sich im rechten Nutbereich eine größere Querfeldkomponente ausbildet. Der aus beiden in der Nut liegenden Spulenseiten gemittelte mittlere $k_{r}$-Faktor nimmt in positiver Staffelungsrichtung zu. Zusätzlich zeigt die Abbildung 7-6 das erzeugte Drehmoment des jeweiligen Segments. Es ergibt sich die plausible Situation, dass in positiver Schrägungsrichtung sowohl die induzierte Spannung, als auch das Drehmoment und der mittlere Widerstandserhöhungsfaktor der Nut zunehmen. Das heißt, mit zunehmender Spannung und zunehmenden Wechselstromverlusten nimmt die abgegebene Leistung der elektrischen Maschine zu. Dies erscheint naheliegend.

Für den Feldschwächbereich hat sich ergeben, dass durch ein fehlendes Permanentmagnetfeld die $k_{r}$ Faktoren, insbesondere in den oberen Leiterlagen, reduziert werden. Das Permanentmagnetfeld verursacht bei der betrachteten elektrischen Maschine im Feldschwächbereich also zusätzliche Stromverdrängungsverluste. Allerdings ist der Fall nicht immer so einfach. So wurde anhand der Rotorstaffelung eine Situation dargestellt, in der das Permanentmagnetfeld die Stromverdrängungsverluste im Feldschwächbereich reduzieren kann. Jedoch geht diese Reduktion mit Drehmomentverlusten einher.

Zusammenfassend wurde gezeigt, dass die Position der Leiter in der Nut und die genaue Überlagerung von Permanentmagnet- und Statorfeld signifikanten Einfluss auf den Widerstandserhöhungsfaktor haben können. Dies analytisch zu erfassen, erscheint schwierig.

\subsection{Einfluss des Konstantflussbereichs und der Schrägung}

In diesem Abschnitt wird zunächst geklärt, warum es in Abbildung 3-11 trotz des Entfalls der Permanentmagnete zum Anstieg der $k_{r}$-Faktoren kommt. Denn ähnlich wie im Feldschwächbereich wird auch hier die Längsfeldkomponente im oberen Nutbereich signifikant reduziert, wie aus Abbildung 7-7 ersichtlich ist.

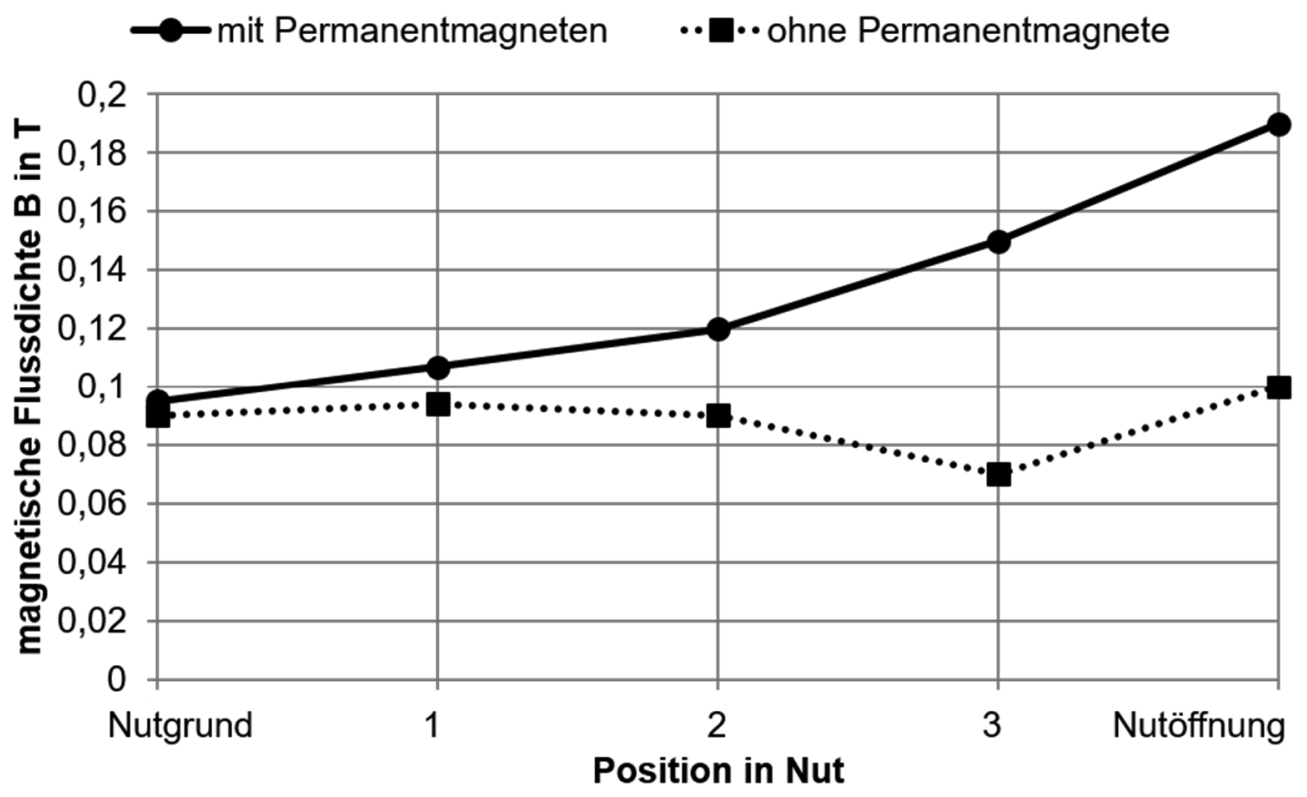

Abbildung 7-7: Magnetische Flussdichten in Längsrichtung mit und ohne Permanentmagnete (zur Erklärung der Darstellung siehe Abschnitt 4.2 und 
Der Unterschied ist jedoch, dass bei der betrachteten Maschine im Feldschwächbereich ein Betrieb an der Stromgrenze nicht sinnvoll ist. Im Konstantflussbereich wird die Maschine aber durchaus an der Stromgrenze betrieben. Das führt zu einer stärkeren magnetischen Sättigung des Eisenkreises. Dabei ist das Permanentmagnetfeld dem Statorfeld teilweise entgegengerichtet, was die Sättigung des Eisenkreises reduziert. Durch den Entfall der Permanentmagnete steigt die Sättigung und in der Folge auch das Zahnentlastungsfeld an, wie aus Abbildung 7-8 hervorgeht.

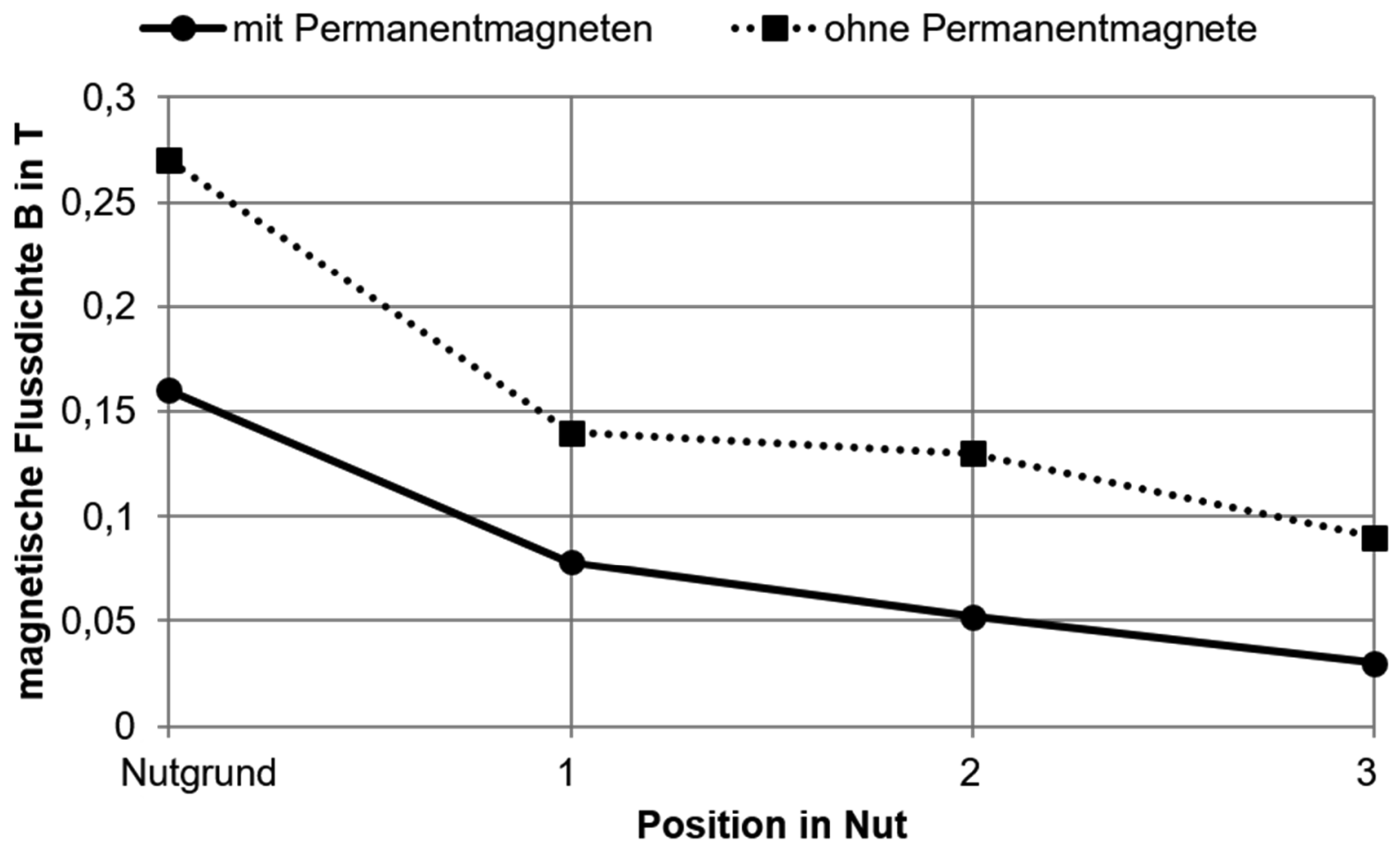

Abbildung 7-8: Anstieg des Zahnentlastungsfelds (siehe Abbildung 5-2) durch den Entfall der Permanentmagneten bei einer Frequenz von $467 \mathrm{~Hz}$ und einem Strangstrom von $419 \mathrm{~A}$

Dadurch steigen die Widerstandserhöhungsfaktoren im unteren und im mittleren Nutbereich an. Ähnlich verhält es sich im Konstantflussbereich mit der Staffelung. Abbildung 7-9 zeigt die $k_{r}$-Faktoren für drei unterschiedlich geschrägte Segmente. 


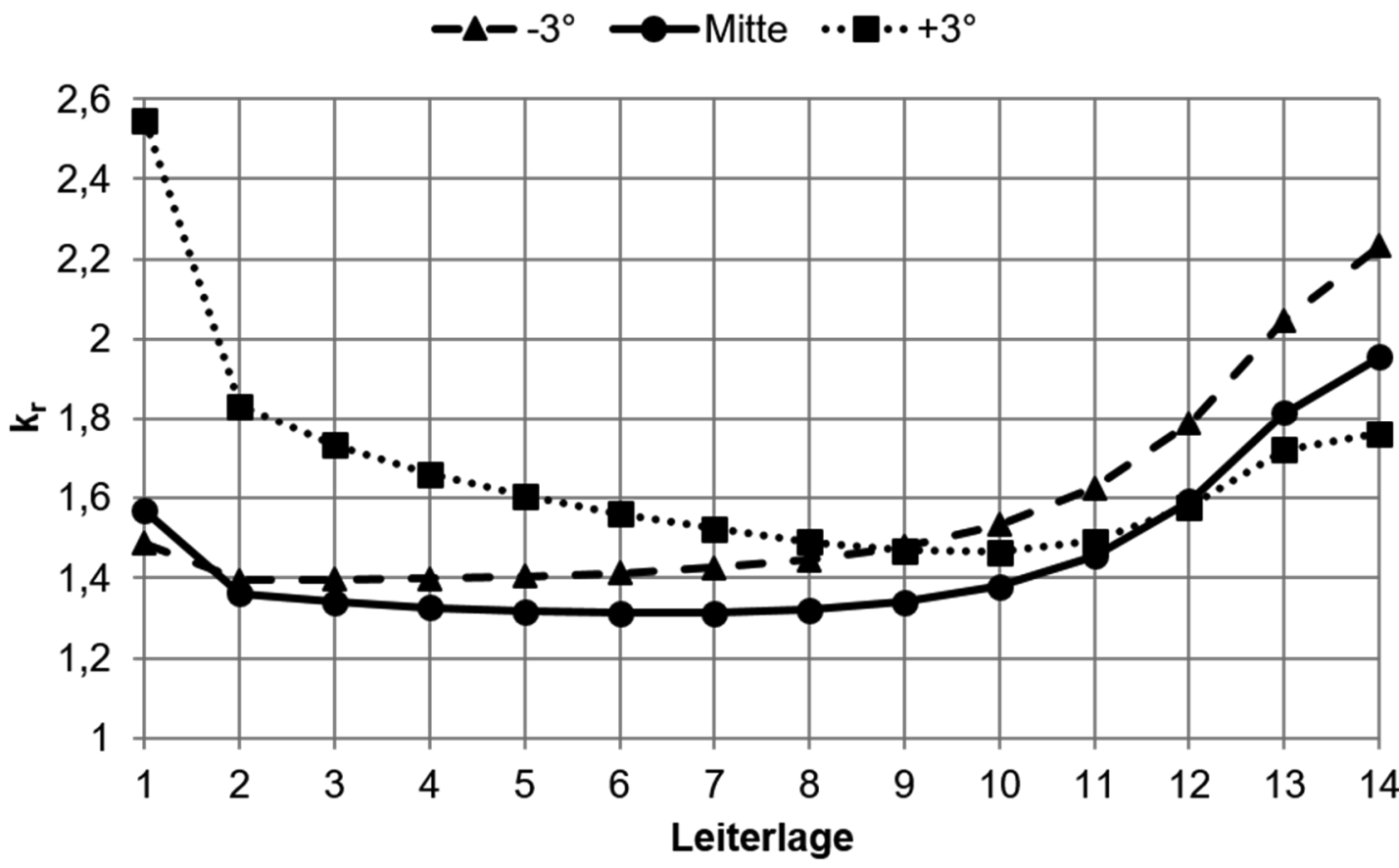

Abbildung 7-9: $k_{r}$-Verläufe in den Leitern 1 bis 14 (siehe Abbildung 4-3) in der links in der Nut liegenden Spulenseite für verschiedene Staffelungen bei einer Frequenz von $467 \mathrm{~Hz}$ und einem Strangstrom von $419 \mathrm{~A}$

Dabei ist gut zu erkennen, dass das um $-3^{\circ}$ gestaffelte Segment im oberen Nutbereich die höchsten Widerstandserhöhungsfaktoren erreicht. Bei den anderen Segmenten nehmen die $k_{r}$-Faktoren in den oberen Leiterlagen ab, während sie in den unteren Leiterlagen teilweise deutlich steigen. Dies ist auf die wachsende magnetische Sättigung des Eisenkreises zurückzuführen, wie der Abbildung 7-10 zu entnehmen ist.

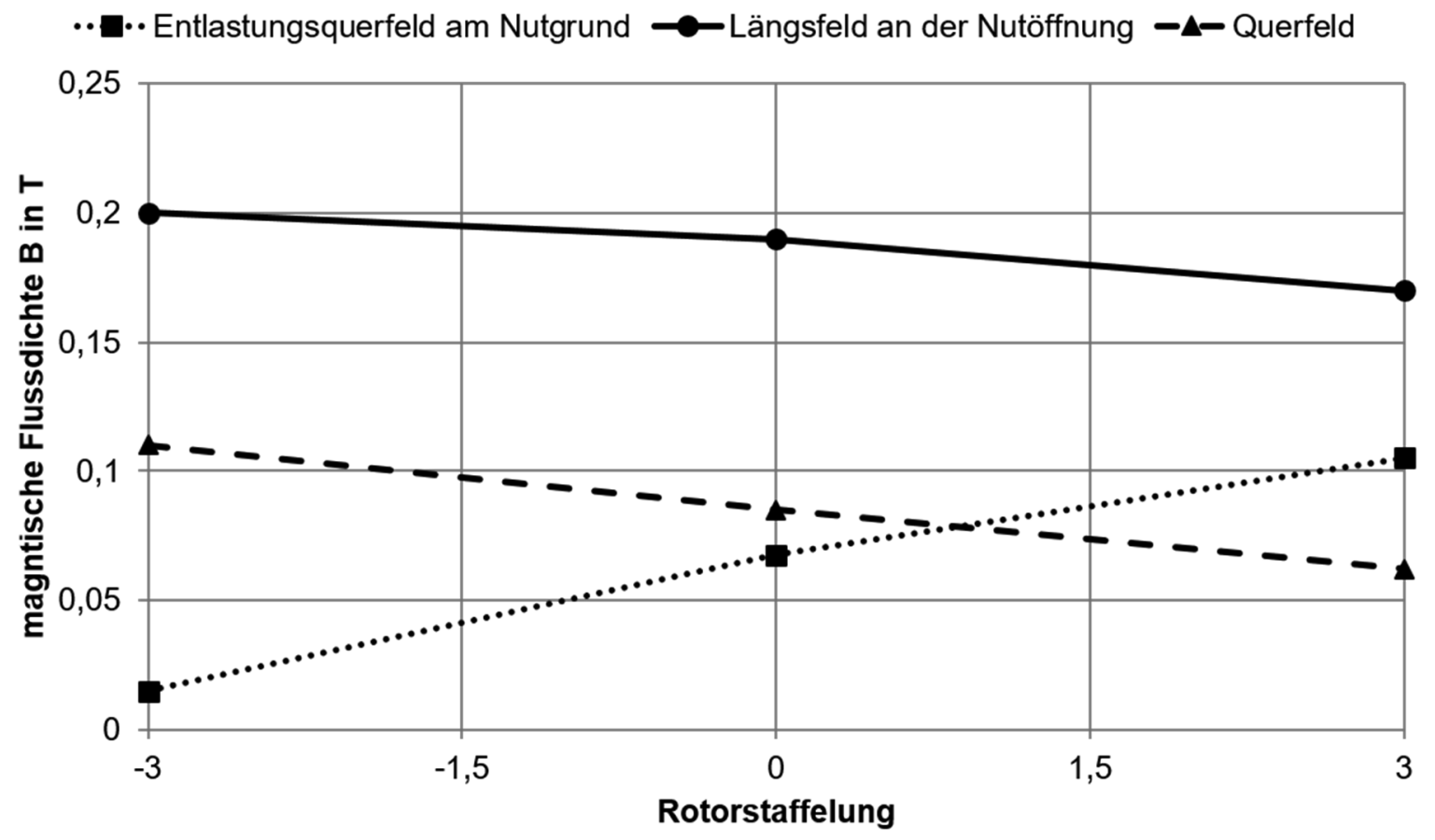

Abbildung 7-10: Einfluss der Rotorstaffelung auf die magnetischen Flussdichten in der Nut 
Sie zeigt, dass sich das Querfeld und das in die Nut eindringende Luftspaltfeld mit in positiver Richtung zunehmender Staffelung verringern. Beide sind für Stromverdrängung in den oberen Leiterlagen verantwortlich. Gleichzeitig nimmt das Entlastungsfeld am Nutgrund zu. Die Verläufe deuten an, dass mit steigender Staffelung in positiver Richtung die Permanentmagnete immer weniger dem Statorfeld entgegenmagnetisieren. Dadurch wird der Eisenkreis stärker in die magnetische Sättigung getrieben. Dies erklärt das Sinken des Querfelds bei gleichzeitigem Anstieg des Entlastungsfelds.

Die Beeinflussung des Entlastungsfelds durch die Rotorstaffelung wird auch noch einmal aus Abbildung 7-11 deutlich.

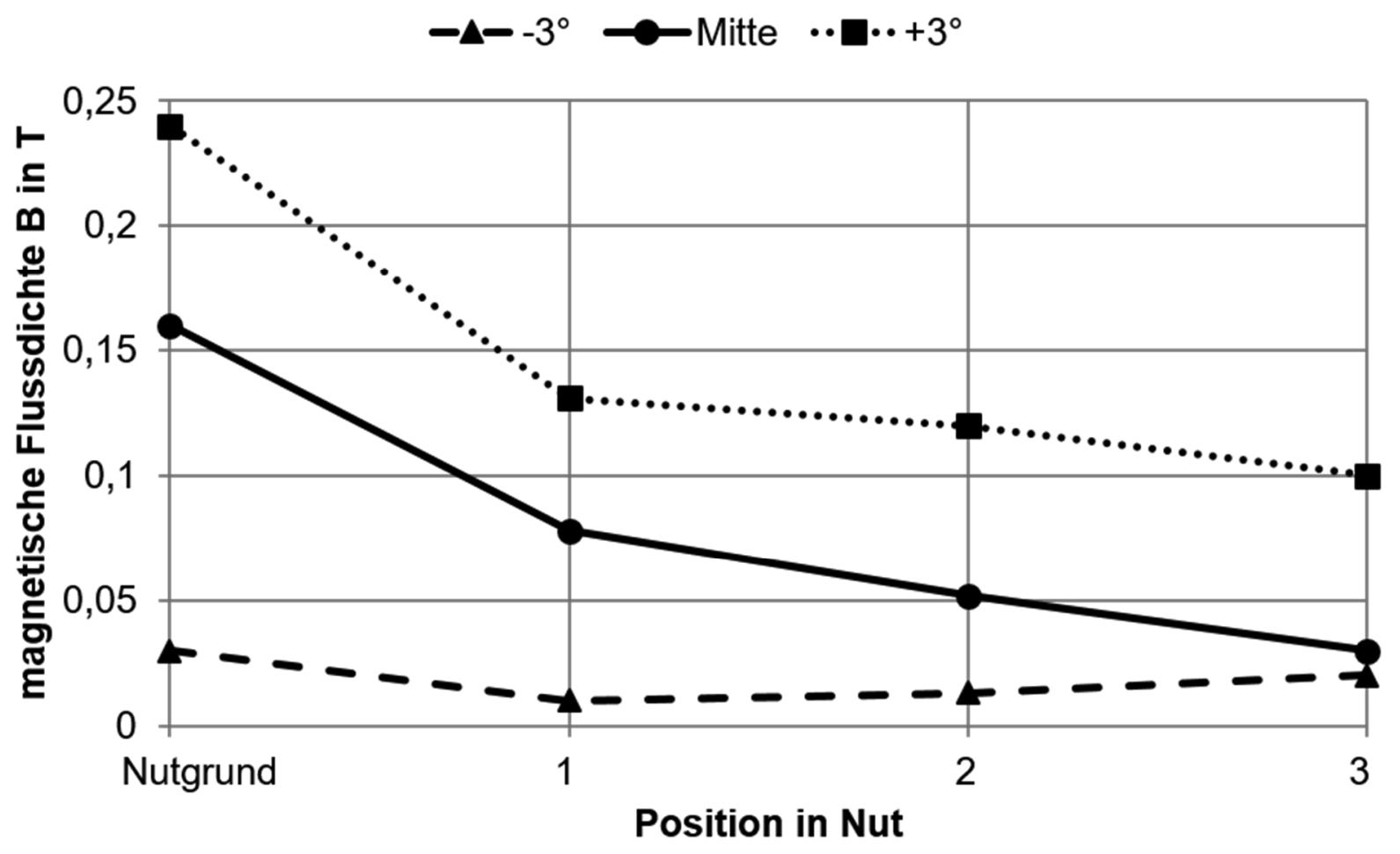

Abbildung 7-11: Einfluss der Staffelung auf das Zahnentlastungsfeld (siehe Abbildung 5-2) bei einer Frequenz von $467 \mathrm{~Hz}$ und einem Strangstrom von $419 \mathrm{~A}$

Hier ist die magnetische Flussdichte in Längsrichtung in der Nut für die verschiedenen Staffelungen dargestellt. Mit zunehmend positiver Staffelung nimmt sie insbesondere im unteren Nutbereich zu.

Aus dem gegenläufigen Verhalten von Zahnentlastungs- und Querfeld in Folge der Rotorstaffelung ergibt sich eine interessante Konsequenz. Denn entsprechend den Feldern verhält sich auch die Stromverdrängung. Die $k_{r}$-Faktoren in den oberen Lagen sinken, während sie in den unteren Leiterlagen ansteigen. Dadurch ergibt sich für den mittleren $k_{\digamma}$-Faktor in der links in der Nut liegenden Spulenhälfte ein lokales Minimum, welches der Abbildung 7-12 zu entnehmen ist. 


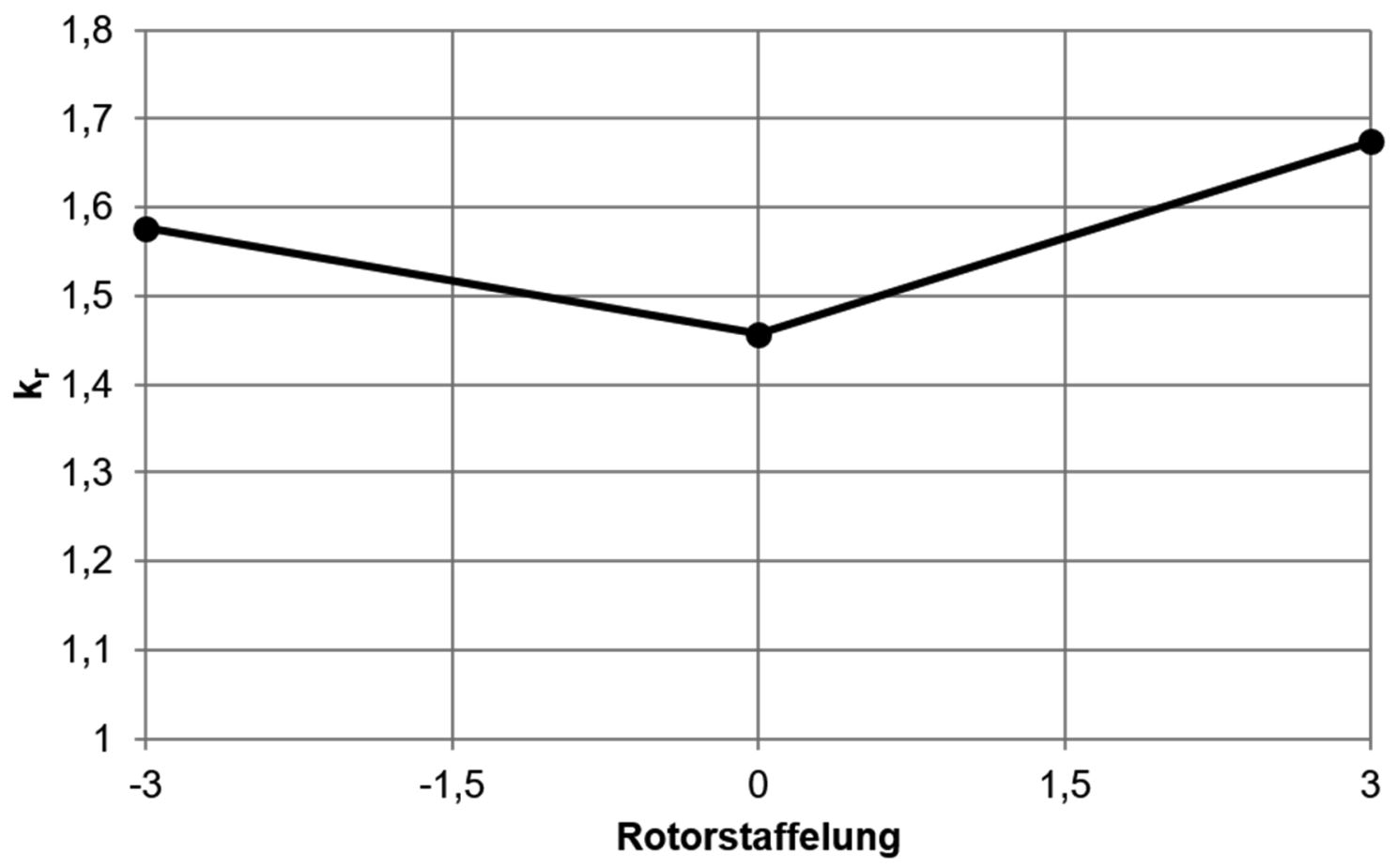

Abbildung 7-12: Mittlerer $k_{r}$-Faktor der links in der Nut liegenden Spulenhälfte für verschiedene Rotorstaffelungen bei einer Frequenz von $467 \mathrm{~Hz}$ und einem Strangstrom von 419 A

Betrachtet auf die gesamte Nut sind die Verhältnisse jedoch andere, da sich linke und rechte Spulenhälfte in der Nut wieder unterschiedlich verhalten. Dies lässt sich exemplarisch anhand von Abbildung 7-13 erkennen.

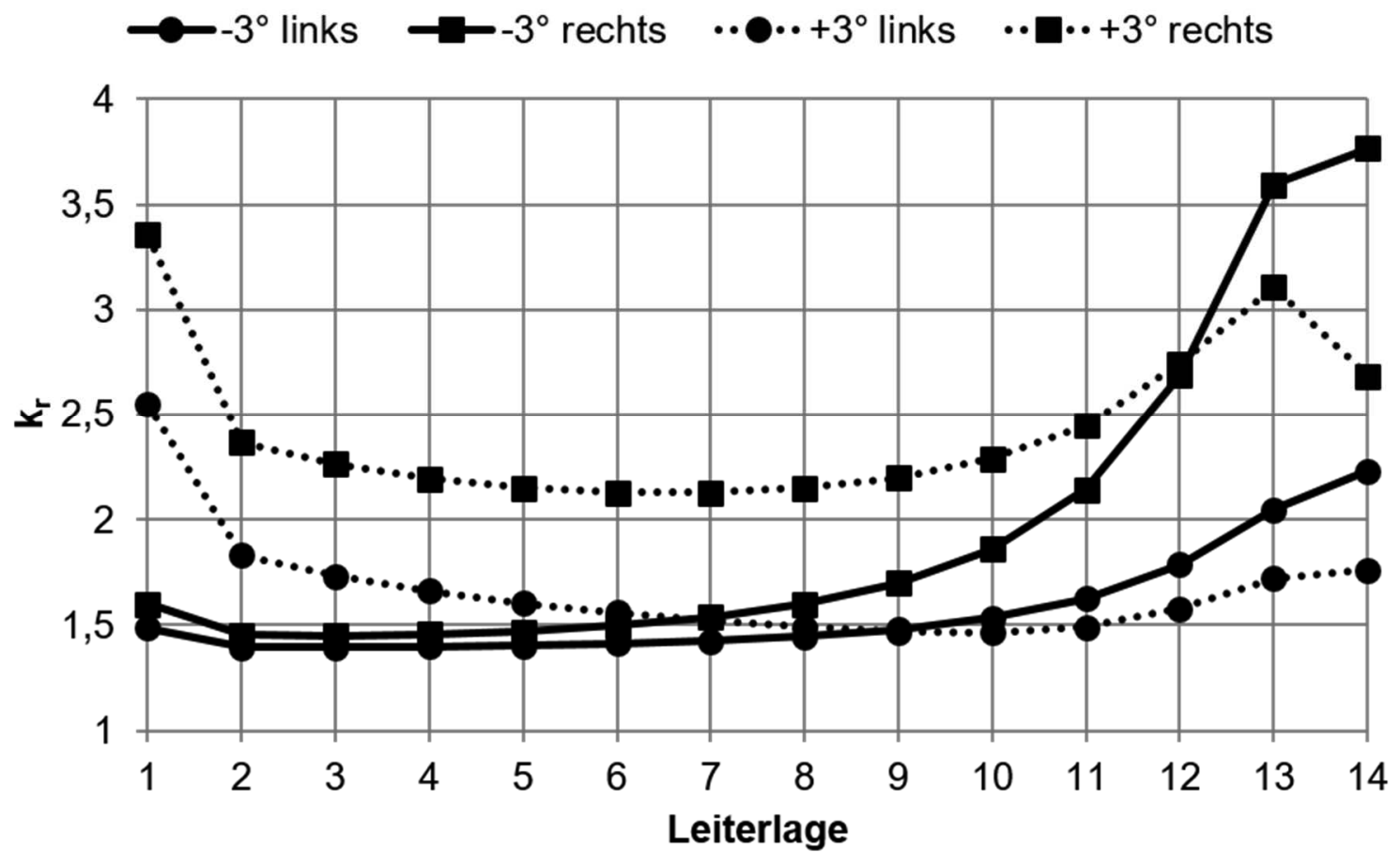

Abbildung 7-13: Gegenüberstellung von $k_{r}$-Verläufen in den Leitern 1 bis 14 (siehe Abbildung 4-3) für verschiedene Staffelungen und in der linken und rechten in der Nut liegenden Spulenhälfte bei einer Frequenz von $467 \mathrm{~Hz}$ und einem Strangstrom von 419 A 
Wie im Feldschwächbereich ist auch im Konstantflussbereich das Querfeld in der rechten Nuthälfte stärker ausgeprägt. In der Folge liegt der Widerstandserhöhungsfaktor in den oberen Lagen der rechten Spulenhälfte höher als in der linken Spulenhälfte. Mit zunehmender Staffelung in positiver Richtung ist die rechte Spulenhälfte auch stärker von Zahnentlastungsfeldern betroffen. Dies führt zu höheren $k_{r}$ Faktoren im unteren Nutbereich.

Betrachtet man nun den Verlauf der mittleren $k_{r}$-Faktoren über die Rotorstaffelung

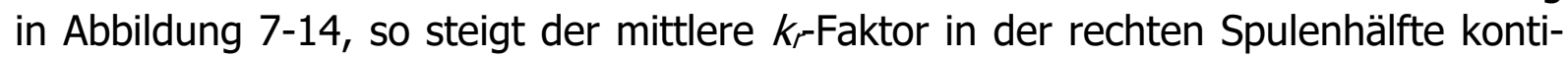
nuierlich an.

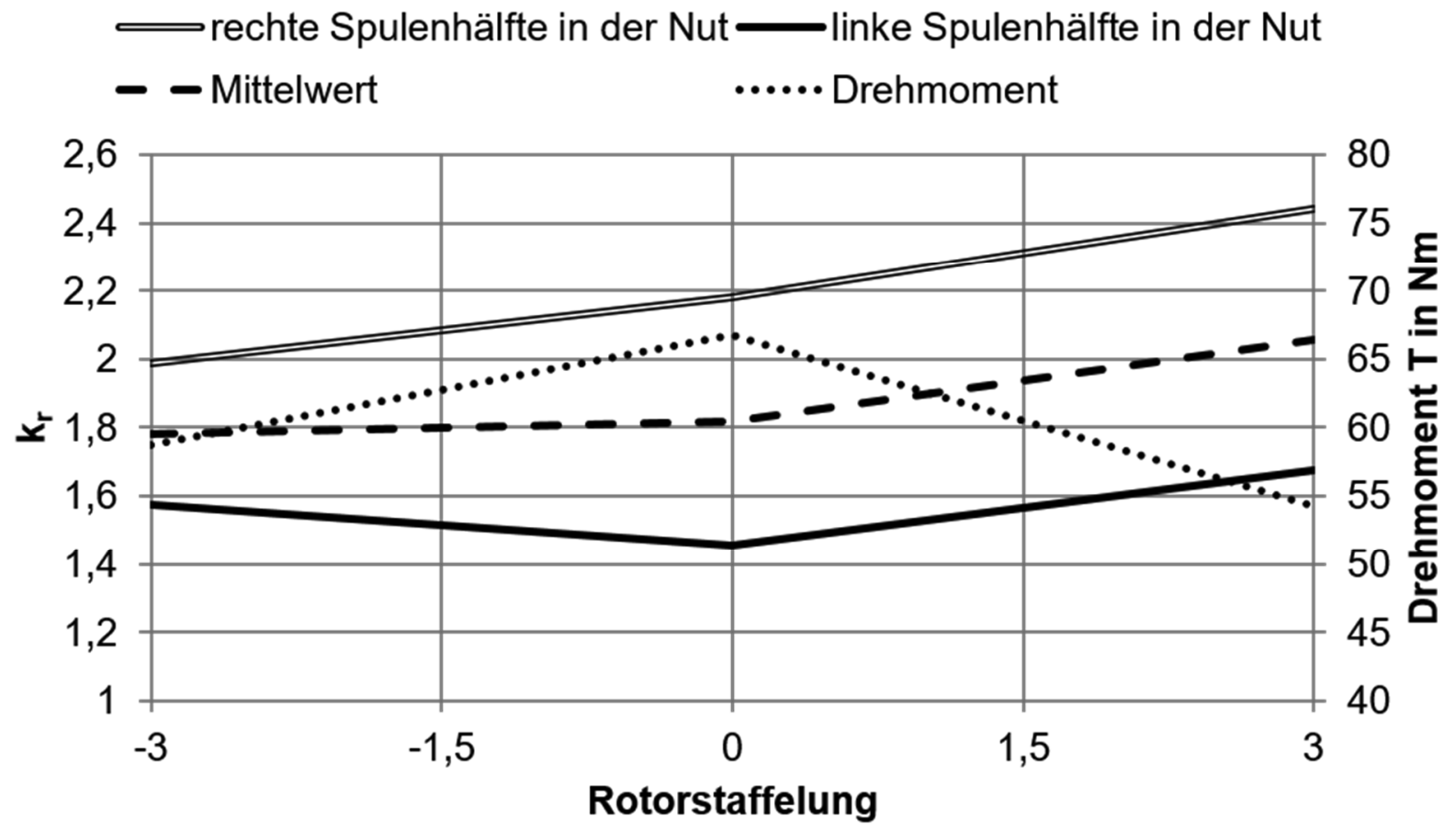

Abbildung 7-14: Mittlere $k_{r}$-Faktoren in rechter und linker Spulenhälfte in der Nut in Abhängigkeit von der Staffelung. Zusätzlich ist der mittlere $k_{r}$-Faktor der Nut angegeben sowie das erzeugte mittlere Drehmoment des jeweiligen Segments bei einer Frequenz von $467 \mathrm{~Hz}$ und einem Strangstrom von $419 \mathrm{~A}$

Im Gegensatz dazu fällt der mittlere $k_{r}$-Faktor in der linken Spulenhälfte vom $-3^{\circ}{ }_{-}$ Segment zum Mittelsegment hin zunächst ab. Anschließend steigt er etwas stärker zum $+3^{\circ}$-Segment hin an. Bezogen auf die gesamte Nut bleibt der mittlere $k_{r}$ Faktor vom $-3^{\circ}$-Segment bis zum Mittelsegment hin nahezu konstant und steigt Richtung $+3^{\circ}$-Segment an. Interessant ist nun das Verhalten des Drehmoments. Dieses hat sein Maximum im Mittelsegment. Von diesem aus betrachtet, fällt das Drehmoment Richtung positiver Staffelung stärker $a b$, während der mittlere Widerstandserhöhungsfaktor gleichzeitig ansteigt. In Richtung negativer Staffelung fällt das Drehmoment weniger stark $a b$, während der mittlere Widerstandserhöhungsfaktor annähernd konstant bleibt. Eine tendenzielle Ausrichtung der Rotormitte in negativ gestaffelter Richtung bedeutet dann geringere Kupferverluste bei gleichzeitiger Steigerung des Drehmoments. Andere Verlustarten sind bei dieser Betrachtung nicht betrachtet worden.

Das gezeigte Verhalten gibt einen Eindruck, wie schwer es ist, einfache analytische Zusammenhänge für die Interaktion von Rotor- und Statorfeld zu ermitteln, insbe- 
sondere wenn diese allgemeingültig sein sollen. Ebenso wird aufgezeigt, dass es durch die Regelung der Maschine möglich ist, die Stromverdrängungsverluste geringfügig zu reduzieren, ohne Einbußen beim Drehmoment zu erleiden.

\subsection{Drehmomentbildung und Zusammenfassung}

Auch die Drehmomentbildung aus synchronem Moment und Reluktanzmoment beeinflusst, wie sich Rotor- und Statorfeld überlagern. Betrachtet man Rotoren mit eingebetteten Magneten, so haben bereits die Geometrie und die Anzahl der Magnete und die Magnettaschen Einfluss auf die Drehmomentbildung. Auch die Remanenzflussdichte $B_{r}$ der Magnete beeinflusst das Ergebnis. Zur Erhöhung des Reluktanzmoments werden häufig noch zusätzliche Flusssperren und Kavitäten eingebracht. Dies wird auch getan, um die Massenträgheit zu beeinflussen. All diese Aspekte analytisch zu erfassen, insbesondere in Kombination mit einem Eisenkreis, der im Betrieb durchaus in den Sättigungsbereich gebracht wird und dadurch zusätzlich die Gesetzmäßigkeiten der Stromverdrängung beeinflusst, mutet nahezu unmöglich an.

Die Betrachtungen in diesem Kapitel zeigen, dass für eine optimale Gestaltung und Regelung der elektrischen Maschine die Stromverdrängungsverluste berücksichtigt werden müssten. Gleichzeitig ergeben sich durch das Zusammenspiel von Rotor- und Statorfeld komplexe Zusammenhänge für die zusätzliche Stromverdrängung in den oberen Leiterlagen gemäß Abbildung 6-11, die sich auf analytischem Wege nicht oder nur schwer bestimmen lassen. Dies erschwert es wiederum, die Gesamtauswirkungen der Stromverdrängung im Auslegungsprozess vollständig zu berücksichtigen. Hier ist es erforderlich, sich auf die größten Einflüsse zu beschränken.

Für das in die Nut eindringende Luftspaltfeld wird hier, aufgrund der aufgezeigten Einflussvielfalt, keine quantitative Bestimmung der Stromverdrängung vorgenommen. Es wird auf die im Stand der Technik bekannten und in Abschnitt 1.3 benannten qualitativen Regeln für den Auslegungsprozess verwiesen. 


\section{Schlussbetrachtung}

In der vorliegenden Arbeit wird Stromverdrängung anhand einer modernen, hoch ausgenutzten, umrichterbetriebenen Synchronmaschine mit eingebetteten Permanentmagneten zur Nutzung des Reluktanzmoments und Zahnspulen zur Verringerung der Wickelkopflänge untersucht. Es zeigt sich, dass der in Abschnitt 2.1.4 beschriebene, über hundert Jahre bewährte, analytische Berechnungsansatz zur Bestimmung der Stromverdrängung bei dieser elektrischen Maschine keine zuverlässigen Ergebnisse liefert. Begünstigt wird dies dadurch, dass die Spulenquerschnittsgeometrie der betrachteten elektrischen Maschine nach den Erkenntnissen des bewährten analytischen Verfahrens gestaltet ist. Die verwendeten gegossenen Spulen sind breit und flach ausgeführt, um eine geringe Anfälligkeit für Nutquerfelder aufzuweisen. Dadurch sind sie anfällig für Längsfelder, da sie für diese eine große Angriffsfläche bieten. Durch die breiten Leiter erhalten zusätzliche, längsfeldverursachende Effekte einen signifikanten Einfluss. Diese müssen bei breiten Leitern für eine ausreichend genaue Vorhersage der Stromverdrängung berücksichtigt werden.

Um die zusätzlichen Stromverdrängungsursachen zu bestimmen, werden in weiten Teilen Untersuchungen mittels Finite-Elemente-Rechnungen durchgeführt, da sich auf diese Weise recht schnell ein Verständnis der Verhältnisse erreichen lässt. Dazu muss zunächst eine geeignete Form für die Auswertung der Finite-ElementeSimulationen entwickelt werden, denn für Stromverdrängung gibt es dazu bisher noch kein etabliertes Vorgehen. Es erweist sich als zielführend, zum einen den Widerstandserhöhungsfaktor in jedem Leiter einzeln zu betrachten und zum anderen auch die Ausbildung des magnetischen Felds in der Nut zu berücksichtigen. Dadurch wird ersichtlich, welche Faktoren in welchem Ausmaß das magnetische Feld in der Nut und in der Folge auch die Stromverdrängung beeinflussen. Anschließend werden die Beziehungen nach Möglichkeit analytisch formuliert. Bei diesem Vorgehen werden drei wesentliche Einflussfaktoren auf die Stromverdrängung identifiziert und studiert: die magnetische Sättigung des Eisenkreises, die Spulenanordnung in der Nut und die Wechselwirkung mit dem Luftspaltfeld.

Zuerst wird in Kapitel 5 die Auswirkung der magnetischen Sättigung des Eisenkreises auf das Nutfeld und die Stromverdrängung untersucht. Dabei werden zwei wesentliche Beeinflussungen ausgemacht. Zum einen ist dies eine Verminderung der Stromverdrängung durch das Querfeld, da dieses nicht mehr im gleichen Maße mit dem Strom ansteigt wie im nicht gesättigten Bereich. Die Verminderung reduziert die zusätzlichen Verluste insbesondere in den oberen Leiterlagen. Die zweite wesentliche Beeinflussung sind die auftretenden magnetischen Längsfelder, die sich in der Nut zur Entlastung der gesättigten Eisenstrecken ausbilden. Hierbei wird insbesondere die Sättigung der Zähne tiefergehend beleuchtet. Es wird gezeigt, dass sich die magnetische Sättigung in Abhängigkeit von der Leiterquerschnittsgeometrie sowohl steigernd als auch reduzierend auf die Zusatzverluste durch Stromverdrängung auswirken kann. Die beiden Auswirkungen der magnetischen Sättigung werden anschließend analytisch beschrieben. Dabei kann für die Verminderung des Querfelds auf bestehenden Ansätzen aufgebaut werden, während für die Berücksichtigung des Zahnentlastungsfelds ein neuer Ansatz entwickelt wird. Hier besteht sicherlich noch Potenzial zur weiteren Verfeinerung der analytischen Methoden. Jedoch können 
erstmals die grundsätzlichen Wirkzusammenhänge in diesem Umfang aufgezeigt werden.

In Kapitel 6 wird die nebeneinanderliegende Spulenseitenanordnung in einer Nut behandelt, wie sie typischerweise bei Zahnspulen vorkommt. Auch hier kann an den Wissensstand angeknüpft werden. Mosebach [29] sagt für nebeneinanderliegende Spulenseiten in einer Nut mit zunehmender Phasenverschiebung der Spulenströme eine Verringerung der Stromverdrängung vorher. Ähnlich wie für die magnetische Sättigung des Eisenkreises wird gezeigt, dass neben der Verminderung der Querfeldkomponente auch hier eine Längsfeldkomponente entsteht. Diese ist bei breiten Leitern in der Lage, die zusätzlichen Stromverdrängungsverluste signifikant zu erhöhen, insbesondere in den unteren Leiterlagen. Auch für die nebeneinanderliegende Anordnung von zwei Spulenseiten in einer Nut wird ein analytischer Berechnungsansatz zur Bestimmung der entstehenden Stromverdrängungsverluste entwickelt.

Zuletzt wird in Kapitel 7 die Wechselwirkung mit dem Luftspaltfeld als Ursache der Stromverdrängung untersucht. Hier findet sich im Stand der Technik eine Vielzahl von Veröffentlichungen, die sich des Themas annehmen. Jedoch wird dabei in der Regel nur das Eindringen des Luftspaltfelds in die Nuten berücksichtigt. Der Beitrag der vorliegenden Arbeit zu dem Thema ist eine Betrachtung der Faktoren, die die Bildung des Luftspaltfelds aus Stator- und Rotorbeitrag beeinflussen und wie sich dies auf die Stromverdrängung auswirkt. Hierbei wird erstmals auch die Beeinflussung der magnetischen Sättigung des Eisenkreises durch das Permanentmagnetfeld des Rotors betrachtet. Es wird aufgezeigt, dass es durch das Zusammenspiel von Staffelung, magnetischer Sättigung und Umrichterbetrieb sowie durch die Gestaltung des Rotors zur Nutzung des Reluktanzmoments zu signifikanten Beeinflussungen der Stromverdrängung durch das Luftspaltfeld kommt. Es wird festgestellt, dass dies den gesamten Nutbereich betreffen kann und nicht nur die Stromverdrängung in den oberen Leiterlagen. Aufgrund der vielen Einflüsse und Zusammenhänge ist eine analytische Vorhersage der durch die Wechselwirkung mit dem Luftspaltfeld entstehenden Stromverdrängung äußerst schwierig. Daher wird in dieser Arbeit auf einen analytischen Ansatz zur Bestimmung der Stromverdrängung durch die Wechselwirkung mit dem Luftspaltfeld verzichtet und stattdessen auf die qualitativen Empfehlungen aus dem Stand der Technik verwiesen.

Die vorliegende Arbeit zeigt, dass das etablierte, über hundert Jahre alte, analytische Berechnungsverfahren mit seiner Annahme eines reinen Nutquerfelds für hoch ausgenutzte elektrische Maschinen nicht mehr uneingeschränkt zulässig ist. Daher münden die durchgeführten Betrachtungen zum Teil in Ergänzungen des bewährten, analytischen Modells.

Eine Überprüfung der in dieser Arbeit entwickelten analytischen Berechnungsansätze an anderen elektrischen Maschinen wäre interessant, um die Übertragbarkeit der Ansätze sicherzustellen. Des Weiteren wäre eine Verallgemeinerung des Begriffs der kritischen Leiterhöhe durch eine kritische Leitergeometrie wünschenswert. Die gewonnen Erkenntnisse deuten an, dass es neben der kritischen Leiterhöhe auch eine kritische Leiterbreite gibt. Das würde bedeuten, dass betriebspunktabhängig unterschiedliche Leitergeometrien und -querschnitte ideal wären. Die kritische Leitergeometrie würde eine Optimierung auf bestimmte Arbeitspunkte hin unterstützen. 


\section{Literaturverzeichnis}

[1] Backhaus, U., Energietransport durch elektrische Ströme und elektromagnetische Felder. Essen, Magdeburg: Westarp, 1993.

[2] Bickel, B., Hübner, M. und Franke, J., "Analyse des Optimierungspotenzials zur Erhöhung des Kupferfüllfaktors in elektrischen Maschinen," ant Journal, Nr. 53 (2), S. 16-21, 2014.

[3] Binder, A., Elektrische Maschinen und Antriebe: Grundlagen, Betriebsverhalten. SpringerLink: Bücher. Berlin, Heidelberg: Springer, 2012.

[4] Böhme, J. F., Stochastische Signale eine Einführung in Modelle, Systemtheorie und Statistik. Teubner-Studienbücher: Elektrotechnik. Stuttgart: Teubner, 1993.

[5] Chari, M. und Csendes, Z. J., "Finite element analysis of the skin effect in current carrying conductors," Magnetics, IEEE Transactions on, Nr. 5, S. 1125-1127, 1977.

[6] Dabrowski, M. und Demenko, A., "Hybrid Method For Analysis Of Eddy-Current Loss In Electrical Machine Winding Caused By The Main Flux," Magnetics, IEEE Transactions on, Nr. 1, S. 479-482, 1988.

[7] Dwight, H. B., Electrical Coils and conductors: Their electrical characteristics and theory, 1.ed. 2.impr. New York usw: McGraw-Hill, 1945.

[8] Emde, F., "Einseitige Stromverdrängung in Ankernuten," Elektrotechnik und Maschinenbau, S. 703, 1908.

[9] Emde, F., "Über einseitige Stromverdrängung," Elektrotechnik und Maschinenbau, Nr. 26, S. 301-304, 1922.

[10] Endert, F., Heidrich, T., Schwalbe, U., Szalai, T. und Ivanov, S. D., "Effects of current displacement in a PMSM traction drive with single turn coils," 2013 International Electric Machines \& Drives Conference (IEMDC 2013): Chicago, Illinois, USA, 12 - 15 May 2013, S. 160-165, 2013.

[11] Field, A. B., "Eddy Currents in Large Slot-Wound Conductors," American Institute of Electrical Engineers, Transactions of the, vol. 24, S. 761-788, 1905.

[12] Fischer, R., Elektrische Maschinen, 15., aktualisierte Aufl. München [u.a.]: Hanser, 2011.

[13] Föppl, A., Einführung in die maxwellsche Theorie der Elektrizität, 5. Aufl. Theorie der Elektrizität. Leipzig Berlin: B.G.Teubner, 1918.

[14] Gerling, D., Electrical machines: Mathematical fundamentals of machine topologies. Mathematical Engineering.

[15] Gilman, R. E., "Eddy Current Losses in Armature Conductors," American Institute of Electrical Engineers, Transactions of the, vol. XXXIX, Nr. 1, S. 997-1056, 1920.

[16] Groninger, M., Horch, F., Kock, A., Jakob, M. und Ponick, B., "Cast coils for electrical machines and their application in automotive and industrial drive systems," 2014. In Electric Drives Production Conference (EDPC), 2014 4th International, S. 17. 
[17] Groninger, M., Horch, F., Kock, A., Pleteit, H., Ponick, B., Schmidt, D. und Wostmann, F.-J., "Casting production of coils for electrical machines," 2011. In 2011 1st International Electric Drives Production Conference (EDPC), S. 159-161.

[18] Hering, E., Elektrotechnik und Elektronik für Maschinenbauer, 2. Aufl. 2012. VDI-Buch. Berlin, Heidelberg: Springer Berlin Heidelberg, 2012.

[19] Hofmann, P., Hybridfahrzeuge: [ein alternatives Antriebskonzept für die Zukunft]. Wien: Springer, 2010.

[20] Islam, M. J. und Arkkio, A., "Time-stepping finite-element analysis of eddy currents in the form-wound stator winding of a cage induction motor supplied from a sinusoidal voltage source," Electric Power Applications, IET, vol. 2, Nr. 4, S. 256-265, 2008. http://ieeexplore.ieee.org/stamp/stamp.jsp?arnumber $=4555178$.

[21] Islam, M. J. und Arkkio, A., "Effects of pulse-width-modulated supply voltage on eddy currents in the form-wound stator winding of a cage induction motor," Electric Power Applications, IET, vol. 3, Nr. 1, S. 50-58, 2009.

http://ieeexplore.ieee.org/stamp/stamp.jsp?arnumber $=4745917$.

[22] Islam, M. J., Pippuri, J., Perho, J. und Arkkio, A., "Time-harmonic finite-element analysis of eddy currents in the form-wound stator winding of a cage induction motor," Electric Power Applications, IET, vol. 1, Nr. 5, S. 839-846, 2007. http://ieeexplore.ieee.org/stamp/stamp.jsp?arnumber=4295956.

[23] Junginger, C. und Stöhr, G., "Assembling, integration and measurement of a hybrid machine with cast coils," 2014. In 13th International CTI Symposium Automotive Transmissions, HEV and EV Drives, S. 437ff. Düsseldorf: CTI.

[24] Junginger, C. und Stöhr, G., "Design, integration and measurement of a hybrid machine with cast coils," 2014. In Electric Drives Production Conference (EDPC), 2014 4th International, S. 262.

[25] Kamiya, M., "Development of Traction Drive Motors for the Toyota Hybrid System," 2005. In 2005 International Power Electronics Conference.

[26] Kiencke, U. und Eger, R., Messtechnik Systemtheorie für Elektrotechniker, 6th ed. Springer-Lehrbuch. Berlin [u.a.]: Springer, 2005.

[27] Köhring, P., Beitrag zur Berechnung der Stromverdrängung in Niederspannungsasynchronmaschinen mit Kurzschlussläufern mittlerer bis großer Leistung, TU Bergakademie, Diss--Freiberg, 2009, 2009.

[28] Küpfmüller, K., Theoretische Elektrotechnik: Eine Einführung, [Online-Ausg. der] 18. [gedr.] Aufl. Springer-Lehrbuch. Berlin, Heidelberg: Springer, 2008.

[29] Laible, T., "Stromverdrängung in Nutenleitern von trapezförmigem und dreieckigem Querschnitt," Archiv für Elektrotechnik, S. 558-566, 1933.

[30] Lehner, G., Elektromagnetische Feldtheorie: Für Ingenieure und Physiker. Berlin, Heidelberg: Springer-Verlag Berlin Heidelberg, 2010.

[31] Mocanu, C. I., "Nichtstationäre Stromverdrängung in einer Maschinennut von rechteckigem Querschnitt," Archiv für Elektrotechnik, Nr. 3, S. 164-170, 1973.

[32] Mosebach, H., "Stromverdrängungsprobleme bei PPSM," Jahresberichte. Braunschweig, 2005. https://www.tu-braunschweig.de/imab/institut/jahresberichte/2005. 
[33] Müller, G. und Ponick, B., Grundlagen elektrischer Maschinen, 10., völlig neu bearb. Aufl. Elektrische Maschinen Bd. 1. Weinheim: Wiley-VCH-Verl., 2014.

[34] Müller, G., Vogt, K. und Ponick, B., Berechnung elektrischer Maschinen, 6., völlig neu bearb. Aufl. Elektrische Maschinen Bd. 2. Weinheim: Wiley-VCH-Verl., 2008.

[35] Oberretl, K., "Die Ermittlung von magnetischen Feldern, Wirbelströmen und Kräften in komplizierten Fällen durch Simulation an Gittermodellen," Archiv für Elektrotechnik, Nr. 5, S. 297-313, 1963.

[36] Oberretl, K., "13 Regeln für minimale Zusatzverluste in Induktionsmotoren," Bulletin Oerlikon, Nr. 389/390, S. 1-11, 1969.

[37] Oberretl, K., "Magnetic Fields, Eddy Currents, and Losses, Taking the Variable Permeability into Account," Power Apparatus and Systems, IEEE Transactions on, Nr. 11, S. 1646-1657, 1969.

[38] Oberretl, K., "Zusätzliche Wirbelstromverluste in Nutenleitern infolge eindringendem Luftspaltfeld," Archiv für Elektrotechnik, S. 121-127, 1978.

[39] Park, R. H., "Two Reaction Theory of Synchronous Machines," American Institute of Electrical Engineers, Transactions of the, vol. 48, S. 716-730, 1929.

[40] Paul, S. und Paul, R., Grundlagen der Elektrotechnik und Elektronik 2: Elektromagnetische Felder und ihre Anwendungen. Berlin, Heidelberg: Springer, 2012.

[41] Pesch, B., Bestimmung der Messunsicherheit nach GUM: Grundlagen der Metrologie ; Messunsicherheitseinflüsse, Messunsicherheitsanalyse und -budgets, Verteilungen, Sensitivitätskoeffizienten und Gewichtungsfaktoren, Korrelation, Ergebnisse darstellen, Optimierungspotentiale, Beispiele, ausführliches Glossar. Alamogordo NM USA: Pesch, 2004.

[42] Petermaier, K., "Simulation einer Drehfeldmaschine mit Sinus-Dreieckmodulierten Pulsmustern," 2014. In ANSYS Conference \& 32. CADFEM Users Meeting, 2014.

[43] Reichert, K., "Über einseitige Stromverdrängung in reckteckförmigen Hohlleitern," Archiv für Elektrotechnik, Nr. 1, S. 58-74, 1966.

[44] Richter, J., Dollinger, A. und Doppelbauer, M., "Iron loss and parameter measurement of permanent magnet synchronous machines," 2014. In International Conference on Electrical Machines, 1635-41.

http://ieeexplore.ieee.org/stamp/stamp.jsp?arnumber $=6960401$.

[45] Richter, R., "Über zusätzliche Kupferverluste," Archiv für Elektrotechnik, Nr. 12, S. 518-526, 1914.

[46] Richter, R., "Über zusätzliche Stromwärme: II. Entwurf von Nutenwicklungen," Archiv für Elektrotechnik, Nr. 1 und 2, S. 1-35, 1915.

[47] Richter, R., "Über zusätzliche Stromwärme: I. Einfluss der Leitfähigkeit. Abnahme des Wechselstromwiderstandes mit zunehmender Temperatur.," Archiv für Elektrotechnik, Nr. 7, S. 175-187, 1915.

[48] Richter, R., "Über zusätzliche Stromwärme: III. Nutenwicklungen mit unterteilten Leitern," Archiv für Elektrotechnik, Nr. 1 und 2, S. 1-52, 1916. 
[49] Richter, R., "Über zusätzliche Stromwärme: IV. Unterdrückung der zusätzlichen Stromwärme durch magnetische Hilfskreise," Archiv für Elektrotechnik, Nr. 11, S. 335-375, 1917.

[50] Richter, R., Elektrische Maschinen: Erster Band: Allgemeine Berechnungselemente, Die Gleichstrommaschinen. Basel [u.a.]: Birkhäuser, 1967.

[51] Rogowski, W., "Über zusätzliche Kupferverluste, über die kritische Kupferhöhe einer Nut und über das kritische Widerstandsverhältnis einer Wechselstrommaschine," Archiv für Elektrotechnik, Nr. 3, S. 81-118, 1913.

[52] Rogowski, W., "Über zusätzliche Kupferverluste. Einige Bemerkungen zu dem gleichnamigen Aufsatze des Herrn Richter.," Archiv für Elektrotechnik, Nr. 12, S. 526-529, 1914.

[53] Schröder, D., Elektrische Antriebe - Regelung von Antriebssystemen, 3rd ed. Berlin Heidelberg: Springer Berlin Heidelberg, 2009.

[54] Schunk, H., Stromverdrängung Strom- und Feldverdrängung in elektrischen Leitern ; mit 19 Tabellen. Uni-Taschenbücher 379 Fachbuchreihe angewandte Elektronik für Fachhochschulen. Heidelberg: Hüthig, 1975.

[55] Sommerfeld, A., "Über das Wechselfeld und den Wechselstromwiderstand von Spulen und Rollen," Annalen der Physik, Nr. Band 15, S. 673-708, 1904.

[56] Stoll, R. L., The analysis of eddy currents. Stoll, Richard L. Oxford: Clarendon Press, 1974.

[57] Yilmaz, M. und Krein, P. T., "Capabilities of finite element analysis and magnetic equivalent circuits for electrical machine analysis and design," 2008. In Power Electronics Specialists Conference, 2008. PESC 2008. IEEE, 4027-33. 


\section{Anhang}

\section{Anhang A: Widerstandsbestimmung gegossener Spulen}

Die Widerstandsbestimmung der gegossenen Spulen wurde zum einen anhand einer Messreihe bestimmt. Dabei wurden 25 Spulen ausgewertet:

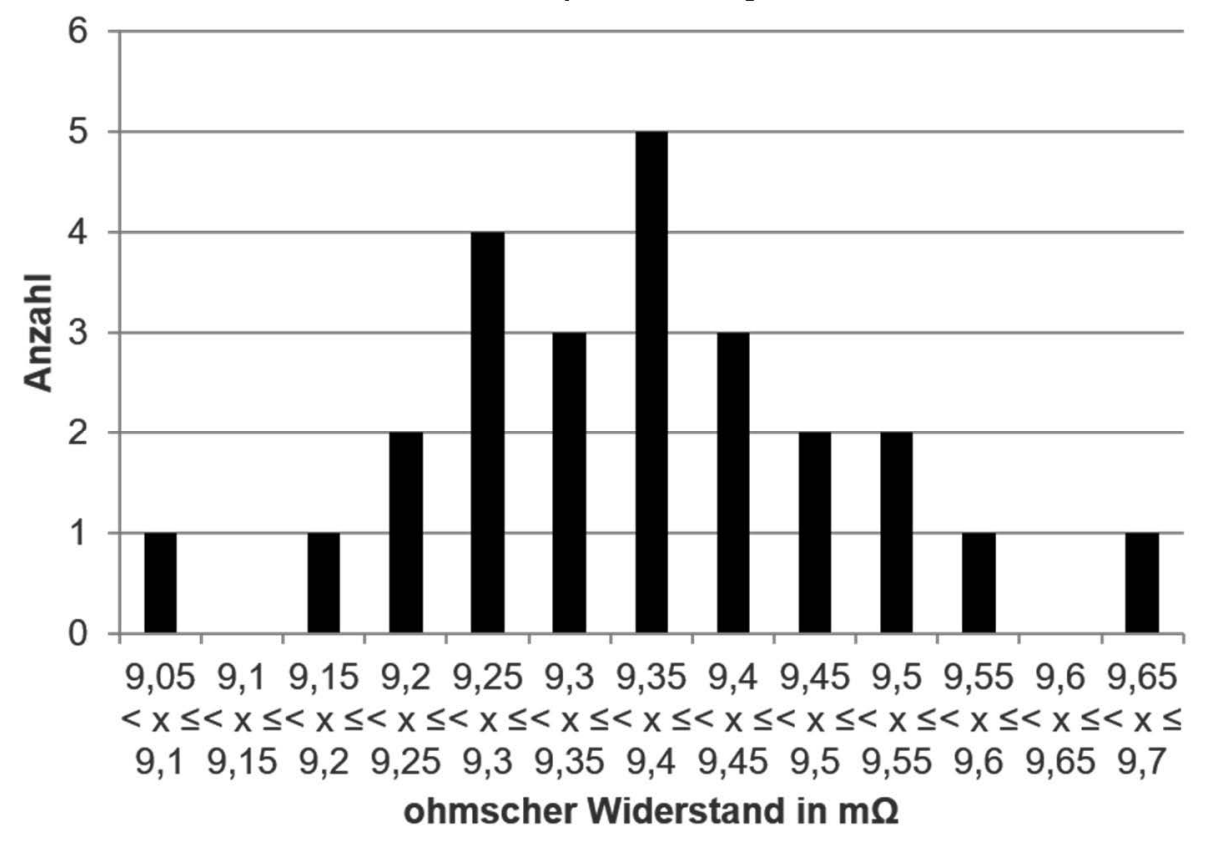

Abbildung A-1: Auswertung der Messreihe zur Bestimmung des elektrischen Widerstands

Es wurde ein mittlerer ohmscher Widerstand von 9,38 $\mathrm{m} \Omega$ mit einer Standardabweichung von 0,13 ermittelt.

Des Weiteren wurde der Widerstand der gegossenen Spulen analytisch gemäß Gleichung (2-2) ermittelt. Dies führt auf:

\begin{tabular}{|c|c|}
\hline Parameter & Werte \\
\hline Mittlerer Leiterquerschnitt in $\mathbf{~ m m}^{2}$ & 6,88 \\
\hline$\rho$ in $\Omega \mathrm{mm}^{2} / \mathrm{m}$ & 0,0176 \\
\hline Länge der Nut in mm & 95 \\
\hline Länge im Wickelkopf in mm & 35,85 \\
\hline Länge einer Windung in mm & 261,7 \\
\hline Anzahl der Windungen & 14 \\
\hline Leiterlänge einer Spule in mm & 3663,8 \\
\hline Widerstand in $\mathbf{m} \Omega$ & 9,37 \\
\hline
\end{tabular}

Tabelle A-1: Für analytische Bestimmung des Widerstands verwendete Eigenschaften 


\section{Anhang B: Messunsicherheit der $k_{r}$-Faktor-Bestimmung}

Die durchgeführte Messunsicherheitsanalyse basiert auf Pesch [41], Kiencke u.a. [26] und Böhme [4].

Die Messunsicherheitsanalyse wird auf Grundlage der Budgetgleichung der Messunsicherheit

$$
U_{k}=k \sqrt{\sum_{i=1}^{n} G_{i}\left(c_{i} u_{i}\right)^{2}}
$$

durchgeführt. Mit dieser wird die Unsicherheit des besten Schätzwerts $U_{k}$ ermittelt. Die Budgetgleichung geht auf die Gauß'sche Fehlerfortpflanzung zurück. Sie gibt das Intervall an, in dem der Messwert mit einer bestimmten Wahrscheinlichkeit liegt.

Dazu müssen zunächst die Einflussgrößen $u_{i}$, die auf das ideale Messmodell einwirken, auf Basis eigener oder fremder Erkenntnisse abgeschätzt werden. Dabei wird sich auf die wesentlichen Faktoren beschränkt. Im nächsten Schritt werden die Sensitivitätskoeffizienten $c_{i}$ ermittelt. Diese geben an wie stark die Gesamtmessunsicherheit von den einzelnen Einflussgrößen abhängt. Des Weiteren wird der Gewichtungsfaktor $G_{i}$ benötigt. Mit diesem werden Messgrößen so normiert, als ob eine Einflussgröße mit Normalverteilung vorlege. Der Gewichtungsfaktor hängt von der Art der Verteilung ab. Als letzte Größe geht der Erweiterungsfaktor aus der tVerteilung $k$ in die Gleichung ein. Er berücksichtigt die Datenmenge, die der Bestimmung der Einflussgrößen zugrunde liegt. Je geringer die Datenmenge ist, desto unsicherer ist die Annahme, dass die Verteilung dieser Reihe auf einer Normalverteilung basiert. Der t-Faktor gibt den Faktor an, mit dem eine Größe multipliziert werden muss, um die gleiche Überdeckungswahrscheinlichkeit wie für eine Normalverteilung zu erhalten. Der t-Faktor kann der Tabelle A-2 für verschiedene Freiheitsgrade entnommen werden.

Im Folgenden werden die Unsicherheiten der besten Schätzwerte für die verschiedenen Frequenzen, bei denen die E-Maschine am Prüfstand betrieben wird, ermittelt. Für die Einflüsse am Prüfstand $P_{\text {ges }}$ - Prüfstand liegen Kalibrierscheine der einzelnen Hersteller vor, aus denen der angenommene Unsicherheitsbeitrag bestimmt werden kann. Für die Eisenverluste $P_{F e}$ wird auf Basis der Vorversuche aus Anhang D und der 2D-FEM-Berechnungen eine Abschätzung vorgenommen. In den Eisenverlusten sind die lastunabhängigen Verluste durch Nutungsoberwellen und die lastabhängigen Verluste durch Wicklungsoberwellen enthalten. Diese zusätzliche Einflussgröße erhöht den Messunsicherheitsbeitrag. Auch die Reibverluste $P_{\text {reib }}$ werden mit Hilfe des Vorversuchs aus Anhang $D$ ermittelt. Hier kann ebenfalls auf die Kalibrierscheine der Hersteller zurückgegriffen werden. Die Magnetverluste $P_{\text {mag }}$ werden mit Hilfe der 3DFEM-Berechnung ermittelt. Der Umrichterbetrieb der E-Maschine stellt einen Messunsicherheitsbeitrag dar, der sich auf die Eisen-, die Magnet- und die Kupferverluste auswirkt. Er verursacht zusätzliche Verluste, die in der FEM-Simulation und in der analytischen Berechnung nicht berücksichtigt werden, da dort mit sinusförmigen Spannungen gerechnet wird. Wie aus Petermaiers [42] Untersuchungen hervorgeht, spielt hierbei der Modulationsgrad eine entscheidende Rolle. Anhand seiner Ergebnis- 
se ist zu erkennen, dass eine Zwischenkreisspannung nahe an der Strangspannung von Vorteil ist, um möglichst geringe zusätzliche Verluste zu verursachen. Diese Erkenntnisse erlauben es im Vorfeld, die zusätzlichen Verluste zu reduzieren. Jedoch liegt hierin ein wesentlicher Einflussfaktor, der einen zusätzlichen Unsicherheitsbeitrag für alle Verlustarten bedeutet. Die Durchführung der Messunsicherheitsanalyse wird im Folgenden sortiert nach der Betriebsfrequenz durchgeführt:

\begin{tabular}{|c|c|c|c|c|c|c|c|}
\hline $107 \mathrm{~Hz}$ & 0.009 & 0.15 & 0.1 & 0.3 & & & \\
\hline Einflussgröße & $\mathrm{P}_{\text {ges }}$ - Prüfstand & $\mathrm{P}_{\mathrm{Fe}}$ & Preib & $P_{\text {mag }}$ & $\mathrm{P}_{\mathrm{cu}}$ & & Gleichstromverluste \\
\hline Schätzwert & 3342.594083 & 178.4884007 & 22 & 25.36472691 & 3174.980874 & & 2445.964063 \\
\hline Angenommener Unsicherheitsbeitrag & 30.08334674 & 26.77326011 & 2.2 & 7.609418074 & & & \\
\hline Verteilung & $\mathrm{N}$ & $\mathrm{N}$ & $\mathrm{N}$ & $\mathrm{R}$ & & & \\
\hline Gewichtung & 1 & 1 & 1 & 0.577350269 & & & \\
\hline Standardmessunsicherheit & 30.08334674 & 26.77326011 & 2.2 & 4.393299573 & & & \\
\hline Freiheitgrad & unendlich & 10 & 10 & unendlich & Gesamtunsicherheit & & \\
\hline \multirow[t]{2}{*}{ Unsicherheitsbeitrag } & 30.08334674 & 26.77326011 & 2.2 & 2.536472691 & 40.41149468 & & \\
\hline & & & & & 83.65179398 & 0.02634718 & \\
\hline & & & & & & & \\
\hline$v$ & 51.90328266 & & & & kr & Delta & \\
\hline Freiheitsgrade & 36 & & & plus Stabw. & 1.332248792 & 0.034199928 & \\
\hline \multirow[t]{2}{*}{$\mathrm{k}$ aus Tabelle } & 2.07 & & & Mittelwert & 1.298048864 & & \\
\hline & & & & minus Stabw. & 1.263848936 & & \\
\hline
\end{tabular}

\begin{tabular}{|c|c|c|c|c|c|c|c|}
\hline $200 \mathrm{~Hz}$ & 0.005 & 0.15 & 0.1 & 0.3 & & & \\
\hline Einflussgröße & $P_{\text {ges }}$ - Prüfstand & $\mathrm{P}_{\mathrm{Fe}}$ & $P_{\text {reib }}$ & $P_{\text {mag }}$ & $\mathrm{P}_{\mathrm{cu}}$ & & Gleichstromverluste \\
\hline Schätzwert & 4217.666865 & 688.7383796 & 50 & 66.06023293 & 3643.978748 & & 2446.195743 \\
\hline Angenommener Unsicherheitsbeitrag & 21.08833432 & 103.3107569 & 5 & 19.81806988 & & & \\
\hline Verteilung & $\mathrm{N}$ & $\mathrm{N}$ & $\mathrm{N}$ & $R$ & & & \\
\hline Gewichtung & 1 & 1 & 1 & 0.577350269 & & & \\
\hline Standardmessunsicherheit & 21.08833432 & 103.3107569 & 5 & 11.44196798 & & & \\
\hline Freiheitgrad & unendlich & 10 & 10 & unendlich & Gesamtunsicherheit & & \\
\hline \multirow[t]{4}{*}{ Unsicherheitsbeitrag } & 21.08833432 & 103.3107569 & 5 & 6.606023293 & 105.7661094 & & \\
\hline & & & & & 235.8584239 & 0.064725521 & \\
\hline & & & & & & & \\
\hline & & & & & & & \\
\hline $\mathrm{v}$ & 10.98503788 & & & & $\mathbf{k r}$ & Delta & \\
\hline Freiheitsgrade & 10 & & & plus Stabw. & 1.586069791 & 0.096418459 & \\
\hline \multirow[t]{2}{*}{$\mathrm{k}$ aus Tabelle } & 2.23 & & & Mittelwert & 1.489651332 & & \\
\hline & & & & minus Stabw. & 1.393232873 & & \\
\hline
\end{tabular}

\begin{tabular}{|c|c|c|c|c|c|c|c|}
\hline $267 \mathrm{~Hz}$ & 0.004 & 0.15 & 0.1 & 0.3 & & & \\
\hline Einflussgröße & $P_{\text {ges }}$ - Prüfstand & $\mathrm{P}_{\mathrm{Fe}}$ & $P_{\text {reib }}$ & $P_{\text {mag }}$ & $\mathrm{P}_{\mathrm{cu}}$ & & Gleichstromverluste \\
\hline Schätzwert & 5062.64166 & 1037.222465 & 74 & 104.9618605 & 3978.797986 & & 2429.196479 \\
\hline Angenommener Unsicherheitsbeitrag & 20.25056664 & 155.5833697 & 7.4 & 31.48855815 & & & \\
\hline Verteilung & $\mathrm{N}$ & $\mathrm{N}$ & $\mathrm{N}$ & $\mathrm{R}$ & & & \\
\hline Gewichtung & 1 & 1 & 1 & 0.577350269 & & & \\
\hline Standardmessunsicherheit & 20.25056664 & 155.5833697 & 7.4 & 18.17992752 & & & \\
\hline Freiheitgrad & unendlich & 10 & 10 & unendlich & Gesamtunsicherheit & & \\
\hline \multirow[t]{4}{*}{ Unsicherheitsbeitrag } & 20.25056664 & 155.5833697 & 7.4 & 10.49618605 & 157.4204571 & & \\
\hline & & & & & 355.770233 & 0.089416511 & \\
\hline & & & & & & & \\
\hline & & & & & & & \\
\hline $\mathrm{v}$ & 10.4806872 & & & & $\mathbf{k r}$ & Delta & \\
\hline Freiheitsgrade & 9 & & & plus Stabw. & 1.784362959 & 0.146455931 & \\
\hline \multirow[t]{2}{*}{$\mathrm{k}$ aus Tabelle } & 2.26 & & & Mittelwert & 1.637907028 & & \\
\hline & & & & minus Stabw. & 1.491451097 & & \\
\hline
\end{tabular}




\begin{tabular}{|c|c|c|c|c|c|c|c|}
\hline $309 \mathrm{~Hz}$ & 0.003 & 0.15 & 0.1 & 0.3 & & & \\
\hline Einflussgröße & $P_{\text {ges }}$ - Prüfstand & $\mathrm{P}_{\mathrm{Fe}}$ & $P_{\text {reib }}$ & $P_{\text {mag }}$ & $\mathrm{P}_{\mathrm{cu}}$ & & Gleichstromverluste \\
\hline Schätzwert & 5662.248934 & 1287.209895 & 125 & 133.1169525 & 4156.842684 & & 2408.691676 \\
\hline Angenommener Unsicherheitsbeitrag & 16.9867468 & \begin{tabular}{|l|}
193.0814842 \\
\end{tabular} & 12.5 & 39.93508576 & & & \\
\hline Verteilung & $\mathrm{N}$ & $\mathrm{N}$ & $\mathrm{N}$ & $\mathrm{R}$ & & & \\
\hline Gewichtung & 1 & 1 & 1 & 0.577350269 & & & \\
\hline Standardmessunsicherheit & 16.9867468 & 193.0814842 & 12.5 & 23.05653251 & & & \\
\hline Freiheitgrad & unendlich & 10 & 10 & unendlich & Gesamtunsicherheit & & \\
\hline \multirow[t]{2}{*}{ Unsicherheitsbeitrag } & 16.9867468 & 193.0814842 & 12.5 & 13.31169525 & 194.6855422 & & \\
\hline & & & & & 439.9893254 & 0.105846999 & \\
\hline & & & & & & & \\
\hline $\mathrm{v}$ & 10.33628941 & & & & $\mathrm{kr}$ & Delta & \\
\hline Freiheitsgrade & 9 & & & plus Stabw. & 1.908435212 & \begin{tabular}{|l|}
0.18266735 \\
\end{tabular} & \\
\hline \multirow[t]{2}{*}{$\mathrm{k}$ aus Tabelle } & 2.26 & & & Mittelwert & 1.725767862 & & \\
\hline & & & & minus Stabw. & 1.543100512 & & \\
\hline
\end{tabular}

\begin{tabular}{|c|c|c|c|c|c|c|c|}
\hline $400 \mathrm{~Hz}$ & 0.003 & 0.15 & 0.1 & 0.3 & & & \\
\hline Einflussgröße & $P_{\text {ges }}-$ Prüfstand & $\mathrm{P}_{\mathrm{Fe}_{\mathrm{P}}}$ & $P_{\text {reib }}$ & $P_{\text {mag }}$ & $P_{c u}$ & & Gleichstromverluste \\
\hline Schätzwert & 6804.895639 & 1862.849697 & 175 & 204.6697643 & 4401.060989 & & 2368.398713 \\
\hline Angenommener Unsicherheitsbeitrag & 20.41468692 & 279.4274546 & 17.5 & 61.40092928 & & & \\
\hline Verteilung & $\mathrm{N}$ & $\mathrm{N}$ & $\mathrm{N}$ & $\mathrm{R}$ & & & \\
\hline Gewichtung & 1 & 1 & 1 & 0.577350269 & & & \\
\hline Standardmessunsicherheit & 20.41468692 & 279.4274546 & 17.5 & 35.44984305 & & & \\
\hline Freiheitgrad & unendlich & 10 & 10 & unendlich & Gesamtunsicherheit & & \\
\hline \multirow[t]{3}{*}{ Unsicherheitsbeitrag } & 20.41468692 & 279.4274546 & 17.5 & 20.46697643 & 281.463335 & & \\
\hline & & & & & 636.107137 & 0.144534952 & \\
\hline & & & & & & & \\
\hline $\mathrm{v}$ & 10.29447817 & & & & $\mathbf{k r}$ & Delta & \\
\hline Freiheitsgrade & 9 & & & plus Stabw. & 2.12682438 & 0.268581102 & \\
\hline \multirow[t]{2}{*}{$\mathrm{k}$ aus Tabelle } & 2.26 & & & Mittelwert & 1.858243278 & & \\
\hline & & & & minus Stabw. & 1.589662176 & & \\
\hline
\end{tabular}

\begin{tabular}{|c|c|c|c|c|c|c|c|}
\hline $467 \mathrm{~Hz}$ & 0.003 & 0.15 & 0.1 & 0.3 & & & \\
\hline Einflussgröße & $P_{\text {ges }}-$ Prüfstand & $P_{\mathrm{Fe}}$ & $P_{\text {reib }}$ & $P_{\text {mag }}$ & $P_{c u}$ & & Gleichstromverluste \\
\hline Schätzwert & 7226.622853 & 2124.034699 & 275 & 262.2675566 & 4149.997586 & & 2312.311746 \\
\hline Angenommener Unsicherheitsbeitrag & 21.67986856 & 318.6052048 & 27.5 & 78.68026698 & & & \\
\hline Verteilung & $\mathrm{N}$ & $\mathrm{N}$ & $\mathrm{N}$ & $\mathrm{R}$ & & & \\
\hline Gewichtung & 1 & 1 & 1 & 0.577350269 & & & \\
\hline Standardmessunsicherheit & 21.67986856 & 318.6052048 & 27.5 & 45.42607332 & & & \\
\hline Freiheitgrad & unendlich & 10 & 10 & unendlich & Gesamtunsicherheit & & \\
\hline \multirow[t]{4}{*}{ Unsicherheitsbeitrag } & 21.67986856 & 318.6052048 & 27.5 & 26.22675566 & 321.5950652 & & \\
\hline & & & & & 726.8048474 & 0.1751338 & \\
\hline & & & & & & & \\
\hline & 1038010949 & & & & $\mathrm{kr}$ & Delta & \\
\hline Freiheitsgrade & 9 & & & plus Stabw. & 2.109059231 & 0.314319576 & \\
\hline \multirow[t]{2}{*}{$\mathrm{k}$ aus Tabelle } & 2.26 & & & Mittelwert & 1.794739655 & & \\
\hline & & & & minus Stabw. & 1.48042008 & & \\
\hline
\end{tabular}

\begin{tabular}{|c|c|c|c|c|c|c|c|}
\hline $533 \mathrm{~Hz}$ & 0.004 & 0.15 & 0.1 & 0.3 & & & \\
\hline Einflussgröße & $P_{\text {ges }}-$ Prüfstand & $\mathrm{P}_{\mathrm{Fe}}$ & $P_{\text {reib }}$ & $P_{\text {mag }}$ & $P_{\text {cul }}$ & & Gleichstromverluste \\
\hline Schätzwert & 7083.228135 & 2237.462952 & 365 & 284.6943795 & 3603.707895 & & 1976.6568 \\
\hline Angenommener Unsicherheitsbeitrag & 28.33291254 & 335.6194428 & 36.5 & 85.40831384 & & & \\
\hline Verteilung & $\mathrm{N}$ & $\mathrm{N}$ & $\mathrm{N}$ & $\mathrm{R}$ & & & \\
\hline Gewichtung & 1 & 1 & 1 & 0.577350269 & & & \\
\hline Standardmessunsicherheit & 28.33291254 & 335.6194428 & 36.5 & 49.31051298 & & & \\
\hline Freiheitgrad & unendlich & 10 & 10 & unendlich & Gesamtunsicherheit & & \\
\hline \multirow[t]{3}{*}{ Unsicherheitsbeitrag } & 28.33291254 & 335.6194428 & 36.5 & 28.46943795 & 339.9792983 & & \\
\hline & & & & & 768.353214 & 0.213211846 & \\
\hline & & & & & & & \\
\hline $\mathrm{v}$ & 10.52835925 & & & & kr & Delta & \\
\hline Freiheitsgrade & 9 & & & plus Stabw. & 2.211846341 & 0.388713516 & \\
\hline \multirow[t]{2}{*}{$\mathrm{k}$ aus Tabelle } & 2.26 & & & Mittelwert & 1.823132825 & & \\
\hline & & & & minus Stabw. & 1.434419309 & & \\
\hline
\end{tabular}




\begin{tabular}{|c|c|c|c|c|c|c|c|}
\hline $600 \mathrm{~Hz}$ & 0.004 & 0.15 & 0.1 & 0.3 & & & \\
\hline Einflussgröße & $P_{\text {ges }}-$ Prüfstand & $\mathrm{P}_{\mathrm{Fe}}$ & $P_{\text {reib }}$ & $P_{\text {mag }}$ & $P_{c u}$ & & Gleichstromverluste \\
\hline Schätzwert & 7281.995804 & 2426.55723 & 494 & 309.712095 & 3393.556289 & & 1737.662128 \\
\hline Angenommener Unsicherheitsbeitrag & 29.12798322 & 363.9835845 & 49.4 & 92.9136285 & & & \\
\hline Verteilung & $\mathrm{N}$ & $\mathrm{N}$ & $\mathrm{N}$ & $\mathrm{R}$ & & & \\
\hline Gewichtung & 1 & 1 & 1 & 0.577350269 & & & \\
\hline Standardmessunsicherheit & 29.12798322 & 363.9835845 & 49.4 & 53.64370843 & & & \\
\hline Freiheitgrad & unendlich & 10 & 10 & unendlich & Gesamtunsicherheit & & \\
\hline \multirow[t]{4}{*}{ Unsicherheitsbeitrag } & 29.12798322 & 363.9835845 & 49.4 & 30.9712095 & 369.7729911 & & \\
\hline & & & & & 835.68696 & 0.246256991 & \\
\hline & & & & & & & \\
\hline & & & & & & & \\
\hline $\mathrm{v}$ & 10.64795545 & & & & kr & Delta & \\
\hline Freiheitsgrade & 9 & & & plus Stabw. & 2.433869727 & 0.480926037 & \\
\hline \multirow[t]{2}{*}{$\mathrm{k}$ aus Tabelle } & 2.26 & & & Mittelwert & 1.952943691 & & \\
\hline & & & & minus Stabw. & 1.472017654 & & \\
\hline
\end{tabular}

\begin{tabular}{|c|c|c|c|c|c|c|c|}
\hline $667 \mathrm{~Hz}$ & 0.004 & 0.15 & 0.1 & 0.3 & & & \\
\hline Einflussgröße & $P_{\text {ges }}-$ Prüfstand & $\mathrm{P}_{\mathrm{Fe}}$ & $P_{\text {reib }}$ & $P_{\text {mag }}$ & $P_{c u}$ & & Gleichstromverluste \\
\hline Schätzwert & 7627.422253 & 2640.352995 & 623 & 345.830784 & 3321.670372 & & 1600.754634 \\
\hline Angenommener Unsicherheitsbeitrag & 30.50968901 & 396.0529492 & 62.3 & 103.7492352 & & & \\
\hline Verteilung & $\mathrm{N}$ & $\mathrm{N}$ & $\mathrm{N}$ & $\mathrm{R}$ & & & \\
\hline Gewichtung & 1 & 1 & 1 & 0.577350269 & & & \\
\hline Standardmessunsicherheit & 30.50968901 & 396.0529492 & 62.3 & 59.89964887 & & & \\
\hline Freiheitgrad & unendlich & 10 & 10 & unendlich & Gesamtunsicherheit & & \\
\hline \multirow[t]{4}{*}{ Unsicherheitsbeitrag } & 30.50968901 & 396.0529492 & 62.3 & 34.5830784 & 403.5666723 & & \\
\hline & & & & & 912.0606794 & 0.274578925 & \\
\hline & & & & & & & \\
\hline & 1077413336 & & & & $\mathrm{kr}$ & Delta & \\
\hline Freiheitsgrade & 9 & & & plus Stabw. & 2.644834481 & 0.569769195 & \\
\hline \multirow[t]{2}{*}{$\mathrm{k}$ aus Tabelle } & 2.26 & & & Mittelwert & 2.075065286 & & \\
\hline & & & & minus Stabw. & 1.505296091 & & \\
\hline
\end{tabular}

\begin{tabular}{|c|c|c|c|c|c|c|c|}
\hline $733 \mathrm{~Hz}$ & 0.005 & 0.15 & 0.1 & 0.3 & & & \\
\hline Einflussgröße & $P_{\text {ges }}-$ Prüfstand & $\mathrm{P}_{\mathrm{Fe}}$ & $P_{\text {reib }}$ & $P_{\text {mag }}$ & $P_{\text {cul }}$ & & Gleichstromverluste \\
\hline Schätzwert & 7746.467856 & 2758.380999 & 815 & 360.3638165 & 3106.308376 & & 1399.740423 \\
\hline Angenommener Unsicherheitsbeitrag & 38.73233928 & 413.7571498 & 81.5 & 108.109145 & & & \\
\hline Verteilung & $\mathrm{N}$ & $\mathrm{N}$ & $\mathrm{N}$ & $\mathrm{R}$ & & & \\
\hline Gewichtung & 1 & 1 & 1 & 0.577350269 & & & \\
\hline Standardmessunsicherheit & 38.73233928 & 413.7571498 & 81.5 & 62.41684394 & & & \\
\hline Freiheitgrad & unendlich & 10 & 10 & unendlich & Gesamtunsicherheit & & \\
\hline \multirow[t]{4}{*}{ Unsicherheitsbeitrag } & 38.73233928 & 413.7571498 & 81.5 & 36.03638165 & 425.0129927 & & \\
\hline & & & & & 947.7789736 & 0.305114257 & \\
\hline & & & & & & & \\
\hline & 1111663869 & & & & & & \\
\hline$\frac{\mathrm{V}}{\text { Freiheitsgrade }}$ & $\frac{11.11663869}{10}$ & & & plus Stabw. & $\begin{array}{c}\mathrm{kr} \\
2.896313689\end{array}$ & \begin{tabular}{|c|} 
Delta \\
0.677110526 \\
\end{tabular} & \\
\hline \multirow[t]{2}{*}{$\mathrm{k}$ aus Tabelle } & 2.23 & & & Mittelwert & 2.219203164 & & \\
\hline & & & & minus Stabw. & 1.542092638 & & \\
\hline
\end{tabular}

\begin{tabular}{|c|c|c|c|c|c|c|c|}
\hline $800 \mathrm{~Hz}$ & 0.005 & 0.15 & 0.1 & 0.3 & & & \\
\hline Einflussgröße & $P_{g e s}$ - Prüfstand & $\mathrm{P}_{\mathrm{Fe}}$ & $P_{\text {reib }}$ & $P_{\text {mag }}$ & $P_{c u}$ & & Gleichstromverluste \\
\hline Schätzwert & 8087.309301 & 2955.828184 & 950 & 393.3821733 & 3379.802621 & & 1300.612004 \\
\hline Angenommener Unsicherheitsbeitrag & 40.43654651 & 443.3742276 & 95 & 118.014652 & & & \\
\hline Verteilung & $\mathrm{N}$ & $\mathrm{N}$ & $\mathrm{N}$ & $R$ & & & \\
\hline Gewichtung & 1 & 1 & 1 & 0.577350269 & & & \\
\hline Standardmessunsicherheit & 40.43654651 & 443.3742276 & 95 & 68.1357911 & & & \\
\hline Freiheitgrad & unendlich & 10 & 10 & unendlich & Gesamtunsicherheit & & \\
\hline \multirow[t]{4}{*}{ Unsicherheitsbeitrag } & 40.43654651 & 443.3742276 & 95 & 39.33821733 & 456.9336006 & & \\
\hline & & & & & 1018.961929 & 0.301485632 & \\
\hline & & & & & & & \\
\hline & & & & & & & \\
\hline$v$ & 11.25683223 & & & & kr & Delta & \\
\hline Freiheitsgrade & 10 & & & plus Stabw. & 3.382072851 & 0.783448043 & \\
\hline \multirow[t]{2}{*}{$\mathrm{k}$ aus Tabelle } & 2.23 & & & Mittelwert & 2.598624808 & & \\
\hline & & & & minus Stabw. & 1.815176765 & & \\
\hline
\end{tabular}




\begin{tabular}{|c|c|c|c|c|c|c|c|}
\hline $867 \mathrm{~Hz}$ & 0.005 & 0.15 & 0.1 & 0.3 & & & \\
\hline Einflussgröße & $P_{\text {ges }}-$ Prüfstand & $\mathrm{P}_{\mathrm{Fe}}$ & $P_{\text {reib }}$ & $P_{\text {mag }}$ & $P_{c u}$ & & Gleichstromverluste \\
\hline Schätzwert & 8438.499433 & 3126.57599 & 1150 & 421.114044 & 3553.015732 & & 1199.519958 \\
\hline Angenommener Unsicherheitsbeitrag & 42.19249716 & 468.9863985 & 115 & 126.3342132 & & & \\
\hline Verteilung & $\mathrm{N}$ & $\mathrm{N}$ & $\mathrm{N}$ & $R$ & & & \\
\hline Gewichtung & 1 & 1 & 1 & 0.577350269 & & & \\
\hline Standardmessunsicherheit & 42.19249716 & 468.9863985 & 115 & 72.939092 & & & \\
\hline Freiheitgrad & unendlich & 10 & 10 & unendlich & Gesamtunsicherheit & & \\
\hline \multirow[t]{4}{*}{ Unsicherheitsbeitrag } & 42.19249716 & 468.9863985 & 115 & 42.1114044 & 486.5458038 & & \\
\hline & & & & & 1084.997143 & 0.305373582 & \\
\hline & & & & & & & \\
\hline & & & & & & & \\
\hline $\mathrm{v}$ & 11.54214803 & & & & kr & Delta & \\
\hline Freiheitsgrade & 10 & & & plus Stabw. & 3.866557486 & 0.904526127 & \\
\hline \multirow[t]{2}{*}{$\mathrm{k}$ aus Tabelle } & 2.23 & & & Mittelwert & 2.962031359 & & \\
\hline & & & & minus Stabw. & 2.057505231 & & \\
\hline
\end{tabular}

\begin{tabular}{|c|c|c|c|c|c|c|c|}
\hline $933 \mathrm{~Hz}$ & 0.005 & 0.15 & 0.1 & 0.3 & & & \\
\hline Einflussgröße & $P_{\text {ges }}$ - Prüfstand & $\mathrm{P}_{\mathrm{Fe}}$ & $P_{\text {reib }}$ & $P_{\text {mag }}$ & $P_{c u}$ & & Gleichstromverluste \\
\hline Schätzwert & 8920.036048 & 3289.16304 & 1500 & 447.7639477 & 3340.400681 & & 1110.308 \\
\hline Angenommener Unsicherheitsbeitrag & 44.60018024 & 493.374456 & 150 & 134.3291843 & & & \\
\hline Verteilung & $\mathrm{N}$ & $\mathrm{N}$ & $\mathrm{N}$ & $\mathrm{R}$ & & & \\
\hline Gewichtung & 1 & 1 & 1 & 0.577350269 & & & \\
\hline Standardmessunsicherheit & 44.60018024 & 493.374456 & 150 & 77.55499072 & & & \\
\hline Freiheitgrad & unendlich & 10 & 10 & unendlich & Gesamtunsicherheit & & \\
\hline \multirow[t]{3}{*}{ Unsicherheitsbeitrag } & 44.60018024 & 493.374456 & 150 & 44.77639477 & 519.5309958 & & \\
\hline & & & & & 1158.554121 & 0.346830884 & \\
\hline & & & & & & & \\
\hline $\mathrm{v}$ & 12.19114183 & & & & kr & Delta & \\
\hline Freiheitsgrade & 11 & & & plus Stabw. & 4.051988098 & 1.043452916 & \\
\hline \multirow[t]{2}{*}{$\mathrm{k}$ aus Tabelle } & 2.23 & & & Mittelwert & 3.008535182 & & \\
\hline & & & & minus Stabw. & 1.965082266 & & \\
\hline
\end{tabular}

\begin{tabular}{|c|c|c|c|c|c|}
\hline \multicolumn{2}{|c|}{$\mathrm{P}$} & $68,30 \%$ & $99,73 \%$ & $95,00 \%$ & $99,00 \%$ \\
\hline \multicolumn{2}{|c|}{$1-\mathrm{P}$} & $31,70 \%$ & $0,27 \%$ & $5,00 \%$ & $1,00 \%$ \\
\hline \multirow{6}{*}{$(1)$} & $(1,84)$ & $(235,78)$ & $(12,71)$ & $(63,66)$ \\
& $(2)$ & $(1,32)$ & $(19,21)$ & $(4,30)$ & $(9,92)$ \\
& 3 & 1,20 & 9,22 & 3,18 & 5,84 \\
& 4 & 1,14 & 6,62 & 2,78 & 4,60 \\
& 5 & 1,11 & 5,51 & 2,57 & 4,03 \\
& 6 & 1,09 & 4,90 & 2,45 & 3,71 \\
& 7 & 1,08 & 4,53 & 2,36 & 3,50 \\
& 7 & 1,07 & 4,28 & 2,31 & 3,36 \\
& 8 & 1,06 & 4,09 & 2,26 & 3,25 \\
& 9 & 1,05 & 3,96 & 2,23 & 3,17 \\
& 10 & 1,03 & 3,42 & 2,09 & 2,85 \\
& 20 & 1,01 & 3,16 & 2,01 & 2,68 \\
& 50 & 1,01 & 3,08 & 1,98 & 2,63 \\
& 100 & 1,00 & 3,00 & 1,96 & 2,58 \\
\hline
\end{tabular}

Tabelle A-2: Tabelle einiger t-Quantile 


\section{Anhang C: Bestimmung der Magnetverluste mittels 3D-FEM}

Der Abgleich anhand einer vereinfachten Geometrie mittels Messaufbau (Abbildung A-3) und Finite-Elemente-Rechnung (Abbildung A-2) ergab eine gute Übereinstimmung zwischen 3D-Simulation und Messergebnissen.

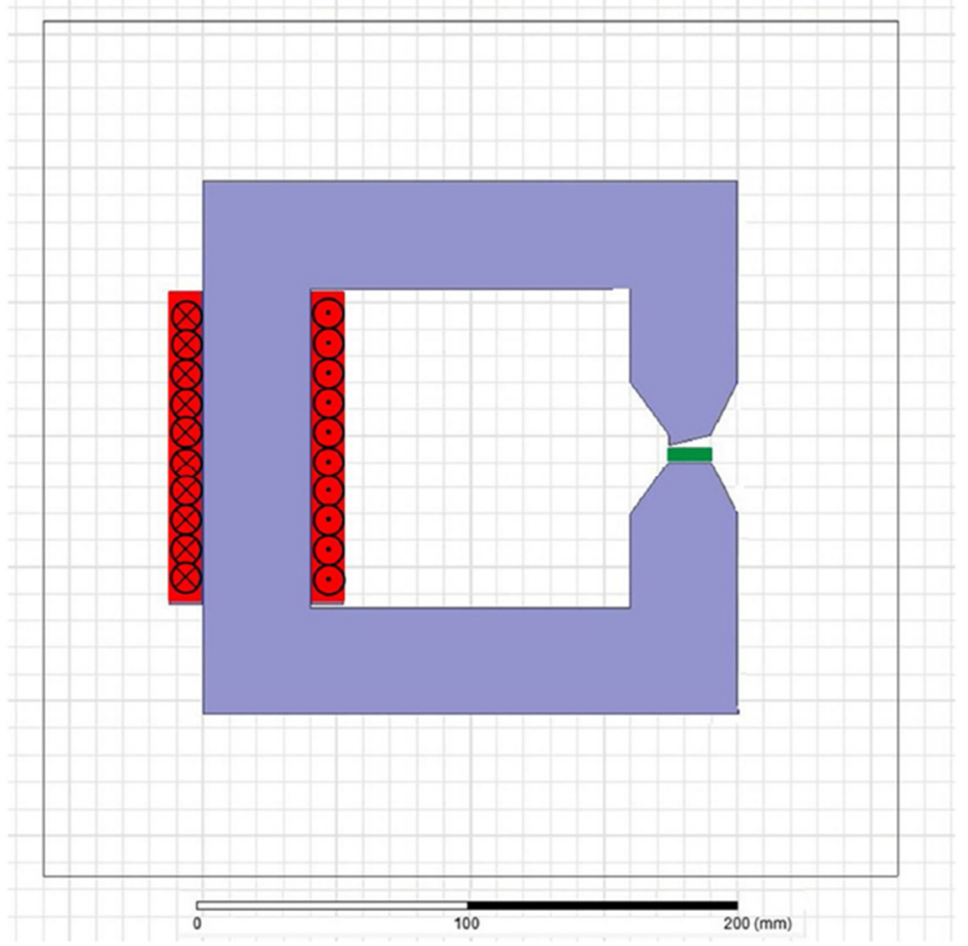

Abbildung A-2: Verwendetes Finite-Elemente-Modell

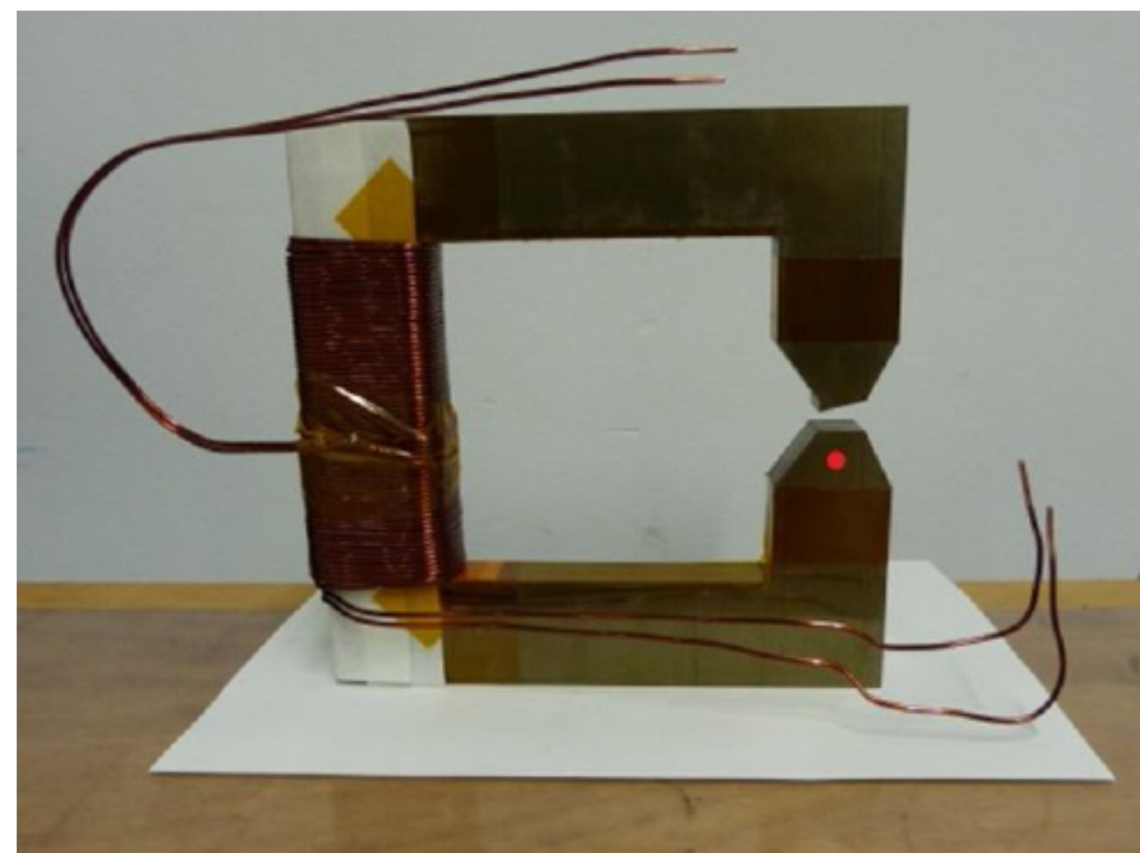

Abbildung A-3: Verwendeter Messaufbau 


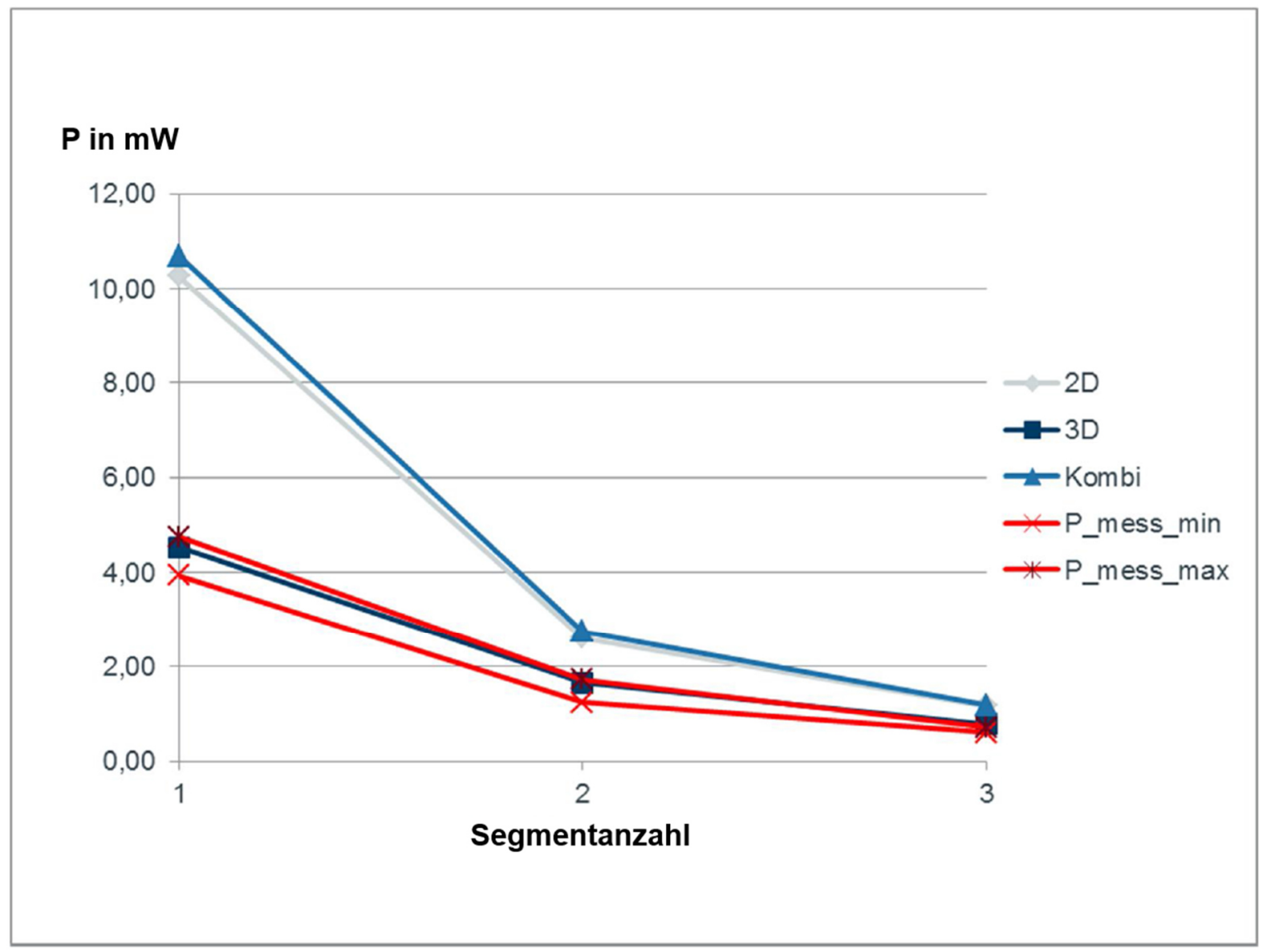

Abbildung A-4: Gegenüberstellung der Verluste in den Magneten auf unterschiedlichen Ermittlungswegen für unterschiedliche Segmentierungen der Magnete

In Abbildung A-4 sind die Ergebnisse der 3D-Simulation, der Messungen, sowie der 2D-Simulation für mehrere Magnetbreiten gegenübergestellt. Es ist zu erkennen, dass die Ergebnisse der 3D-Simulation und der Messungen gut übereinstimmen, während die 2D-Simulation insbesondere bei einem einzelnen breiten Segment deutlich höhere Verluste angibt. 


\section{Anhang D: Ermittlung der Reib- und Eisenverluste}

Für die Bestimmung der Reib- und Eisenverluste werden mehrere Prüfstandsläufe durchgeführt. Dabei wird die Verlustleistung messtechnisch ermittelt. Die Ergebnisse sind in Abbildung A-5 dargestellt.

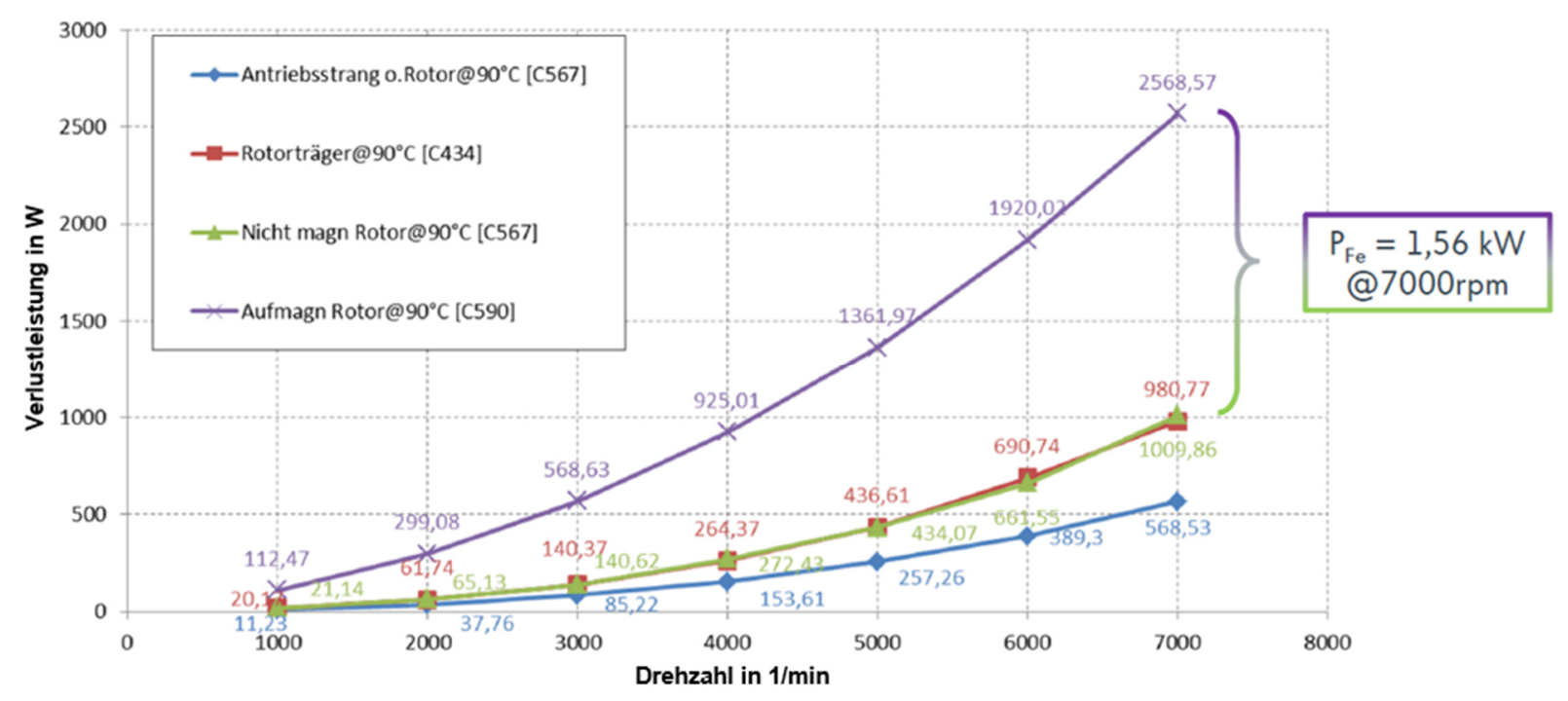

Abbildung A-5: Bestimmung der Reib- und Eisenverluste aus Prüfstandsversuchen

Zunächst erfolgt ein Durchlauf mit nicht magnetisiertem Rotor für die Ermittlung der Reibverluste. Im letzten Prüflauf wird die E-Maschine mit magnetisiertem Rotor im Leerlauf betrieben um über die Differenz zu den Reibverlusten die Eisenverluste im Leerlauf zu erhalten. Diese betragen 1,56 kW bei $7000 \mathrm{U} / \mathrm{min}$. In den so ermittelten Eisenverlusten sind jedoch auch die Magnetverluste $P_{\text {mag }}$ sowie die lastunabhängigen Verluste durch Nutungsoberwellen enthalten. Die Magnetverluste werden mit Hilfe der Erkenntnisse aus Anhang C separiert. Die Verluste durch die Nutungsoberwellen bleiben als Unsicherheit in den Eisenverlusten erhalten. 


\section{Anhang E: Einfluss des Nuthöhe-zu-Nutbreite-Verhältnisses auf das Nutfeld}

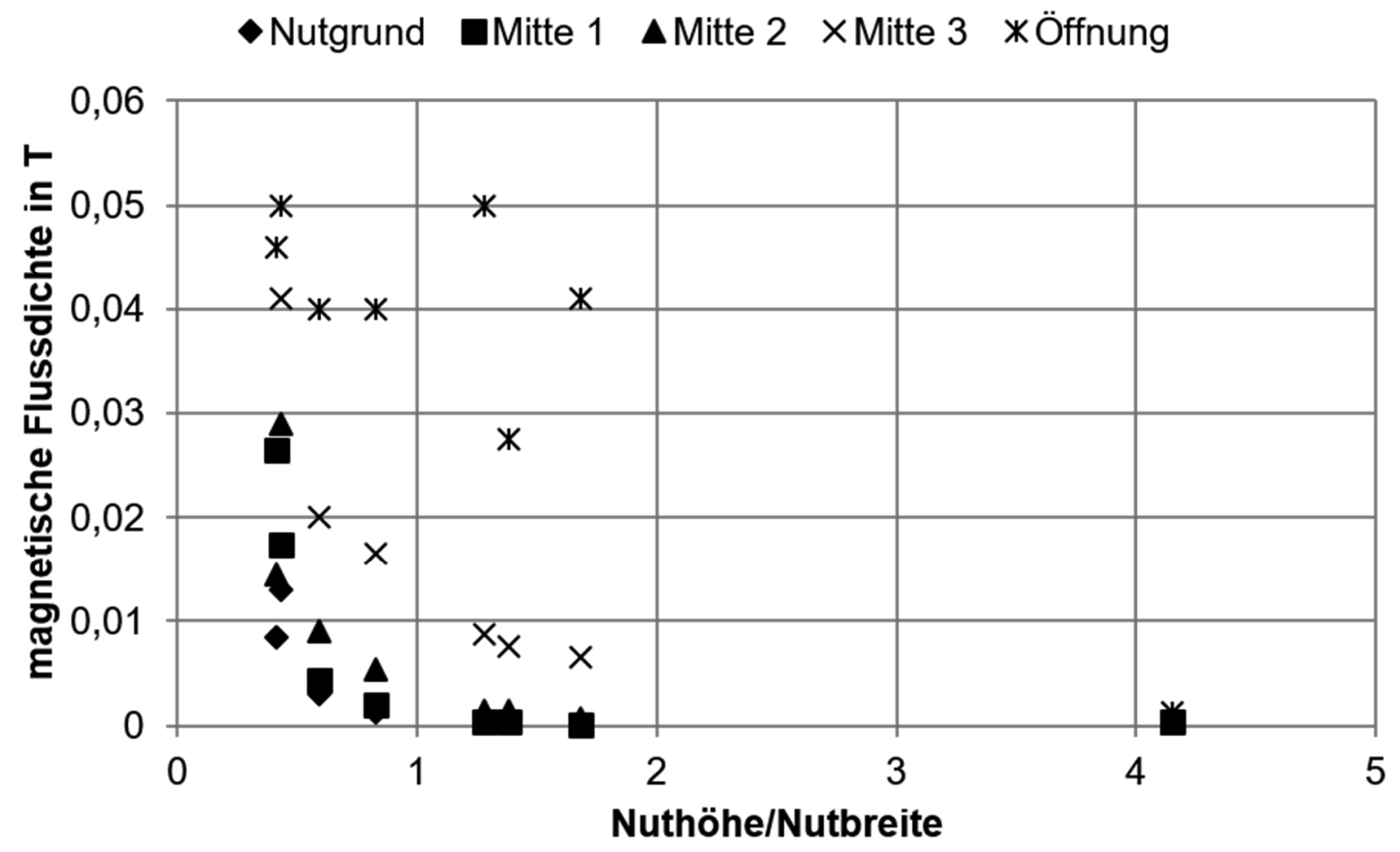

Abbildung A-6: Darstellung der Beeinflussung der magnetischen Flussdichte in der Nut durch das Nuthöhe-zu-Nutbreite-Verhältnis

Aus Abbildung A-6 ist zu erkennen, dass sich die Abhängigkeit der magnetischen Flussdichte vom Nuthöhe-zu-Nutbreite-Verhältnis im unteren Nutbereich (Nutgrund, 1 und 2) stark ähnelt. Für den oberen Nutbereich sind die Verhältnisse nicht so eindeutig. Hier gibt es einen Einfluss durch den Rotor. Dieser beeinflusst den Verlauf der magnetischen Feldlinien im oberen Nutbereich. 